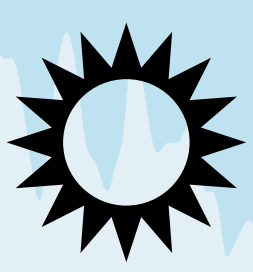

Understanding physical processes and feedback mechanisms between the urban surface and the overlying atmosphere

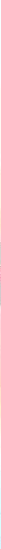

Aristofanis Tsiringakis 



\section{Propositions}

1. Any study of the urban surface energy balance is not complete without considering the effects of boundary-layer turbulence and advection. (this thesis)

2. Momentum advection is an important process for urban weather, which is often ignored in the presence of heat and moisture advection. (this thesis)

3. Unregulated co-operation between business and academia can damage scientific integrity.

4. Effective communication of scientific research requires a full disclosure of the research limitations.

5. Climate change is not the only pressing problem of the $21^{\text {st }}$ century.

6. Maintaining well-informed citizens is one of the greatest challenges of the $21^{\text {st }}$ century.

7. The fundamental moral rules that hold our societies together are alas only a fiction.

Propositions belonging to the thesis, entitled

Understanding physical processes and feedback mechanisms between the urban surface and the overlying atmosphere

Aristofanis Tsiringakis

Wageningen, 21 April 2021 


\section{Understanding physical processes and feedback mechanisms between the urban surface and the overlying atmosphere}




\section{Thesis committee}

\section{Promotor}

Prof. Dr. A.A.M. Holtslag

Emeritus Professor of Meteorology

Wageningen University \& Research

\section{Co-promotor}

Dr. G.J. Steeneveld

Associate Professor, Meteorology and Air Quality group,

Wageningen University \& Research

\section{Other members}

Dr A.J. Teuling, Wageningen University \& Research

Dr A. Hellsten, Finnish Meteorological Institute, Helsinki, Finland

Prof. Dr B.J.H. van de Wiel, Delft University of Technology

Prof. Dr B.J.J.M. van den Hurk, Vrije Universiteit Amsterdam

This research was conducted under the auspices of the Graduate School

for Socio-Economic and Natural Sciences of the Environment (SENSE) 


\title{
Understanding physical processes and feedback mechanisms between the urban surface and the overlying atmosphere
}

\author{
Aristofanis Tsiringakis
}

Thesis

submitted in fulfilment of the requirements for the degree of doctor at

Wageningen University

by the authority of the Rector Magnificus

Prof. Dr A.P.J. Mol,

in the presence of the

Thesis Committee appointed by the Academic Board

to be defended in public

on Wednesday 21 April 2021

at 4 p.m. in the Aula. 
Aristofanis Tsiringakis

Understanding physical processes and feedback mechanisms between the urban surface and the overlying atmosphere

177 pages.

PhD thesis, Wageningen University, Wageningen, the Netherlands (2021)

With references, with summary in English

ISBN 978-94-6395-708-3

DOI https://doi.org/10.18174/541524 


\section{Summary}

This thesis aims at elucidating the effects of urban surface-urban boundary layer-mesoscale flows interactions on the surface energy balance and turbulent exchange in the urban boundary layer (UBL). At first, the role of urban surface-atmosphere interactions on the quantification of the surface energy balance over urban areas in urban canopy models (UCMs) is studied. Then, I investigate how uncertainties in the external atmospheric forcing and surface parameters can affect the surface energy balance and thus the coupling between the urban surface and overlying UBL. Finally, an analysis on the interactions between the urban surface and the low-level jet is studied to investigate their effect on the turbulent exchange in the urban boundary layer and the low-level jet characteristics.

The surface energy balance is different over cities compared to the surrounding rural areas due to differences in heat storage, evapo-transpiration and anthropogenic activities. Consequently, the urban boundary layer varies substantially, in its structure, from its rural counterpart. This has implications for the interactions between urban areas and meso-scale atmospheric circulations. Many previous studies have shown that urban areas affect human thermal comfort and meso-scale flows such as low-level jets, rain and clouds amongst others. Understanding these interactions is essential to improve the representation of urban surface process in urban canopy models (UCMs) and their coupling with numerical weather prediction (NWP) models. This can lead to better weather forecasts in urban areas, with consequences for human health and economic activities in cities.

In Chapter 2 we take essential step towards understanding the interactions between the urban surface and meso-scale flows by investigating the importance of the coupling between the urban surface and the overlying UBL on the surface energy balance and turbulent exchange fluxes. In this chapter we answer the following research question:

Q1. What is the effect of urban surface-atmospheric feedback mechanisms on the surface energy balance over cities?

The skill of a UCM in quantifying the surface energy balance strongly depends on the accurate prescription of many surface parameters, including some that are notoriously difficult to estimate (albedo, thermal conductivity, heat capacities etc.). Therefore, offline optimisation approaches for these parameters are often used, but these optimizations 
exclude the effects of the urban surface-atmosphere feedback mechanisms. Consequently, they can lead to a skewed surface parameter optimisation with consequences for the estimation of the surface energy balance and turbulent exchange fluxes.

To identify the feedback mechanisms and their importance on the surface energy balance two modelling setup are used, the off-line single-layer urban canopy model (SLUCM) and the SLUCM coupled to the single-column version of the Weather Research and Forecasting (WRF) model. The response of the modelled energy balanced to changes in uncertain surface parameters is investigated, during 2 summer days over London. The model responds differently to changes in surface parameter when coupled to the atmosphere. The turbulent heat flux $\left(Q_{H}\right)$ shows up to $22 \%$ lower variability in the on-line setup, because near surface potential temperature gradient and atmospheric stability are altered when albedo changes as any change in heat fluxes directly affects the overlying atmosphere. Consequently, the energy is directed toward the urban fabric changing the variability of the storage heat flux $\left(\Delta Q_{s}\right)$ up to $50 \%$ compared to the off-line model. Moreover, entrainment of heat and moisture affect the near surface humidity altering the latent heat flux $\left(Q_{e}\right)$, an effect that does not occur when the model is in offline mode.

These results highlight the importance of urban surface - boundary layer coupling for an accurate quantification of the surface energy balance. Any change in near surface gradients of potential temperature, moisture and wind strongly impacts the atmospheric stability and the exchange coefficients of heat moisture and momentum. Changes in surface fluxes affect the UBL depth and the entrainment of heat, moisture and momentum in the UBL. The surface fluxes react to the meteorological forcing, but only when the model is coupled to the atmosphere. As such, we conclude that the urban-surface atmosphere coupling is an essential component that should not be omitted when UCMs are developed and evaluated.

In Chapter 2 we investigated the effect of atmospheric coupling on the surface energy balance and turbulent heat fluxes due to changes in surface conditions. However, the effects of the uncertainty in the external atmospheric forcing on the surface energy balance and the coupling between the surface and the overlying atmosphere was not address. Thus, this is investigated in Chapter 3:

Q2. What is the role of uncertainty in the external atmospheric forcing on the urban surface energy balance?

Once a UCM is coupled to the atmosphere, atmospheric forcing provided by other parts of the atmospheric model is needed to compute the surface energy balance. This forcing comes in the form of incoming short- and long-wave radiation and advection of heat moisture and momentum. However, the atmospheric forcing can carry biases in the UCM, thus affecting the surface energy balance, near surface meteorology and the atmospheric 
stability with consequences for vertical mixing and boundary-layer dynamics. Bias in external atmospheric forcing often arises from an incomplete representation of various physical processes in other parts of the model.

In Chapter 3 the same case study as Chapter 2 is utilized to investigate the impact of the uncertainty in atmospheric forcing using a 1D WRF-SLUCM modelling setup. Uncertainties in the advection of potential temperature, aerosol optical depth and exchange coefficients of heat and momentum strongly affect model performance by altering either the radiative input at the surface (aerosols), or the near surface temperature and moisture gradients (advection and exchanges coefficients). The resulting model responses are similar in magnitude to the changes induced by uncertainty in surface parameters. Moreover, a feedback mechanism between high daytime temperature and increased nocturnal radiative cooling was identified. This mechanism is triggered by an increase in nocturnal atmospheric stability that originates from changes in heat advection and the exchange coefficient of heat. These findings highlight the importance of accurately capturing the boundary-layer dynamics and external meso-scale flows (i.e. advection processes) for the quantification of the surface energy balance and turbulent exchange fluxes.

In Chapters 4 and 5 we investigate the interactions between external meso-scale flows and UBL dynamics on the turbulent exchange and vertical mixing within the urban boundary layer. This investigation is focused on the interactions between low-level jets and urban area and the main research question is:

Q3. How does a low-level jet interact with the urban boundary layer?

Low-level jets (LLJs) are local maxima in the wind profile occurring above the nocturnal boundary layer, usually between 100-500m above ground. They are important phenomena affecting vertical mixing, atmospheric stability and horizontal advection in the nocturnal boundary layer. These processes are important for the 'ventilation' of heat and pollutant from urban areas, with consequences for human thermal comfort and street-level air quality. However, LLJ are also affected by turbulent mixing in the nocturnal boundary layer.

In Chapter 4 we investigate the interactions between LLJ and the urban area using a 2-day case study between 14-16 May 2019 over London. Two Doppler Lidars and two numerical weather prediction models re used to identify differences in LLJ speed, height and fall-off between London and Chilbolton, a rural south south west of London. The LLJ over London are elevated by $80 \mathrm{~m}$ compared to LLJ over Chilbolton, due to a combination of increased vertical mixing in the nocturnal UBL and orographic drag by the topography around London. Using a series of idealised experiments we find that urban and orographic effects contribute equally towards the reported differences over London. Moreover, we find that in areas with deeper UBL, the LLJ appears to be elevated and has 
lower speed. Finally, the presence of LLJ increases the shear induced turbulent kinetic energy in the UBL maintaining part of the vertical mixing many hours after the collapse of the daytime UBL.

In Chapter 5 initial onset stages of the LLJ are investigated, to identify the effects of turbulent mixing and momentum advection in the formation of LLJ over urban areas. Two modelling setups are used, the 1D-WRF and the state-of-the-art PALM LES model in an idealised case study inspired by the meteorological situation during 15 May 2019 over London. The reported increase in turbulent mixing, induced by a large urban fraction, delays the onset of the LLJ up to two hours due to a later decoupling of the flow over the city. The height of the LLJ increases (up to 100m) while its speed is reduced (up to 2 $m s^{-1}$ ) with increasing urban fractions. Yet these effects are strongly dependent on the city size. The delay in the onset of the LLJ and its decreased wind speed over and downwind of the urban areas result in strong momentum advection from the already formed LLJ in the surroundings rural areas. The momentum advection contributes substantially to the formation of LLJs over urban areas, especially in the upwind part and its contributions positively scales with the size of the city. 


\section{Contents}

Page

Summary

Contents

ix

Chapter 1 Introduction

Chapter 2 On- and off-line evaluation of the single-layer urban canopy model in London summertime conditions

Chapter 3 Surface and atmospheric driven variability of the single-layer urban canopy model under clear sky conditions over London

Chapter 4 Interactions between the nocturnal low-level jets and the urban boundary layer: a case study over London

Chapter 5 On the role of turbulence and momentum advection in the formation of nocturnal low-level jets over urban areas

Chapter 6 Synthesis

Appendix

References

List of Publications

Education Certificate 



$$
\text { Chapter } 1
$$

Introduction 


\subsection{A very brief history of urban weather and climate research}

Many of the research papers in the field of urban weather and climate start with the rather cliche sentence: "by 2050 more than $66 \%$ of people will be living in urban areas (UN, 2014)". Some papers might even follow the EU-specific guidelines for urban population and claim that more than $85 \%$ of the world's population lives in urban areas as of 2015 (Pesaresi et al., 2016). Or they could be more continent-specific and point out that in Asia $89 \%$ of the population (73\% in Europe) lives is cities. In Greece, the country I come from, nearly 3 out of the 11 million (27\%) inhabitants live in the metropolis of Athens. For a reader these numbers might be impactful considering that we are talking about human beings, but I suspect that for the author they mainly serve as a motivation statement for the importance of the research rather than an actual concern for the weather and challenges people experience in cities. Yet, the urban climate research existed long before the official UN or EU numbers of urban population and will continue to do so long afterwards.

The earliest verified record of a comprehensive urban climate research dates back to 1833 when Luke Howard, who is considered the pioneer of urban climate research, published his "The Climate of London" study. His research on the climate of London, during an observational period of 10-year, offers unique insights on urban climate and is probably one of the earliest climatological studies (Howard, 2012). To put it in context, this study was conducted before the work of the famous Dutch meteorologist Buys Ballot. During the 1930s and 40s research on the impact of urban areas on temperature increased (Büdel \& Wolf, 1933; Arakawa, 1937, i.e), leading to numerous papers published during the 60 s and 70s (Chandler, 1964, 1967; Hutcheon et al., 1967; Mitchell, 1961; Oke \& East, 1971). This research boom lead to the influential paper of Oke (1973), which showcased the relation between the urban heat island intensity and city size. In the same paper, the physical mechanisms behind the urban heat island are explained, namely the difference in the surface energy partitioning between urban and rural areas in the form of anthropogenic heat flux, thermal storage in the urban fabric and less evapo-transpiration. Nearly 50 years later, this analysis of the physical mechanisms behind the urban heat island is still the cornerstone of much of the modern urban climate research.

The field of urban climate research has expanded rapidly since Oke (1973) paper, with studies investigating the interactions between urban areas and weather phenomena at different temporal and spatial scales. Studies in urban boundary layers improved our understanding of how the city interacts with the overlying atmosphere (Bornstein \& Johnson, 1977; Uno et al., 1988) and highlighted differences in wind speed between urban and rural areas (Bornstein, 1975). Furthermore, cities were shown to affect precipitation and clouds (Changnon et al., 1971; Bornstein \& Lin, 2000; Morris \& Simmonds, 2001; Shepherd, 2009) 
and meso-scale flows (i.e. sea-breeze) (Yoshikado, 1992).

\subsection{The urban surface energy balance}

By the start of the $21^{\text {st }}$ century, the differences in the surface energy balance between urban and rural areas had been thoroughly studied and identified (Arnfield, 2003). However, before discussing the differences between the urban and the rural surface energy balance, it is useful to briefly introduce the rural surface energy balance terms.

The general equation for the surface energy balance reads:

$$
Q^{*}=(1-a) S W_{\text {down }}+\epsilon L W_{\text {down }}-\epsilon \sigma\left(T_{\text {skin }}\right)^{4}=Q_{H}+Q_{E}+\Delta Q_{s},
$$

where, $Q^{*}$ is the net radiation at the surface, $a$ is the surface albedo, $S W_{\text {down }}$ is the downward shortwave radiation at the surface, $\epsilon$ is the surface emissivity, $L W_{\text {down }}$ is the downward longwave radiation at the surface, $\epsilon \sigma\left(T_{\text {skin }}\right)^{4}$ is the upwards emitted longwave radiation from the surface with $\sigma$ being the Stefan Boltzmann constant $\left(5.67 * 10^{-8} \mathrm{~W} \mathrm{~m}^{-2} \mathrm{~K}^{-4}\right)$ and $T_{\text {skin }}$ being the surface skin temperature. $Q_{H}$ and $Q_{E}$ are the surface turbulent heat and latent heat fluxes, while $\Delta Q_{s}$ is the storage heat flux. All flux terms in Eq. 1.1 are in $W m^{-2}$.

Over grassland and cropland $Q_{E}$ is usually 2-5 times larger than $Q_{H}$, depending on the water availability and the type of vegetation. The $\Delta Q_{s}$ is usually a small component of the surface energy balance (typically $<10 \%$ of $Q^{*}$ ). Therefore, the surface energy balance over rural areas is governed primarily by the incoming shortwave radiation, the availability of moisture for evapo-transpiration and the leaf area index of the vegetation.

In contrast, urban areas have a very different energy partitioning. Here, impervious surfaces dominate the land-use fraction and therefore $Q_{E}$ is a relatively small component of the surface energy balance (Figure 1.2.1). Part of the $Q_{E}$, in urban areas, originates from direct plant transpiration from the limited urban vegetation. However, the direct evaporative flux from water stored on impervious surfaces, after rainfall events, is a substantial component of the $Q_{E}$ flux in urban areas (Kotthaus \& Grimmond, 2014a). The anthropogenic latent heat flux also contributes to the total $Q_{E}$ in urban areas.

The materials used to construct buildings, roads and other impervious surfaces have usually very high heat capacity and thermal conductivity. The latter allows for a fast transfer of heat from the surface to the deeper layers of the urban fabric (Figure 1.2.1). Consequently, $\Delta Q_{s}$ can range between $17-58 \%$ of the $Q^{*}$ over urban areas(Grimmond \& Oke, 1999), making it a very important component of the surface energy balance. In addition, the heat stored during the day is slowly released back to the atmosphere at night, warming up the urban surface and counteracting the longwave radiative cooling. 


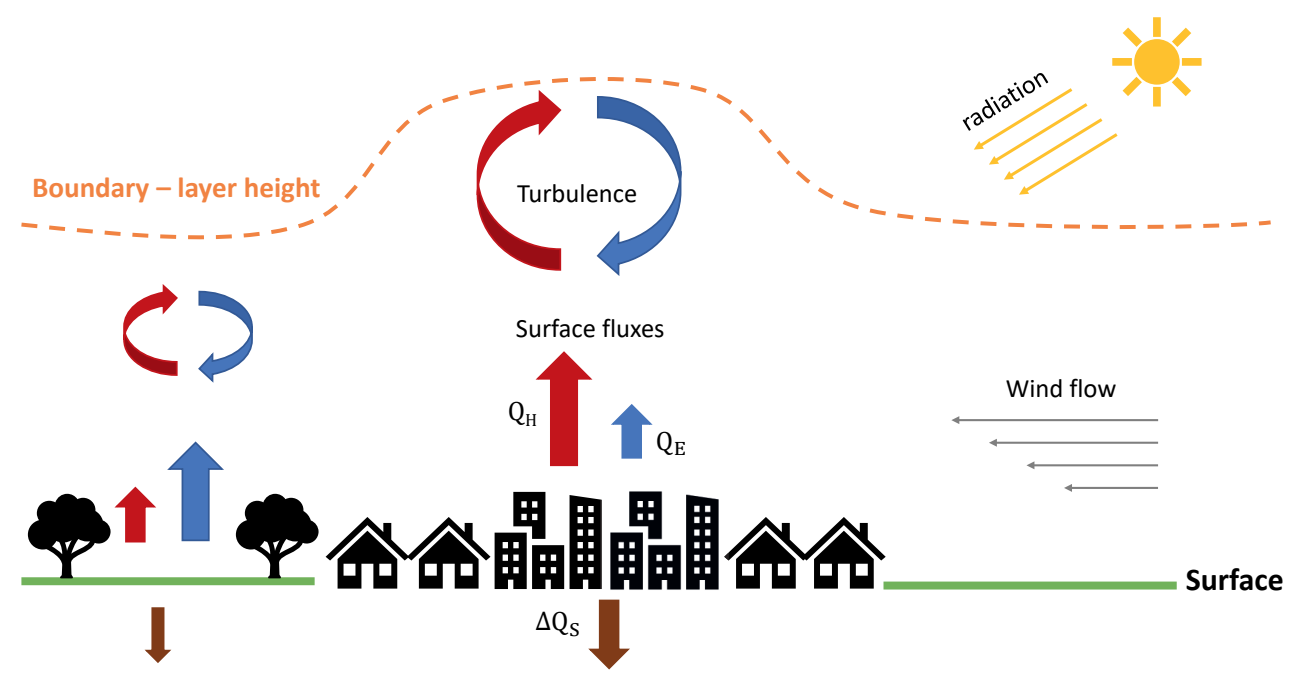

Figure 1.2.1: Conceptual sketch of the differences in surface energy fluxes $\left(Q_{H}, Q_{E}\right.$ and $\left.\Delta Q_{s}\right)$, turbulence and boundary-layer height between urban and rural areas.

The combination of small $Q_{E}$ flux and high surface temperature in urban areas results in a high $Q_{H}$, which warms the air within the urban boundary layer and increases turbulence (Figure 1.2.1). Moreover, the anthropogenic activities (i.e. traffic, equipment usage, heating, air-conditioning) release additional heat near the urban surface, which is known as anthropogenic heat flux $Q_{f}$. $Q_{f}$ can sometimes exceed $Q_{H}$ and is a very important component of the surface energy balance in areas with high population density (i.e. Tokyo, London) with estimated values that can exceed $200 \mathrm{~W} \mathrm{~m}^{-2}$ (Iamarino et al., 2012; Dong et al., 2017).

In addition, the longwave and shortwave radiative fluxes in urban areas can be quite different from their rural surroundings. The generally lower surface albedo and multiple reflections in urban surfaces result in more shortwave radiation being absorbed by the urban fabric. However, large atmospheric concentration of particulate matter (PMs) over urban areas, can decrease the $S W_{\text {down }}$ at the surface and change the partitioning between diffuse and direct radiation (Gomes et al., 2008). Moreover, longwave absorption and emission in the urban canopy can be different due to radiation trapping within urban canyons and differences in the surface emissivity (Best \& Grimmond, 2015).

Therefore, it is necessary to include additional terms in the surface energy balance (Eq. 1.1) for urban areas, which reads (Oke, 1987):

$$
Q^{*}+Q_{f}=Q_{H}+Q_{E}+\Delta Q_{s}+\Delta Q_{a}
$$


where $Q_{f}$ is the anthropogenic heat flux in $W m^{-2}$ and $\Delta Q_{a}$ is the horizontal advective flux in $W m^{-2}$. The latter is not be addressed in this thesis, as it is often deemed negligible in magnitude compared to the remaining components of the energy balance. To summarize, over urban areas the turbulent heat, latent heat and storage fluxes depend on the radiative energy balance at the surface and the supply of heat from anthropogenic activities. Note that although $Q_{f}$ is a separate term in the energy balance equation, it is common practice to add it to the $Q_{H}$, thus combining radiative and anthropogenic effects on the $Q_{H}$. This approach is followed in this thesis as well, so in the following chapters the $Q_{H}$ includes the $Q_{f}$ unless stated otherwise.

\subsection{The urban boundary layer}

The boundary layer is the lowest part of the atmosphere $(1-2 \mathrm{~km})$. It is in direct contact with the surface and responds to changes in surface forcing (i.e. surface energy balance) (Figure 1.2.1). All people, with a few exceptions, live within the boundary layer. Thus our understanding of boundary-layer dynamics and their response to changes in the surface energy balance is essential to forecast weather and air quality. The urban boundary layer (UBL) exhibits distinct differences in its vertical structure compared to its rural counterpart. These differences are a consequence of the differences in the surface energy balance and turbulent transport over urban areas (Figure 1.2.1).

Over a typical homogeneous vegetated surface with minimal roughness elements, the daytime boundary layer consists of three layers: a) the surface layer (also known as inertia sub-layer) occupying the bottom $10 \%$ of the boundary layer, b) the mixed layer $80 \%$ and c) the entrainment zone in the top $10 \%$ of the boundary layer. However, in the presence of various roughness elements (trees, buildings etc.) internal boundary layers (IBLs) may be formed, which alter the structure and turbulent transport in the lower part of the boundary layer (Wieringa, 1993). This is also the case for urban areas, where the various roughness elements (i.e. buildings) create overlapping IBLs and exert substantial drag on the wind flow to form a roughness sub-layer that usually has a depth of 2-5 times the mean building height (Roth, 2000; Barlow, 2014). Above the roughness sub-layer, there is an inertial sub-layer where fluxes are homogeneous and constant with height (Grimmond et al., 2004).

The depth of the urban boundary layer is generally higher compared to a rural one (Pal et al., 2012; Barlow et al., 2015), due to higher $Q_{H}$ and the presence of the urban heat island. Urban boundary layers have a slower rate of decay during the evening transition (Pal et al., 2012; Barlow et al., 2015), due to the presence of an "urban convective island" which maintains part of the turbulence and delays the onset of stable conditions (Barlow et al., 2015). However, during the morning the generally deeper UBL and the large thermal inertia of the urban surface can reduce the growth and heating rates of the 
UBL (Theeuwes et al., 2015). At night, UBLs exhibit more neutral conditions near the surface due to heat release from the urban fabric and the advection of cooler air from the countryside (Uno et al., 1992; Barlow, 2014).

The enhanced surface roughness and more intense vertical mixing can decrease the wind speed in the UBL, especially closer to the surface. However, under certain conditions, the deeper UBL can increase the entrainment of momentum resulting in higher wind speeds over cities (Droste et al., 2018). The enhanced turbulent mixing and delayed collapse of the daytime UBL also affect the formation of low-level jets over urban areas (Wang et al., 2007; Kallistratova \& Kouznetsov, 2012; Barlow et al., 2015).

The different vertical structure and properties of the UBL do not only affect the urban surface but can also influence or even induce meso-scales flow. Under low wind conditions, the warmer and more convective UBL can form thermal circulations (Wang, 2009), or even a thermal low (Varentsov et al., 2018). Under more moderate and strong wind speed, the UBL can form a plume that transports heat and turbulent kinetic energy over rural areas downwind. Thus, the conditions within the UBL can also affect the structure and dynamics of the surrounding rural boundary layer and vice versa (Chen et al., 2012).

The numerous interactions between the urban surface, the overlying UBL and the various meso-scale flows (i.e. thermal circulations, see-breezes, low-level jets) indicate that understanding the feedback mechanisms that exist between them is essential to develop a solid understanding of the UBL dynamics (Barlow, 2014). Therefore, this thesis focus is to identify and quantify the interactions between the urban surface and the boundary layer in terms of surface energy balance and boundary-layer dynamics.

\subsection{Modelling the urban surface}

As discussed before, the urban areas alter the surface energy balance and affect the boundary-layer dynamics and meso-scale flows around them. These interactions have a direct impact on the health and well-being of people living in cities (Laaidi et al., 2012). Therefore, it is essential to model the physical processes that occur in urban areas to be able to better forecast weather in cities and issues early warnings for extreme weather events. Representative observations for entire urban areas are difficult to obtain, as the heterogeneity in the urban environment would require multiple measurement sites within a single city (Barlow, 2014). Consequently, combining model and observations can lead to a better understanding of the urban surface energy and the UBL.

\subsubsection{Urban canopy models}

Over the years different parametrization schemes, which represent the physical processes in the urban environment, have been developed. A parameterization scheme is a method to represent physical processes that occur on scales smaller than the model grid resolution 


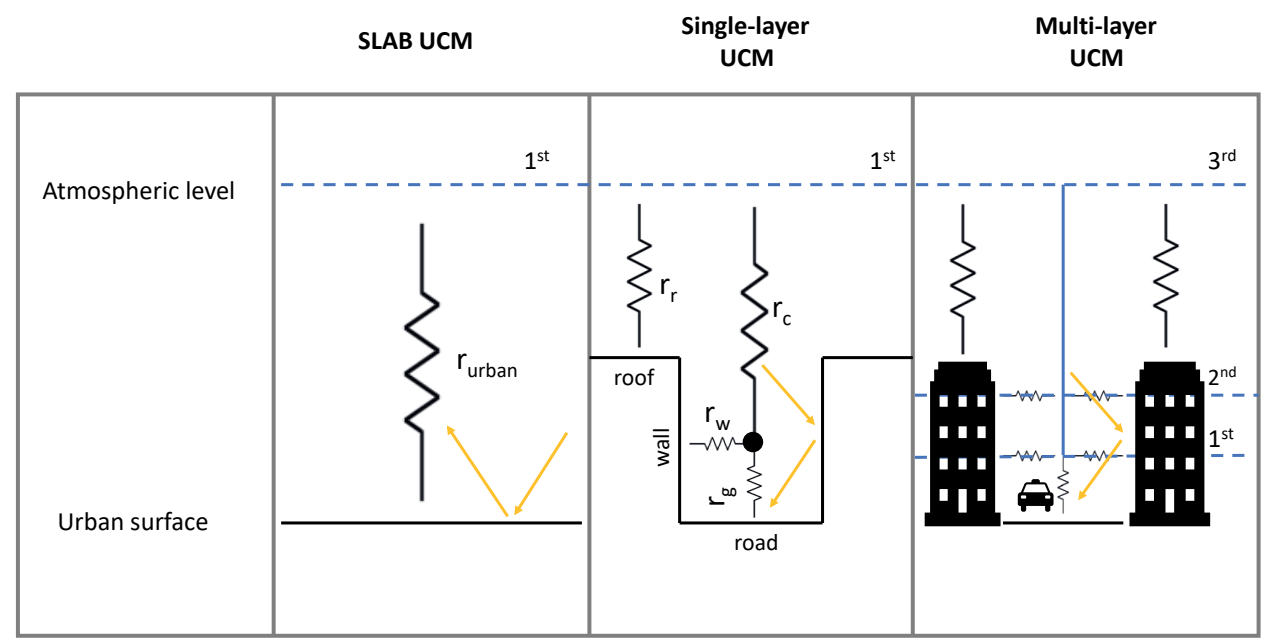

Figure 1.4.1: Conceptual sketch of the different complexity between UCMs and their coupling with the atmosphere. The link between the surface and atmosphere is illustrated using resistances $(r)$ for the urban area, the individual facets (roof $-r_{r}$, wall $-r_{\text {wall }}$, road $-r_{\text {ground }}$ and the urban canyon $\left(r_{c}\right)$. Difference in radiation processes is depicted with the yellow arrows.

(sub-grid scales) and therefore these processes cannot be explicitly resolved. The urban surface physical processes occur at the building scale $(<10 \mathrm{~m})$, but horizontal model resolutions usually exceeds $100 \mathrm{~m}$ (with a few exceptions). Therefore, it is essential to parametrize them. The urban surface parameterization schemes are commonly known as urban canopy models (UCMs) and come with varying degrees of complexity, depending on the physical processes they can represent (Figure 1.4.1).

In the simplest UCMs, known as slab models, the urban area is treated as "flat surface" (i.e. a land-use tile) with aggregated surface parameters (i.e. albedo, roughness length) representative for the urban area. These surface parameters are used to calculate radiation absorption and emission from the surface (i.e. albedo, emissivity), turbulent exchange with the atmosphere (i.e. roughness length) and heat storage in the urban fabric (i.e. thermal capacity, conductivity). Note that the morphology of the urban area (i.e. building height, canyon length) is not treated by slab models, but is indirectly accounted for by the prescription of a roughness length. Although some recent slab models are able to include more complex physical processes (e.g. Jarvi et al. (2011)), due to their simplicity cannot account for processes that occur within the urban canyon (i.e. radiation trapping, heat fluxes from walls or roads).

To account for these processes, single-layer urban canopy models are used (Masson, 2000; 
Kusaka et al., 2001; Harman et al., 2004; Porson et al., 2010; Chen et al., 2011a). These models are more complex. They require morphological parameters for the urban area (building height, road width, roof width, etc.) and individual surface parameters (albedo, thermal conductivity, heat capacity) for the roof, wall and road facets (Loridan et al., 2010). Therefore, they can simulate energy transfer and fluxes at all facets and can take into account the internal conditions within the buildings (Figure 1.4.1). Many of them also allow for the explicit calculation (or prescription) of the anthropogenic heat flux $\left(Q_{f}\right)$. Some of them can also represent water retention in urban facets after rainfall and the subsequent evaporation or removal through surface run-off (Yang et al., 2015, 2016). However, these models are coupled to the overlying atmosphere directly above the mean building height, which means that the surface energy fluxes from the different facets are added to the atmospheric model level closest to the urban surface. In these models, the effect of the urban morphology is indirectly represented through surface roughness lengths, which means that the effect of building drag on the turbulent flow is not explicitly represented. Therefore, single-layer UCMs have certain limitations and can lose detail when simulating air properties and turbulent exchange within the urban canyon (Baklanov et al., 2016).

To circumvent this deficiency, multi-layer urban canopy models can be used (i.e. Martilli et al. (2002) and Salamanca \& Martilli (2009)). These models allow for the urban surface to interact with multiple atmospheric model levels (Figure 1.4.1). This leads to a better turbulent exchange within the canyon and an explicit representation of building drag on the air flow. However, these models are more computationally expensive and require adjustments on the prognostic equations for heat, moisture and momentum in the atmospheric model (Baklanov et al., 2016).

Each UCM type is suited for different tasks, depending on the research goals/objectives. Simpler UCMs that require less surface parameters and are computationally inexpensive are more suitable for long term (yearly or even decadal) simulations or as tools for policy advice (Ward et al., 2016). Their low computations costs make them suitable for climate simulations, where the representation of spatial heterogeneity in the urban areas is not essential, given the low horizontal resolution of climate models. Single-layer and multilayers UCMs are more suited for coupling with atmospheric models (Baklanov et al., 2016). The former are widely used to predict turbulent exchange and weather over urban areas or to study interactions between the urban surface and meso-scale flows over moderately heterogeneous cities. The use of a multi-layer UCM is preferred, when there is a need to simulate weather conditions and turbulent exchange within the urban canyon, or when an urban area has a substantial amount of tall high-rise ( $>40-50 \mathrm{~m}$, i.e. Hong Kong) creating overlapping IBLs. 


\subsubsection{The coupling between urban canopy models and atmospheric mod- els}

UCMs are often used as standalone research tools (Grimmond et al., 2010; Loridan et al., 2010; Grimmond et al., 2011) in what is called an "off-line" mode, mainly for model evaluation purposes or for simulation of lengthy periods ( $>10$ years). However, UCMs are also coupled to atmospheric models for either research processes (i.e studying physical phenomena) or to provide forecasts for weather in urban areas. As is the case with UCMs, the complexity of the atmospheric model can also vary depending on the research purpose.

Less complex single-column models (SCMs or 1D models) have been used to study landatmosphere interactions over vegetated and urban surfaces (Sterk et al., 2013; Bosveld et al., 2014b; Song \& Wang, 2015; Nemunaitis-Berry et al., 2017; Tsiringakis et al., 2020). They usually represent a vertical column of a 3D-atmospheric model, where all parameterization schemes for various physical processes in the 3D-model (i.e. microphysics, boundary-layer dynamics, radiation) are active. This allows for a direct coupling between the surface and the atmosphere, which is essential to study land-atmosphere feedback mechanisms (Sterk et al., 2013; Tsiringakis et al., 2019). The SCMs also allow for more control over external forcing (i.e. advection) compared to 3D-atmospheric models, which makes them more suitable for investigating local land-atmosphere interactions and their effects on boundary-layer dynamics. However, they have certain limitations as they cannot resolve surface heterogeneity and thus are not well suited to study interaction between urban areas and meso-scale flows.

Urban canopy models are commonly coupled to 3D atmospheric models, also know as numerical weather prediction (NWP) models. Coupled NWP-UCM models have been widely used to study the impact of urban areas on the surface energy balance and boundary-layer dynamics (Loridan et al., 2013; Demuzere et al., 2017; Ferrero et al., 2018), urban heat islands (Ronda et al., 2017) and other meso-scale phenomena (Chen et al., 2011b). They allow for a representation of the urban surface heterogeneity via the use of different landuse classes, each with representative surface properties for different types of urban areas (Stewart \& Oke, 2012; Chen et al., 2011a). Topographic heterogeneity (i.e. mountains, coastlines, lakes) is also represented in NWP models, which allows to study interactions between urban areas and topographic meso-scale flows (i.e. katabatic winds). These fully coupled models offer a direct link between urban surface processes and meso-scale flows. However, due to their complexity and non-linearity, it is often difficult to study the local land-atmosphere interactions in detail.

There are two other types of 3D-atmospheric models that are used to study interactions between the urban surface and the overlying atmosphere. These are the large-eddy simulation (LES) models and the direct numerical simulation (DNS) models. The latter, although commonly used to resolve flow and turbulence in urban areas, usually lack the 
effects of heating on the turbulent flow and are computationally very expensive (Baklanov et al., 2016). Therefore, they are not described or used in this thesis. Largeeddy simulation (LES) models can resolve large scales of turbulent transport (typically $>5-10 \mathrm{~m}$ ), convection and clouds, which makes them ideal research tools for studying land-atmosphere interactions. They are also suitable to study urban-mesoscale flow interaction and boundary-layer dynamics (Wang, 2009). However, LES models are also very expensive and only recently have they been able to explicitly resolve the urban surface processes and heterogeneity (Resler et al., 2017). Thus in this thesis, nearly all types of atmospheric models (except DNS models) are used to study the different aspects of urban surface-atmosphere interactions and the coupling between the urban surface, the urban boundary layer and various mesoscale flows.

\subsection{The coupling between the urban surface, urban boundary layer and meso-scale flows}

The main research aim of this thesis is to study the coupling between the urban surface, the urban boundary layer and the meso-scale flows, and its effect on the turbulence exchange and the surface energy balance over urban areas. Observations that span the entire depth of the UBL are very limited (Barlow, 2014). Moreover, Ferrero et al. (2018) showed that the coupling between the UCM and the boundary-layer schemes is essential for the correct quantification of the turbulent exchange between the surface and the UBL. Therefore, it is essential to use a UCM coupled to an atmospheric model to study the interactions between the urban surface, UBL and the meso-scale flows.

\subsubsection{The role of urban surface-atmospheric feedback mechanisms}

We often mentioned that urban areas are heterogeneous environments, with a lot of spatial variability in building heights and shapes, construction materials and anthropogenic activities (Cadenasso et al., 2007). As atmospheric models increase in horizontal resolution, even down to 100m (Ronda et al., 2017; Lean et al., 2019), the representation of surface heterogeneity becomes increasingly important for the quantification of the surface energy balance and the accurate representation of the UBL (Arnfield, 2003; Barlow et al., 2015). Therefore, models need increasingly higher resolution inputs for the urban surface parameters (i.e. building height, surface albedo maps). The benefits of an increasingly accurate surface parameter description on model performance has been highlighted in previous UCM inter-comparison projects (Grimmond et al., 2010; Grimmond et al., 2011) and studies (Loridan et al., 2010; Loridan \& Grimmond, 2012a).

Despite the recent progress in deriving some of the surface parameters (Kent et al., 2018) and grouping them in many urban land-use classes (Stewart \& Oke, 2012; Demuzere et al., 2019), large uncertainties still exist in their spatial distribution and actual values. 


\subsection{The coupling between the urban surface, urban boundary layer and meso-scale flows}

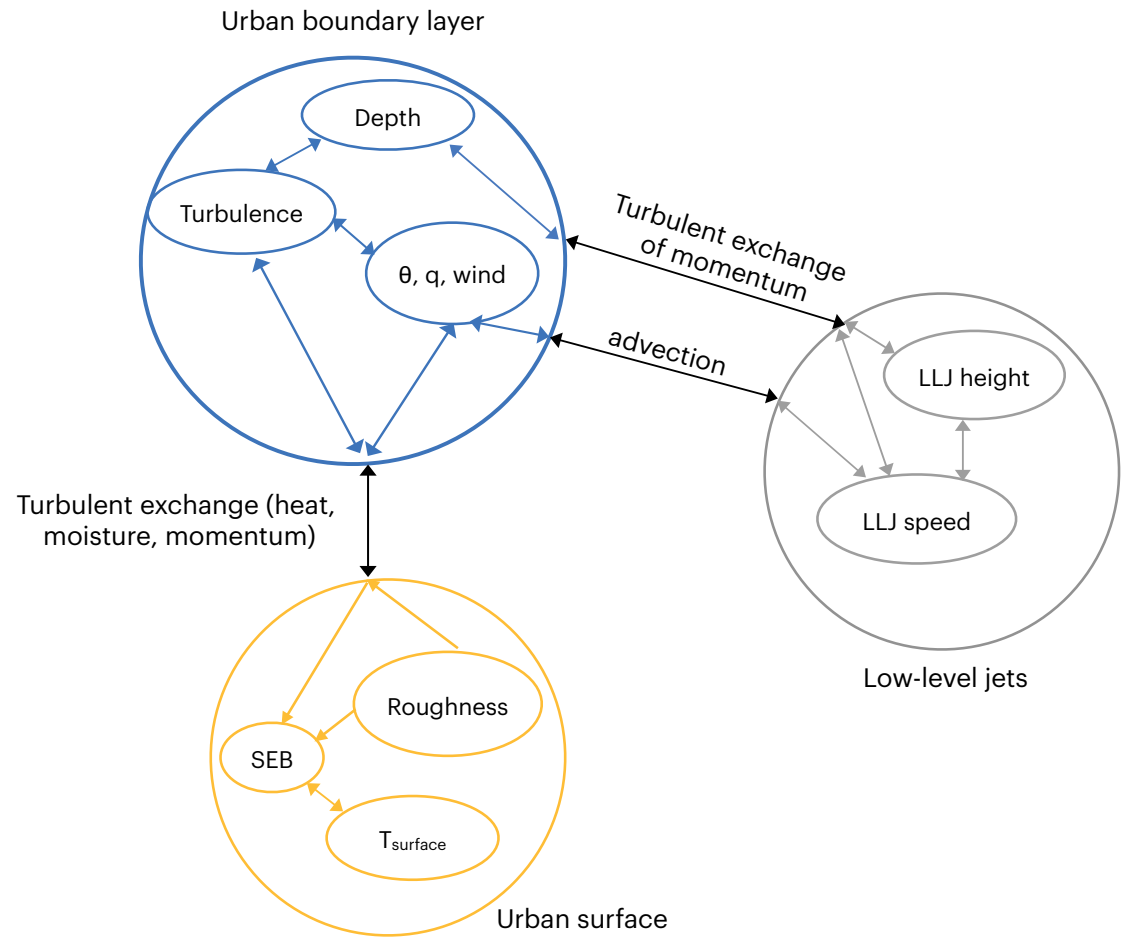

Figure 1.5.1: Conceptual figure of the interactions between the urban surface (orange), the urban boundary layer (blue) and the low-level jets (grey). Key state variables for each of components are illustrated with the smaller circles. Internal processes are depicted with single or double direction arrows, depending on whether it is a one- or two-way interaction. Interactions between the 3 components are illustrated with the black arrows.

However, there are also indirect ways to derive these uncertain surface parameters. One of the most common approaches is to use various model optimisation techniques, that aim at minimising model errors for certain energy balance variables (i.e. $Q^{*}, Q_{H}$ ). In this way, the surface parameters can be "optimized" to minimize model error. These approaches have been widely used in either "off-line" UCMs (Loridan et al., 2010; Wang et al., 2011; Loridan \& Grimmond, 2012a) or UCMs coupled with SCMs (Song \& Wang, 2015; Nemunaitis-Berry et al., 2017).

However, there is a risk involved with optimizing surface parameters in such a way and then implementing the optimized surface parameters in 3D atmospheric models. In the "off-line" UCMs, the changes in the surface energy balance do not affect the boundarylayer dynamics. Considering that the surface energy fluxes depend on the vertical profiles 
of temperature, moisture and wind, excluding the boundary-layer feedback might lead to a skewed/inaccurate optimization (Tsiringakis et al., 2019). On the other hand, UCM coupled with atmospheric models do include these feedback mechanisms, but cannot distinguish whether the changes in surface energy balance are mainly a result of surface parameter changes or the variations in UBL dynamics. Therefore, there is a need to quantify the atmospheric feedback effect for both coupled and "off-line" UCMs, to be able to better estimate surface parameters values and gain an better understanding of the coupling between the urban surface and the overlying boundary layer. This knowledge gap leads to the first research question of this thesis:

\section{What is the effect of urban surface-atmospheric feedback mechanisms} on the surface energy balance over cities?

\subsubsection{Uncertainty in the external atmospheric forcing}

A UCM is dependent on external atmospheric forcing (i.e. temperature, wind, moisture, shortwave radiation) to compute the radiative fluxes and the surface energy balance. When the UCM is coupled to an atmospheric model, the external atmospheric forcing is not derived from observations, as is the case in an "off-line" UCM, but is provided by other parts of the atmospheric model (i.e. boundary-layer scheme, radiation scheme).

External model bias, especially generated by errors in the boundary-layer scheme or due to erroneous advection estimations from nearby rural areas, can affect the model's ability to accurately calculate the surface energy balance and other related phenomena (i.e. UHI) over urban areas (Chen et al., 2012). However, the boundary layer is not the only external source of bias. Errors in shortwave radiative transfer, due to high aerosols concentration in the UBL (Gomes et al., 2008) or bias in longwave radiation (Kleczek et al., 2014), can easily affect the modelled surface energy balance. Therefore, it is essential to quantify these uncertainty sources and compare them to the variability in the surface energy balance due to uncertainty in the urban surface parameters. This knowledge gap leads us to the second research question:

What is the role of uncertainty in the external atmospheric forcing on the urban surface energy balance? 


\subsubsection{Meso-scale effects on the turbulent exchange in the urban boundary layer}

The turbulent exchange between the surface and the UBL is not only dependent on the surface forcing, but also on external meso-scale flows (i.e. sea-breeze, low-level jet) (Chen et al., 2012; Barlow, 2014). Horizontal advection associated with sea-breeze strongly influences the surface sensible and latent heat fluxes and the UBL structure over coastal cities (Lemonsu et al., 2006; Pigeon et al., 2007; Barlow, 2014). Low-level jet (LLJ) is another meso-scale phenomena that influences the structure and turbulent kinetic energy in the nocturnal boundary layer (Banta et al., 2006; Lundquist \& Mirocha, 2008; Halios \& Barlow, 2018).

The ability of a coupled UCM-atmospheric model to capture accurately the turbulent exchange over urban areas is strongly dependent on its ability to capture these mesoscale flows. However, meso-scale flows are also affected by urban surface roughness and surface heating (Yoshikado, 1992; Barlow, 2014; Barlow et al., 2015). Correctly modelling these interactions is essential to accurately represent the turbulent exchange in the UBL and is also relevant for predicting air quality in urban areas (Banta et al., 2004; Barlow, 2014).

Here, one of these interactions is studied, namely the interaction between low-level jets and the urban boundary layer. This choice is made for three reasons. First, the interaction between low-level jets and urban areas is less commonly studied with the use of atmospheric models, than the interaction between urban areas and sea-breeze circulations (Barlow, 2014). Moreover, sea-breezes are strongly related to advection of heat and moisture in the UBL (Pigeon et al., 2007) and as such these interactions are indirectly studied in the second research question. Finally, the urban area-LLJ interactions occur mainly during the night. This will allows us to elucidate part of the turbulent exchange in the nocturnal UBL, which is more complex than its daytime counterpart (Barlow et al., 2015). These knowledge gaps lead to the third research question:

\section{How does a low-level jet interact with the urban boundary layer?}




\subsection{Thesis Outline}

The aim of this thesis is to study the coupling between the urban surface, the boundary layer and meso-scale flows, and its effect on the surface energy balance and turbulent exchange in the UBL, by answering the three research questions posed before. Chapter 2 is focused towards the first research question, chapter 3 answers the second one research question, while chapters 4 and 5 answer the last research question.

In Chapter 2, a comparison of a UCM's response to changes in uncertain surface parameters is conducted for an "off-line" and "on-line" UCM (i.e. coupled to a 1D atmospheric model). For this comparison we use the SUBLIME case study (Steeneveld et al., 2017), which covers a period of two clear-sky summer days over London, to minimise the effects of clouds and rain on the surface energy balance. Here, we quantify the effect of the feedback mechanisms between the urban surface and the UBL on the surface energy balance. In this way we also develop a better understanding of the coupling between the surface and the UBL, before proceeding further into the following research questions.

In Chapter 3, the previous analysis is expanded to include the sensitivity of a UCM to changes in the external atmospheric forcing. This research utilises a coupled UCM-1D atmospheric model setup and the same case study period as chapter 2. The results reveal the importance of biases in external atmospheric forcing on the UCM performance and their effect on the surface energy balance and turbulent exchange in the UBL.

Chapters 4 and 5 focus on the interactions between low-level jets and urban areas. Here, we aim to quantify the effects of cities on the LLJ occurrence frequency and characteristics (i.e. height, speed). Moreover, we study the LLJ effects on the turbulence in the nocturnal UBL. Chapter 4 is based on a real case study between the 14-16 May 2019 over London, utilising both observations and two 3D atmospheric models. In chapter 5, the state-of-art PALM LES model (Maronga et al., 2020) is used in combination with our previous UCM1D atmospheric model setup to simulate the evolution of a low-level jet in an idealised case study, based on the second night (15-16) of the May 2019 case study period.

In Chapter 6, the findings of all previous chapters are synthesised to highlight the key research findings of this thesis. Limitations of the current thesis and future recommendations for interesting research topics are discussed. The supplementary material for each research chapter are provided in the Appendix. 


\section{Chapter 2}

\section{On- and off-line evaluation of the single-layer urban canopy model in London summertime conditions}

This chapter is based on:

Tsiringakis, A, Steeneveld, G-J, Holtslag, AAM, Kotthaus, S, Grimmond, S. (2019) Onand off-line evaluation of the single-layer urban canopy model in London summertime conditions. Q J R Meteorol Soc. 145: 1474- 1489. https://doi.org/10.1002/qj.3505 


\section{Abstract}

Urban canopy models are essential tools for forecasting weather and air quality in cities. However, they require many surface parameters, which are uncertain and can reduce model performance if inappropriately prescribed. Here, we evaluate the model sensitivity of the single-layer urban canopy model (SLUCM) in the Weather Research and Forecasting (WRF) model to surface parameters in two different configurations, one coupled to the overlying atmosphere (on-line) in a 1D configuration and one without coupling (off-line). A two-day summertime period in London is used as a case study, with clear skies and low wind speeds. Our sensitivity tests indicate that the SLUCM reacts differently when coupled to the atmosphere. For certain surface parameters, atmospheric feedback effects can outweigh the variations caused by surface parameter settings. Hence, in order to fully understand the model sensitivity, atmospheric feedback should be considered. 


\section{$2.1 \quad$ Introduction}

More accurate urban weather forecasts for human thermal comfort, air quality, energy demand and wind in urban areas are necessary for the vast urban population, which is expected to be $66 \%$ of the world's population by 2050 (UN, 2014). These forecasts require a good understanding of the physical and chemical processes in the urban boundary-layer (UBL) (Barlow, 2014).

Understanding the turbulent transport is essential for a correct quantification of the surface energy balance in urban areas (Pigeon et al., 2007). Building height, shape and materials are linked to different surface properties (e.g. albedo, thermal conductivities and heat capacities). Correctly modelling the physical processes in the urban environment depends on the adequate representation of surface properties (Grimmond et al., 2011; Best \& Grimmond, 2015). For example, short-wave radiation at building facets is governed by albedo, and radiation trapping in urban canopy varies with canyon morphology and surface emissivity (Best \& Grimmond, 2015). Moreover, surface albedo, emissivity, heat capacity and thermal conductivity of the building materials directly influence heat storage in the urban fabric during day-time and its consequent release to the atmosphere at night.

To provide forecasts for urban environments, numerical weather prediction (NWP) models usually utilize a so-called urban canopy models (UCM) (Masson, 2000; Martilli et al., 2002; Best, 2005; Chen et al., 2011a). These incorporate the sub-grid scale physical processes of the urban environment and the complexity and heterogeneity of the urban surface. The required surface parameters remain a challenge to define appropriately. While mean building height and other morphological parameters are generally well represented (Kent et al., 2018), other surface properties like albedo, emissivity, thermal conductivity and heat capacity are very uncertain. If the parameters settings do not accurately represent the urban environment to-be-modelled, this can hinder model performance (Grimmond et al., 2010; Grimmond et al., 2011).

Recent studies have investigated the sensitivity of urban canopy models to these surface parameters, but mainly in off-line mode (no coupling with the overlying UBL). For example Loridan et al. (2010), Wang et al. (2011) and Zhao et al. (2014) did comprehensive analysis on the effects of surface parameters in NOAH-SLUCM off-line. They found that the accuracy in modelled surface energy balance fluxes heavily depends on the correct values for the urban surface parameters. Similar conclusions where reached by the PILPS-urban inter-comparison experiment (Grimmond et al., 2010; Grimmond et al., 2011), where various urban surface parametrization schemes were evaluated. They concluded that the complexity, with which the UCMs parametrize the physical processes in the urban environment is not as important as the correct prescription of the surface properties. 
Although the off-line modelling studies are valuable for improving UCMs, they represent a simplified test setting as feedback mechanisms with the overlying atmosphere are not accounted for. Coupling of UCMs to the overlying atmosphere is thus essential to understand the effects of surface parameters on model performance and can assist in selection of surface parameters that lead to more robust results for specific case studies and for future forecasts purposes (Song \& Wang, 2015).

Interactions with the overlying atmosphere in a NWP model can either be coupled to a surface model in a full 3D set-up or in a 1D set-up (single column model). Many studies have investigated the effects of surface parametrization on boundary-layer representation (Pigeon et al., 2007; Flagg \& Taylor, 2011; Ferrero et al., 2018), surface energy balance (Pigeon et al., 2007; Loridan et al., 2013; Demuzere et al., 2017), urban heat islands (Miao et al., 2009; Bohnenstengel et al., 2011; Nemunaitis-Berry et al., 2017; Ronda et al., 2017) or other mesoscale phenomena (Chen et al., 2011b). The majority of the studies uses a 3D set-up, which limits the ability to link model response to changes in surface parameters and investigate feedback mechanisms in detail. To circumvent this drawback the 1D setup can be used (Song \& Wang, 2015, 2016; Nemunaitis-Berry et al., 2017), which allows for detailed investigation of model response to changes in surface parameters, while at the same time having much lower computational costs compared to the 3D set-up.

Evaluation of a UCM (off-line, on-line 1D or 3D) to changes in surface parameters may yield different results depending on the atmospheric conditions imposed and the strength of the atmospheric feedback mechanisms. On-line analysis should provide more realistic results of the sensitivity of the surface energy balance to changes in surface parameters, but it needs to be used in combination with an off-line analysis to identify the effect of feedback mechanisms. To our knowledge, only a couple of studies present such a sensitivity analysis (Loridan et al., 2013; Nemunaitis-Berry et al., 2017). Hence, to date, effects of the feedback mechanisms on the surface energy balance have not yet been sufficiently quantified. It is not clear if the effect of the on-line coupling can outweigh the effects of changes in surface parameters or under which conditions the off-line evaluation is sufficient to understand model behaviour.

To investigate the effects of the feedback mechanisms on the model performance, both an off-line version of the Noah-SLUCM model and a on-line version 1D-WRF-Noah-SLUCM are used. The case study set-up and forcing (section 2.2), as well as methodology and model set-up (section 2.3) are described. Model evaluation (section 2.4), atmospheric feedback mechanisms (section 2.5) and the sensitivity to changing surface parameters (section 2.6) are presented prior to discussion (section 2.7) and conclusions (section 2.8). 


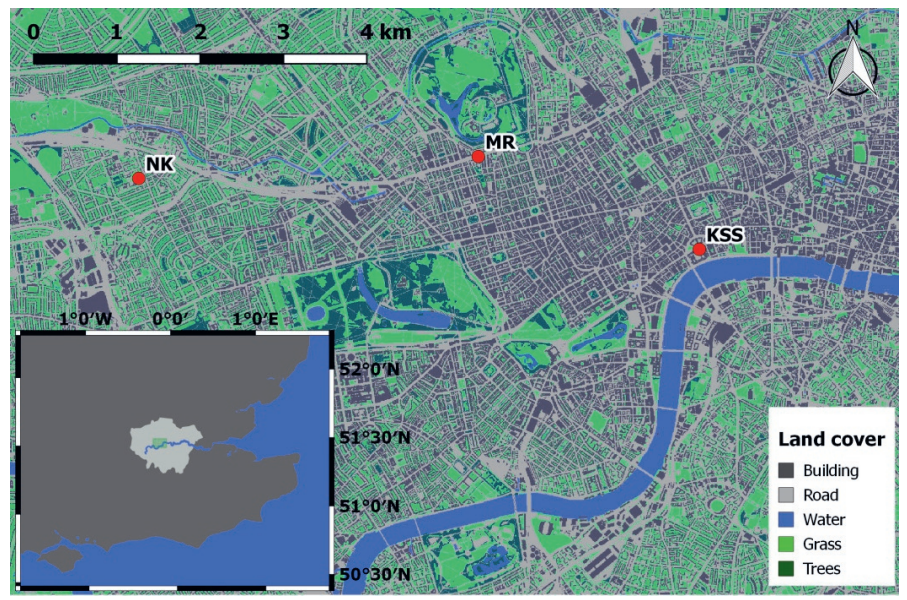

Figure 2.2.1: Land cover map (based on OS MasterMap) of central London study area with measurement site locations. Inset shows study area within Greater London in South-east England (source (Steeneveld et al., 2017))

\subsection{Description of case study}

Off-line and on-line models are evaluated, using observations in the dense city centre of London, UK, at the King's College London measurement site (KSSW Figure 2.2.1). Air temperature, wind, radiation and surface fluxes measured at $50 \mathrm{~m}$ above ground level are used (for details see Kotthaus \& Grimmond, 2014a). Mixed-layer height (MLH) for central London is derived from ceilometer measurements at the MR site (Figure 1; Kotthaus \& Grimmond, 2018). Comparing MLH results for two sites within central London at a distance of $4 \mathrm{~km}$ (NK, Figure 2.2.1), Kotthaus et al. (2018) found spatial variations of MLH to be mostly within the uncertainty of MLH measurements

The area around KSSW site has been extensively described by Kotthaus \& Grimmond (2014b), including bulk albedo, urban land cover fractions and roof/road fractions. Unmeasured parameters are assigned based on literature including: a) thermal conductivities and heat capacities for the buildings in the study area, which are based on existing literature regarding material composition of buildings in central London (Oikonomou et al., 2012) and building material thermal properties (Engineering Toolbox, 2010) and b) emissivities based on Bohnenstengel et al. (2011). All the aforementioned derived parameters are used instead of the Chen et al. (2011a) high density residential 'default' values (Table 2.3.1).

To evaluate the model response to surface parameter settings, 'simple' atmospheric conditions are chosen (i.e. avoiding clouds, rainfall and high wind speed). Following a detailed evaluation of a long-term measurement period (2010-2015) at KSS, a 2 day period (23-25 
July 2012) was selected. During these days a moderate heat wave (nearly $30^{\circ} C$ ) occurred in London, with relatively low wind speeds $\left(<6 \mathrm{~m} \mathrm{~s}^{-1}\right.$ at $\left.50 \mathrm{~m}\right)$, no clouds or rain and moderate temperature advection.

KSSW observations of air temperature, wind speed, wind direction, pressure, as well as short-wave and long-wave downward radiation fluxes at $50 \mathrm{~m}$ above ground level are used to drive the off-line model. For the 1D set-up, initial profiles of wind, potential temperature $(\theta)$, moisture and surface pressure are prepared from the nearest radio-soundings (UWYO, 2012) at Herstmonceux, Hailsham, UK, nearly $70 \mathrm{~km}$ south-east of London at 0000UTC and the KSSW measurements. Large-scale forcing for wind and geostrophic wind is derived from ECMWF operational reanalysis data (ECMWF, 2012) in combination with a WRF simulation conducted for the case study period. Temperature and moisture advection are derived from 3D-WRF model simulations for London and synthesized with in-situ measurement of surface advection from WMO stations (NOAA/NCDC, 2012) within and around London. Initial soil temperature and moisture profiles for both set-ups are taken from 3D-WRF simulation (spun-up for 12 hours) and then cycled $3 x 2$ days in the off-line set-up, until the deeper soil temperature became constant and ground heat flux shows a similar daytime range for both days of the case study. A detailed description of the case study can be found in the call for participation in the Singlecolumn Urban Boundary Layer Inter-comparison Modelling Experiment (SUBLIME) of Steeneveld et al. (2017).

\subsection{Methodology}

\subsubsection{Model set-up}

In both the on-line and off-line cases, the urban surface is represented using the single-layer UCM (Kusaka et al., 2001; Chen et al., 2011a) and for surface layer parametrization the Mello-Yamada-Janjic (MYJ) scheme (Janjic, 1994). For the land surface the NOAH-LSM version 3.4.1 Chen \& Dudhia (2001) is used. The on-line simulation uses the WRF model version 3.8.1 (Skamarock et al., 2008) in a single-column format. The 2.5 order MYNN scheme (Mikio \& Hiroshi, 2009) is used as boundary layer parametrization, which is well tested in combination with the SLUCM and the MYJ surface-layer scheme. For both short-wave and long-wave radiation RRTMG schemes (Iacono et al., 2008) are applied. As no cloud or rain occurred during the case study period, the simple WSM 3rd-class scheme of Hong et al. (2004) is chosen for the micro-physics representation.

Both models run for 54 hours in total starting at 0000 UTC 23 July 2012 and finishing at 0600 UTC 25 July 2012. For the model evaluation (section 2.4) the 54-hour period is used. For the sensitivity analysis the first 6 hours are considered spin-up time to allow for surface parameter changes to have an effect on model response. The off-line set-up is forced at each model time step (30 minutes) with the observations (section 2.2). The on-line set-up 
uses initial soil moisture and temperatures profiles up to a depth of $1.5 \mathrm{~m}$ and atmospheric profiles (moisture, $\theta$ and wind) up to $17 \mathrm{~km}$. The advection forcing is prescribed in the 100-250 m layer and then linearly decreased to 0 at the surface and at $1000 \mathrm{~m}$. Potential temperature, moisture and momentum advection tendency term is applied at each timestep $(30 \mathrm{~s})$ of the on-line model and changes every 6 hours. Geostrophic wind is prescribed in the initial time step above $1 \mathrm{~km}$ and then is evolving in time via a tendency term that is applied each time-step for all model levels. More detailed description of the forcing data for the 1D-WRF can be found in Steeneveld et al. (2017).

\subsubsection{Urban canopy model description}

The single-layer urban canopy model (SLUCM) of WRF (Kusaka et al., 2001; Chen et al., 2011a) is coupled to the NOAH land-surface scheme. It uses a tile approach with one patch representing the urban surface handled by the SLUCM, while vegetation is handled by the NOAH-LSM. Surface radiation and turbulent fluxes are calculated for each tile and averaged according to land-cover fractions. The same holds for surface temperatures and surface albedo.

The SLUCM urban morphology uses a 2D canyon approach, without street orientation or varying building heights. The urban patch is split into 3 facets (roof, wall, road), each receiving a normalized contribution of the total urban fluxes. The plan area consists of roof $\left(F_{\text {roof }}\right)$ and the road $\left(F_{\text {road }}\right)$. The wall fraction $\left(F_{\text {wall }}\right)$ is calculated as:

$$
F_{\text {wall }}=2 \overline{z_{h}} /\left(W_{\text {roof }}+W_{\text {road }}\right)
$$

Here $\overline{z_{h}}$ is the mean building height $(\mathrm{m})$ and $W_{\text {roof }}$ and $W_{\text {road }}$ are the roof and road widths (in $\mathrm{m}$ ). Each surface flux $\left(\mathrm{Q}, W m^{-2}\right)$ is calculated as (Kusaka et al., 2001) :

$$
\begin{gathered}
Q_{\text {urb }}=F_{\text {roof }} Q_{\text {roof }}+F_{\text {road }} Q_{\text {road }}+F_{\text {wall }} Q_{\text {wall }}, \\
Q_{\text {total }}=f_{\text {urb }} Q_{\text {urb }}+\left(1-f_{\text {urb }}\right) Q_{\text {nat }},
\end{gathered}
$$

Where the total flux from the surface $\left(Q_{\text {total }}\right)$ is based on the urban fraction $f_{\text {urb }}$ and the vegetation fraction $\left(1-f_{u r b}\right)$ and their respective fluxes $\left(Q_{u r b}, Q_{n a t}\right)$. The sky-view factor of each facet regulates radiation (short-wave and long-wave) received. Turbulent sensible heat $\left(Q_{H}\right)$ and moisture $\left(Q_{E}\right)$ fluxes from each facet are given by :

$$
\begin{gathered}
Q_{H}=\rho C_{P} C_{H} U_{a}(\Delta \theta), \\
Q_{E}=\rho L_{V} C_{E} U_{a}\left(q_{s k i n}-q_{a i r}\right),
\end{gathered}
$$

Where, $\rho$ is the air density $\left(\mathrm{kg} \mathrm{m}^{-3}\right), C_{P}$ is the specific heat capacity of dry air $\left(J \mathrm{~kg}^{-1} \mathrm{~K}^{-1}\right)$ and $C_{H}$ and $C_{E}$ are the exchange coefficients for heat and moisture. $\Delta \theta$ $(K)$ is the potential temperature gradient between the surface and the air. $L_{V}$ is the latent heat for vaporization $\left(\mathrm{J} \mathrm{kg}^{-1}\right) . U_{a}$ is the wind speed $\left(\mathrm{m} \mathrm{s}^{-1}\right)$ and $q_{\text {skin }}$ is the specific 
Table 2.3.1: Urban surface parameters, their minimum and maximum limits and the incremental changes per experiment. Default values indicate the values that each parameter retains, when another parameter is varied.

\begin{tabular}{ccccc}
\hline Parameters & minimum limit & maximum limit & incremental change & default values \\
$f_{\text {urb }}(0-1)$ & 0.60 & 1.00 & 0.05 & 0.85 \\
$\alpha_{\text {roof }}(0-1)$ & 0.05 & 0.40 & 0.05 & 0.18 \\
$\alpha_{\text {wall }}(0-1)$ & 0.05 & 0.40 & 0.05 & 0.20 \\
$\alpha_{\text {road }}(0-1)$ & 0.05 & 0.40 & 0.05 & 0.08 \\
$\lambda_{\text {roof }}\left(\mathrm{Js}^{-1} \mathrm{~K}^{-1}\right)$ & 0.15 & 1.20 & 0.15 & 0.30 \\
$\lambda_{\text {wall }}\left(\mathrm{Js}^{-1} \mathrm{~K}^{-1}\right)$ & 0.15 & 1.20 & 0.15 & 0.60 \\
$\lambda_{\text {road }}\left(\mathrm{Js}^{-1} \mathrm{~K}^{-1}\right)$ & 0.15 & 1.20 & 0.15 & 0.60 \\
$C_{\text {roof }}\left(\mathrm{Jm}^{-3} \mathrm{~K}^{-1}\right)$ & $0.30 * 10^{6}$ & $2.40 * 10^{6}$ & $0.30 * 10^{6}$ & $0.90 * 10^{6}$ \\
$C_{\text {wall }}\left(\mathrm{Jm}^{-3} \mathrm{~K}^{-1}\right)$ & $0.30 * 10^{6}$ & $2.40 * 10^{6}$ & $0.30 * 10^{6}$ & $1.50 * 10^{6}$ \\
$C_{\text {road }}\left(\mathrm{Jm}^{-3} \mathrm{~K}^{-1}\right)$ & $0.30 * 10^{6}$ & $2.40 * 10^{6}$ & $0.30 * 10^{6}$ & $1.50 * 10^{6}$ \\
\hline
\end{tabular}

humidity at the surface $\left(\mathrm{kg} \mathrm{kg}^{-1}\right)$, while $q_{\text {air }}$ is the atmospheric one. Each of the facet's fluxes is calculated separately and then averaged proportionally to the percentage contribution of each facet (namely $F_{\text {roof }}, F_{\text {road }}$ and $F_{\text {wall }}$ ). Anthropogenic heat flux of $38 \mathrm{Wm}^{-2}$ (with diurnal profile), based on yearly estimates for central London (Dong et al., 2017), is added to the first model level and incorporated into the sensible heat flux term.

Loridan et al. (2010) found that some of the most important surface parameters (based on their effect in model performance) are : a) urban fraction $\left(f_{\text {urb }}\right)$; b) albedo of roof $\left(\alpha_{\text {roof }}\right)$, wall $\left(\alpha_{\text {wall }}\right)$ and road $\left.\left(\alpha_{\text {road }}\right) ; \mathrm{c}\right)$ thermal conductivity $\left(\lambda_{\text {roof }}, \lambda_{\text {wall }}, \lambda_{\text {road }}\right)$ and d) heat capacity $\left(C_{\text {roof }}, C_{\text {wall }}, C_{\text {road }}\right)$ for each facet. To test the response in both model set-ups these parameters are varied individually (Table 2.3.1), while others were kept at their respective default value.

\subsubsection{Normalization of fluxes}

While the on-line model performs quite well, when default parameters are used (see section 2.4), some deviation remains between modelled and observed meteorological values. To account for the small differences in the atmospheric conditions between both set-ups, we follow Loridan \& Grimmond (2012a) approach and we normalize all energy fluxes with the total incoming radiation:

$$
\hat{Q}=Q /\left(S W_{\text {down }}+L W_{\text {down }}\right),
$$

Here $\hat{Q}$ is the normalized flux, $\mathrm{Q}$ is the original flux in $W m^{-2}, S W_{\text {down }}$ is the downward short-wave radiation flux and $L W_{\text {down }}$ is the downward long-wave radiation flux, both in $W m^{-2}$. Representing radiation and turbulent fluxes as a fraction of the total incoming radiation allows demonstration of how changes in surface parameters affect the distribution of incoming radiation between different components of the surface energy balance. Given 
there are no clouds in the the case study period, the daily average incoming radiation over these two days remains almost constant. However, in the on-line 1D-WRF-SLUCM cases changes in surface properties can affect the atmospheric temperature, which alters the $L W_{\text {down }}$ in response. The overall change in $L W_{\text {down }}$, due to surface changes is $<8 \mathrm{Wm}^{-2}$ for the 54 -hour period (i.e. $0.9 \%$ change for mean daytime total incoming radiation and $2.3 \%$ during night-time). Thus $L W_{\text {down }}$ remains almost constant.

Following Best \& Grimmond (2015), all fluxes are analysed based on the sign of the net radiation $\left(Q^{*}\right)$, with positive $Q^{*}$ indicating daytime and the remaining being nocturnal. This means the different response of the variables is tested under strong and weak turbulent regimes and the role of each surface parameter in day or night-time energy balance is investigated.

The mean incoming radiation flux is approximately $922 \mathrm{Wm}^{-2}$ during daytime and 355 $W m^{-2}$ during night-time in the on-line set-up. For simplicity, conversions from normalized to actual values (as presented in section 2.6), use these values for incoming radiation, despite small variations in response of the latter to changes in surface parameters (as discussed). The off-line modelled (and observed) mean incoming radiation fluxes are 905 $W^{-2}$ (daytime) and $357 \mathrm{Wm}^{-2}$ (night-time), and do not change with varying surface parameters.

\subsection{Model evaluation}

Model runs using the default parameter values are used as the reference. The variables evaluated are $Q^{*}, S W_{\text {down }}, L W_{\text {down }}$, turbulent sensible $\left(Q_{H}\right)$ and latent $\left(Q_{E}\right)$ heat fluxes, air temperature $\left(T_{\text {air }}\right)$ and wind components $(\mathrm{U}$ and $\mathrm{V}$ ) at $50 \mathrm{~m}$ and the mixed-layer height (MLH). The $S W_{\text {down }}$ and $L W_{\text {down }}$ fluxes and $50 \mathrm{~m}$ temperature and wind speed are the observations forcing the off-line model. Storage heat flux $\left(\Delta Q_{S}\right)$ is not directly observed. Although it could be derived as the residual of surface energy balance (as in Kotthaus \& Grimmond, 2014b), it is not analysed here given the accumulation of errors (Grimmond \& Oke, 1999) and the potential mismatch between the measurement footprint of the turbulent fluxes and the radiation fluxes (Schmid et al., 1991).

The modelled $S W_{\text {down }}$ (Figure 2.4.1b) has a mean bias of $10.8 \mathrm{Wm}^{-2}$ for the 2-days, with a maximum bias of $37.0 \mathrm{Wm}^{-2}$ around noon/afternoon. One source of this bias could be lack of sufficient aerosols loading in the model. Observational uncertainty, such as dust accumulated on the radiation sensor, could also cause a decrease in the short-wave radiation signal measured by the instrument. Given the expected radiometer accuracy of $\pm 10 \%$ of the measured values (Kotthaus \& Grimmond, 2014a), it is hard to identify the source of this deviation. The on-line $L W_{\text {down }}$ radiation is underestimated by up to $-14.0 \mathrm{Wm}^{-2}$, with a mean bias of $-4.0 \mathrm{Wm}^{-2}$. Reasons for this underestimation could be a cold bias in the vertical profile of temperature (compared to observations) or lack 

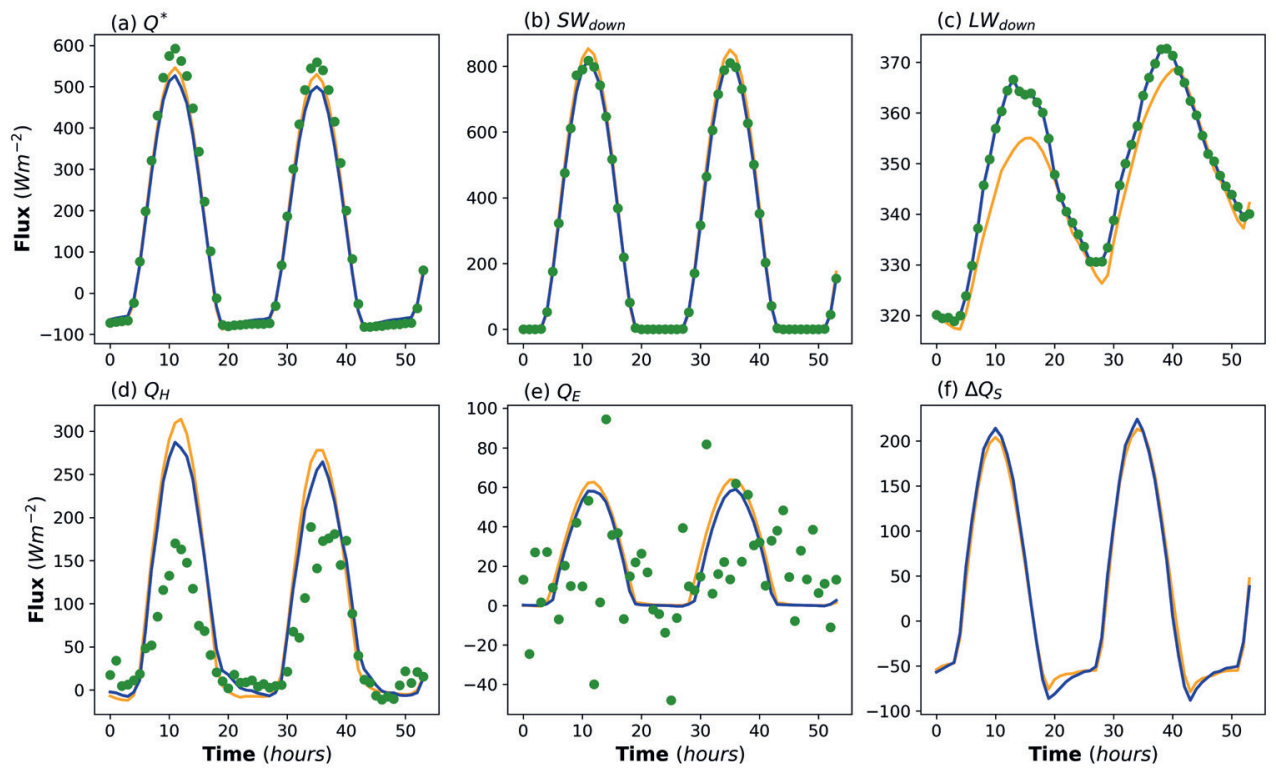

online $\quad$ offline

- observations

Figure 2.4.1: Evaluation of modelled radiation and surface energy fluxes, a) $Q^{*}$, b) $S W_{\text {down }}$, c) $L W_{\text {down }}$, d) $Q_{H}$, e) $Q_{E}$ and f) $\Delta Q_{S}$ against in situ observations at the KSSW station for the period 0000 UTC 23 July 2012 to 0600 UTC 25 July 2012. All flux units are in $W m^{-2}$.

of sufficient water vapour in the atmosphere. These model biases are most likely linked to a combination of inaccurate forcing and deficiencies of the parametrization scheme, according to Kleczek et al. (2014).

The on-line case generally underestimates $Q^{*}$ during the daytime up to $-49.8 \mathrm{Wm}^{-2}$, while at night there is a small overestimation by up to $11.0 \mathrm{Wm}^{-2}$, leading to a mean bias of -13.2 $\mathrm{Wm}^{-2}$ (Figure 2.4.1a). Off-line $Q^{*}$ has a daytime and night-time peak biases of $-66.0 \mathrm{Wm}^{-2}$ and $13.1 \mathrm{Wm}^{-2}$ respectively, resulting in a slightly larger mean bias of -19.6 $W m^{-2}$. These $Q^{*}$ model biases are similar in magnitude to those reported by Loridan et al. (2013) in their off-line and on-line modelling studies for the KSS site. One source of error for the daytime modelled $Q^{*}$ is the positive bias in modelled $S W_{u p}$ (up to 37.1 $\left.W m^{-2}\right)$ compared to the observed $S W_{u p}$. This indicates a potential overestimation in the bulk surface albedo, which could be caused by mismatch between the model description and the realistic physical description of the urban canopy in the radiometer source area. As Kotthaus \& Grimmond (2014b) state the effective albedo at this site varies both with solar elevation and azimuth angle (i.e. it changes in time). This effect is not captured in the model and could explain some of the error. The reflection effect in $Q^{*}$ is more evident between 1000-1600 UTC. Another constant error in $Q^{*}$ is linked to lower $L W_{\text {down }}$. 

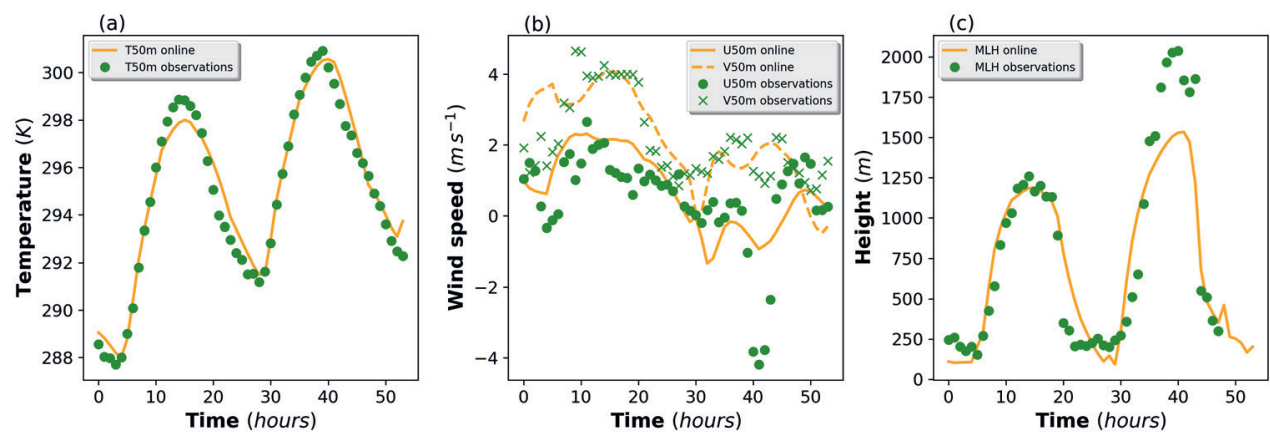

Figure 2.4.2: Evaluation of modelled (a) temperature (K), (b) U and V wind components $\left(\mathrm{m} \mathrm{s}^{-1}\right)$ at $50 \mathrm{~m}$ above ground level and (c) mixed-layer height (m) against in-situ observations at the KSSW and MR (MLH) sites (Figure 2.2.1) for the 0000 UTC 23 July 2012 to 0600 UTC 25 July 2012.

Finally, long-wave upward flux $\left(L W_{u p}\right)$ calculated from the model is contributing around $20 \mathrm{Wm}^{-2}$ of the negative bias during daytime, while it also increases $Q^{*}$ by $15 \mathrm{Wm}^{-2}$ during night-time.

All modelled turbulent fluxes have substantial deviations from the observed values. Online daytime $Q_{H}$ is usually overestimated (maximum bias of $150 \mathrm{~W} \mathrm{~m}^{-2}$ ) and underestimated at night-time (by up to $-44 \mathrm{~W} \mathrm{~m}^{-2}$ ). Overall the mean overestimation is 35.5 $W m^{-2}$ for the 2-days. The off-line cases has lower bias, with a mean overestimation of $32.7 \mathrm{~W} \mathrm{~m}^{-2}$ for the whole period. The observed fluctuations in $Q_{E}$ throughout the case study period, make the evaluation of the modelled $Q_{E}$ challenging. Previous studies for the KSSW area from Loridan et al. (2013) and Kotthaus \& Grimmond (2014b) have reported a similar variability in the measured $Q_{H}$ and $Q_{E}$. According to Kotthaus \& Grimmond (2014a) variability in turbulent transport near KSSW station could explain some of the variability in the observed fluxes. For wind directions $165^{\circ}$ to $205^{\circ}$, which predominates in this case study, they found that they observed $Q_{H}$ tends to be substantially lower compared to other wind directions. Changes in the source area or the general lower friction velocity due to the river Thames might have contributed to a reduction in $Q_{H}$ flux. Generally the source of the $Q_{E}$ for this site is primarily from past rainfall, as vegetation fraction is relatively low and the overall contribution from the river Thames (Kotthaus \& Grimmond, 2014a) is not significant. During dry days, such as this study period, the uncertainty of the small $Q_{E}$ flux is large. Moreover, the eddy co-variance uncertainty for the individual 30 minute values should be handled with care.

On-line modelled air temperature (at $50 \mathrm{~m}$ ) follows well the diurnal cycle seen in the observations (Figure 2.4.2a). A cold bias is observed around noon (around -1.0 K) followed by a warm bias of equal magnitude during late evening. The mean temperature bias is 0.2 
$K$. The deviation between modelled and observed temperature could have many sources linked to surface energy balance or larger scale temperature advection. Underestimation of modelled $Q^{*}$ or lower anthropogenic heat flux might also explain this. U and $\mathrm{V}$ wind components have mean biases of $0.20 \mathrm{~m} \mathrm{~s}^{-1}$ and $0.04 \mathrm{~m} \mathrm{~s}^{-1}$ (Figure 2.4.2b), respectively. Changes in wind speed and wind direction are captured well by the model with one exception. Between $39 \mathrm{hr}$ and $45 \mathrm{hr}$ in the model simulation a sudden change of wind direction occurs. This is linked to a sea-breeze intrusion over London (also found by Coceal et al., 2018). The 1D-WRF-SLUCM is unable to capture this event because the momentum advection is imposed in 6-hourly blocks.

The diagnosed MLH top (Figure 2.4.2c) in the on-line model is similar to observations on the first case study day, but underestimated by up to $500 \mathrm{~m}$ at around 36-44 hours in the simulation. Overestimations in diagnosed MLH occur primarily during later afternoon and early morning (up to $300 \mathrm{~m}$ ). Mean underestimation for the 2 days is $28 \mathrm{~m}$. The underestimation during the second day is likely caused by the prescribed negative temperature advection, which is imposed up to a height of $1000 \mathrm{~m}$ during daytime. This could potentially impose an artificial inversion which could be diagnosed as the MLH by the model.

\subsection{Feedback mechanisms between the surface and the overlying atmosphere}

Feedback mechanisms between the surface and the boundary-layer may be negative or positive, because of the interdependencies between variables (Figure 2.5.1). Here, positive feedback is considered when the increase/decrease in the actual value of a variable, leads to an increase/decrease of another variable. The opposite effect is considered a negative feedback.

An example of a negative feedback is that an increase in $Q_{H}$ directly increases $\theta$ and the entrainment rate at MLH top. Higher $\theta$ reduces the gradient to surface skin temperature (lower $\Delta \theta$ ) and thus drives down $Q_{H}$ (Equation 2.4). The entrainment of air with higher $\theta$ could enhance $Q_{H}$, but the heating due to entrainment is primarily directed towards increasing MLH top rather than the near surface $\theta$. Moreover, the increase in $\theta$ will also increase $L W_{\text {down }}$, and thereby $Q^{*}$, and $Q_{E}$ as relative humidity is reduced and hence evaportranspiration is enhanced.

Another feedback mechanism also exists between the increase of MLH and the consequent entrainment of drier air in the mixed layer, which reduces $q_{a i r}$ and increases $Q_{E}$ (van Heerwaarden et al., 2009).

Moreover, any variation of a flux because of atmospheric feedbacks mechanisms can also affect the distribution of $Q^{*}$ to the other fluxes, thus creating an indirect effect. For 


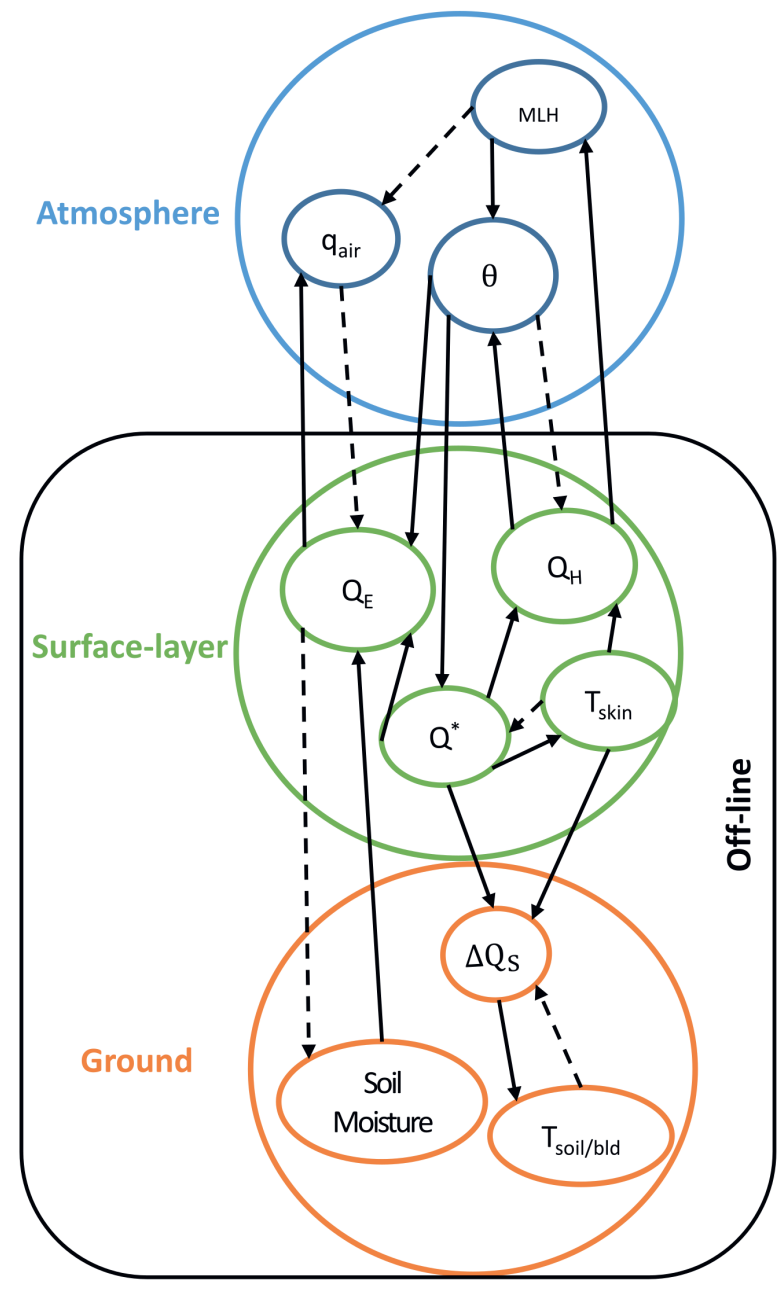

Figure 2.5.1: Conceptual relations of most important surface energy balance variables and their feedback mechanisms included in the on-line and off-line model set-ups. Surface variables (green) include $Q^{*}, Q_{H}, Q_{E}$ and skin temperature $\left(T_{\text {skin }}\right)$. Ground/Building variables (orange) include $\Delta Q_{S}$, soil moisture and soil/building temperature $\left(T_{\text {soil } / \text { bld }}\right)$. Atmospheric variables (blue) included mixed-layer height $(M L H)$, potential air temperature $(\theta)$ and atmospheric moisture $\left(q_{\text {air }}\right)$. Positive (solid arrows) and negative (dashed arrows) feedback mechanisms are indicated.

instance an increase in skin temperature of the urban area will decrease $Q^{*}$ due to higher $L W_{u p}$ and thus impact the turbulent heat fluxes as well. 


\subsection{Effects of surface parameters on the surface en- ergy balance}

\subsubsection{Urban fraction}

Sensitivity to urban fraction $\left(f_{\text {urb }}\right)$ impacts the total surface energy balance (Figure 2.6.1). The observed normalized daytime $Q^{*}$ is 0.403 (or $366 \mathrm{Wm}^{-2}$ ). The off-line value decreases from $0.364\left(329 \mathrm{Wm}^{-2}\right)$ to $0.346\left(313 \mathrm{Wm}^{-2}\right)$ as $f_{\text {urb }}$ increases. The on-line set-up decrease slightly faster from $0.378\left(348 \mathrm{Wm}^{-2}\right)$ to $0.351\left(324 \mathrm{Wm}^{-2}\right)$. The decrease of $Q^{*}$ with increasing urban fraction is caused by the increased short-wave absorption from the slightly lower urban albedo ( 0.15 vs 0.16 for vegetation) and from the increased $L W_{u p}$ in response to higher $T_{\text {skin }}$ (around $7.5 \mathrm{~K}$ off-line, $5.0 \mathrm{~K}$ on-line) for the urban compared to the vegetated surface.

The more rapid decrease in the on-line compared to the off-line normalized $Q^{*}$, is because of the faster increase in normalized $L W_{u p}$ (Figure A.1.1), a result of the increase in the on-line facet temperatures (by up to $1.2 \mathrm{~K}$ ) with increasing urban fraction. The increased on-line facet temperatures are caused by the faster increase in $\Delta Q_{S}$ in the on-line set-up, which is linked to the slower response of $Q_{H}$ because of the increase in $\theta$ (see feedbacks section 2.5 and Figure 2.5.1). In the off-line set-up the facet temperature does not vary with changing $f_{\text {urb }}$ since atmospheric $\theta$ does not vary. The same mechanism explains the deviations between on-line and off-line set-up in night-time $Q^{*}$.

Daytime normalized $Q_{H}$ (Figure 2.6.1), increases with increasing $f_{\text {urb }}$. The on-line normalized $Q_{H}$ ranges between $0.185-0.228$ (approximately a $39 \mathrm{Wm}^{-2}$ increase) while the off-line flux ranges between 0.167 to 0.221 ( $49 \mathrm{Wm}^{-2}$ increase). The increased $f_{\text {urb }}$ reduces evaporation (Figure 2.6.1g) and increases $\Delta Q_{S}$ (Figure 2.6.1e). The on-line $Q_{H}$ variation is $21 \%$ smaller than the off-line one. The slower increase in $Q_{H}$ is attributed to the faster decrease of $Q^{*}$ in the on-line set-up and the increase in near surface $\theta$ with increasing $f_{\text {urb }}$, which partially offset the increase of $Q_{H}$ due to higher skin temperature (as indicated above). This is confirmed by the slower increase in near surface air temperature gradient $(5.7 \mathrm{~K}$ to $6.7 \mathrm{~K})$ in the on-line set-up compared to the off-line one $(8.3 \mathrm{~K}$ to $11.0 \mathrm{~K})$. An increase in $Q_{H}$ with increasing $f_{\text {urb }}$ is also reported by the modelling study of Loridan et al. (2013) and the observation study of Kotthaus \& Grimmond (2014b). However, both also show that $Q_{H}$ is not only depended on $f_{u r b}$, but also on the difference in urban morphology found in the measurement source area in different wind directions, which make the response of $Q_{H}$ non-linear to the urban fraction.

Daytime $\Delta Q_{S}$ has a strong linear increase with $f_{\text {urb }} . \Delta Q_{S}$ ranges from $0.107\left(99 \mathrm{~W} \mathrm{~m}^{-2}\right)$ to $0.155\left(142 \mathrm{~W} \mathrm{~m}^{-2}\right)$ in the on-line case, while off-line ranges from $0.118\left(107 \mathrm{~W} \mathrm{~m}^{-2}\right)$ to $0.158\left(143 \mathrm{~W} \mathrm{~m}^{-2}\right)$. This value range is very similar to Loridan et al. (2013) findings for London. The increase in $\Delta Q_{S}$ is a direct response to the decrease in evaporation due 


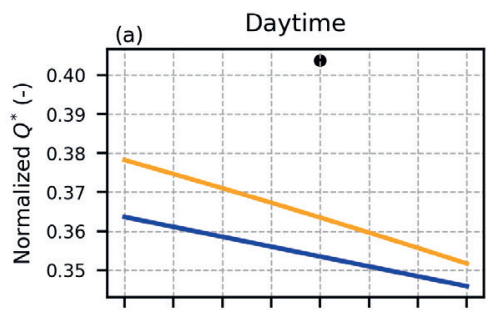

(c)
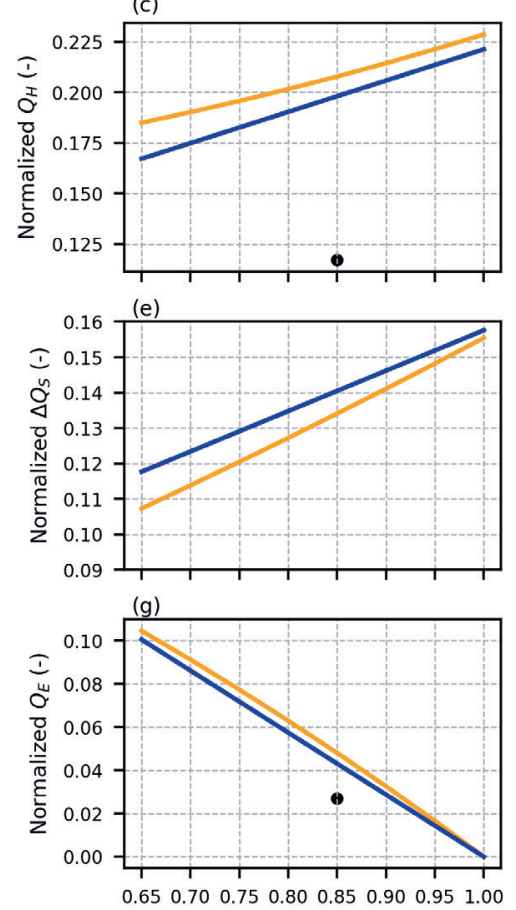

Urban fraction (-)
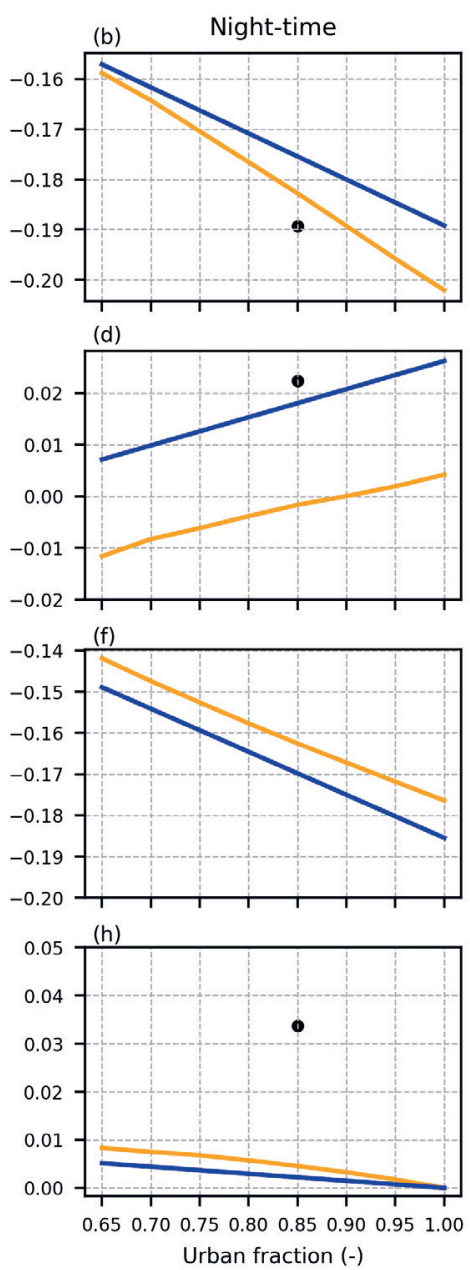

Urban fraction (-)

\section{- on-line $\quad$ off-line observations}

Figure 2.6.1: Normalized (a,b) $Q^{*},(\mathrm{c}, \mathrm{d}) Q_{H},(\mathrm{e}, \mathrm{f}) \Delta Q_{S}$ and (g,h) $Q_{E}$ for daytime (left) and night-time (right) as a function of the urban fraction for on-line (orange) and off-line (blue) cases. Modelled and observed fluxes are study period means (0600 UTC 23 July 2012 to 0600 UTC 25 July 2012).

to lower vegetation fraction. A small variation in the response of $\Delta Q_{S}$ exists between the two set-ups, indicating that the slower increase of skin to air temperature gradient in the on-line set-up is increasing the heat transfer rate into the urban fabric.

Finally, $Q_{E}$ decreases from $0.10\left(92 \mathrm{Wm}^{-2}\right)$ to 0 as vegetation fractions decrease, as 
the SLUCM scheme does not have integrated vegetation and because the green roof and anthropogenic latent heat options were both switched of for this experiment.

\subsubsection{Albedo}

Albedo governs the energy absorption of $S W_{\text {down }}$ and thus affecting $Q^{*}$ and all surface energy fluxes.

The $a_{\text {roof }}$ has the strongest impact on normalized daytime $Q^{*}$ (Figure 2.6.2a), which varies from 0.316 to 0.391 in the on-line case. That is effectively a difference of $69 \mathrm{~W} \mathrm{~m}^{-2}$ in the $Q^{*}$. The $a_{\text {wall }}$ impact is limited to $0.035\left(33 \mathrm{~W} \mathrm{~m}^{-2}\right)$ and the $a_{\text {road }}$ only to 0.011 (10 $\left.W m^{-2}\right)$. The importance of $a_{\text {roof }}$, over $a_{\text {wall }}$ and $a_{\text {road }}$, to the correct estimation of $Q^{*}$ is consistent with Loridan et al. (2010) and Zhao et al. (2014). Both indicate that the SLUCM urban morphology, makes the roof facet albedo critical for absorbed short-wave radiation, while the wall and road facet's are less important for $Q^{*}$. Difference in $Q^{*}$ response between on-line and off-line only occurs for the $a_{\text {roof }}$. This is a small increase (0.005 of the normalized $Q^{*}$ ) with higher albedo (Figure 2.6.2a).

Three mechanisms lie behind these differences in $Q^{*}$. As albedo increases, $L W_{\text {down }}$ decreases (up to $7 \mathrm{~W} \mathrm{~m}^{-2}$ ) due to lower air temperature and moisture content in the mixedlayer. On the other hand, $S W_{\text {down }}$ increases (up to $7 \mathrm{Wm}^{-2}$ ), because of the increased $S W_{u p}$, which is reflected by the atmosphere back towards the surface (Figure A.1.2a). This mechanism is included in the RRTMG short-wave scheme used. It is based on the two stream approach of Oreopoulos \& Barker (1999). This finding demonstrates the need to included atmospheric feedback in the model during sensitivity tests and also that multiple reflection radiation schemes (Iacono et al., 2008) should be selected over the simpler radiation schemes (Dudhia, 1989) during optimization of albedo values. On-line $L W_{u p}$ decreases faster than the off-line one with increasing albedo, because of faster decrease in facet's skin temperature in the on-line model (Figure A.1.2g). This feedback is linked to the decrease in near surface $\theta$ with increasing albedo.

Effectively only changes in $a_{\text {roof }}$ affect the on-line $Q_{H}$, with the normalized values varying from $0.229\left(211 \mathrm{~W} \mathrm{~m}^{-2}\right)$ to $0.173\left(159 \mathrm{~W} \mathrm{~m}^{-2}\right)$ (Figure 2.6.2c). The off-line simulation ranges from $0.225\left(204 \mathrm{~W} \mathrm{~m}^{-2}\right)$ to $0.153\left(138 \mathrm{~W} \mathrm{~m}^{-2}\right)$. Both have a strong linear response, as Zhao et al. (2014) found in their off-line SLUCM study. The decrease of $Q_{H}$ is primarily caused by the decrease of $Q^{*}$ as albedo increases. A difference of $0.016\left(15 \mathrm{~W} \mathrm{~m}^{-2}\right)$ in $Q_{H}$ response can be seen between the on-line and off-line model results for changing albedo. As explained in section 2.5, the negative feedback mechanism between $\theta$ and $Q_{H}$ (see Figure 2.5.1) limits the increase of on-line $Q_{H}$ caused by the albedo. Moreover, due to higher $Q_{H}$ there is more entrainment at the MLH top, which increases $\theta$, but most likely has a minimal effect on the near surface temperature since the heating from the entrainment is used to increase MLH. Furthermore, the increase in evaporation (Figure 2.6.2g), which is linked to atmospheric feedbacks, is a limiting factor for the increase of 

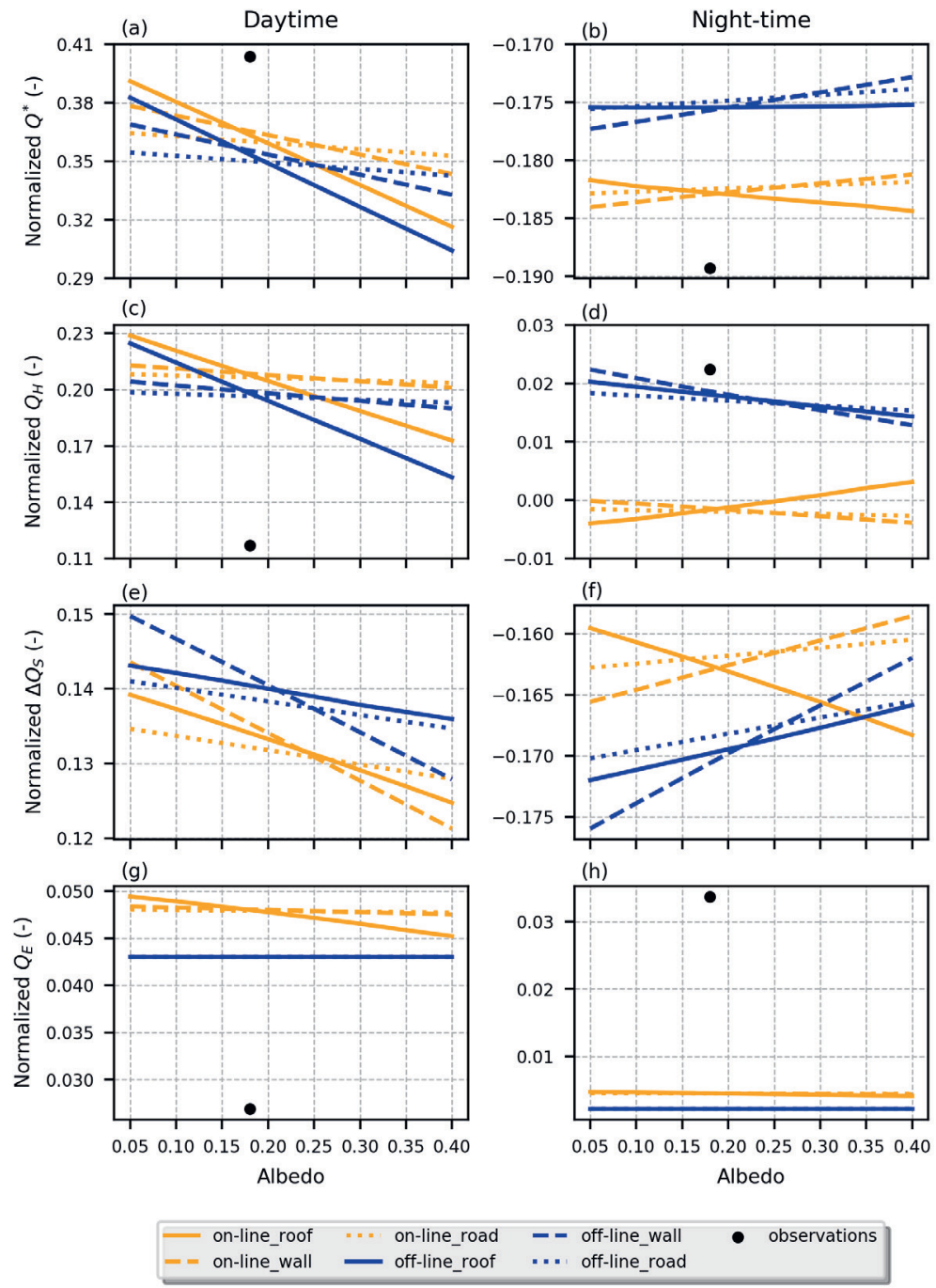

Figure 2.6.2: As in Figure 2.6.1, but for facets' albedo.

$Q_{H}$ in response to decreased albedo. These feedbacks, result in a $21 \%$ lower variation in $Q_{H}$ in the on-line case compared to the off-line. Night-time $Q_{H}$ has very small dependency on daytime albedo.

Variations in the modelled normalized $\left(\Delta Q_{S}\right)$ during daytime show a maximum range of $0.015\left(14 \mathrm{~W} \mathrm{~m}^{-2}\right)$ of the mean daytime downward radiation. Both cases show a similar sensitivity of $\Delta Q_{S}$ for wall and road albedo. However, between the off-line and on-line 
cases there is an increasing difference (up to 0.013) in the $\Delta Q_{S}$ flux for higher $a_{\text {roof }}$. The most likely explanation lies in the different energy partitioning between $Q_{H}$ and $Q_{E}$, which moderate the $\Delta Q_{S}$ flux (see Figure 2.5.1). Especially the decrease in evaporation could lead to higher $\Delta Q_{S}$ fluxes, an effect also suggested by Loridan et al. (2013). Roof and wall albedo are also contributing to variations of up to $0.010\left(3.5 \mathrm{~W} \mathrm{~m}^{-2}\right)$ in $\Delta Q_{S}$ during night-time. However, this difference is small compared to the mean night-time $\Delta Q_{S}$ values $\left(60-65 W m^{-2}\right)$.

Atmospheric feedbacks also influence the response of $Q_{E}$ flux (Figure 2.6.2g) to changes in facet's albedo. The off-line case has no variation in the $Q_{E}$, because the atmospheric forcing is fixed and the NOAH model calculates surface energy balance separately for nature and urban tiles. In the on-line case, the feedback mechanisms do alter the normalized $Q_{E}$ from the nature tile. Two atmospheric feedback mechanisms affect the evaporation are described in section 2.5. From their combined effect we find a decrease of $0.05\left(5 \mathrm{~W} \mathrm{~m}^{-2}\right)$ of the normalized mean daytime $Q_{E}$ for increasing albedo, nearly $11 \%$ of the mean daytime value. Thus, it is essential to include atmospheric feedbacks during evaluation of urban surface models, especially without integrated vegetation.

\subsubsection{Thermal conductivity}

The thermal conductivity changes the way energy is distributed and the amount of emitted $L W_{\text {up }}$.

Day and night-time $Q^{*}$ (Figure 2.6.3a,b) do not show any strong variation to changes in $\lambda_{\text {roof }}$ and $\lambda_{\text {road }}$. A noteworthy variation is the rate of change of $Q^{*}$ during night-time. It decreases sharply for low thermal conductivities $\left(0.15-0.45 \mathrm{~J} \mathrm{~K}^{-1} \mathrm{~m}^{-1} \mathrm{~s}^{-1}\right)$, while above $0.45 \mathrm{JK}^{-1} \mathrm{~s}^{-1}$ a saturation effect or even reversal of the slope (for the wall facet) occurs. The response of $Q^{*}$ is linked to the $L W_{u p}$ radiation from the surface and consequently the skin temperature of the facets. In both wall and roof facets the increase of conductivity up to $0.60 \mathrm{~J} \mathrm{~K}^{-1} \mathrm{~m}^{-1} \mathrm{~s}^{-1}$ results in more energy stored in the facets during daytime, which also increases their night-time skin temperature (by about $1 \mathrm{~K}$ ), resulting in an increased $L W_{u p}$ flux (Figure A.1.3h). For higher conductivity values the heat stored in the facet during daytime does not increase as fast, because it is limited by the heat capacity of the facet. Thus, the energy loss at night-time outweighs the energy gain during daytime for conductivity values above $0.60 \mathrm{~J} \mathrm{~K}^{-1} \mathrm{~m}^{-1} \mathrm{~s}^{-1}$, leading to a decrease in facets' temperatures.

The increase in thermal conductivity also increases normalized $\Delta Q_{S}$ (Figure 2.6.3e). Variations in $\Delta Q_{S}$, due to changes in lambda $a_{\text {roof }}$, range from $0.128\left(118 \mathrm{~W} \mathrm{~m}^{-2}\right)$ to 0.156 (143 $\left.W \mathrm{~m}^{-2}\right)$ for the on-line case, and $0.134\left(121 \mathrm{~W} \mathrm{~m}^{-2}\right)$ to $0.165\left(149 \mathrm{~W} \mathrm{~m}^{-2}\right)$ for the off-line one. At night variation in $\Delta Q_{S}$ ranges from $-0.154\left(-55 \mathrm{~W} \mathrm{~m}^{-2}\right)$ and $-0.160\left(-57 \mathrm{~W} \mathrm{~m}^{-2}\right)$ to $-0.171\left(-61 \mathrm{~W} \mathrm{~m}^{-2}\right)$ and $-0.177\left(-62 \mathrm{~W} \mathrm{~m}^{-2}\right)$, for on-line and off-line set-up respectively. A similar response is found for increasing $\lambda_{\text {wall }}$. Above $0.45 \mathrm{~J} \mathrm{~K}^{-1} \mathrm{~m}^{-1} \mathrm{~s}^{-1}$ normalized 

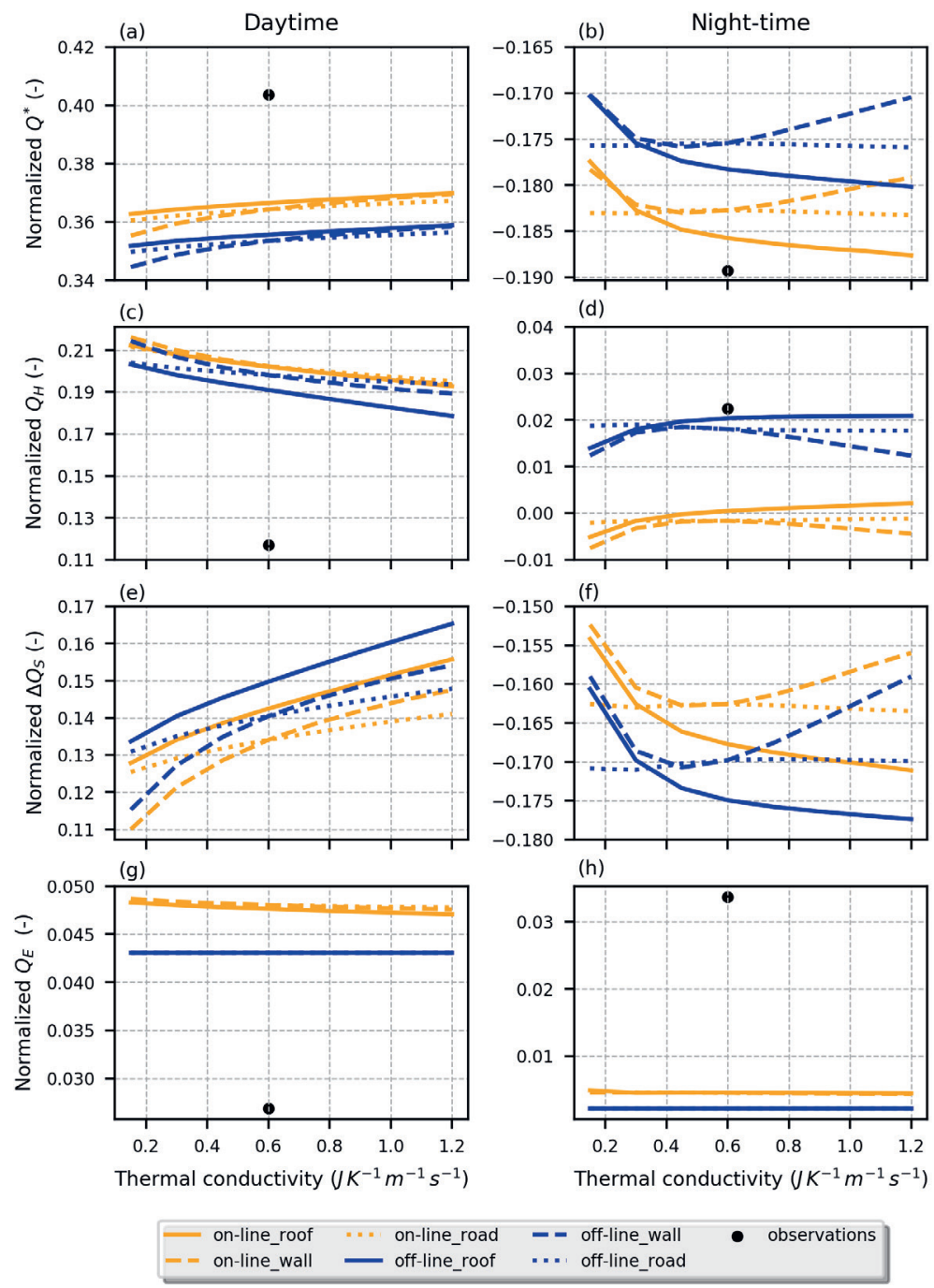

Figure 2.6.3: As in Figure 2.6.1, but for facets' thermal conductivity.

night-time $\Delta Q_{S}$ decreases, because the heat stored in the facet during daytime is not enough the compensate the loss during night-time (as explained above).

Normalized daytime $Q_{H}$ (Figure 2.6.3c) is decreasing by $0.019\left(17 \mathrm{~W} \mathrm{~m}^{-2}\right)$ for the on-line model (from 0.212 to 0.193 ) while for the off-line model the decrease is $0.025\left(23 W_{m}^{-2}\right)$. This decrease is linked to the increase in $\Delta Q_{S}$ (see Figure 2.6.3e), due to faster energy transmission to the urban fabric resulting in lower skin temperatures and thus smaller $Q_{H}$ 
flux (feedback in Figure 2.5.1). The difference in the response of normalized $Q_{H}$ between the two set-ups is $0.006\left(5 \mathrm{~W} \mathrm{~m}^{-2}\right)$ and is attributed to the smaller variation in skin to air temperature gradient in the on-line set-up compare to the off-line one.

\subsubsection{Heat capacity}

Much like thermal conductivity, heat capacity alters the amount of emitted $L W_{u p}$ and the energy partitioning at the surface.

The response of daytime normalized $Q^{*}$ to heat capacity of the facets is identical to those of the conductivity experiment. However, during night-time some changes occur. Instead of abrupt changes in $Q^{*}$, there is a more gradual change with increasing heat capacity (Figure 2.6.4b). For changes in $C_{\text {roof }}$, night-time $Q^{*}$ ranges from $-0.169\left(59 \mathrm{Wm}^{-2}\right)$ to $-0.195\left(-69 \mathrm{~W} \mathrm{~m}^{-2}\right)$ for the on-line case. A similar range of variation is seen for the offline model. The off-line case shows a higher $Q^{*}$, compared to the observed value of -0.19 $\left(67 \mathrm{~W} \mathrm{~m}^{-2}\right)$. The levelling of the $Q^{*}$ above $C_{\text {wall }}$ of $1.5 \mathrm{~J} \mathrm{~m}^{-3} \mathrm{~K}^{-1}$ is related to nearly constant $L W_{u p}$ flux (Figure A.1.4h), which indicates that there is no significant variation in facets temperatures increase past $1.5 \mathrm{~J} \mathrm{~m}^{-3} \mathrm{~K}^{-1}$, because the fixed value for $\lambda_{\text {wall }}$ is limiting the transfer of heat to (and from) the facet and thus reducing model sensitivity at higher $C_{\text {wall }}$. This interdependency between thermal conductivity and heat capacity also agrees with the conclusions of Loridan \& Grimmond (2012b) that optimization of surface parameters in UCMs requires accounting for combined effects when altering surface parameters values.

Normalized daytime $Q_{H}$ decreases is up to $0.015\left(14 \mathrm{Wm}^{-2}\right)$ due to changes in $C_{\text {roof }}$ and $C_{\text {wall }}$. The decrease of daytime $Q_{H}$ is caused by the high skin-to-building temperature gradient and $\Delta Q_{S}$ flux. The difference in the response of $Q_{H}$ to increasing heat capacity between the two set-ups is $0.004\left(4 \mathrm{~W} \mathrm{~m}^{-2}\right)$ and is linked to a faster decrease in skin to air temperature in the off-line compared to the on-line model, as heat capacity for roof increases. Night-time $Q_{H}$ shows the opposite response compared to the daytime $Q_{H}$ flux (Figure 2.6.4d). The on-line case shows a minimal $0.010\left(4 \mathrm{~W} \mathrm{~m}^{-2}\right)$ increase in night-time $Q_{H}$ with increasing $C_{\text {roof }}$ and a $0.016\left(6 \mathrm{~W} \mathrm{~m}^{-2}\right)$ increase for increasing $C_{\text {wall }}$. For the off-line set-up this increase is $0.018\left(6 \mathrm{~W} \mathrm{~m}^{-2}\right)$. The smaller variation in $Q_{H}$ flux for the on-line set-up is caused by the smaller variation of skin to air temperature difference.

Mean daytime $\Delta Q_{S}$ flux increases with increasing heat capacity for all facets, due to increased retention of heat. The variation in $\Delta Q_{S}$ for the on-line set-up is 0.020 (18 $\left.W \mathrm{~m}^{-2}\right)$ for change in $C_{\text {roof }}$ and $0.027\left(24 \mathrm{~W} \mathrm{~m}^{-2}\right)$ for changes in $C_{\text {wall }}$. For the off-line set-up the increase is $0.024\left(22 \mathrm{~W} \mathrm{~m}^{-2}\right)$ and $0.030\left(28 \mathrm{~W} \mathrm{~m}^{-2}\right)$, respectively. There is small increase in the difference between the two set-ups of around $0.004\left(4 \mathrm{~W} \mathrm{~m}^{-2}\right)$ at for higher heat capacities. Night-time $\Delta Q_{S}$ decreases as heat capacity increases, which is slower for the on-line set-up compared to the off-line set-up. The difference is 0.003 $\left(1 \mathrm{~W} \mathrm{~m}^{-2}\right.$ ) for low $C_{\text {roof }}$ value and $0.010\left(3 \mathrm{~W} \mathrm{~m}^{-2}\right)$ for high values. For the wall heat 

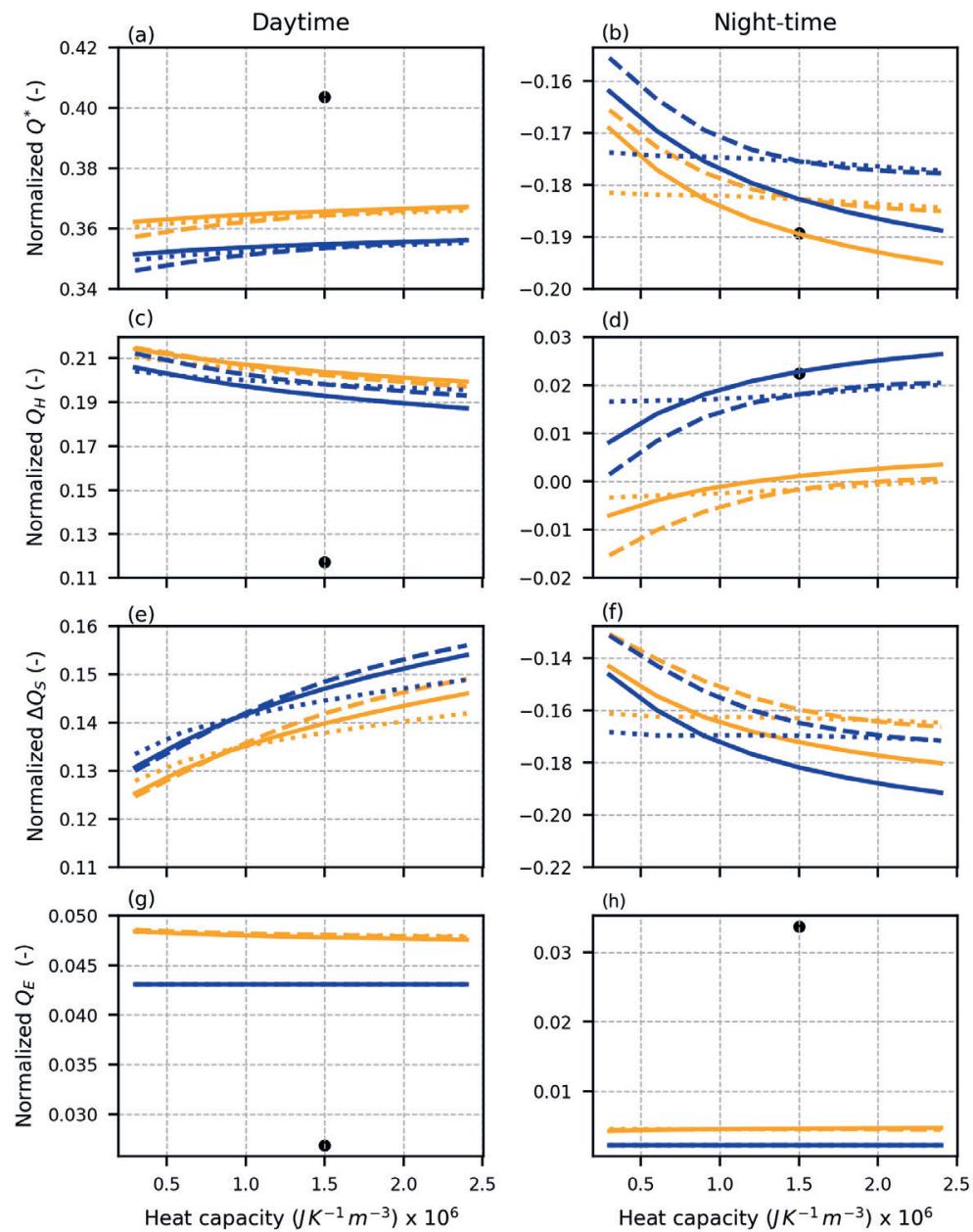

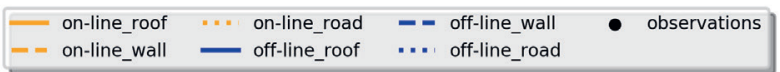

Figure 2.6.4: As in Figure 2.6.1, but for facets' heat capacity.

capacity the initial difference of $0.002\left(1 \mathrm{~W} \mathrm{~m}^{-2}\right)$ between the two set-ups reduces to 0.008 $\left(3 W m^{-2}\right)$.

\subsection{Discussion}

These results may differ from other urban surface schemes, because of both different model sensitivity and intensity of the feedback mechanisms. Thus, other schemes (simple "bulk" 
to more complex "multi-layer" urban canopy schemes) should be similarly studied in both on-line and off-line configurations.

Our on-line set-up used the MYNN boundary-layer scheme (Mikio \& Hiroshi, 2009), because it is well tested in combination with the MYJ surface layer scheme (Janjic, 1994). During evaluation it showed better results for neutral conditions compared to the MYJ boundary-layer scheme and also represented the night-time conditions better in comparison to the YSU scheme (Hong et al., 2006). For the on-line set-up the MYJ surface layer was also tested with the YSU boundary-layer scheme. Although results differ somewhat from the original on-line set-up, the feedback mechanisms are equally significant in altering the model's performance. However, given the differences in model sensitivity based on the boundary-layer scheme, further investigation of the interaction between boundary-layer schemes and the urban surface scheme would be useful.

Although both the off-line and on-line model use the same tiling approach to calculate the surface energy balance, we discovered that the net short-wave radiation for the vegetation tile is calculated different between the two set-ups. In the off-line model the net short-wave radiation for the vegetation tile is calculated using the albedo of the vegetation, while in the on-line the weighted average albedo (urban and vegetation) of the tile is used. This can lead to a deviation in the calculated $Q^{*}$ for the vegetation tile and consequently the $Q_{H}$ and $Q_{E}$ fluxes. To ensure that both set-ups calculate fluxes the same way we decided to use the off-line approach also in the on-line set-up, assuming that the vegetation is not influenced by variations in urban albedo.

The off-line model uses observations from one location (KSSW) as input, but for the on-line model runs multiple sources are required for the initial input and advection for temperature, moisture and momentum. While the effective forcing of the LSM and UCM are quite similar for both (see section 2.4), the differences may impact the behaviours of the urban surface scheme. Therefore a series of tests were performed to assess if the atmospheric forcing was not the main influence during the sensitivity tests. In the off-line model we repeated all facet's albedo sensitivity tests with forcing derived from the online model run with the default surface parameter configuration. Furthermore the on-line experiments for roof albedo were repeated without the advection of heat, moisture and momentum and compared the results with the default off-line experiment. In both cases we still observe differences in sensitivity response of the on-line and off-line set-ups.

The effects of facet emissivity on the surface energy balance were only minor (similar to Loridan et al., 2010). The main effect of changes in facet's emissivity is a slight increase in skin temperature of the facet and thus a slightly higher $L W_{u p}$ radiation from the surface. We did not find a direct increase in $L W_{u p}$ due to increase in the emissivity of the facets. The $L W_{u p}$ radiation calculated in the SLUCM is not transferred to the long-wave radiation scheme and is only used within the SLUCM scheme to calculate $Q^{*}$ for the urban patch. The radiation scheme instead calculates $L W_{u p}$ using the emissivity of the vegetated patch 
and the averaged urban and nature patch surface temperature. This results in a different $L W_{u p}$ radiation calculated by radiation scheme and the urban surface model, which only affects the atmosphere and not $Q^{*}$, because the latter uses the $L W_{u p}$ calculated from the urban scheme. In this study urban $L W_{u p}$ from both set-ups was calculated as in SLUCM.

Other parameters used by SLUCM could influence the model's performance. For instance, thickness of each facets layer and also the thickness distribution of the layers can affect the way heat is transferred to/from the urban fabric and thus affect the energy balance. Other parameters that do affect the model performance include: (a) empirical coefficient $a_{k}$ (used to calculated roughness length for heat over the canyon based on the roughness length of momentum Kanda et al., 2007), (b) anthropogenic heat and latent heat, (c) internal building temperature and others. Loridan et al. (2010) and Zhao et al. (2014) cover the sensitivity of most of the surface parameters for the off-line SLUCM model, but an on-line vs off-line comparison for these parameters would also be useful.

Here, clear sky conditions are chosen to minimize meteorological influence and maximises the impact of surface parameter changes. However, under other meteorological conditions (e.g. clouds or rain) model response may differ. For instance, during cloudy days with less short-wave radiation the predominant role of facets' albedo will be dampened, thus heat capacity of the urban fabric and the urban fraction may be more dominant factors. During rain and immediately following, water interception by the impervious surfaces alters evaporation and the surface energy balance (Grimmond \& Oke, 1991; Yang et al., 2016). As hydrological processes are implemented in SLUCM (Yang et al., 2015), the impact of changes to these parameters under varying weather conditions will be important to explore.

In this study we are concerned with the importance of the atmospheric feedbacks and their representation in a NWP model. Like all models, it will have incomplete representation of all the atmospheric feedbacks. For instance in a realistic scenario if the surface albedo of the urban area decreases, then the surface and air temperatures will increase, resulting in stronger gradients between the city and the rural areas. This can increase advection of colder air and counteract the effects of decreasing albedo. Similar atmospheric feedbacks are always present in the atmosphere and can be important components in estimating and understanding model sensitivities. However, they require a full 3D representation of the atmosphere to be explicitly resolved and studied.

\subsection{Conclusions}

The model behaviour of two Noah-SLUCM set-ups off-line and coupled to the atmosphere are investigated. After evaluation for a two-day summer period in London we varied a series of parameters, (a) urban fraction, (b) surface albedo, (c) thermal conductivity 
and d) thermal capacity of the urban facets, to assess the effects on the surface energy balance. We identified several differences in model response between the two set-ups, which we attributed to various feedback mechanisms between the surface and the overlying atmosphere.

The model evaluation revealed that the on-line set-up did well at capturing $Q^{*}, S W_{\text {down }}$ and $L W_{\text {down }}$, with small variations compared to the observations. Both on-line and offline models show large discrepancies for $Q_{H}$ and $Q_{E}$, due to shortcomings of the model and measurement uncertainties. Air temperature, wind speed and wind direction are relatively well represented in the on-line case. Boundary-layer height is well simulated on the first day, but is underestimated on the second day of the case study period.

A substantial sensitivity of $Q^{*}$ and turbulent fluxes to surface parameters is reported. During daytime, urban fraction and albedo are the primary contributors of variations to $Q^{*}, Q_{H}$ and $Q_{E}$, while heat capacity and thermal conductivity greatly affect the $\Delta Q_{S}$ flux. At night, urban fraction, heat capacity and thermal conductivity show stronger effects on $Q^{*}, Q_{H}$ and $\Delta Q_{S}$ flux, while variations in albedo have a small effect.

Finally, there are some distinct differences in the sensitivity of the on-line and the off-line set-ups, which have been attributed to feedback mechanisms between the surface and the atmosphere. Depending on the surface parameter, the effects of the atmospheric feedback mechanism can outweigh the variation due to the surface parameter change. Overall, $Q^{*}$ is not significantly affected by atmospheric feedback mechanisms. The effects are most profound for $Q_{E}$ and $\Delta Q_{S}$, where indirect atmospheric feedback can account for nearly $100 \%$ and $50 \%$, respectively. Feedback mechanisms also decrease the changes in daytime $Q_{H}$ by up to $22 \%$. Thus we recommend taking atmospheric feedback between surface and the atmosphere under consideration when evaluating the performance of urban canopy models for the aforementioned variables.

\section{Acknowledgements}

This research has been funded by the NWO grant no. 864.14.007. The authors would like to thank the ECMWF for providing operational analysis data, the University of Wyoming for the radio-sounding data and also NOAA for providing data for the WMO stations, all of which were used to create the forcing for the on-line model. We also would like to thank all the co-authors of the SUBLIME case study, which is utilised by this paper. 


\section{Chapter 3}

\section{Surface and atmospheric driven variability of the single-layer urban canopy model under clear sky conditions over London}

This chapter is based on:

Tsiringakis, A., Holtslag, A. A. M., Grimmond, S., \& Steeneveld, G. J. (2020). Surface and atmospheric driven variability of the single-layer urban canopy model under clear-sky conditions over London. Journal of Geophysical Research: Atmospheres, 125, e2019JD032167. https://doi.org/10.1029/2019JD032167 


\section{Abstract}

Urban canopy models (UCMs) are parametrization schemes that are used to improve weather forecasts in urban areas. The performance of UCMs depends on understanding potential uncertainty sources that can generally originate from the (a) urban surface parameters, (b) atmospheric forcing, and (c) physical description. Here, we investigate the relative importance of surface and atmospheric driven model sensitivities of the singlelayer urban canopy model when fully interactive with a 1-D configuration of the Weather Research and Forecasting model (WRF). The impact of different physical descriptions in UCMs and other key parameterization schemes of WRF is considered. As a case study, we use a 54-hr period with clear-sky conditions over London. Our analysis is focused on the surface radiation and energy flux partitioning and the intensity of turbulent mixing. The impact of changes in atmospheric forcing and surface parameter values on model performance appears to be comparable in magnitude. The advection of potential temperature, aerosol optical depth, exchange coefficient and roughness length for heat, surface albedo, and the anthropogenic heat flux are the most influential. Some atmospheric forcing variations have similar impact on the key physical processes as changes in surface parameters. Hence, error compensation may occur if one optimizes model performance using a single variable or combinations that have potential for carryover effects (e.g., temperature). Process diagrams help differences to be understood in the physical description of different UCMs, boundary layer, and radiation schemes and between the model and the observations. 


\subsection{Introduction}

Urban canopy models (UCM) are essential components of many numerical weather prediction (NWP) models as they represent sub-grid scale physical process of the urban fabric. However, given their complexity, UCMs' performance is not always well understood. Large variations in model performance have been reported between different UCM (Grimmond et al., 2010) with similar configurations for the urban surface parameters (Grimmond et al., 2010; Best \& Grimmond, 2015) and between the same UCM with different configurations (Loridan et al., 2010; Grimmond et al., 2010).

One source of uncertainty in the performance of a UCM originates from the complexity of the representation of the urban surface in the UCM (Best \& Grimmond, 2015). The complexity ranges from bulk schemes that only account for basic surface parameters, to multi-layer schemes accounting for building drag effects and street-canyon orientation (Masson, 2000; Kusaka et al., 2001; Martilli et al., 2002; Salamanca \& Martilli, 2009; Jarvi et al., 2011). The simpler physical description in less complex UCMs could potentially lead to worse performance from incomplete representation of physical mechanisms in the urban environment.

Best \& Grimmond (2015) showed that differences in complexity between UCM might not be the primary source of model bias. Their findings, supported also by those of Loridan et al. (2010), Demuzere et al. (2017) and Ronda et al. (2017) suggest that adequate prescription of urban surface parameters is equally essential (if not more) for reducing the biases between UCMs and observations. The surface parameters are also linked to key physical processes, like radiation absorption (e.g. surface albedo and emissivity) and surface energy partitioning. Given the plethora of parameters that are needed to be known, disentangling the contribution of each parameter on model performance can be time consuming (Loridan et al., 2010; Loridan \& Grimmond, 2012b; Zhao et al., 2014). An off-line multi-optimization, that minimizes errors due to uncertainties in surface parameters in a UCM is possible (Loridan et al., 2010). However, understanding the contribution of all surface parameter changes to key physical process in the urban fabric and the coupling with the overlying atmosphere is virtually an impossible task.

Complexity increases further when UCMs are coupled to the atmosphere. In such cases an off-line multi-optimization approach might not have the expected improvement in model performance. Thus, the model's response to changes in parameters setting varies substantially depending on whether a UCM is coupled with the atmosphere or not (Tsiringakis et al., 2019). However, when coupled to a NWP model, UCM uncertainty increases also from variations in atmospheric forcing provided by the different parameterisation schemes coupled with the UCM. Ferrero et al. (2018) found that BEP and BEP+BEM models to perform better when coupled to TKE-based boundary layer schemes. This highlights that the turbulent mixing intensity strongly affects model performance, through modification 
of near-surface atmospheric forcing. Such findings are also supported by Sterk et al. (2013) and Bosveld et al. (2014b).

Therefore, it is not surprising that in-depth knowledge on UCM performance when coupled to NWPs is still limited. It is essential to know which uncertainty sources could have the largest impact on the key radiative, surface energy and turbulent mixing processes. We investigate if different uncertainty sources have similar impacts on model variability to identify compensating effects on model bias. Changes in surface parameters and atmospheric forcing on three key physical process are tested. The results are utilized to identify why model performance varies between different UCMs coupled to the same NWP and between the same UCM coupled to different boundary layer and radiation schemes existing in the NWP model.

The impact of changes in surface parameters and atmospheric forcing on the surface radiation balance, surface flux partitioning and turbulent mixing in the single-layer urban canopy model (SLUCM, Kusaka et al. (2001)) coupled to the Weather Research and Forecasting (WRF) model (Skamarock et al., 2008; Powers et al., 2017) is studied using a case study (section 3.2). A brief evaluation with observations is conducted prior to an in-depth analysis of the model variability caused by changes in surface parameters and atmospheric forcing (section 3.3). We explore how physical processes modified by changes in the atmospheric forcing and surface parameters can explain variations between different parameterisation schemes (section 3.4). Finally discussion and conclusions are drawn (sections 3.5 and 3.6).

\subsection{Methodology}

\subsubsection{Case Study Description}

A clear-sky 54-hour period (00:00 UTC 23 July 2012 to 05:00 UTC 25 July 2012) from the SUBLIME study (Steeneveld et al., 2017; Tsiringakis et al., 2019) is used. A spin-up time of 6 hours is used to allow building temperatures adjust to the changes in atmospheric forcing components and surface parameters. The first 24-hours (06:00 UTC 23 July 2012 to 05:00 UTC 24 July 2012) are used for the primary focus of this paper. (sections 3.3.2, 3.3.3, 3.3.4 and 3.4). For the following 24-hours (06:00 UTC 24 July 2012 to 05:00 UTC 25 July 2012) the sensitivity analyses are repeated. This latter material and comparison to the prior day is presented in the supporting information.

Observations taken at the King's College London measurement site (KSSW) (Kotthaus \& Grimmond, 2014a,b), include temperature, wind, radiation and surface fluxes at 50 $\mathrm{m}$ above ground level (a.g.l.). The local urban surface properties used for the reference experiment (Table 3.2.1) are based on existing literature for the KSSW site (Kotthaus \& Grimmond, 2014a,b) and central London (Bohnenstengel et al., 2011; Oikonomou et al., 
2012). Other surface parameters used (not in Table 3.2.1) are : a) average building height, b) aspect ratio, c) roof width, d) albedo, e) heat capacity, f) thermal conductivity and g) emissivity values for all facets. These are from SUBLIME (Tsiringakis et al., 2019).

Table 3.2.1: Experiment with reference values held constant when another parameter is varied across the uncertainty range. Naming uses the surface parameters tested and its value. See Table A.2.1 for notation definitions.

\begin{tabular}{cccc} 
Experiment name & Parameter & Reference & Values used \\
\hline$f_{\text {urb_value }}$ & $f_{\text {urb }}(0-1)$ & 0.85 & $0.75,0.80,0.85,0.90,0.95$ \\
$\alpha_{\text {roof_value }}$ & $\alpha_{\text {roof }}(0-1)$ & 0.18 & $0.10,0.15,0.18,0.25,0.30$ \\
$\lambda_{\text {wall_value }}$ & $\lambda_{\text {wall }}\left(\mathrm{J} \mathrm{s}^{-1} \mathrm{~K}^{-1}\right)$ & 0.60 & $0.15,0.45,0.60,0.75,1.05$ \\
$C_{\text {wall_value }}$ & $C_{\text {wall }}\left(\mathrm{J} \mathrm{m}^{-3} \mathrm{~K}^{-1} * 10^{6}\right)$ & 1.50 & $0.60,1.20,1.50,1.80,2.40$ \\
$a_{\text {kanda_value }}$ & $a_{\text {kanda }}(-)$ & 1.00 & $0.50,0.80,1.00,1.10,1.40$ \\
$Q_{f \_v a l u e}$ & $Q_{f}\left(\mathrm{Wm}^{-2}\right)$ & 38.0 & $10.0,30.0,38.0,50.00,70.0$ \\
\hline
\end{tabular}

The atmospheric forcing (Steeneveld et al., 2017; Tsiringakis et al., 2019) includes initial profiles for potential temperature, wind, mixing ratio (up to $17 \mathrm{~km}$ ) and surface pressure. The 1D-WRF model initialization profiles are based on a radio-sounding (UWYO, 2012) at Herstmonceux, Hailsham, UK at 00UTC 23 July 2012 and are adjusted using the KSSW measurements and 3D WRF model derived profiles over London. The adjustment with KSSW and 3D WRF is done to ensure an accurate estimate of boundary temperature, moisture and wind given the $70 \mathrm{~km}$ seperation between KSSW and Herstmonceux.

Boundary conditions for the 1D-WRF model are applied in the form of subsidence, geostrophic wind and advection tendency terms for potential temperature, moisture, $\mathrm{u}$ and v wind-components. Geostrophic wind is derived from 6-hourly ECMWF operational reanalysis data (ECMWF, 2012) in combination with a 3D WRF simulations (hourly data)(Steeneveld et al., 2017; Tsiringakis et al., 2019). Geostrophic wind values are given as 6-hourly means, with a tendency term applied to the $\mathrm{u}$ and $\mathrm{v}$ geostrophic wind components at each time step (of $1 \mathrm{D}-\mathrm{WRF}$ ) to ensure a smooth change in geostrophic wind through the 6-hourly blocks and avoid oscillations from imbalance between actual and geostrophic wind speeds.

Advection for potential temperature, mixing ratio and momentum ( $\mathrm{u}$ and $\mathrm{v}$ wind) are imposed throughout the 30-hour period as additional tendency terms in the prognostic equations for the specified variables. These tendency terms are derived from 3D WRF model simulations for London (Tsiringakis et al., 2019). The hourly advection terms from 3D WRF are averaged with advection estimates from WMO stations (NOAA/NCDC, 2012) within and around London. Six-hourly means are obtained from the hourly values. Thus a static advection is prescribed (i.e. independent of the 1D-WRF temperatures) that changes every 6 hours in the 1D-WRF simulation. Advection is treated as uniform throughout the observed height of the boundary layer, with a sharp linear decrease above. 
This new approach is preferred to avoid daytime stable stratification from a sharp decrease of negative temperature advection between $500-1500 \mathrm{~m}$, as in the original SUBLIME forcing (Steeneveld et al., 2017; Tsiringakis et al., 2019). The latter affects the distribution of TKE in the upper part of the boundary layer, thus impacting the boundary layer height and the temperature and moisture profiles.

Initial soil temperature and moisture content profiles (to $1.5 \mathrm{~m}$ depth), surface temperature for vegetation and urban surfaces are provided. They are derived from a 3D WRF simulation and then cycled $3 \times 2$ days in an off-line setup, until the deepest soil temperature became constant and storage heat flux shows a similar diurnal range for both days of the case study (Tsiringakis et al., 2019).

\subsubsection{Model description and setup}

Here we use the single-column version of WRF v3.8.1 Skamarock et al. (2008). The urban surface is parameterized based on the SLUCM scheme (Kusaka et al., 2001; Chen et al., 2011a) with the Noah-LSM scheme (Chen \& Dudhia, 2001) representing the vegetated land-surface processes. SLUCM separates the urban surface into three facets (roof, road and wall), each with their distinct sky-view factor based on urban morphological parameters. The turbulent sensible heat $\left(Q_{H}\right)$ flux from each facet is calculated:

$$
Q_{H}=\rho c_{P} C_{H} U_{a}(\Delta \theta),
$$

where $\rho$ is the air density $\left(\mathrm{kg} \mathrm{m}^{-3}\right), c_{p}$ is the specific heat capacity of dry air $\left(\mathrm{J} \mathrm{kg}^{-1} \mathrm{~K}^{-1}\right)$ and $C_{H}$ is the exchange coefficients for heat. $\Delta \theta(K)$ is the potential temperature difference between the surface and the air. $U_{a}$ is the wind speed $\left(\mathrm{m} \mathrm{s}^{-1}\right)$ at the first model level. The urban and the vegetation fluxes are combined using a tiling approach based on their plan area fraction. The anthropogenic heat flux $\left(Q_{f}\right)$ (added to the first model level) is prescribed with a diurnal cycle. For more details about SLUCM physical description and parameters see Loridan et al. (2010).

The surface layer and boundary layer are parameterized using Mellor-Yamada-Janjic (MYJ) schemes (Janjic, 1994). Radiative processes for long-wave and short-wave radiation are obtained from the RRTMG radiation schemes (Iacono et al., 2008), while for microphysics the WSM 3-class order scheme (Hong et al., 2004) is used. The model uses 70 vertical levels that extent to $17 \mathrm{~km}$, with 25 levels within the lowest $1.5 \mathrm{~km}$.

\subsubsection{Strategy for the sensitivity tests}

Sensitivity tests are conducted to address the three sources of uncertainty influencing model performance: a) surface parameters, b) atmospheric forcing and c) the differences in physical description in other essential parameterisation schemes. The aim is to identify the effects these have on the surface radiation, energy fluxes and turbulent intensity. 


\section{Surface parameters}

Based on Loridan et al. (2010) and Zhao et al. (2014), we identify urban fraction $\left(f_{\text {urban }}\right)$, albedo $(a)$, thermal conductivity $(\lambda)$, heat capacity $(C)$, the $a_{\text {kanda }}$ parameter and the anthropogenic heat flux $\left(Q_{f}\right)$ as the most influential surface parameters. Note as $Q_{f}$ is prescribed in SLUCM it is treated as a parameter rather than a variable. For simplicity we investigate only the impact of roof albedo $\left(a_{\text {roof }}\right)$, wall thermal conductivity $\left(\lambda_{\text {wall }}\right)$ and heat capacity $\left(C_{\text {wall }}\right)$ rather than all facets. Tsiringakis et al. (2019) found these to have the dominant impact on model performance in the current model configuration.

The $a_{\text {kanda }}$ parameter, modifies the roughness length of heat $\left(Z o_{h c}\right)$ from the one of momentum $\left(Z o_{m c}\right)$, above the urban canyon and the overlying atmosphere (Kanda et al., 2007; Loridan et al., 2010) via,

$$
Z o_{h c}=Z o_{m c} e^{\left(2-a_{\text {kanda }}\left(R e_{c}^{*}\right)^{0.25}\right)},
$$

where $R e_{c}^{*}$ is the Reynolds's roughness number. Hence the $a_{k a n d a}$ primarily influences the ratio between sensible $\left(Q_{H}\right)$ and storage $\left(\Delta Q_{s}\right)$ heat fluxes, with larger values decreasing $Q_{H}$ (and vice versa). It thus impacts the skin temperatures, surface flux partitioning, turbulent mixing in the surface layer, and the outgoing long-wave radiation $\left(L W_{u p}\right)$ due to lower $\Delta Q_{s}$.

$Q_{f}$ is a very uncertain parameter as in reality it is highly variable with strong dependence on anthropogenic activities (Iamarino et al., 2012; Bohnenstengel et al., 2014; Dong et al., 2017). For London values can vary between 10-140 $\mathrm{Wm}^{-2}$, depending on the location and area extent, with estimates reaching $200 \mathrm{Wm}^{-2}$ in central London (Iamarino et al., 2012), but small when averaged over larger areas (Lindberg et al., 2013; Dong et al., 2017). Ward et al. (2016) estimates $Q_{f}$ for this study area to vary temporally between 20 and $80 \mathrm{Wm}^{-2}$. Considering how important the anthropogenic heat flux can be for the surface energy balance (Best \& Grimmond, 2016) it is essential to include it in our analysis. Reference values, incremental change and minimum/maximum variation limits for the surface parameters (Table 3.2.1) are based on Loridan et al. (2010); Iamarino et al. (2012); Zhao et al. (2014); Ward et al. (2016); Dong et al. (2017) and Tsiringakis et al. (2019).

\section{Atmospheric forcing}

The atmospheric effects investigated are the impact of radiation, advection of heat and moisture and turbulent mixing intensity. Short-wave radiation biases in cities are common even in clear sky conditions (Tsiringakis et al., 2019) by WRF, especially due to aerosol loading effects on direct short-wave radiation (Gomes et al., 2008; Kokkonen et al., 2019). Hence we modulate the aerosol optical depth (AOD) to simulate effects on radiation between clearer and more polluted atmospheric conditions. Terra/MODIS satellite derived AOD (Levy et al., 2013) range between 0.1 and 0.4 (0.18 in the reference run) depending 
on the distance from central London and the timing of the measurement (NASA/EOSDIS, 2019). Long-wave radiation biases in models participated in GABLS (including WRF) (Kleczek et al., 2014; Bosveld et al., 2014b) suggest that long-wave downward radiation $\left(L W_{\text {down }}\right)$ biases are caused by bias in boundary layer temperature and humidity and different physical complexity of the radiation models. Using an extreme $\mathrm{CO}_{2}$ uncertainty range $(38-3800 \mathrm{ppm})$ we increase and decrease the long-wave downward $\left(L W_{\text {down }}\right)$ radiation without changing directly air temperature or humidity. This allows us to further disentangle the atmospheric driven uncertainty in $L W_{\text {down }}$ from the physical driven one.

Heat $\left(A D V_{\theta}\right)$ and moisture $\left(A D V_{q}\right)$ advection are inherently difficult to estimate correctly especially in heterogeneous environments. Yet they strongly impact the near surface temperature and the surface energy balance (Heaviside et al., 2015). $A D V_{\theta}$ and $A D V_{q}$ forcing are both positive and negative in the study period. Multiplication factors (Table 3.2.2) are applied to the negative advection values. Positive advection values receive the inverse multiplication factor for these tests (e.g. when $A D V_{\theta}<0$ values are multiplied by 1.25 , positive values are multiplied by 0.8 and vice versa).

Table 3.2.2: As Table 3.2.1, but for atmospheric forcing. Aerosol optical depth (AOD) and $\mathrm{CO}_{2}$ values change, whereas the remainder use a multiplication factor.

\begin{tabular}{|c|c|c|c|}
\hline Experiment & Atmospheric forcing & Reference & Values used \\
\hline AOD_value & $A O D(0-1)$ & 0.18 & $0.00,0.09,0.18,0.27,0.36$ \\
\hline $\mathrm{CO}_{2 \_ \text {value }}$ & $\mathrm{CO}_{2}(\mathrm{ppm})$ & 380 & $38,76,380,1900,3800$ \\
\hline$C H_{\text {heat_value }}$ & $C H_{\text {heat }}$ (mult. factor) & 1.00 & $0.67,0.80,1.00,1.25,1.50$ \\
\hline $\mathrm{CH}_{\text {mom_value }}$ & $C H_{\text {mom }}$ (mult. factor) & 1.00 & $0.67,0.80,1$ \\
\hline$A D V_{\theta-v a l u e}$ & $A D V_{\theta}$ (mult. factor) & 1.00 & $0.67,0.80,1.00,1.25,1.50$ \\
\hline$A D V_{q-v a l u e}$ & $A D V_{q}$ (mult. factor) & 1.00 & $0.67,0.80,1.00,1.25,1.50$ \\
\hline
\end{tabular}

To investigate the impact of turbulent mixing intensity we modify the exchange coefficients for heat $\left(\mathrm{CH}_{\text {heat }}\right)$ and momentum $\left(\mathrm{CH}_{\text {mom }}\right)$ for the urban, surface-layer and boundarylayer schemes. These coefficients are linearly (Table 3.2.2) enhanced or decreased to modify the coupling between the surface and overlying atmosphere. The turbulent mixing impacts the performance of the surface-layer scheme (Sterk et al., 2013; Bosveld et al., $2014 \mathrm{~b}$ ) and can explain much of the biases (i.e long-wave radiation, near surface temperature and surface energy fluxes) between model results and observations. Here we investigate fully convective boundary layers, whereas previously neutral and stable boundary layers have been studied. Hence our range of the multiplication factors for the exchange coefficients (0.67-1.50) is smaller than that used by Sterk et al. (2013) and Bosveld et al. (2014b) (0.25-4). 


\section{Physical ensemble tests}

Variability in model performance is also caused by the model physics used to parameterize sub-grid scale processes. The PILPS urban ((Grimmond et al., 2010; Grimmond et al., 2011)) and the GABLS ((Bosveld et al., 2014b; Kleczek et al., 2014)) studies have investigated this variability in model performance for different UCMs, boundary layer and surface-layer schemes. Using different NWP models (as seen in GABLS) complicates the analysis of model differences, as physics schemes also vary, thus adding uncertainty.

Using WRF, we can vary the individual physics schemes, while keeping others unchanged giving us a "physical ensemble" that enables us to test the third source of uncertainty (physics description). Here we consider the radiative transfer, boundary layer and UCM schemes available in WRF (Table 3.2.3).

Table 3.2.3: Parameterization schemes varied from the reference setup in seven runs $(R)$. Acronyms are explained in Appendix A.2.1

\begin{tabular}{ccccc} 
Run & SW Radiation & LW radiation & boundary layer & UCM \\
\hline R1(reference) & RRTMG & RRTMG & MYJ & SLUCM \\
R2 & CAM & - & - & - \\
R3 & - & CAM & - & - \\
R4 & - & - & YSU & - \\
R5 & - & - & QNSE & - \\
R6 & - & - & - & BEP \\
R7 & - & - & - & BEP+BEM
\end{tabular}

For the urban surface, BEP and BEP+BEM multi-layer urban canopy models are tested (Martilli et al., 2002; Salamanca \& Martilli, 2009) with SLUCM (Kusaka et al., 2001) being the reference choice. For the boundary layer we test the Yonsei University scheme (YSU) (Hong et al., 2006) and Quasi-normal Scale Elimination (QNSE) Scheme (Sukoriansky et al., 2005) both coupled to WRF-Noah-SLUCM setup. The radiation schemes is changed to the CAM short-wave and long-wave radiation schemes (Collins et al., 2004).

\subsubsection{Analysis of process diagrams}

Process diagrams (Sterk et al., 2013; Bosveld et al., 2014b) allow the impact of atmospheric forcing, surface parameters or parameterization schemes changes to be explored relative to a control run for a pair of variables. Each model run is represented by the mean of the two variables under investigation for a specified period (e.g. day-time or night-time). We link these points to identify if the response is linear or non-linear. Model sensitivity tests allows identification of the dominant influences on the pair of variables under investigation, within the ranges of the perturbed parameters and atmospheric forcing components (Tables 3.2.1, 3.2.2, 3.2.3). The mean observations are shown to help 
explain differences between model and observations. Following Bosveld et al. (2014b) we use four perturbations and the reference model run to capture changes in parameters and atmospheric forcing components (including drawing the sensitivity lines), but we only show the maximum and minimum limits as points in the figures.

Sterk et al. (2013) and Bosveld et al. (2014b) suggest it is essential to identify variables combinations that are coupled/interdependent and are linked to the physical processes under investigation. Given the observations available we focus on a) surface radiation balance, b) surface energy flux partitioning and c) turbulent mixing separated by the time of day (based on surface net-radiation $\left.Q^{*}\right)$ into day $\left(Q^{*}>0 W \mathrm{~m}^{-2}\right)$ and night $\left(Q^{*}\right.$ $<0 W \mathrm{~m}^{-2}$ ) (Best \& Grimmond, 2015), allowing the analysis of results under strong and weaker turbulent mixing regimes, when different physical processes dominate.

\subsection{Results}

\subsubsection{Model evaluation for the reference model setup}

Here we briefly evaluate the $50 \mathrm{~m}$ air temperature and net all-wave radiation $\left(Q^{*}\right)$. For a more extensive evaluation see Tsiringakis et al. (2019).

For the entire evaluation period (06:00 UTC 23 - 06:00UTC 24 July) the reference run (R1) has a mean bias error $(M B E)$ of $0.11^{\circ} \mathrm{C}$ (Figure 3.3.1). The modeled $T_{50 m}$ is cooler during daytime $\left(M B E=-0.55^{\circ} \mathrm{C}\right)$ and warmer at night $\left(M B E=0.90^{\circ} \mathrm{C}\right)$ compared to the observations, suggesting the simulated temperature range is important. Therefore, the changes in temperature $\left(\Delta T_{50 \mathrm{~m}}=T_{50 \mathrm{~m}, \max }-T_{50 \mathrm{~m}, \min }\right)$ during daytime and night-time is also considered. The observed daytime $\Delta T_{50 \mathrm{~m}}$ is $9.85^{\circ} \mathrm{C}$ and $9.52^{\circ} \mathrm{C}$ when modeled (Figure 3.3.1, 3.3.3). A night-time $M B E$ of $0.75^{\circ} \mathrm{C}$ exists between the modeled $\Delta T_{50 m}$ $\left(6.26^{\circ} \mathrm{C}\right)$ and observed $\left(7.01^{\circ} \mathrm{C}\right)$.

For $Q^{*}$ the reference run model has an $M B E$ of $-23.3 \mathrm{Wm}^{-2}$ originating from -43.6 $W m^{-2}$ day $M B E$ and a $0.7 W^{-2}$ night. The daytime bias originates from shortwave downward radiation $S W_{\text {down }}\left(\mathrm{MBE}=14.3 \mathrm{Wm}^{-2}\right)$, shortwave upward radiation $S W_{u p}($ $\left.M B E=24.8 \mathrm{Wm}^{-2}\right)$, long-wave downward radiation $L W_{\text {down }}\left(M B E=-16.1 \mathrm{Wm}^{-2}\right)$ and long-wave upward radiation $L W_{u p}\left(M B E=20.9 \mathrm{Wm}^{-2}\right)$ (Figure A.3.1) biases. The nocturnal bias in $Q^{*}$ originates from $L W_{\text {down }}$ and $L W_{u p}$ biases of -8.1 and $-8.8 W^{-2}$, respectively.

\subsubsection{Surface Radiation Balance}

From the sensitivity analyses the bias in $S W_{\text {down }}$ is caused by aerosol optical depth (AOD), as all other parameter changes do not decrease $S W_{\text {down }}$. An AOD of 0.27 reduces the bias in $S W_{\text {down }}$. Terra/MODIS AOD data (Levy et al., 2013) for the study period (not shown) indicate a sharp increase of AOD from 0.15 (outskirts of London) to 0.25 (central 

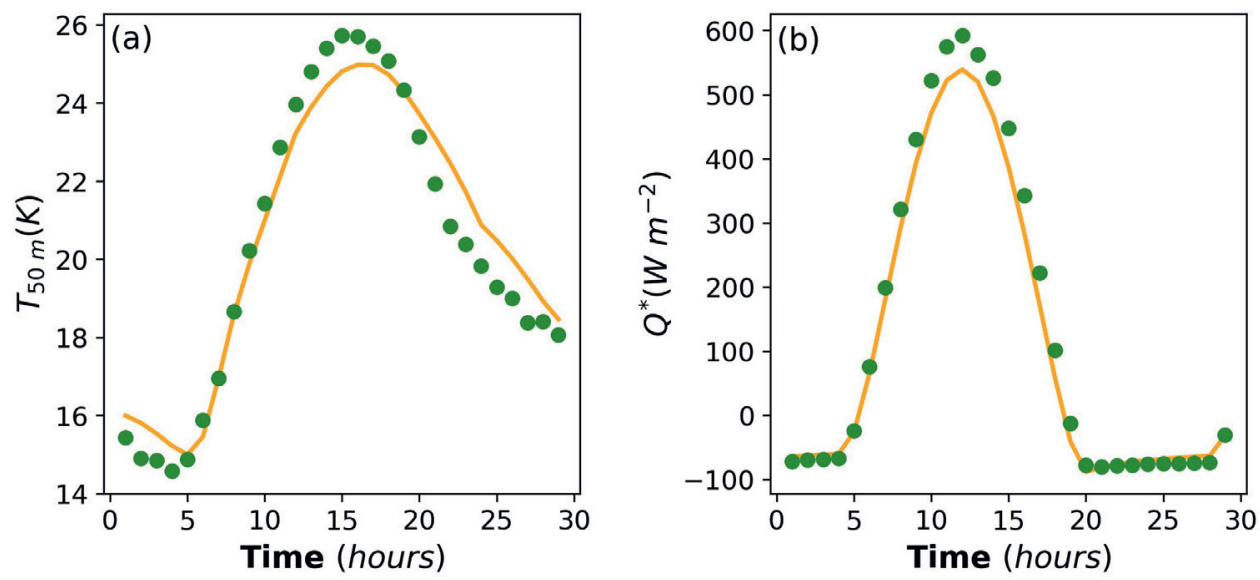

WRF-SLUCM-Noah

- observations

Figure 3.3.1: Modeled (reference setup) and observed hourly (a) air temperature $\left({ }^{\circ} \mathrm{C}\right.$ ) and (b) net all-wave radiation $\left(Q^{*}\right)\left(W m^{-2}\right)$ at $50 \mathrm{~m}$ above ground level at the KSSW site for the period 01:00 UTC 23 July 2012 to 06:00 UTC 24 July 2012.

business district, CBD). However, improving the $S W_{\text {down }}$ estimate increases $Q^{*} \mathrm{MBE}$ (to $\left.-52 \mathrm{Wm}^{-2}\right)$ and net short-wave $\left(S^{*}\right)$ at the surface. As the bias originates primarily from $S W_{u p}$, we can attribute this to the bulk albedo of the urban surface. By decreasing $a_{\text {roof }}$ to 0.12 we decrease the surface albedo (Figure $3.3 .2 \mathrm{~b}$ ) and reduce the $Q^{*} \mathrm{MBE}$ (to 40 $W m^{-2}$ ). The bias in bulk albedo originates from the 2-D canyon physical description in SLUCM (Tsiringakis et al., 2019).

The remaining bias in $Q^{*}$ is from the net long-wave radiation $\left(L^{*}\right)$. The daytime bias in the $L W_{\text {down }}$ is only partially explained by uncertainty in $A D V_{\theta}$ and $A D V_{q}\left(\sim 4-5 \mathrm{Wm}^{-2}\right.$, Table 3.3.1a). Large changes in $\mathrm{CO}_{2}$, not supported by observational data, would be sufficient to account for this bias. However, it is more likely that the bias originates from a negative bias in mid-to-upper boundary layer temperature and moisture, but this cannot be verified with the existing observations. The $L W_{u p}$ biases are attributed to four different sources $\left(\mathrm{CH}_{\text {heat }}, a_{\text {kanda }}, c_{\text {wall }}\right.$ and $\left.\lambda_{\text {wall }}\right)$, with the first two being the stronger contributors. $C H_{\text {heat }}$ and $a_{\text {kanda }}$ alter $Q^{*}$ by modulating the $L W_{u p}$ radiation via changes in skin temperature, with larger values for $\mathrm{CH}_{\text {heat }}$ (and lower for $a_{\text {kanda }}$ ) removing heat faster from the surface and thus reducing skin temperatures and $L W_{u p}$.

While correcting the bias in $Q^{*}$ is arbitrary, it does not lead to better model performance in general. The response of $\Delta T_{50 m}$ for a given change in $Q^{*}$ strongly depends on which atmospheric forcing or surface parameter is modified (Figure 3.3.3). 

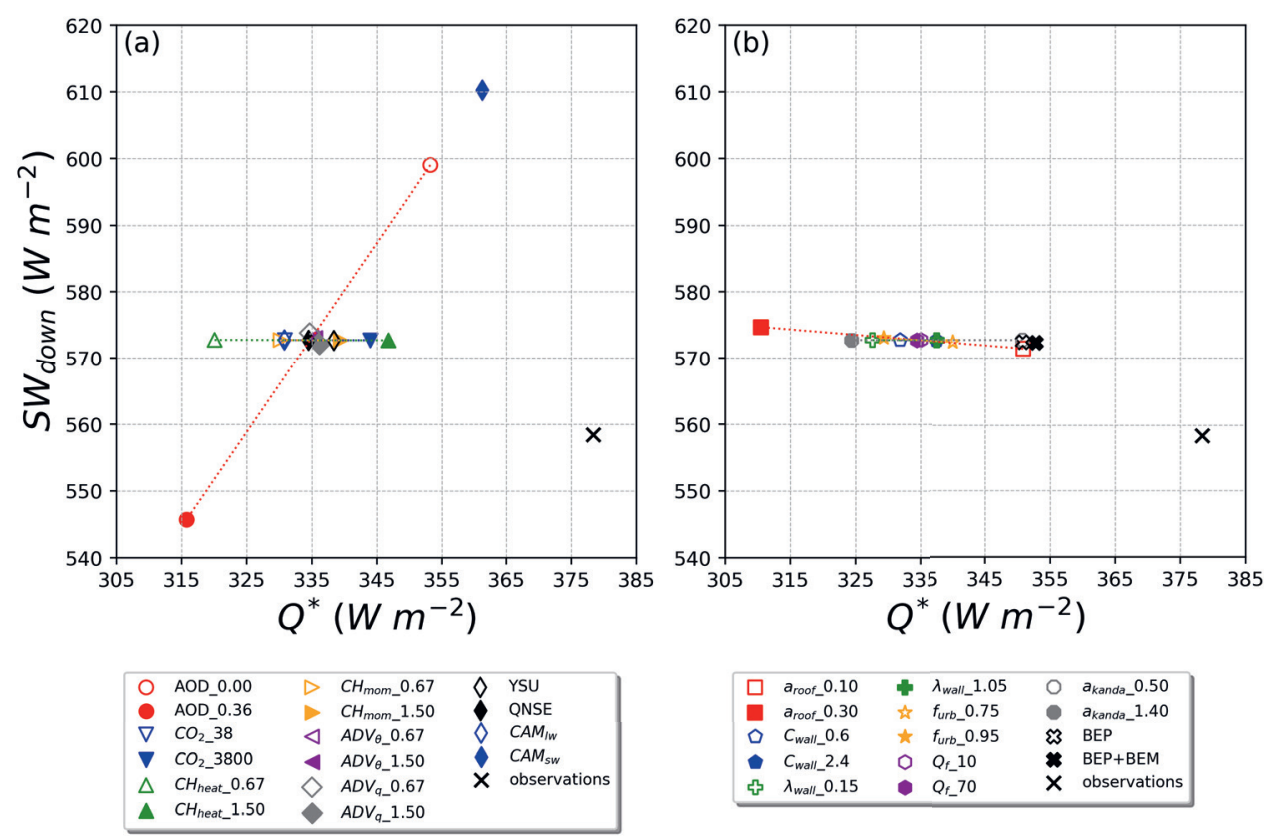

Figure 3.3.2: Sensitivity analysis across modeled uncertainty ranges (Tables 3.2.1, 3.2.2, 3.2.3). Impact on the average $S W_{\text {down }}$ and $Q^{*}$ with changes in (a) atmospheric forcing, boundary layer and radiation schemes and (b) changes in surface parameters and UCMs for daytime (06:00 UTC 23 July 2012 - 18:00 UTC 23 July 2012). For surface parameter (Table 3.2.1) and atmospheric forcing components (Table 3.2.2) only model runs using minimum (open symbol) and maximum (filled symbol) limit of the uncertainty range are shown, with lines between through all five runs.

During the day, changes in $A D V_{\theta}$ have small effect in $Q^{*}$, but strongly impact $\Delta T_{50 m}$ (1.8 $\mathrm{K}$ difference). The lack of variability in $Q^{*}$ is from compensating changes in $L W_{\text {down }}$ and $L W_{u p}$ (Table 3.3.1a) indicating that the boundary layer is in radiative balance with the surface, despite the drastic temperature change. An opposite effect is observed for changes in AOD, where large increases in $Q^{*}$ (up to $-37 \mathrm{Wm}^{-2}$ from 0 to 0.36 AOD) are not followed by a changing in $\Delta T_{50 m}$. This is caused by the increase in radiative heating due to $S W_{\text {down }}$ absorption at higher aerosol concentrations, which compensates for the decrease of air temperature by decreasing $Q_{H}$. Changes in $C H_{\text {heat }}$ and $C H_{\text {mom }}$ have positive $Q^{*}-\Delta T_{50 m}$ relations with strong radiative heating at higher $Q^{*}$ and vice versa (Figure 3.3.3a,b, A.3.7a and A.3.8a). The change in $\Delta T_{50 m}$ can be attributed to the changes in $Q_{H}$, which are caused by the changes in $C H_{\text {heat }}$ (equation 3.2).

Variations in SLUCM surface parameters have different impacts. Changes in $a_{\text {roof }}$ and $a_{\text {kanda }}$, like $\mathrm{CH}_{\text {heat }}$ and $\mathrm{CH}_{\text {mom }}$, have a positive trend (Figure 3.3.3b, A.3.3a, A.3.7a, 
Table 3.3.1: Difference $(\delta)$ in average $Q^{*}, S W_{\text {down }}, L W_{\text {down }}, S W_{\text {up }}, L W_{\text {up }}, \Delta T_{50 m}, \Delta T_{\text {skin }}$ and $q_{50 \mathrm{~m}}$ for a change between the maximum and minimum limits (Table 3.2.1 and 3.2.2) for the atmospheric forcing and the urban surface parameters for

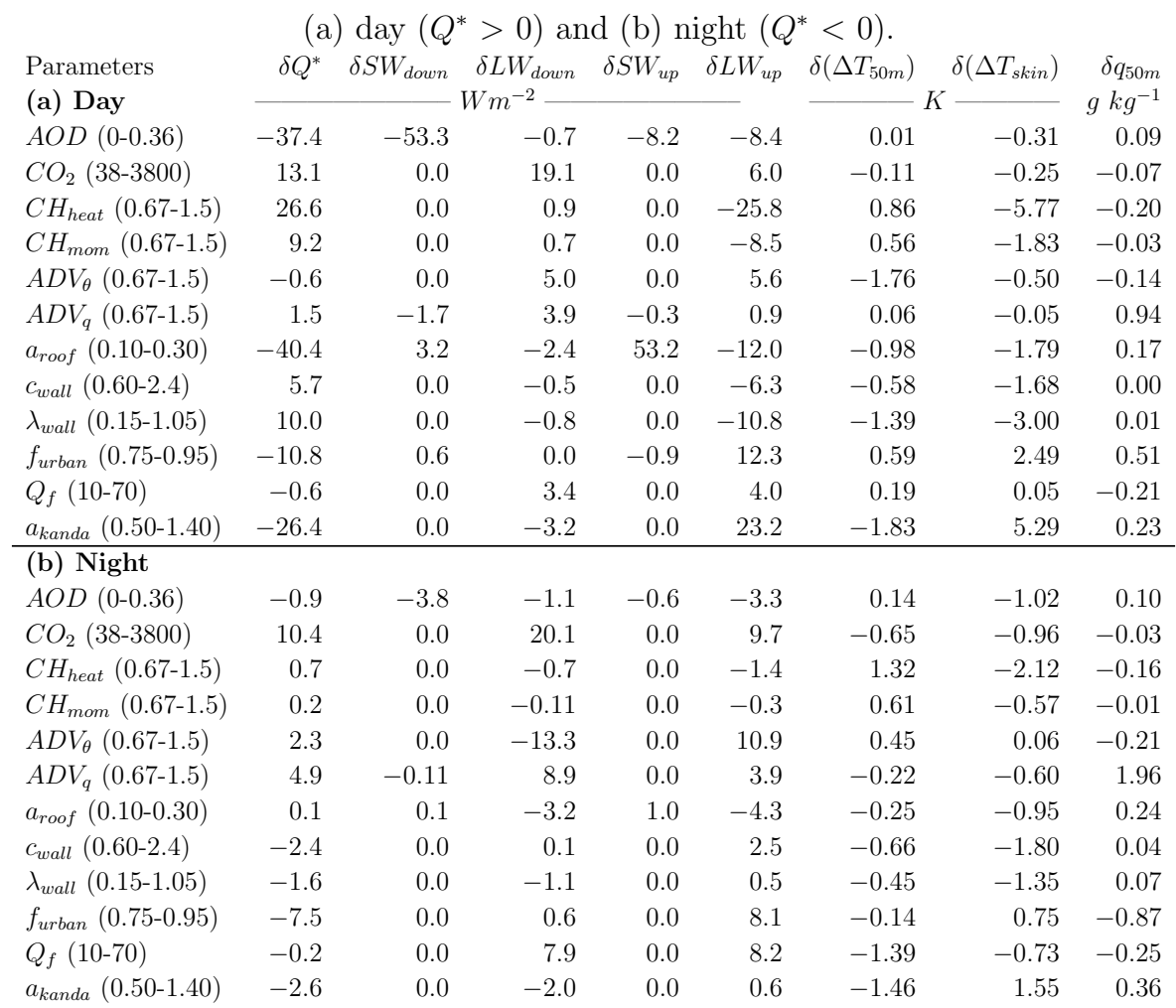

A.3.8a), with $a_{\text {roof }}$ having relatively larger impact on $Q^{*}$ (up to $37.4 \mathrm{Wm}^{-2}$ ), whereas $a_{\text {kanda }}$ has more impact on $\Delta T_{50 m}$. The $a_{\text {roof }}$ and $a_{\text {kanda }}$ increase $Q^{*}$ (and consequently $Q_{H}$ ), thus increasing $\Delta T_{50 m}$ as well. However the effect of $a_{\text {kanda }}$ on $Q_{H}$ is also from changes in the roughness length of heat (equation 3.1), which also impact the exchange coefficient of heat (equation 3.2).

The effects of changing $f_{\text {urban }}, C_{\text {wall }}$ and $\lambda_{\text {wall }}$ causes an increase in $\Delta T_{50 m}$ with a decrease in $Q^{*}$ (Figure 3.3.3b). $L W_{\text {up }}$ increases with smaller $C_{\text {wall }}, \lambda_{\text {wall }}$ and larger $f_{\text {urban }}$ values (Table 3.3.1a). $\Delta T_{50 m}$ increases with decreasing $C_{\text {wall }}$ and $\lambda_{\text {wall }}$ as $\Delta Q_{s}$ is decreased, hence increasing $Q_{H}$, while increasing $f_{\text {urban }}$ changes the vegetation fraction, thus decreasing the $Q_{E}$ and consequently increasing $Q_{H}$. Changes in $Q_{f}$ and $A D V_{\theta}$ both have the same orientation (Figure 3.3.3a,b) but different magnitude, with $Q_{f}$ not really affecting $\Delta T_{50 m}$ during the daytime.

At night changes in $A D V_{\theta}, A D V_{q}$ and $C_{2}$ impact both $Q^{*}$ and $\Delta T_{50 m}$ (Figure 3.3.3c). 

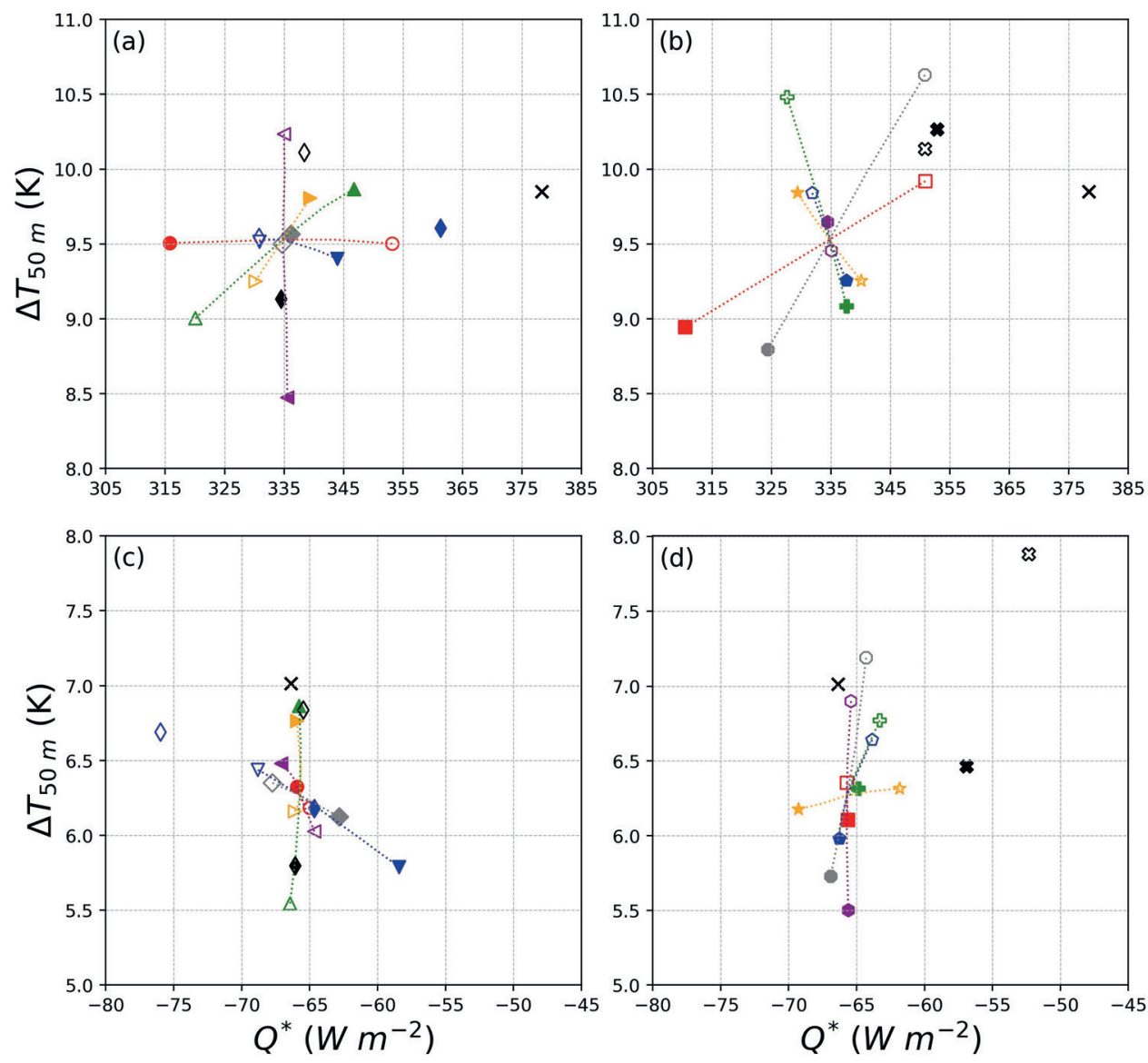

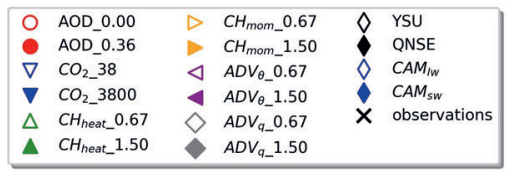

\begin{tabular}{|c|c|c|c|c|c|}
\hline$\square$ & $a_{\text {roof_}} 0.10$ & 4 & $\lambda_{\text {wall_}} 1.05$ & 0 & $a_{\text {kanda__ }} 0.50$ \\
\hline & aroof_ 0.30 & से & $f_{u r b_{-}} 0.75$ & - & $a_{\text {kanda_}} 1.40$ \\
\hline 0 & $C_{\text {wall_}} 0.6$ & $\star$ & $f_{\text {urb__ }} 0.95$ & $\mathfrak{\xi}$ & BEP \\
\hline - & $C_{\text {wall_}} 2.4$ & 0 & $Q_{f_{-}} 10$ & $\boldsymbol{*}$ & $\mathrm{BEP}+\mathrm{BEM}$ \\
\hline क & $\lambda_{\text {wall_}} 0.15$ & 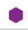 & $Q_{f_{-}} 70$ & $x$ & observations \\
\hline
\end{tabular}

Figure 3.3.3: As Figure 2, but for the impact on change in $50 \mathrm{~m}$ air temperature $\left(\Delta T_{50 \mathrm{~m}}\right.$ $\left.=T_{50 m, \max }-T_{50 m, \min }\right)$ and $Q^{*}$ with changes in (a,c) atmospheric forcing, boundary layer and radiation schemes and $(\mathrm{b}, \mathrm{d})$ in surface parameters and UCMs during $(\mathrm{a}, \mathrm{b})$ daytime (06:00 UTC 23 July 2012 - 18:00 UTC 23 July 2012) and (c,d) night-time (19:00 UTC 23 July 2012 - 05:00 UTC 24 July 2012).

However, changes in $A D V_{\theta}$ influence $\Delta T_{50 m}$ more, while $A D V_{q}$ mostly affects $Q^{*}$, by increasing $L W_{\text {down }}$ radiation due to more water vapor in the boundary layer. The prescribed $\mathrm{CO}_{2}$ concentration changes results in substantial changes in nocturnal $Q^{*}\left(>10 \mathrm{Wm}^{-2}\right.$ ) and $\Delta T_{50 m}$. Increasing $C H_{\text {heat }}$ and $C H_{\text {mom }}$ result in an increase in $\Delta T_{50 m}$ at night, which 
counteracts the daytime $\mathrm{CH}_{\text {heat }}$ effects (Tables 3.3.1a,b and Figure A.3.8). The increase in night-time $\Delta T_{50 m}$ is due to a decrease in daytime $\Delta Q_{s}$ (Figure 3.3.4a), which leads to smaller release of heat during the night (Figure 3.3.5a) and thus more radiative cooling. Consequently nocturnal air temperatures are lower in experiments with high $C H_{\text {heat }}$ (Figure A.3.8).

Changes in $Q_{f}$ and $a_{\text {kanda }}$ strongly influence nocturnal $\Delta T_{50 m}$ (similar to $C H_{\text {heat }}$ ), but cause minimal variation in $Q^{*}$, given the radiative balance between $L W_{u p}$ and $L W_{\text {down }}$ (Figure 3.3.3c and Table 3.3.1b). Increasing $a_{\text {kanda }}$ leads to a small decrease in nocturnal $Q^{*}$ due to the decrease in $L W_{\text {down }}$, a result of the lower air temperature due to less $Q_{H}$ during the day. $C_{\text {wall }}$ and $\lambda_{\text {wall }}$ have a non-linear behavior at night-time, because of heat saturation effects of the urban fabric (Tsiringakis et al. (2019) and section 3.3.3). Both have a large impact on nocturnal $\Delta T_{50 \mathrm{~m}}$, but also small effect (up to $3 \mathrm{Wm}^{-2}$ ) on $Q^{*}$. Increasing $f_{\text {urban }}$ results in the same decrease in $Q^{*}$ from higher $L W_{u p}$, but only a minor decrease in $\Delta T_{50 \mathrm{~m}}$. This suggests that night-time cooling rates surprisingly do not show a strong response to changes in $f_{\text {urban }}$. This can be explained by the strength of nocturnal radiative cooling. As discussed appendix A.3, the increase in mean daytime air temperature due to higher $f_{\text {urban }}$ leads to similar (or even stronger) cooling during the night (Figures A.3.6d, A.3.8a,b). This non-linear feedback involves an increase in day-time $T_{50 m}$ followed by an increase in night-time surface to air temperature gradient, thus leading to stronger atmospheric stability and more radiative cooling (Figures A.3.710 ). Changes in $f_{\text {urban }}$ are not the only triggering mechanism. It exists for most surface parameters and atmospheric forcing components that we investigated. Its impact is largest with lower wind speeds during the second day of the case study (appendix A.3).

For the radiative balance and its effects on radiative heating and cooling, we find five sources of uncertainty in this case study accounting for a large part of the bias between model and observations. As discussed, correcting AOD and $a_{\text {roof }}$ can explain the bias net-short-wave radiation and some of the bias in daytime $\Delta T_{50 m}$. Decreasing $a_{k a n d a}$ and increasing $\mathrm{CH}_{\text {heat }}$ effectively removes heat faster from the urban surface, thus decreasing skin temperature and $L W_{u p}$ flux, while increasing $\Delta T_{50 m}$ in both day and night. Finally, a small increasing in the intensity of $A D V_{\theta}$ can compensate the $\Delta T_{50 m}$ during the day. Some bias still remains in $Q^{*}$ and is primarily associated with the bias in $L W_{\text {down }}$ and some remaining bias in $L W_{u p}$.

\subsubsection{Energy partitioning}

Analysis of the surface energy partitioning is essential to understand the overall impact on atmospheric forcing and surface parameter changes in $\Delta T_{50}$. It provides further insight in compensating effects between day and night.

Regarding changes in atmospheric forcing during the daytime, AOD and $\mathrm{CO}_{2}$ effectively maintain the same energy partitioning ratio (1.55 to 1.59$)$ for both positive and negative 
changes in $Q^{*}$. Consequently any energy gain or loss at the surface is distributed equally between the $Q_{H}$ and $\Delta Q_{s}$ fluxes (Figure 3.3.3a). Smaller $C H_{\text {heat }}$ and $C H_{\text {momentum }}$ impose a variation in energy partitioning, with lower values leading to faster decrease in $Q_{H}$ and increase in $\Delta Q_{s}$. The variation here is primarily caused by the response of $Q_{H}$ due to changes in $\mathrm{CH}_{\text {heat }}$ (equation 3.2). $Q_{E}$ also decreases, but the flux variation from changes in $C H_{\text {heat }}$ is an order of magnitude smaller than for $Q_{H}\left(5\right.$ vs $40 \mathrm{Wm}^{-2}$ ). The decrease in $Q_{H}$ results in less heat directed towards the atmosphere and more heat stored in the urban fabric, thus decreasing $Q^{*}$ because of lower $L W_{\text {down }}$ and larger $L W_{u p}$ (Table 3.3.1a). Changing advection have similar effects, but with lower variation in energy partitioning and no effect on $Q^{*}$ due to the net long-wave radiative compensation.

Surface parameter changes have a wider impact in the parameter space (Figure 3.3.4b) compared to the change in atmospheric forcing, for the current choice of uncertainty range. Decreasing $a_{\text {roof }}$ increases the $Q_{H} / \Delta Q_{s}$ flux ratio, with faster increase in $Q_{H}$ compared to $\Delta Q_{s}$ due to thermal saturation effect on the roof facet. Thus, for the same change in $Q^{*}$ there is a larger variability in $\Delta T_{50 m}$ compared to changes in AOD (Figure 3.3.3b). Changes in $a_{\text {kanda }}$ have a nearly identical response as $C H_{\text {heat }}$ for the same reasons. The same occurs for changes in $Q_{f}$ and $A D V_{\theta}$, but despite their similar response in $Q^{*}$ and energy partitioning, each parameter affects the $\Delta T_{50 m}$ differently during the day and night. $C_{\text {wall }}$ and $\lambda_{\text {wall }}$ increase the $Q_{H} / \Delta Q_{s}$ ratio with decreasing parameter values due to higher skin temperature and higher $L W_{u p}$.

Although some atmospheric forcing $\left(\mathrm{CH}_{\text {heat }}\right.$ and $\left.\mathrm{CH}_{\text {mom }}\right)$ and surface parameters $\left(\mathrm{C}_{\text {wall }}\right.$ and $\left.\lambda_{\text {wall }}\right)$ cause a large variation in flux ratio and $\Delta T_{50 m}$, their impact on $T_{50 m}$ is small. This can be explained by the opposite effects on $T_{50 m}$ between night and day (Figure A.3.8). For instance an increase in $C H_{\text {heat }}$ leads to an increase in $Q_{H}$ during the day, but also decrease $Q_{H}$ at night-time (Figure 3.3.4a,c). Figure 3.3.5 shows that heat supply from the urban fabric at night is lower due to less $\Delta Q_{s}$ stored during the day as a result of the increase in $\mathrm{CH}_{\text {heat }}$. These compensating effects are what limits the change in $T_{50 m}$. Whereas, parameters like $Q_{f}$ and $A D V_{\theta}$ have smaller variations in the $Q_{H} / \Delta Q_{s}$ flux ratio, but have strong impact on $\Delta T_{50 m}$ both during day $\left(A D V_{\theta}\right)$ or night $\left(Q_{f}\right)$. Hence, both affect the day and night-time $T_{50 m}$ due to strong effects on their temperature that propagate from day to night and vice versa (Figure A.3.8, A.3.9).

Following Tsiringakis et al. (2019) we do not derive the storage heat as the residual of surface energy balance, because of the accumulation of errors (Grimmond \& Oke, 1999) and mismatch between the measurement footprint of the turbulent fluxes and the radiation fluxes (Schmid et al., 1991). Thus, we do not derive observed flux ratio during the day. However, the nocturnal $Q_{H} / \Delta Q_{s}$ ratio are strongly impacted by the sign and value of $Q_{H}$, given the difference between modeled $\left(0.15 \mathrm{Wm}^{-2}\right)$ and observed $(8.74$ $\left.W m^{-2}\right)$ nocturnal $Q_{H}$, and the plausible range of nocturnal $\Delta Q_{s}\left(-50\right.$ to $\left.-100 \mathrm{Wm}^{-2}\right)$. At night-time we use the objective hysteresis model (Grimmond \& Oke, 1991) with the 

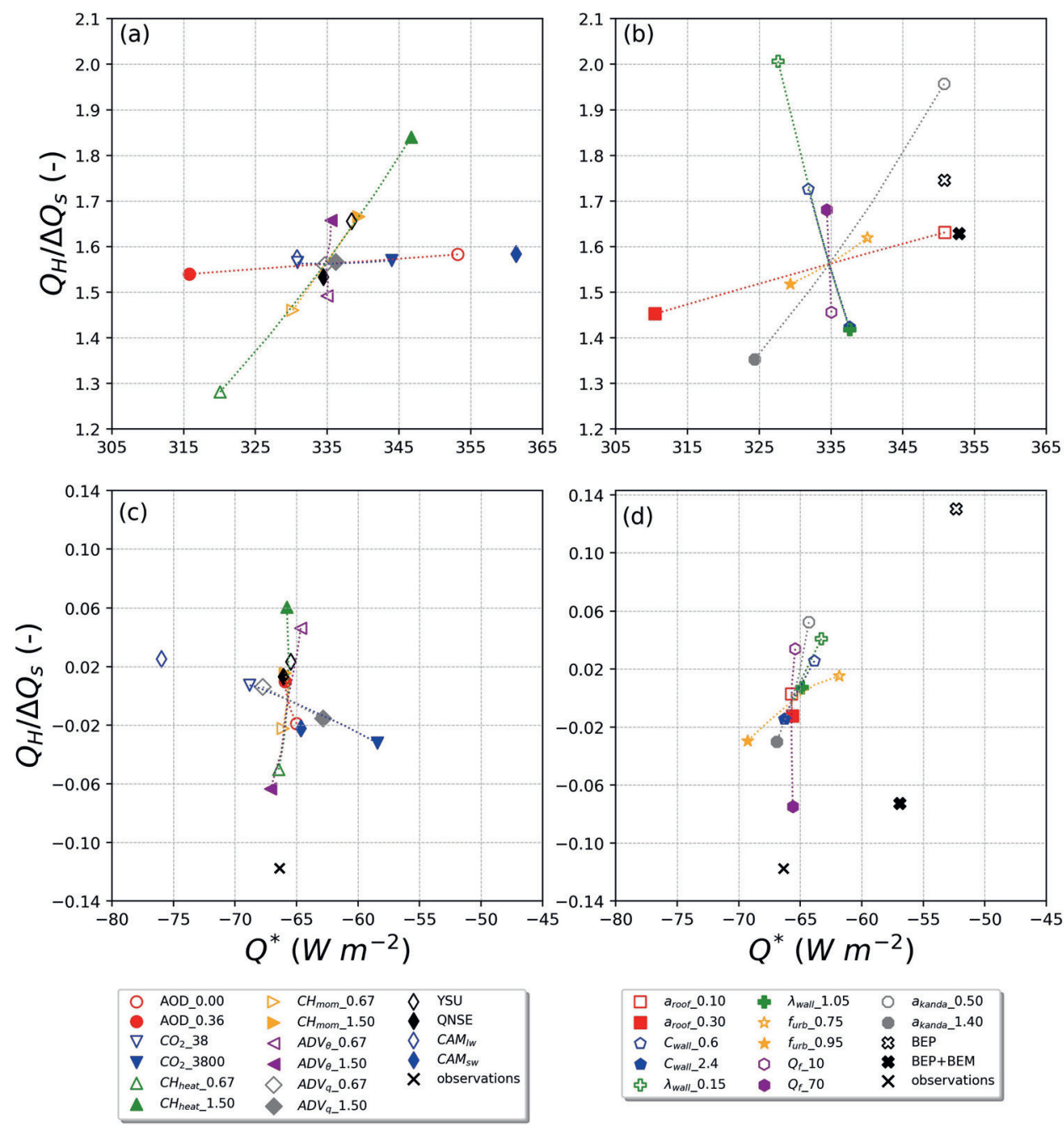

Figure 3.3.4: As Figure 3.3.3, but for the average surface flux ratio $\left(Q_{H} / \Delta Q_{s}\right)$ and net radiation $Q^{*}$.

same coefficients as (Ward et al., 2016) for this site forced by the observed $Q^{*}$ to derive an "observed" nocturnal $\Delta Q_{s}\left(-74.3 \mathrm{Wm}^{-2}\right)$, leading to an "observed" $Q_{H} / \Delta Q_{s}$ estimate of -0.12. Although this is not an observation it allows exploration of how atmospheric forcing or surface parameters might explain the bias between model and observations.

At night the $Q^{*}$ bias between model and observations is small $\left(3 \mathrm{Wm}^{-2}\right)$, but the flux ratio is much smaller in the model because $Q_{H}$ is nearly zero at night in the reference run. 

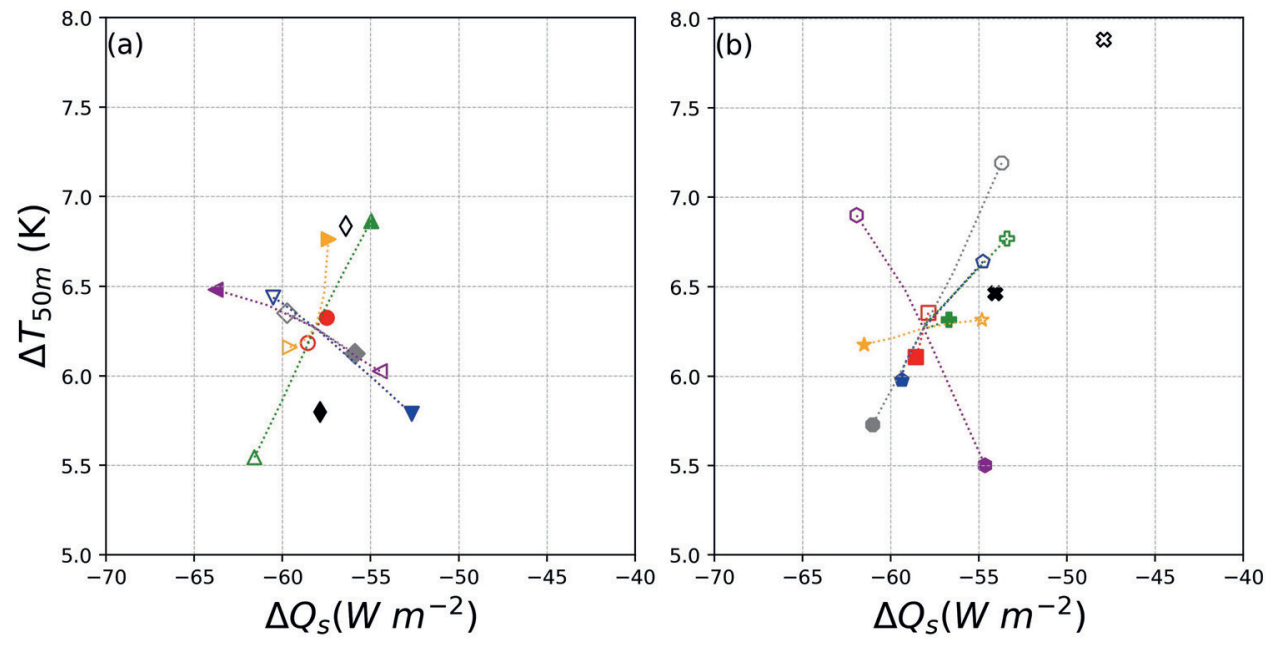

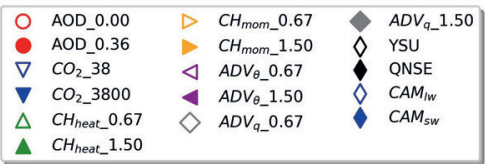

\begin{tabular}{|c|c|c|c|c|c|}
\hline$\square$ & $a_{\text {roof_ }} 0.10$ & 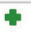 & $\lambda_{\text {wall_}} 1.05$ & 0 & $a_{\text {kanda_}} 0.50$ \\
\hline $\mathbf{\square}$ & $a_{\text {roof_ }} 0.30$ & मे & $f_{\text {urb_}} 0.75$ & 0 & $a_{\text {kanda_}} 1.40$ \\
\hline 0 & $C_{\text {wall_}} 0.6$ & $\star$ & $f_{\text {urb_}} 0.95$ & $\mathfrak{\infty}$ & BEP \\
\hline - & $C_{\text {wall__}} 2.4$ & 0 & $Q_{f_{-}} 10$ & * & $\mathrm{BEP}+\mathrm{BEM}$ \\
\hline क & $\lambda_{\text {wall_}} 0.15$ & 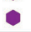 & $Q_{f_{-}} 70$ & & \\
\hline
\end{tabular}

Figure 3.3.5: As Figure 3.3.2, but for change in $\left(\Delta T_{50 m}\right)$ and the average storage heat flux $\left(\Delta Q_{s}\right)$ during night-time (19:00 UTC 23 July 2012 - 05:00 UTC 24 July 2012).

Increasing $A D V_{\theta}$ and $Q_{f}$ or decreasing $C H_{\text {heat }}$ and $a_{\text {kanda }}$ can decrease the night-time bias in the flux ratio. However, none can consistently correct biases in $Q_{H} / \Delta Q_{s}$ and $\Delta T_{50 m}$ at the same time.

\subsubsection{Intensity of turbulent mixing}

The intensity of turbulent mixing, indicated by the bulk Richardson $\left(R i_{b}\right)$ number (between the 1st and 2nd model levels) aids in understanding the coupling between the urban surface and the overlying boundary layer. This can help us understand how the changes in the flux partitioning affect this coupling and how is this translated to the changes we identify in $\Delta T_{50 m}$.

Most of the changes in atmospheric forcing (Figure 3.3.6a) and surface parameters (Figure 3.3.6b) limit the variability of the $R i_{b}$ between -0.71 to -0.58 during the day, with daytime changes in AOD, $C O_{2}$ and $A D V_{q}$ having small impact on $R i_{b}$ and minimal effect in $\Delta T_{50 m}$. However, $C H_{\text {heat }}$ causes large changes in $R i_{b}$ and $\Delta T_{50 m}$. Smaller increase $\Delta T_{50 m}$ occurs at lower $R i_{b}$ values and weaker turbulent intensity, while the opposite is true for higher $R i_{b}$ values. This is somewhat counter-intuitive, because an increase in $Q_{H}$ with larger values $C H_{\text {heat }}$ would have increase the turbulent intensity and $\Delta T_{50 m}$. However, the increase in 
$\mathrm{CH}_{\text {heat }}$ results in heat being transported more rapidly from the surface into the boundary layer, warming up the entire boundary layer and reducing the temperature gradient near the surface, driving $Q_{H}$ and $R i_{b}$ down during the day (Figure A.3.10). Changes in $A D V_{\theta}$ cause a small decrease in $R i_{b}$ for an increasing in $\Delta T_{50 m}$ (Figure 3.3.6a, A.3.6a,b). This is primarily caused by the increase in near surface temperature gradient as colder air is advected above the warm surface.

Surface parameter changes show a clear influence on the $\Delta T_{50 m}-R i_{b}$ parameter space, with a $0.2^{\circ} \mathrm{C}$ to $2.5^{\circ} \mathrm{C}$ change in $\Delta T_{50 m}$ for a 0.1 change in $R i_{b}$ (Figure 3.3.6b). The $a_{\text {kanda }}$, $a_{\text {roof }}$, and $Q_{f}$ have the largest impact on $R i_{b}$ during the day. These changes in $R i_{b}$ and $\Delta T_{50 m}$ primarily originate from a change in $Q_{H}$.

At night (Figure 3.3.6c) $A D V_{\theta}, C O_{2}$ and $C H_{\text {heat }}$ show the largest impact on $R i_{b}$ and $\Delta T_{50 m}$. Changes in $A D V_{\theta}$ cause an increased $\Delta T_{50 m}$ for weaker stability near the surface (smaller $R i_{b}$ ). The decrease in atmospheric stability is caused by the decrease in near surface temperature gradient, from the faster decrease in $T_{50 m}$ compared to changes in skin temperature, when strong negative $A D V_{\theta}$ is applied (Table 3.3.1b). This response when $A D V_{\theta}$ increases is from a stability feedback mechanism. This results in less radiative cooling and weaker atmospheric stability when daytime $\Delta T_{50 \mathrm{~m}}$ decreases. Under low wind conditions, the impact of the feedback mechanisms intensifies and results in lower nocturnal $\Delta T_{50 m}$ compared to the reference run (Figure A.3.6c). The remaining atmospheric forcing modification or surface parameter changes show an increase in nocturnal cooling rate, coinciding with also a faster heating rate during the day. Strong radiative cooling after sunset is the main driver for the stronger atmospheric stability in the experiment runs at night (Figures 3.3.6c,d).

Nocturnal surface parameter changes have a large impact on $R i_{b}$ and $\Delta T_{50 m}$, with changes in $Q_{f}$ and $\lambda_{\text {wall }}$ showing non-linear responses during the night. Changes in $C_{\text {wall }}, \lambda_{\text {wall }}$ and $a_{\text {kanda }}$ have a strong impact in $\Delta T_{50 m}$, which compensates for the larger daytime increase in $\Delta T_{50 m}$ (Figure 3.3.6b,d). This compensation leads to similar night-time $T_{50 m}$ for these surface parameters (Figure A.3.8a). Nocturnal $\Delta T_{50 m}$ is strongly dependent on the radiative cooling and the atmospheric stability near the surface, thus surface parameters which increase day-time $\Delta T_{50 m}$ result in stronger stability during the night. These findings support our hypothesis that a negative feedback mechanism exists between day time $\Delta T_{50 m}$ and nocturnal $\Delta T_{50 m}$.

\subsection{Physical ensemble analysis}

\subsubsection{Radiation schemes}

Using the CAM short-wave radiation scheme, instead of the reference RRTMG, increases the $S W_{\text {down }}$ bias to $51 \mathrm{Wm}^{-2}$, while reducing the $Q^{*}$ bias to $17 \mathrm{Wm}^{-2}$ (Figure 3.3.2a). 

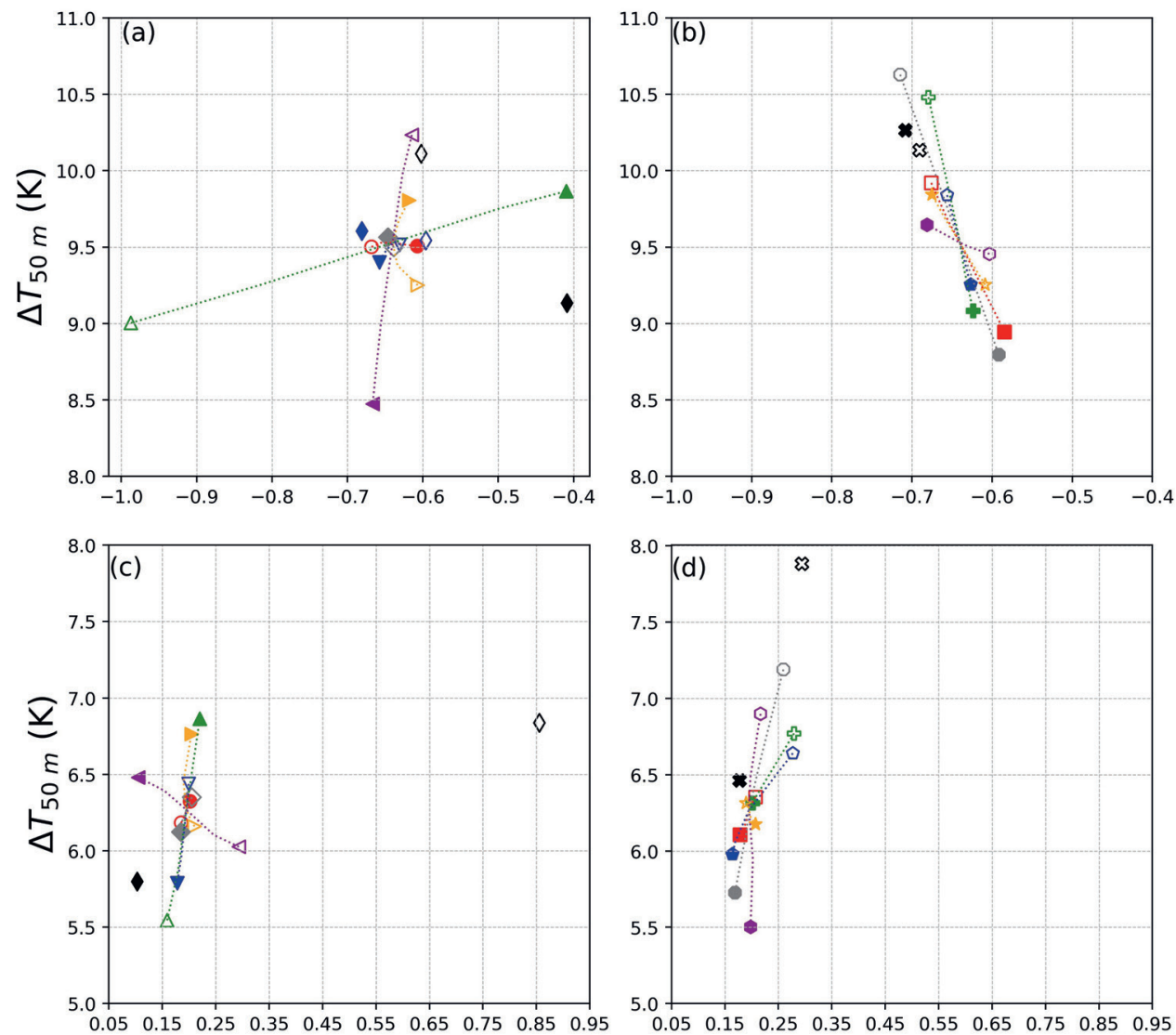

$R i_{b}(-)$

$R i_{b}(-)$

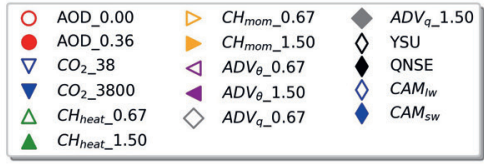

\begin{tabular}{|c|c|c|c|c|c|}
\hline$\square$ & $a_{\text {roor__ }} 0.10$ & $\Rightarrow$ & $\lambda_{\text {wall_}} 1.05$ & 0 & $a_{\text {kanda_ }} 0.50$ \\
\hline & $a_{\text {roor_}} 0.30$ & $\Delta$ & $f_{\text {urb_o }} 0.75$ & - & $a_{\text {kanda_1 }} 1.40$ \\
\hline 0 & $C_{\text {wall_}} 0.6$ & $\star$ & $f_{\text {urb__ }} 0.95$ & 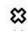 & BEP \\
\hline - & $C_{\text {wall_}} 2.4$ & 0 & $Q_{f_{-}} 10$ & * & $B E P+B E M$ \\
\hline \& & $\lambda_{\text {wall_}} 0.15$ & 0 & $Q_{f_{-}} 70$ & & \\
\hline
\end{tabular}

Figure 3.3.6: As Figure 3.3.3, but for changes in $50 \mathrm{~m}$ air temperature $\left(\Delta T_{50 \mathrm{~m}}\right)$ and the average bulk Richardson number $R i_{b}$.

The difference between the schemes aligns with the changes in AOD, which indicates that $C A M_{s w}$ in WRF does not fully account for scattering by aerosols. This physical difference will mask the biases in net long-wave radiation when the $C A M_{s w}$ is used, making it more difficult to identify remaining biases, unless each radiation flux component is treated individually. Comparing $C A M_{l w}$ and $R R T M G_{l w}$ an increase in daytime $L W_{\text {down }}$ bias of $8 \mathrm{Wm}^{-2}$ is identified, causing an increase in $Q^{*}$ bias. The $C A M_{l w}$ aligns with the impact of a decrease in $\mathrm{CO}_{2}$ concentration. That does not indicate an absence of $\mathrm{CO}_{2}$ 
effects on $L W_{\text {down }}$ in $C A M_{l w}$, but is related to a different physical description of longwave radiation between the schemes and it could originate from not accounting fully for water vapor effects on $L W_{\text {down }}$ and from the few spectral bands used compared to the RRTMG scheme. The exact same biases from $C A M_{s w}$ and $C A M_{l w}$ are seen throughout Figures 3.3.3a,c; 3.3.4a,c; 3.3.5 and 3.3.6) indicating the importance of the radiation scheme choice.

\subsubsection{Boundary layer schemes}

Changes in atmospheric forcing do not clearly explain daytime deviations between the different boundary layer parameterization schemes used (YSU,QNSE and the reference MYJ). Although initially $A D V_{\theta}$ appears to be the primary difference between YSU and the other two schemes, this is misleading as advection is the same for all. Changes in $\mathrm{CH}_{\text {heat }}$ and $\mathrm{CH}_{\text {mom }}$ cannot explain the variations, neither can changes in $\mathrm{CO}_{2}$ or AOD. Considering that runs with different boundary layer schemes show small variation in $Q^{*}$ (Figure 3.3.3a), but large variation in $\Delta T_{50 m}$, the differences should be primarily driven by the physical description of turbulent processes. Indeed for the daytime the main difference between YSU and MYJ seems to be the explicit inclusion of entrainment of heat in the YSU scheme and a slightly enhanced surface $Q_{H}$, potentially due to the different surface layer scheme YSU is coupled to. Moreover, decreasing $A D V_{\theta}$ does produce the same effect as the explicit inclusion of entrainment, because both increase mean boundary layer temperature (consistent with the larger $\Delta T_{50 m}$ for YSU) and boundary layer height in YSU (not shown). Thus, additional attention should be given not to misinterpret compensating effects from these two physical processes. At night the YSU scheme impact is similar to effects of increased $C H_{\text {heat }}$ (Figure 3.3.3c). This is expected as increased $\mathrm{CH}_{\text {heat }}$ indicates stronger nocturnal stability via enhanced radiative cooling due to a stability related feedback mechanism (section 3.3.4 and A.3.2,3).

Differences in MYJ and QNSE are minimal for $Q^{*}$ (Figures 3.3.3a,c) and the $Q_{H} / \Delta Q_{s}$ partitioning (Figures 3.3.4a,c) at both time periods. However, substantial differences occur between them in $\Delta T_{50 m}$ and stability (Figure 3.3.6a,c). Runs with the QNSE schemes have a smaller diurnal cycle of temperature and instability, reduced buoyancy flux during the day and weaker stability at night. The nocturnal deviation from MYJ is caused by stronger wind shear in the QNSE schemes, which appears to be related with lower $\mathrm{CH}_{\text {heat }}$ and $\mathrm{CH}_{\text {mom }}$ at night-time to reduce the radiative cooling at the surface.

\subsubsection{Urban canopy models}

The different model results from SLUCM, BEP+BEM and BEP model can be explained reasonably well with the surface parameters differences. SLUCM can match the daytime $\mathrm{BEP}+\mathrm{BEM}$ results reasonably well by decreasing $a_{\text {roof }}$ (to 0.12) and $a_{\text {kanda }}$ (to 0.80) and increasing $C_{\text {wall }}$ (to $1.8 * 10^{6} \mathrm{~J} \mathrm{~m}^{-3} \mathrm{~K}^{-1}$ ). Decreasing the SLUCM $a_{\text {roof }}$ is consistent with 
the lower bulk albedo for the BEP+BEM and BEP models, which have different physical description of the urban morphology and shading. The $a_{\text {kanda }}$ change increases the modeled $2 \mathrm{~m}$ exchange coefficient of heat for SLUCM, which is lower than in BEP+BEM. The increase in SLUCM $C_{\text {wall }}$ increases the $\Delta Q_{s}$, and can compensate the lack of a building energy model and air-conditioning cooling (present in BEP+BEM), which increases the heat capacity of the urban fabric as internal building temperatures remain lower. Exclusion of the BEM module in the multi-layer scheme leads to substantially larger $Q_{H}$, and lower thermal storage. To match BEP with SLUCM in addition to the changes in $a_{\text {kanda }}$ and $a_{\text {roof }}$ are needed as well as, a decrease in $\lambda_{\text {wall }}$ (to 0.45 ) and a reduction of $Q_{f}$ to 0 $W m^{-2}$ (BEP does not account for $Q_{f}$ ).

These modifications reduce the differences between SLUCM, BEP+BEM and BEP, causing a daytime $\Delta T_{50}$ difference reduction to $0.20^{\circ} \mathrm{C}$ (from $0.75^{\circ} \mathrm{C}$ ) and a $0.06^{\circ} \mathrm{C}$ reduction at night (from $0.25^{\circ} \mathrm{C}$ ). $Q^{*}$ is only improved during the day and becomes identical to $\mathrm{BEP}+\mathrm{BEM}$ with large reduction in the differences between the $S W_{u p}$ and $L W_{u p}$ between the two schemes. The same is true for $Q_{H} / \Delta Q_{s}$ and the $R i_{b}$, which indicates more similarity in the surface fluxes and the near surface atmospheric stability. However, at night substantial differences remains in $Q^{*}$, due to higher skin temperatures in SLUCM, and also in $Q_{H} / \Delta Q_{s}$ as $Q_{H}$ in SLUCM lower compared to BEP+BEM.

\subsection{Discussion}

This analysis identifies the model response to changes in atmospheric forcing and urban surface parameter for a specific model configuration (section 3.2.2) and for a specific UCM (SLUCM). The same sensitivity analysis with different model configurations (e.g. different NWP, or reference parameterization schemes) and UCMs are anticipated to lead to different model responses. This is to be expected due to differences in model setups and UCMs. Hence, we recommend other UCMs with more complex (i.e multi-layer schemes) and more simplified (e.g. bulk schemes) physical description of the urban surface to be tested. Similarly different case studies, urban areas and predominant meteorological conditions (e.g. cloudy/rain period, different geostrophic wind speeds) need to be considered.

To ensure our analysis is not day-specific, we compare the model responses between the first and the second day of the SUBLIME case study. During the second day geostrophic wind speed is substantial lower, thus sensitivity of the model's response to geostrophic wind is also tested. The model response remains similar between the two days, with the few differences linked to the non-linear feedback between daytime $T_{50}$ and nocturnal atmospheric stability, causing non-linear behaviour for some variables due to the generally larger $R i_{b}$ during the second day, an effect of the lower wind speeds.

The plausible uncertainty range in atmospheric forcing and surface parameters (as presented in section 3.2.3) is based on previous reported sensitivity tests and uncertainty 
estimates (Loridan et al., 2010; Wang et al., 2011; Zhao et al., 2014). It could however under- or over-sample the uncertainty range for specific parameters leading to skewed model sensitivity. It is expected that such an effect would be more profound on the actual range of the sensitivity and not so much on the orientation or linearity of the model's response in the parameter space. However, under low wind conditions the response for some parameters might be non-linear due to stability effects. Therefore, careful selection for the uncertainty range and the frequency of sampling from the parameter range is essential. For $\mathrm{CH}_{\text {heat }}$ and $\mathrm{CH}_{\text {mom }}$, changes in other atmospheric forcing or surface parameters will affect the calculated values, but their effect is small compared to changes in the multiplication factors.

The 1D-WRF-Noah setup and the boundary layer scheme influence the outcome of the sensitivity analysis. In reality a boundary layer will react differently to changes in physical properties of the underlying surface. For example, any variation in the urban surface temperature over the urban area will likely result in a change of temperature advection and also wind speed due to changes in pressure gradient. Such processes can not be represented in a single column model where advection is prescribed, but will require 3D simulations. Such limitations in the representation of the overlying atmosphere might exclude feedback mechanisms that could change the sensitivity reported in this study.

Following Tsiringakis et al. (2019), we used the $L W_{u p}$ calculated in the SLUCM rather than the WRF reference as the $L W_{u p}$ from the long-wave radiation scheme uses an average aerodynamic surface temperature instead of the radiative skin temperature of the facets. SLUCM calculates this aerodynamic surface temperature diagnostically from the air temperature, $Q_{H}$ and the modeled exchange coefficient of heat. During our analysis we found that this aerodynamic surface temperature varies substantial from the radiative skin temperature of the urban facets. Since the BEP+BEM and BEP schemes uses a similar approach to calculate the $L W_{u p}$ (i.e. via the radiative skin temperature) we used the same approach for WRF-Noah-SLUCM. Note this difference does not affect $Q^{*}$ at the surface, as this $L W_{u p}$ is from the urban scheme, using the radiative temperature.

This study does not optimize the model performance, but tries to understand how uncertainty in forcing and parameters affect key physical processes in the urban surface and overlying atmosphere. However, if one is mainly interested in improving model performance through optimization there are a series of potential techniques to do so. Loridan et al. (2010) and Zhao et al. (2014) used ensemble Kalman filtering and Monte-Carlo approach to optimize the urban surface parameters in order to improve the SLUCM' s performance, with very promising results. For off-line or even limited single-column model simulation that might be computationally possible, but for full 3D model simulations the computational cost might be prohibitive. Inverse modeling might be more efficient, but requires an adjoint model of WRF, which to our knowledge is not currently available.

Here we use modeled $\Delta T_{50}$ rather than the modeled $T_{50}$ (following Sterk et al. (2013)) 
to minimize the impact of biases introduced during spin-up phase and to allow analysis of model response to changes in atmospheric forcing and surface parameters separately for day and night. Thus we are able to easily distinguish compensating effects on $T_{50}$ between day and night (e.g. changes in $\lambda_{\text {wall }}$ and $C H_{\text {heat }}$ ) or carryover effects from day to night (e.g. $A D V_{\theta}$ and $Q_{f}$ ). Moreover, the changes in $\Delta T_{50}$ during day or night are directly linked with the changes in radiation and surface energy balance. This allows us to identify more accurately, which atmospheric forcing or surface parameter changes explains the bias between model and observations or differences between different model setups.

\subsection{Conclusions}

In a coupled NWP-UCM model setup, surface parameters and atmospheric forcing are the primary sources of uncertainty and strongly affect model performance. With WRF-NoahSLUCM we investigate the impact of these during a 1-day clear-skies period in London. The impact of change in atmospheric forcing and surface parameters to the surface radiative balance, energy partitioning and intensity of turbulent mixing are calculated together with the coupling between the surface and the overlying atmosphere.

Both atmospheric forcing and surface parameters changes impact the model's performance. For the radiative balance, AOD, $a_{\text {roof }}, \mathrm{CO}_{2}$ and $a_{\text {kanda }}$ are the most influential parameters, each impacting different terms. $S W_{u p}$ and $L W_{u p}$ cause the bias in modeled $Q^{*}$. Correcting for the radiative bias improves the radiative heating and radiative cooling performance. For the surface energy flux partitioning, the model has the largest response to changes in $C H_{\text {heat }}, a_{\text {kanda }}, Q_{f}, C_{\text {wall }}$ and $\lambda_{\text {wall }}$. Changes in near surface atmospheric stability is comparable in magnitude from most changes in atmospheric forcing and surface parameters, with different orientation for each of the sources. Changes in $C H_{\text {heat }}$ have the largest impact on daytime $R i_{b}$, while $A D V_{\text {heat }} a_{\text {kanda }}, C_{\text {wall }}$ and $\lambda_{\text {wall }}$ are more critical at night. A feedback mechanism between increasing daytime $T_{50 m}$ and increase in nocturnal radiative cooling is identified. Its intensity is strongly depended on the wind shear.

We identify some compensating effects between atmospheric forcing and surface parameters changes in this analysis. This is mainly for surface parameters related to theral storage (e.g $f_{\text {urban }}, C_{\text {wall }}$ and $\lambda_{\text {wall }}$ ) and between $C H_{\text {heat }}$ and $a_{\text {kanda }}$, which are linked to the surface-atmosphere coupling. Both AOD and $a_{\text {roof }}$ impacts on $Q^{*}$ and surface flux partitioning are similar. The reported compensating, evident from single-variable analysis, are reduced by using the 2 -variable space analysis (the so-called the processes diagrams). Various atmospheric forcing and surface parameter changes have similar effects, if not separated by time of day. This supports (Best \& Grimmond, 2015) suggestion to analyze model responses under different turbulence regimes. 
We highlight that it is possible to identify physical description differences between the schemes used in our WRF-Noah-SLUCM setup. This is easier when key physical mechanisms are missing from a scheme (e.g. lack of aerosol effects on $S W_{\text {down }}$ ), but is more difficult when the process is not covered by the atmospheric forcing range of analysis (e.g. explicit entertainment flux in YSU compared to MYJ). This analysis can also identify compensating effect between atmospheric processes, as demonstrated by the fact that decreasing $A D V_{\theta}$ shows a similar effect to including explicit entertainment. We could identify differences in the UCM schemes through the use of changes in the surface parameters and link them to differences in the physical complexity of the schemes, which allows to link uncertainty to either changes in atmospheric forcing or surface parameters. However, this approach has clear limitation (e.g. inability to explained the difference in nocturnal $Q^{*}$ between SLUCM and BEP+BEM).

\section{Acknowledgements}

This research has been funded by the NWO grant no. 864.14.007. The authors would like to thank the ECMWF for providing operational analysis data, the University of Wyoming for the radio-sounding data and also NOAA for providing data for the WMO stations, all of which were used to create the forcing for the on-line model. Moreover, we would like to NASA/EOSDIS for providing AOD data via the EOSDIS Worldview site and the various grants that support the London observations (LUMA). Finally, we would like to thank all the co-authors of the SUBLIME case study, which is utilized by this paper. The dataset for this research (Tsiringakis et al., 2020) is publicly available in the Zenodo repository (https://zenodo.org/record/3897222, doi: 10.5281/zenodo.3897222). 



\section{Chapter 4}

\section{Interactions between the nocturnal low-level jets and the urban boundary layer: a case study over London}

This chapter is based on:

Tsiringakis, A., Theeuwes, N., Barlow, J. and Steeneveld, G. J. (2021). Interactions between the nocturnal low-level jets and the urban boundary layer: a case study over London. under review. 


\section{Abstract}

Understanding the physical processes that affect the turbulent mixing in the nocturnal urban boundary layer (UBL) is essential to improve forecast of air quality and heat in urban areas. The low-level jet (LLJ) is one such process that can affect turbulence in the nocturnal UBL. In this study we investigate the interaction of a regional LLJ with the UBL during a 60-hour case study period. We use observations from two Doppler Lidars and results from two high-resolution numerical weather prediction models (Weather Research \& Forecasting model and UKV Met Office Unified Model) to study differences in the occurrence frequency, height, wind speed and fall-off of the LLJs between an urban (London, UK) and a rural (Chilbolton, UK) site. We find that LLJs are elevated $(\approx$ $70 \mathrm{~m}$ ) over London, due to the deeper UBL, while the wind speed and fall-off are slightly reduced with respect to the rural LLJ. Utilising two idealized experiments in WRF we isolate urban from non-urban (i.e topographic, proximity to coast) effects on the LLJs over London. We find that topography strongly affects the LLJ characteristics (height, falloff, and speed), but there is still a substantial urban influence. Finally, we find that the presence of the LLJ over the nocturnal UBL enhances the shear-produced TKE, maintains part of the daytime vertical mixing and slows down the collapse of the daytime UBL. 


\subsection{Introduction}

Urban boundary layers (UBL) differ substantially in their depth and vertical structure from their rural counterparts (Pal et al., 2012; Barlow, 2014), due to differences in the surface energy balance and the turbulent flow over the urban surface (Arnfield, 2003; Barlow et al., 2015). One key difference is the increased vertical turbulent mixing, which can lead to a delayed collapse of the day-time UBL and results in a deeper nocturnal UBL (Pal et al., 2012). The increased nocturnal turbulent mixing is a result of turbulent kinetic energy (TKE) produced by : a) buoyancy, generated via the slow release of heat stored in buildings during daytime and anthropogenic activities, and b) shear, generated via interactions of the wind and urban surface. Understanding processes and phenomena that affect the nocturnal TKE production over cities is essential to better understand and represent the development of the nocturnal UBL. One such phenomenon that has the potential to influence the intensity of turbulent mixing in the nocturnal UBL is the low-level jet (LLJ) (Lundquist \& Mirocha, 2008; Barlow et al., 2015).

Low-level jets are super-geostrophic wind maxima that occur near the surface, usually at the top of the nocturnal boundary layer (Blackadar, 1957; Baas et al., 2009). They are formed through two distinct mechanisms: a) inertial oscillations (IO) due to the collapse of turbulent mixing after sunset (Blackadar, 1957) and b) baroclinicity from local topographic differences or large-scale synoptic forcing (Holton, 1967; Kotroni \& Lagouvardos, 1993). Shapiro et al. (2016) unified both mechanisms and showed that their interaction leads to strong LLJs over the Great Plains in the US. LLJs are known to increase shear-produced TKE (Banta et al., 2003, 2006; Wang et al., 2007; Lundquist \& Mirocha, 2008) and potentially lead to enhanced entrainment in the nocturnal UBL. In order to study LLJs, previous studies employed in-situ observations (i.e. Doppler Lidar, SODAR) (Banta et al., 2003; Wang et al., 2007; Baas et al., 2009; Kallistratova \& Kouznetsov, 2012; Barlow et al., 2015; Banakh \& Smalikho, 2018) and/or models (i.e. NWP models, LES) (Storm et al., 2009; Park et al., 2014; Vanderwende et al., 2015).

Many studies have identified differences between urban and rural LLJs. Kallistratova \& Kouznetsov (2012) showed that LLJs occur less often and at a higher height over Moscow compared to a nearby rural site. Similar conclusions were drawn by Wang et al. (2007) during the Joint Urban (JU03) campaign. LLJs were shown to increase of the depth of the nocturnal UBL and the intensity of urban heat island (UHI) (Wang et al., 2007; Lundquist \& Mirocha, 2008; Hu et al., 2013a). Barlow et al. (2015) suggested that LLJs can increase vertical mixing over urban areas, potentially leading to a formation of an upside-down boundary layer, where mixing is driven from the shear-generated TKE at the top of the nocturnal inversion. This hypothesis was also supported by several other studies that showed an increase in shear-produced TKE, below the LLJ (Banta et al., 2003, 2006; Lundquist \& Mirocha, 2008; Banakh \& Smalikho, 2018). Hu et al. (2013a) showed that this downward mixing was caused by the nocturnal low-level jet enhances the 
near-surface ozone concentrations. Correctly accounting for the impact of LLJs on the vertical mixing and UBL depth can have strong implications for forecasting air quality over urban areas.

Despite this progress, our understanding of the interaction between the LLJs and the complex urban morphology is still limited (Lundquist \& Mirocha, 2008; Barlow et al., 2015). Barlow et al. (2015) hypothesised that LLJ could be advected over London from nearby rural areas, rather than formed directly above the city. Yet, it is still unclear how the enhanced TKE and the weaker stability over urban areas affect the lifetime, occurrence frequency and characteristics (fall-off, height, speed) of the LLJs. The effects of LLJ-induced shear on the vertical mixing and structure of the nocturnal UBL are still under investigation. The local topographic heterogeneity in or near urban areas (hills, coast lines etc.) can also affect the LLJs. This inhibits the investigation of LLJs solely through observations as the spatial distribution of LLJ characteristics cannot be fully captured. This requires a combined approach that compliments field measurements with numerical modelling, which will characterise the spatial distribution of the LLJ and allow for sensitivity experiments (e.g. urban energy balance, local topography).

In this study we use a combined approach (two Doppler Lidars and two NWP models) to investigate differences in the LLJ characteristics between an urban (London) and a rural (Chilbolton) site. Section 4.2 describes the methodology. Then, an evaluation of both NWPs against in-situ Doppler Lidar observations is conducted (Sect. 4.3), followed by the main analysis of LLJ differences between the two sites (Sect. 4.4). A spatial analysis of LLJ characteristics over London (Sect. 4.5) is followed by idealized experiments used to quantify non-urban (topographic, coastline etc.) effects on LLJ characteristics over London (Sect. 6), before the quantification of LLJs effects on vertical mixing in the UBL (Sect. 4.7), followed by a discussion (Sect. 4.8) and conclusions (Sect. 4.9).

\subsection{Methodology}

\subsubsection{Case Study Selection}

A 60-hour period (0000 UTC 14 May 2019 - 1200 UTC 16 May 2019) is selected as case study. The period is characterized by a high pressure system (Fig. A.4.1, A.4.2), located over the North sea, causing easterly synoptic flow over south-east England and little cloud cover. During the course of the 60-hour period the high pressure system moves to the north-east resulting in a decrease in baroclinicity during the second night (15-16 May 2019, Fig. A.4.1). This period was selected as it is one of the few periods with clearly defined LLJs detected over both Doppler Lidar sites (London and Chilbolton) without frontal passages that could influence the LLJ formation and characteristics. 


\subsubsection{Model description: Weather Research and Forecasting model (WRF)}

Here we use the 3D WRF-ARW v4.1 model (Skamarock et al., 2019) in a two domain configuration (Fig. 4.2.1b), with an outer domain (7.5 km horizontal resolution, 200x200 grid-cells) and a one-way nested inner domain (1.5 km resolution, 201x201 grid-cells) centred over London. This configuration ensures that the high pressure system over the North-Sea is resolved in the outer domain, while maintaining high resolution necessary to resolve spatial heterogeneity that can influence LLJs in the inner domain. The model runs for the 60-hour period, with the first 12 hours considered as spin-up time. We use 90 vertical hybrid-eta grid levels up to $100 \mathrm{hPa}$, with increased vertical resolution near the surface (10 levels in the lowest $500 \mathrm{~m}$ ). Initial conditions for both domains and boundary conditions (outer only) are provided using the 6-hourly ECMWF operational analysis (10 $\mathrm{km}$ resolution) data.
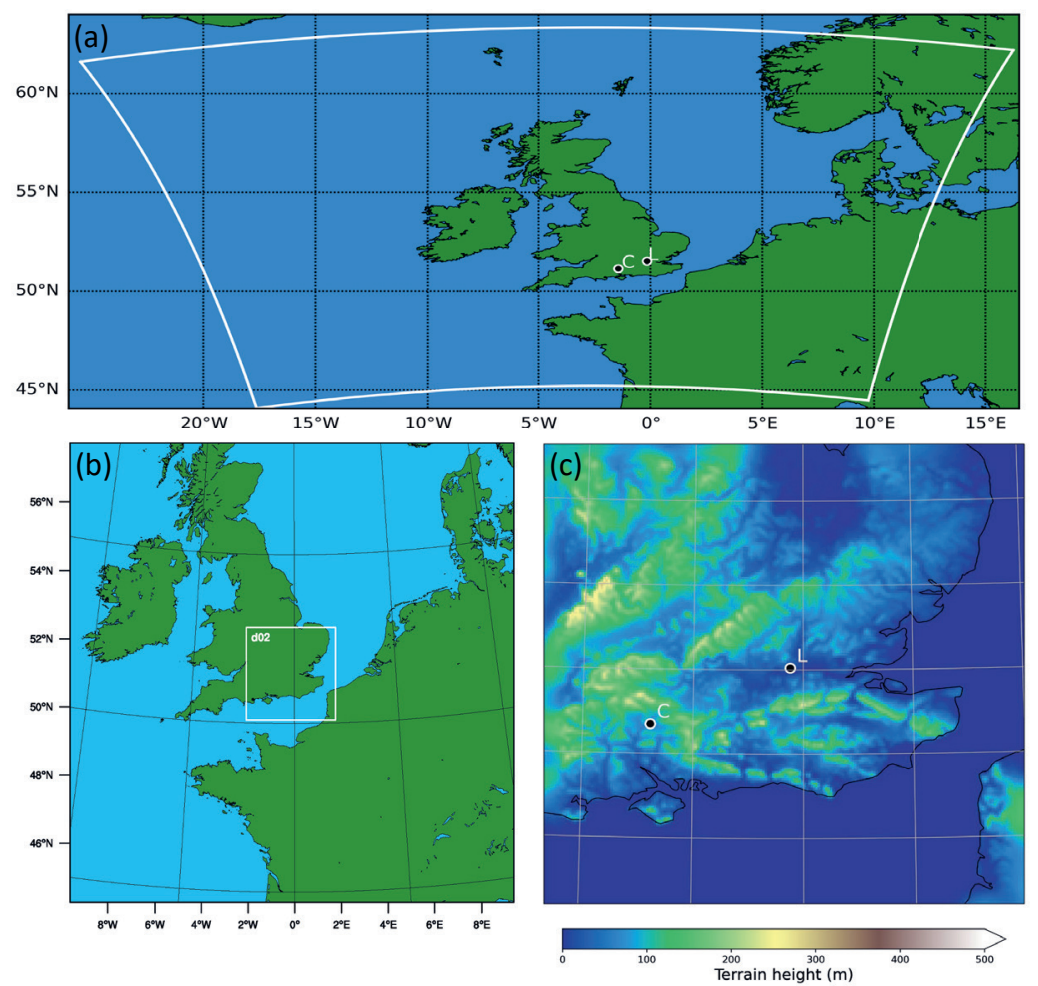

Figure 4.2.1: Illustrations of (a) the model domain of the UKV model, (b) the outer (d01) and inner (d02) domains of the WRF model and (c) the terrain height (m) of the inner WRF model domain. The locations of the London and Chilbolton Doppler Lidar sites are designated with the dots and the corresponding starting letter of each site. 
In the outer domain, the surface and boundary layer are parametrized using the revised MM5 (Jimenez et al., 2012) and Mellor-Yamada Nakanishi Niino (MYNN) Level 2.5 (Mikio \& Hiroshi, 2009) schemes, which is shown to perform well in recent LLJ studies (Kalverla et al., 2019a,b). Moreover, MYNN allows for TKE advection, which is crucial to capture the transport of TKE downwind of the urban area and it allows for a smoother transition in UBL height between urban and rural grid-cells. Convection is parameterized using the Tiedtke scheme (Zhang et al., 2011), while for microphysics the Thompson et al. (2008) scheme is used. Shortwave and longwave radiation are parameterized using the RRTMG schemes (Iacono et al., 2008). The urban surfaces are represented using the single-layer urban canopy model (Kusaka et al., 2001; Chen et al., 2011a) with the Noah-LSM scheme (Chen \& Dudhia, 2001) representing the vegetated land-surface processes.

The inner domain uses the same physics suite, with a few exceptions. For the boundarylayer scheme (MYNN) the eddy-diffusivity-mass-flux functionality and the advection of TKE are activated (Olson et al., 2019). Kalverla et al. (2019a) found that the MYNNEDMF scheme is able to reproduce well LLJs over the North-Sea. Convection is explicitly resolved in the inner domain. The default WRF MODIS land-use (LU) class is modified by adding two additional urban LU classes to better represent the urban morphology of London in the inner domain. The 3 dominant urban LU classes (low, middle and high density urban areas) correspond to local climate zones (LCZs) 6, 5 and 2, with a spatial distribution and surface parameters following the WUDAPT LCZ classification of London (https://wudapt.cs.purdue.edu/wudaptTools/default/getlcz). The topography in the inner WRF domain is depicted in Fig. 4.2.1c. Note that there are hills $(100-200 \mathrm{~m}$ a.g.l) north and south of London, while over the central part of the city the elevation is rather low.

\subsubsection{Model description: high-resolution UK model (UKV)}

The Met Office operational limited-area forecasts for the UK (UKV) are run using a limited area set-up with 70 vertical levels and a grid spacing of $1.5 \mathrm{~km}$ at the centre of the domain, which becomes more coarse towards the edges (Fig. 4.2.1a). The forecast is run every three hours with a 3D-VAR data assimilation cycle. This study uses the $09 \mathrm{z}$ forecasts. The atmospheric turbulence is parametrized using a blending approach described by Boutle et al. (2014). The scheme blends between a 1D non-local boundarylayer scheme by Lock et al. (2000) and a Smagorinsky-Lilly scheme dependent on the turbulent length scale and the grid length. The land surface exchange is parameterized using JULES (Joint UK Land Environment Simulator; Best et al., 2011), that uses a tiling approach with 10 tiles. The radiation and fluxes from the urban tile are calculated using MORUSES, which is a single layer urban canyon scheme (Met Office-Reading Urban Surface Exchange Scheme; Harman et al., 2004; Porson et al., 2010). Finally, there is no shallow convection scheme and the cloud scheme by Smith (1990) is used. 


\subsubsection{Doppler Lidar observations}

Wind profiles are observed at two locations, one in the centre of London and one at a rural site $100 \mathrm{~km}$ southwest of the London site (Table 4.2.1). In London, a 1.5 $\mu \mathrm{m}$ HALO Photonics Streamline Doppler Lidar is located at the London Southbank University (LSU) roof-top site $\left(51^{\circ} 29^{\prime} 53.4^{\prime \prime} \mathrm{N}\right.$; $\left.0^{\circ} 06^{\prime} 07.2^{\prime \prime} \mathrm{W}\right)$. The roof level is $36.5 \mathrm{~m}$ above sea level and $33.5 \mathrm{~m}$ above ground level. The mean building height in a $1 \mathrm{~km}$ radius of the site is 12.8 $\mathrm{m}$, with a standard deviation of $9.5 \mathrm{~m}$. The tallest building in the immediate vicinity is approximately $140 \mathrm{~m}$ above ground level $200 \mathrm{~m}$ southeast of the Doppler Lidar. The London wind profiles are calculated using a 6-point Velocity Azimuth Display (VAD) scan at $75^{\circ}$ elevation, which occurs every 6 minutes. Additional filtering to remove points with wind speed error larger than $2 \mathrm{~m} \mathrm{~s}^{-1}$ and individual error in either the $\mathrm{u}$ or $\mathrm{v}$ wind component larger than $1.5 \mathrm{~m} \mathrm{~s}^{-1}$ is applied. Then the data are averaged to halfhourly values using the 5 6-minute scans which occur around the half-hourly points (i.e. plus/minus 15 minutes around the 00:00 timestamp).

In Chilbolton $1.5 \mu \mathrm{m}$ HALO Photonics Streamline Pro Doppler Lidar is surrounded by grasslands $\left(51^{\circ} 08^{\prime} 40.2^{\prime \prime} \mathrm{N} 1^{\circ} 26^{\prime} 13.2^{\prime \prime} \mathrm{W}, 90 \mathrm{~m}\right.$ above sea level). At this site, a 24-point VAD scan at $75^{\circ}$ elevation occurs every 10 minutes from which wind profiles are derived. For both Doppler Lidars, backscatter corrections and error estimates are calculated following Manninen et al. (2016) and Vakkari et al. (2019). A similar filtering and averaging algorithm is applied in the Chilbolton data, but using 3 10-minute wind profiles to compute the half-hourly values at each time step.

Mixing heights are also derived from the Doppler Lidar scans. In between other scan patterns, the Doppler Lidars are operating in vertical stare mode, at an elevation angle of $90^{\circ}$. This scan returns the vertical velocity at a high temporal resolution, i.e. $0.5-0.625$ $\mathrm{Hz}$ for the London site and $0.048-0.055 \mathrm{~Hz}$ at Chilbolton. From the high resolution vertical velocity data, higher order statistics are calculated over time periods of 30 minutes, i.e. variance, skewness, kurtosis. The vertical velocity variance is used as a measure for the turbulent mixing in the boundary layer and a simple threshold is used to determine the top of the mixed layer following previous studies (Barlow et al., 2015; Halios \& Barlow, 2018; Theeuwes et al., 2019). Data where the signal to noise ratio was low $(S N R+1<1.01)$ are filtered out. In order to compute the vertical velocity statistics for each 30-minute time period over each range gate, more than $30 \%$ of the data needs to be available. To calculate the sensitivity of the mixing height to the threshold $0.1 \mathrm{~m}^{2} \mathrm{~s}^{-2}$, the value was perturbed by $30 \%$. For each averaging period 21 threshold values ranging from 0.069 to $0.129 \mathrm{~m}^{2} \mathrm{~s}^{-2}$ were used and the height where the velocity variance drops below each threshold is stored as a possible mixing height. The final mixing height is the median of the 21 possible mixing heights. At the London site a reduction of signal intensity at the capping inversion limited the vertical velocity variance calculations during the deep convective boundary layer around noon. For those times the mixing height was determined 
Table 4.2.1: Properties of the two Doppler Lidars used in this study

\begin{tabular}{r|cc} 
& London & Chilbolton \\
\hline Type & 3, HALO Streamline & 118, HALO Streamline Pro \\
Wavelength & $1.5 \mu \mathrm{m}$ & $1.5 \mu \mathrm{m}$ \\
Pulse repetition rate & $20 \mathrm{kHz}$ & $10 \mathrm{kHz}$ \\
Range resolution & $18 \mathrm{~m}$ & $30 \mathrm{~m}$ \\
Maximum range & $3600 \mathrm{~m}$ & $9600 \mathrm{~m}$ \\
Focus Stare & $\infty$ & $\infty$ \\
Focus VAD 75 & $1000 \mathrm{~m}$ & $\infty$ \\
Lens diameter & $6 \mathrm{~cm}$ & $5 \mathrm{~cm}$ \\
\hline
\end{tabular}

from the signal intensity. The average signal intensity over a 30 -min period was vertically smoothed with a 7 point moving average. The minimum gradient of the smoothed signal was determined to be the mixing height, where mixing heights from the vertical velocity variance profiles were missing.

\subsubsection{LLJ detection and characterization}

LLJs are identified using a modified version of the Baas et al. (2009) algorithm. A LLJ is defined when a low-level wind maximum $\left(>4 \mathrm{~m} \mathrm{~s}^{-1}\right.$ ) occurs (below $650 \mathrm{~m}$ ) that has at least $1.5 \mathrm{~m} \mathrm{~s}^{-1}$ (or 15\%) higher wind speed than the wind in the overlying vertical levels (up to a height of $900 \mathrm{~m})$. Other studies use slightly modified fall-off criteria $\left(2.0 \mathrm{~m} \mathrm{~s}^{-1}\right.$ in Baas et al. (2009); Kalverla et al. (2017). However, Kalverla et al. (2019b) showed that the $2 \mathrm{~m} \mathrm{~s}^{-1}$ fall-off threshold might erroneously exclude LLJs occurring at higher heights $(>400 \mathrm{~m})$. Considering that LLJs over urban areas often occur above 400m (Lundquist \& Mirocha, 2008; Barlow et al., 2015; Klein et al., 2016) it is essential to use the $1.5 \mathrm{~m} \mathrm{~s}^{-1}$ $\mathrm{m}$ criterion to ensure that we correctly capture urban LLJs and have sufficient samples of LLJs across different heights to be able to compare differences in LLJ characteristics between urban and rural areas.

The LLJ characteristics identified using the LLJ detection algorithm are: a) the occurrence frequency, b) the fall-off, c) the peak jet speed and d) the LLJ height. The algorithm is applied in each grid-cell and at every model output time step (30 minutes in WRF, 1h in UKV). This allows both the spatial and temporal distribution of LLJs characteristics to be tracked. Model and Doppler Lidar data are interpolated to the same vertical levels using a simple linear interpolation before the LLJ algorithm is applied. The vertical levels start at $25 \mathrm{~m}$ a.g.l with a vertical resolution of $25 \mathrm{~m}$ until $600 \mathrm{~m}$ and a $50-\mathrm{m}$ resolution until $900 \mathrm{~m}$. Both models have a high vertical resolution near the surface and thus differences between linear and more complex polynomial (i.e. cubic) interpolation are small $\left(<0.1 \mathrm{~ms}^{-1}\right)$. 


\subsection{Model Evaluation}

\subsubsection{LLJ characteristics for London (LSU) and Chilbolton sites}

LLJs over London and Chilbolton are identified (Fig. 4.3.2c,f) during both nights of the case study. Unfortunately, the low aerosol concentrations, due to regionally clean air during the case study, gave insufficient SNR during the first night for both sites. Thus, no observations of wind speed above $450 \mathrm{~m}$ are available during the initial LLJ formation the first night of the case study. Nevertheless, it is still possible to identify LLJs during the first night in the Doppler Lidar observations at both sites starting at 0000 UTC 15 May 2019 over the urban site and 2200 UTC 14 May 2019 over the rural site. The LLJ lasts until 0700 UTC 15 May 2019 both sites. During the second night the LLJ is identified slightly earlier (2300 UTC 15 May 2019) over the urban site compared to the rural one (0000 UTC 15 May 2019), while both LLJs last for 7 hours.

During the first night the initial formation height for the LLJ was higher over London (450 m vs $350 \mathrm{~m})$, while the jet speed was higher over Chilbolton $\left(14.3 \mathrm{~m} \mathrm{~s}^{-1}\right.$ vs 10 $\mathrm{m} \mathrm{s}^{-1}$ ) (Figs. 4.3.2c,f). The LLJ descends towards the surface during the night as the boundary-layer depth decreases (Fig. 4.3.2e) leading to a minimum jet height of $300 \mathrm{~m}$ and $200 \mathrm{~m}$ for the urban and the rural sites, respectively. A similar response is observed during the second night, but the descent is smaller $(50-100 \mathrm{~m})$. The difference in the descent of the jet is likely to be caused by the larger decrease of nocturnal mixed-layer height during the first night compared to the second night. The jet speed decreases by 2-3 $\mathrm{m} \mathrm{s}^{-1}$ for the urban site $\left(4-5 \mathrm{~m} \mathrm{~s}^{-1}\right.$ for the rural one) during the first night (Figs. 4.3.2e,f), but this should be handled with care since there is missing data near the jet peak. No clear decrease in jet speed is observed during the second night (Figs. 4.3.2e,f). The decrease in LLJ speed during the first night is related to the large-scale momentum advection in the hours prior to the formation of the LLJ, which results in an immediate formation of a strong LLJ (without intensification phase). This is evident from the peak in wind speed at 2000 UTC in Figs. 4.3.2a,c, which is driven by baroclinicity associated with the strong north-south pressure gradient over the North Sea (Fig. A.4.1). On the contrary, during the second night baroclinicity is weaker (Fig. A.4.2) and the LLJ exhibits an intensification phase more closely associated with an IO, with wind speeds increasing after sunset (Figs. 4.3.1b,d). This is evident in WRF but it is not easily identified from the Doppler Lidar due to larger variability in the measured wind speeds.

\subsubsection{Evaluation of WRF and UKV with observations}

In this section we evaluate the modelled LLJ characteristics (fall-off, height and speed) using the Doppler Lidars at the urban (Southbank, London) and rural (Chilbolton) sites.

Both models (WRF and UKV) are able to accurately reproduce the LLJs during the 

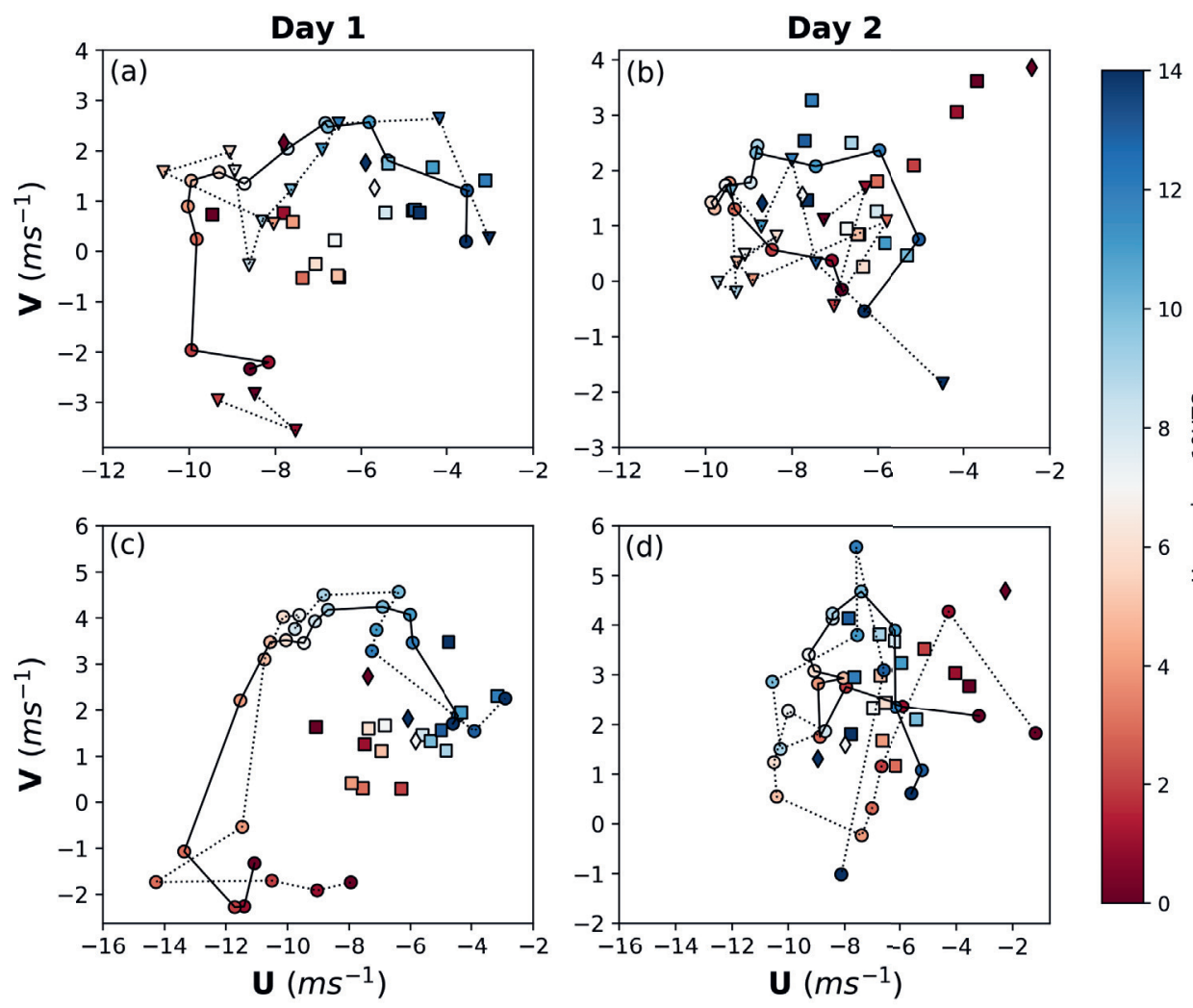

$$
\text { — WRF } \quad \text { W WRF_geowind } \diamond \text { ECMWF_geowind ….... LIDAR }
$$

Figure 4.3.1: Evolution of modelled (WRF) and observed (Doppler Lidar) $\mathrm{u}$ and $\mathrm{v}$ wind components and $u, v$ geostrophic wind components (WRF, ECMWF) during the first (a,c) and second (b,d) night of the case study period for Southbank (a,b) (400 m a.g.l) and Chilbolton (c,d) (300 m a.g.l). Hourly U and V wind components (and geostrophic wind from WRF) are used starting at 1900 UTC 14 May 2019 (1 hour before sunset) until 1000 UTC for both nights. Geostrophic wind components from ECMWF are available every 6 hours (at 1800, 0000 and 0600 UTC).

case study period (Fig. 4.3.2). The formation and dissipation of the LLJs in WRF and UKV occur one hour earlier than observed (2300 UTC 14 May 2019) over London and Chilbolton during the first night. During the second night, the LLJ is formed 2 hours earlier than observed in UKV and WRF (at 2100 UTC 15 May 2019). Both models are able to capture the decrease in LLJ height over London during the first night. However, only a small decrease in the LLJ height is reported over the rural site during the first night, while during the second night the LLJ height remains rather constant. 

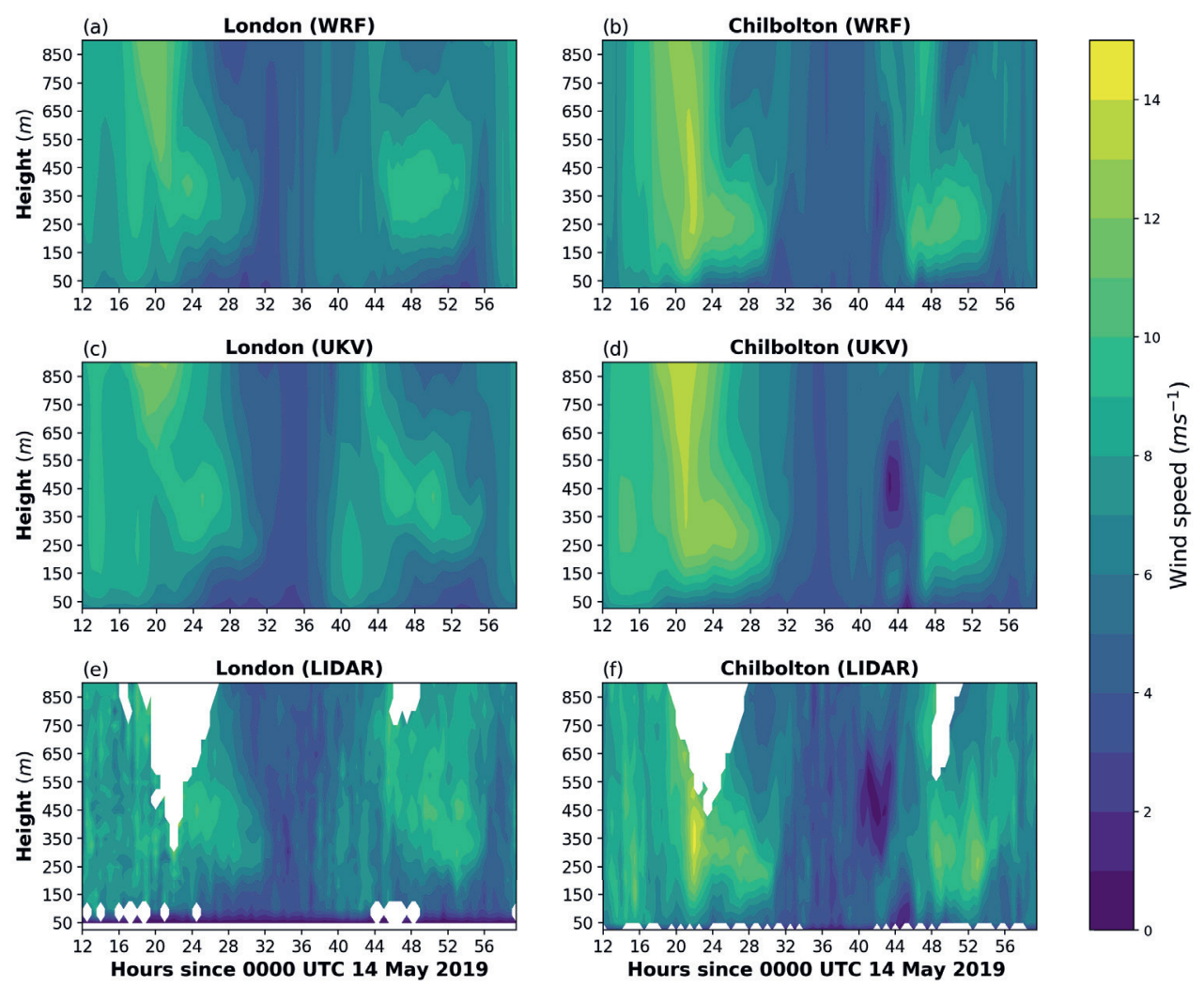

Figure 4.3.2: Modelled (WRF,UKV) and observed (Lidar) wind speed $\left(\mathrm{m} \mathrm{s}^{-1}\right)$ profile evolution over time for the two locations London (LSU) $(\mathrm{a}, \mathrm{c}, \mathrm{e})$ and Chilbolton $(\mathrm{b}, \mathrm{d}, \mathrm{f})$ for the period 1200 UTC $14 / 05 / 2019$ to 1200 UTC 16/05/2019. Areas with large wind speed error estimate $\left(>2 \mathrm{~m} \mathrm{~s}^{-1}\right)$ are masked (white). Doppler Lidar wind speed values are half-hourly averages, while WRF and UKV use half-hourly and hourly instantaneous values respectively.

The initial jet speed over Southbank is captured well in WRF and UKV for both nights. However the bias in the modelled jet speed increases over time, because both models tend to dilute the LLJ faster than observed. For the first night a lower $v_{g}$ (v-component of the geostrophic wind) in the WRF model (1 vs $2 \mathrm{~m} \mathrm{~s}^{-1}$ compared to the 6-hourly ECMWF operational data) leads to a larger ageostrophic v-component ( $\left.\mathrm{v}-v_{\text {geo }}\right)$, which could have lead to a faster reduction of the u-wind component. During the second night, the modelled LLJ evolution leads the observations by 2 hours. This results to faster decay of the modelled jet compared to the observations and consequently an increase in negative bias over time. This resulted in a mean jet speed bias of $-0.72 \mathrm{~m} \mathrm{~s}^{-1}$ (WRF) and $-0.60 \mathrm{~m} \mathrm{~s}^{-1}$ in the UKV during the two nights (Table 4.3.1). Over Chilbolton, the LLJ speed mean bias is larger in $\operatorname{WRF}\left(-0.61 \mathrm{~m} \mathrm{~s}^{-1}\right)$ than in the $\operatorname{UKV}\left(-0.35 \mathrm{~m} \mathrm{~s}^{-1}\right)$. Overall, 

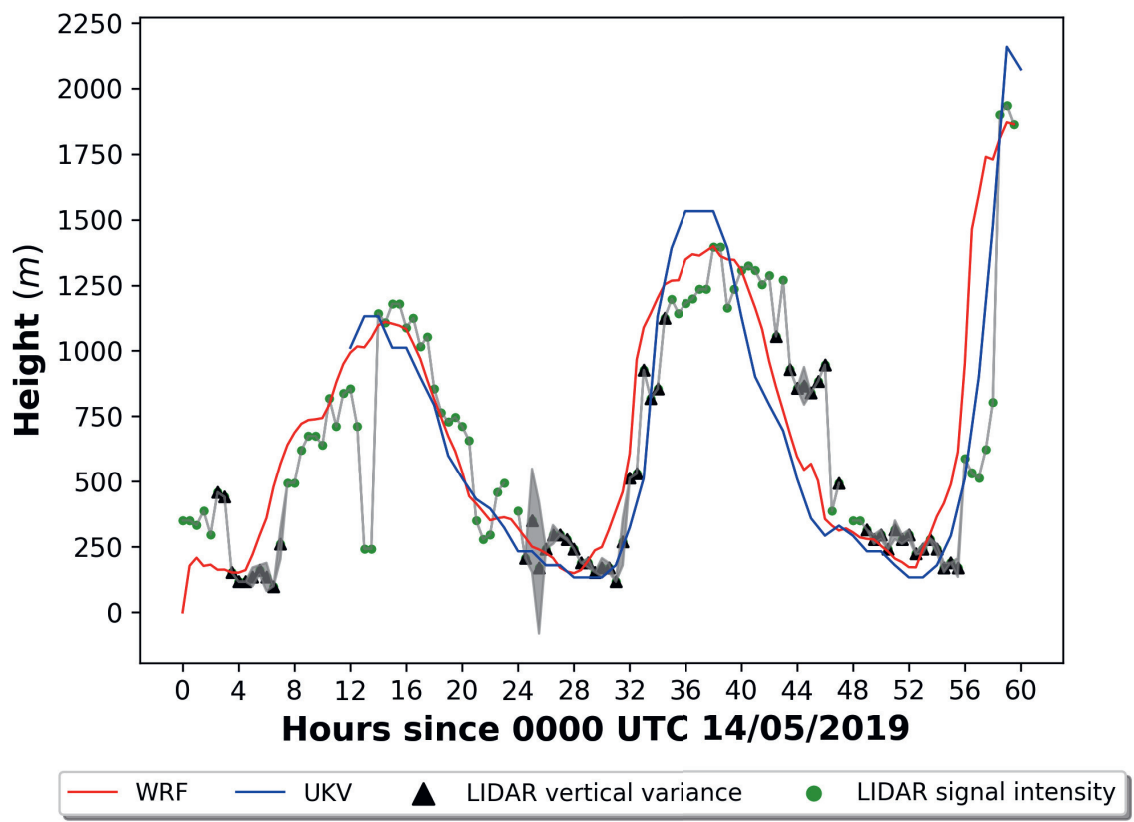

Figure 4.3.3: Boundary-layer height (m) over Southbank, London derived from WRF (red), UKV (blue) and the Doppler Lidar (black triangles for the $50^{\text {th }}$ percentile of the vertical variance method, green dots for the signal intensity method) for the period 0000 UTC 14 May 2019 to 1200 UTC 16 May 2019 .The shaded gray area indicates uncertainty range $\left(75^{t h}\right.$ to $25^{\text {th }}$ percentile) from the Lidar-derived mixed-layer height when the vertical velocity variance profiles is used. Detail information on the Lidar-derived mixed-layer height are provided in section 4.2.4.

both models show very similar performance. It is difficult to concretely conclude whether one of the two models is better at capturing the LLJ speed and height, considering that there are no measurements of wind speed during the initial phase LLJ during the first night.

The LLJ height is substantially underestimated over London (-57.3 $\mathrm{m}$ in WRF and -58.8 in UKV) (Table 4.3.1). This is caused by an underestimation of the UBL height during the initial phases of the LLJ formation (2200-2400 the first night and 2000-2300 UTC the second night), as the large initial bias in LLJ and UBL heights decreases over time (Fig. 4.3.3). This is also evident by the observed kinematic heat flux measured at the BT tower (175 m a.g.l, site details at Wood et al. (2010)), which is generally higher than both WRF and UKV from 2000 UTC until 0000 UTC during both nights and causes a delay in the collapse of the observed UBL, especially during the second night (Figure A.4.3). It is possible that the lower LLJ height in the two models inhibits the intensification of 
the LLJ and thus contributes to the negative bias in jet speed. This effect would be more profound during the first night, where the LLJ caused by large-scale momentum advection, as the imbalance between the actual and geostrophic wind is lower closer to the surface due to stronger turbulent mixing. The bias in modelled LLJ height over Chilbolton is substantially lower (-25.0 $\mathrm{m}$ in WRF and $-8.8 \mathrm{~m}$ in UKV) than over London.

The LLJ fall-off is overestimated over London $\left(0.46 \mathrm{~m} \mathrm{~s}^{-1}\right.$ in WRF vs $0.05 \mathrm{~m} \mathrm{~s}^{-1}$ in UKV), but underestimated over Chilbolton $\left(-0.54 \mathrm{~m} \mathrm{~s}^{-1}\right.$ in WRF and $-0.60 \mathrm{~m} \mathrm{~s}^{-1}$ in UKV) during both nights. During the second night, the positive bias in fall-off over the urban site mainly originates from a strong negative bias in upper residual-layer wind speed (Fig. 4.3.2) during the second night. WRF also shows a negative bias during the first night, which is caused by the advection of a low-wind-speed air mass between 700-9000 $\mathrm{m}$ around 2000 UTC. Over Chilbolton the underestimation in LLJ fall-off is caused by a positive bias in the $700-900 \mathrm{~m}$ wind speed in both models.

Table 4.3.1: Model bias (WRF and UKV) for LLJ speed $\left(\mathrm{m} \mathrm{s}^{-1}\right)$, height $(\mathrm{m})$ and falloff $\left(\mathrm{m} \mathrm{s}^{-1}\right)$ of the LLJs in comparison to the in-situ Doppler Lidars at London (LSU) and Chilbolton. Best score per site and metric is highlighted (bold). Statistical scores is an average of the hourly biases both nights and calculated only at times when an LLJ is detected in the Doppler Lidar profiles.

\begin{tabular}{ccccc}
\hline Site & \multicolumn{2}{c}{ London } & \multicolumn{2}{c}{ Chilbolton } \\
\hline Model & WRF & UKV & WRF & UKV \\
\hline Peak speed & -0.72 & $\mathbf{- 0 . 6 1}$ & -0.61 & $\mathbf{- 0 . 3 5}$ \\
Height & $\mathbf{- 5 7 . 3}$ & -58.8 & -25.0 & $\mathbf{- 8 . 8}$ \\
Falloff & 0.46 & $\mathbf{0 . 0 5}$ & $\mathbf{- 0 . 5 4}$ & -0.60 \\
\hline
\end{tabular}

\subsection{Differences in LLJ characteristics between Lon- don and Chilbolton}

In this section we analyze the differences in the LLJ occurrence frequency and characteristics between London and Chilbolton obtained from the Doppler Lidar observations and the two NWP models for two periods : a) 1900 UTC 14 May 2019 to 1000 UTC 15 May 2019 and b) 19 UTC 15 May 2019 to 10 UTC 16 May 2019. This is done by applying the LLJ detection algorithm (section 4.2.5) to the $30 \mathrm{~min}$ wind speed obtained from the WRF model and the Doppler Lidar, and the hourly wind speed from the UKV (Fig. 4.4.1). For WRF and UKV data we quantify the LLJ characteristics at each time step and over all urban grid-cells of London within a $75 \times 60 \mathrm{~km}$ area, for which an LLJ is detected. The LLJ characteristics over Chilbolton are quantified in an area of $45 \times 45 \mathrm{~km}$. This allows the incorporation of the spatial variability in LLJ characteristics and makes the comparison between urban and rural LLJ characteristics more robust. The observed 
occurrence frequency of the LLJs is very similar for both Southbank (34\%) and Chilbolton $(32 \%)$. A non-parametric Mann-Whitney test is also conducted to tests for statistical significance of the differences in LLJ characteristics between the two sites. Since the data from WRF and UKV for both sites have the potential to be spatially auto-correlated (i.e. reduced effective sample size), we used a randomly selected sample of $5 \%$ from all the quantified model data the Mann-Whitney test. The test is iterated 100 times to get a robust statistical significance.

The LLJs are located at higher heights over London, with the observed LLJ height ranging between 200-550 m, with a mean LLJ height of $322 \mathrm{~m}$ for the first night and $352 \mathrm{~m}$ for the second night (Fig. 4.4.1c,d). Over Chilbolton the LLJ height ranges from 150-425m, with mean LLJ heights of $267 \mathrm{~m}$ (first night) and $293 \mathrm{~m}$ (second night). Similar differences are also seen in the UKV model with the LLJ height ranging between 150-500m over London and an average LLJ height of $305 \mathrm{~m}$ and $349 \mathrm{~m}$ both for the first and second night, respectively. Over Chilbolton the LLJ height ranges between 150-450m in UKV (peak frequency at $275 \mathrm{~m}$ the first night and a $325 \mathrm{~m}$ peak during the second night). The WRF model shows a similar range in the distribution to the UKV, but the mean LLJ height is slightly lower at $304 \mathrm{~m}$ (first night) and $325 \mathrm{~m}$ (second night), while over the rural site the mean LLJ is $233 \mathrm{~m}$ for both nights. The difference in LLJ height between London and Chilbolton is statistically significant $(\mathrm{p}<0.05)$ for both WRF and UKV. For the LLJ detected by the Doppler Lidar the differences in LLJ height are statistically significant for the second night $(p=0.036)$ but not for the first $(p=0.055)$. However these tests should be handled with care as the uncertainty of the measured wind speed profile, especially for the first night, could affect the probability distribution. The LLJ height differences can be partially attributed to the deeper boundary layer over London compared to Chilbolton (see section 4.6.

Table 4.4.1: Mean values and standard deviation for LLJ speed $\left(\mathrm{m} \mathrm{s}^{-1}\right)$, height $(\mathrm{m})$ and falloff $\left(\mathrm{m} \mathrm{s}^{-1}\right)$ over London and Chilbolton, from the Doppler Lidar and the WRF and UKV models for the first (1900UTC 14/05/2020 - 1000 UTC 15/05/2020) and second (1900 UTC 15/05/2020 - 1000 UTC 16/05/2020) night.

\begin{tabular}{ccccccc}
\hline Site & \multicolumn{3}{c}{ London } & \multicolumn{3}{c}{ Chilbolton } \\
& WRF & UKV & Doppler Lidar & WRF & UKV & Doppler Lidar \\
\hline (a) First night & & & & & \\
Height (std) & $304(64)$ & $305(63)$ & $322(115)$ & $235(61)$ & $261(58)$ & $267(108)$ \\
Speed (std) & $8.5(1.4)$ & $8.5(1.5)$ & $7.9(2.2)$ & $10.7(1.4)$ & $11.1(1.7)$ & $9.4(3.2)$ \\
Falloff (std) & $3.5(1.0)$ & $2.3(0.5)$ & $2.7(1.1)$ & $3.6(1.1)$ & $2.9(0.7)$ & $3.7(2.1)$ \\
\hline (b) Second night & & & & & \\
Height (std) & $325(62)$ & $349(79)$ & $359(126)$ & $232(86)$ & $273(86)$ & $293(115)$ \\
Speed (std) & $9.4(0.7)$ & $9.0(0.9)$ & $8.9(2.4)$ & $9.7(1.1)$ & $8.6(1.7)$ & $8.5(2.6)$ \\
Falloff (std) & $3.6(1.2)$ & $3.8(1.2)$ & $2.0(0.73)$ & $3.6(1.4)$ & $3.6(1.3)$ & $3.3(1.1)$ \\
\hline
\end{tabular}

The observed LLJ speed is higher over Chilbolton $\left(9.4 \mathrm{~m} \mathrm{~s}^{-1}\right)$ with the frequency dis- 

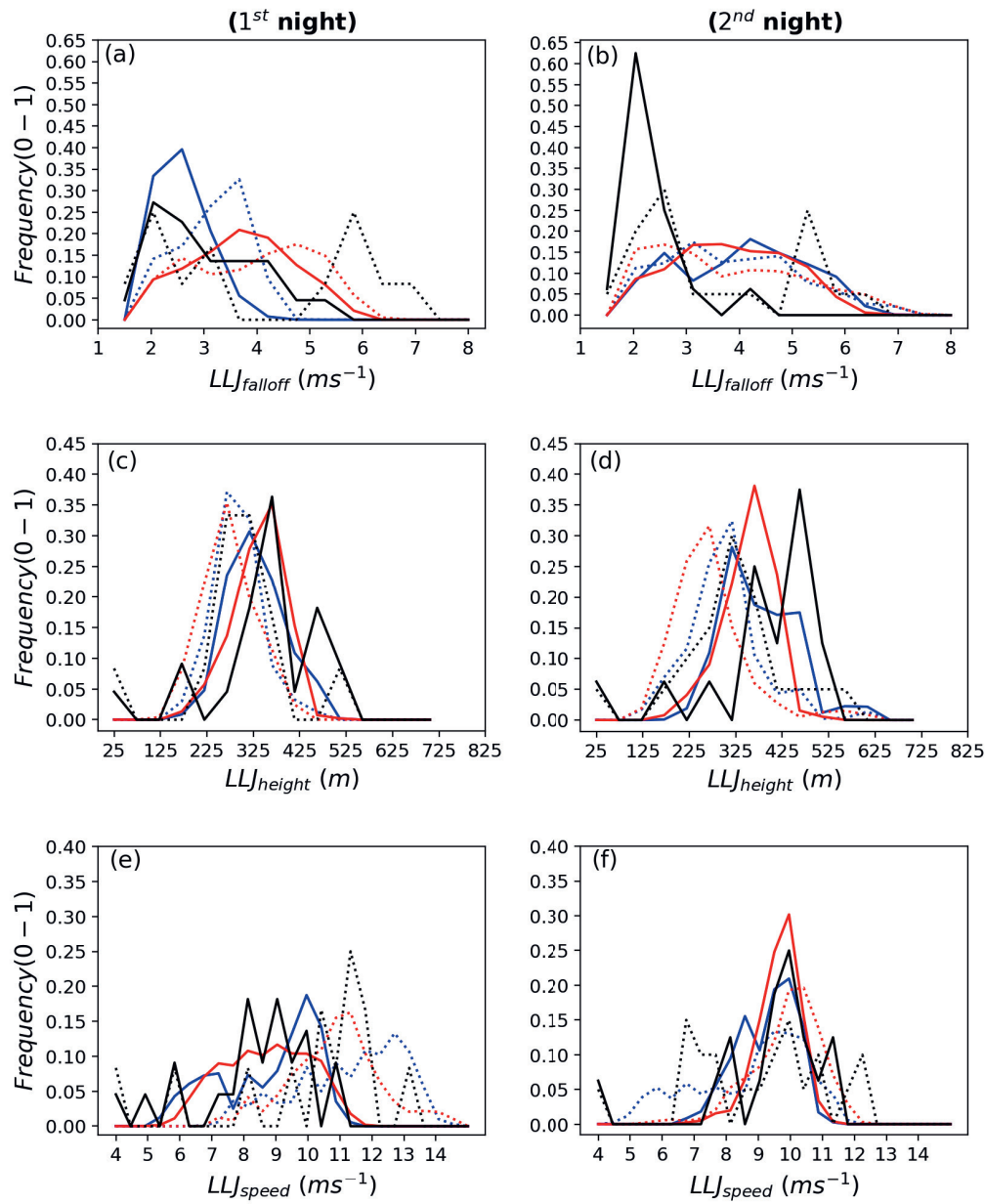

\begin{tabular}{|c|c|c|c|c|c|}
\hline- & London_UKV & - & London_WRF & $\overline{\ldots \ldots}$ & London_LIDAR \\
\hline
\end{tabular}

Figure 4.4.1: Modeled (red for WRF, blue for UKV) and observed (black) frequency distribution of the LLJ fall-off $(\mathrm{a}, \mathrm{b})$, height $(\mathrm{c}, \mathrm{d})$ and speed $(\mathrm{e}, \mathrm{f})$ for London (solid lines) and Chilbolton (dotted lines) during the first (1900 UTC 14/05/2019 to 1000 UTC 15/05/2019) and second period (1900 UTC 15/05/2019 to 1100 UTC 16/05/2019).

tribution shifted towards higher wind speeds compared to London $\left(7.9 \mathrm{~m} \mathrm{~s}^{-1}, \mathrm{p}<0.05\right)$ during the first night (Fig. 4.4.1, Table 4.4.1). However, this difference is reversed during the second night with the urban LLJ being $0.4 \mathrm{~m} \mathrm{~s}^{-1}$ faster (8.9 vs $8.5 \mathrm{~m} \mathrm{~s}^{-1}, \mathrm{p}>0.05$ ), but this result is not statistically significant. This indicates that the difference in LLJ speed between the two sites is probably affected by non-urban effects, such as differences 
in geostrophic wind and large-scale momentum advection. Both WRF and the UKV show similar differences in the LLJ speed between the two sites as the Doppler Lidar observations (Table 4.4.1), which are statistically significant $(\mathrm{p}<0.01)$, but WRF still maintains a slightly lower LLJ speed over London during the second night.

The average observed LLJ fall-off over Chilbolton is $3.7 \mathrm{~m} \mathrm{~s}^{-1}$ and $3.3 \mathrm{~m} \mathrm{~s}^{-1}$ during the two nights (Fig. 4.4.1a,b and Table 4.4.1). Over London the fall-off is 2.7 and 2.0 $\mathrm{m} \mathrm{s}^{-1}$, during the first and second night, respectively. The difference in the observed fall-off are statistically significant for the second night, but not the first night. The bimodal distribution over Chilbolton is likely to be caused by the lack of observed wind above $500 \mathrm{~m}$ during the initial phases of the LLJ formation (especially during the 14th May 2019), which results in the identification of smaller fall-offs by the detection algorithm and limits the value of the significance test for the first night. This is also the case for the LLJ over London during the first night, but not during the second night. The differences in the fall-off distribution between the London and Chilbolton can be attributed to differences in the LLJ speed between the two sites during the first night and lower 700-900m wind speed over Chilbolton the second night (Fig. 4.4.1). The WRF model shows small differences between urban and rural fall-off only during the second night (Table 4.4.1), but they are not statistically significant. The difference in the urban and rural fall-off in the UKV data are statistically significant for both nights. The difference in the LLJ fall-off distributions between UKV and WRF during the first night is attributed to a the lower wind speed at 700-900m in the WRF model (Figs. 4.3.2 and 4.4.1a). This highlights the sensitivity of fall-off to large-scale momentum advection and the accurate representation of geostrophic wind speed. 


\subsection{Spatial distribution of LLJ characteristics over London}

Here we investigate the spatial distribution of the LLJ characteristics over London and the surrounding rural areas using both the WRF and the UKV model. This allows us to better identify differences in the spatial distribution of the LLJ characteristics that might originate from non-urban effects (i.e. topography) and to investigate the intraurban variability in LLJ characteristics. This analysis is conducted in a $75 \times 60 \mathrm{~km}$ area centred around London, so it excludes the rural site of Chilbolton. The predominant wind direction at $850 \mathrm{hPa}$ is easterly during both nights, but can vary slightly from east-northeast to east-south-east during the course of the night, especially closer to the surface (Figs. A.4.1,A.4.2).

The LLJ height is generally higher over the urban area (i.e. urban grid-cells), compared to the countryside around London (Figs. 4.5.1c,d and 4.5.2c,d). In the WRF model the LLJ height difference between urban and rural grid-cells, within the $60 \times 75 \mathrm{~km}$ area, is approximately $50 \mathrm{~m}$ during both nights, while in the UKV it is $47 \mathrm{~m}$ for both nights. Part of this difference can be attributed to the increased boundary-layer height over London, an effect of the different energy balance and roughness of the urban surface. As stated in Klein et al. (2016) and Pichugina \& Banta (2010), the LLJ nose is usually a located above the stable boundary layer, where there is a local minimum in the vertical mixing. The deeper UBL increases the height of this local minimum in the vertical mixing, thus increasing the height at which the LLJ is located. Similarly, the urban plume increases the UBL depth and vertical mixing downwind of the urban area leading to elevated LLJ heights over the downwind rural areas in both models (Fig. 4.5.1c,d, Sect. 4.6), but since they are located over rural grid-cells they do not contribute to the aforementioned height differences in Figure 4.4.1. Overall, the modelled LLJ height is positively correlated with the modelled boundary-layer depth $(\mathrm{r}=0.80, \mathrm{p}<0.001$ for the first night; $\mathrm{r}=0.78, \mathrm{p}<0.001$ for the second night). Yet as discussed in section 4.6 the terrain height influences the LLJ height $(\mathrm{r}=-0.82, \mathrm{p}<0.001$ for the first night; $\mathrm{r}=-0.68, \mathrm{p}<0.001$ for the second night). The impact of topography is also evident in elevated LLJ height upwind of London. Here, the steep topographic features near the coast (Fig. 4.2.1c) result in a channelling of flow from the North Sea into the Thames Valley. This results in the transport of the elevated LLJ, which is present over the Thames estuary, upwind through the Thames valley over London.

The difference in LLJ speed between London and the rural surroundings is minimal for the first night $\left(0.27 \mathrm{~m} \mathrm{~s}^{-1}\right.$ in UKV, $0.15 \mathrm{~m} \mathrm{~s}^{-1}$ in WRF $)$ and non-existent the second night $\left(<0.05 \mathrm{~m} \mathrm{~s}^{-1}\right)$. An increase in LLJ speed around the edges of the city (south and north-east) is visible (Figs 4.5.1,e,f). There is also a general increase of the LLJ speed towards the south, which is associated with stronger geostrophic wind and larger advection 

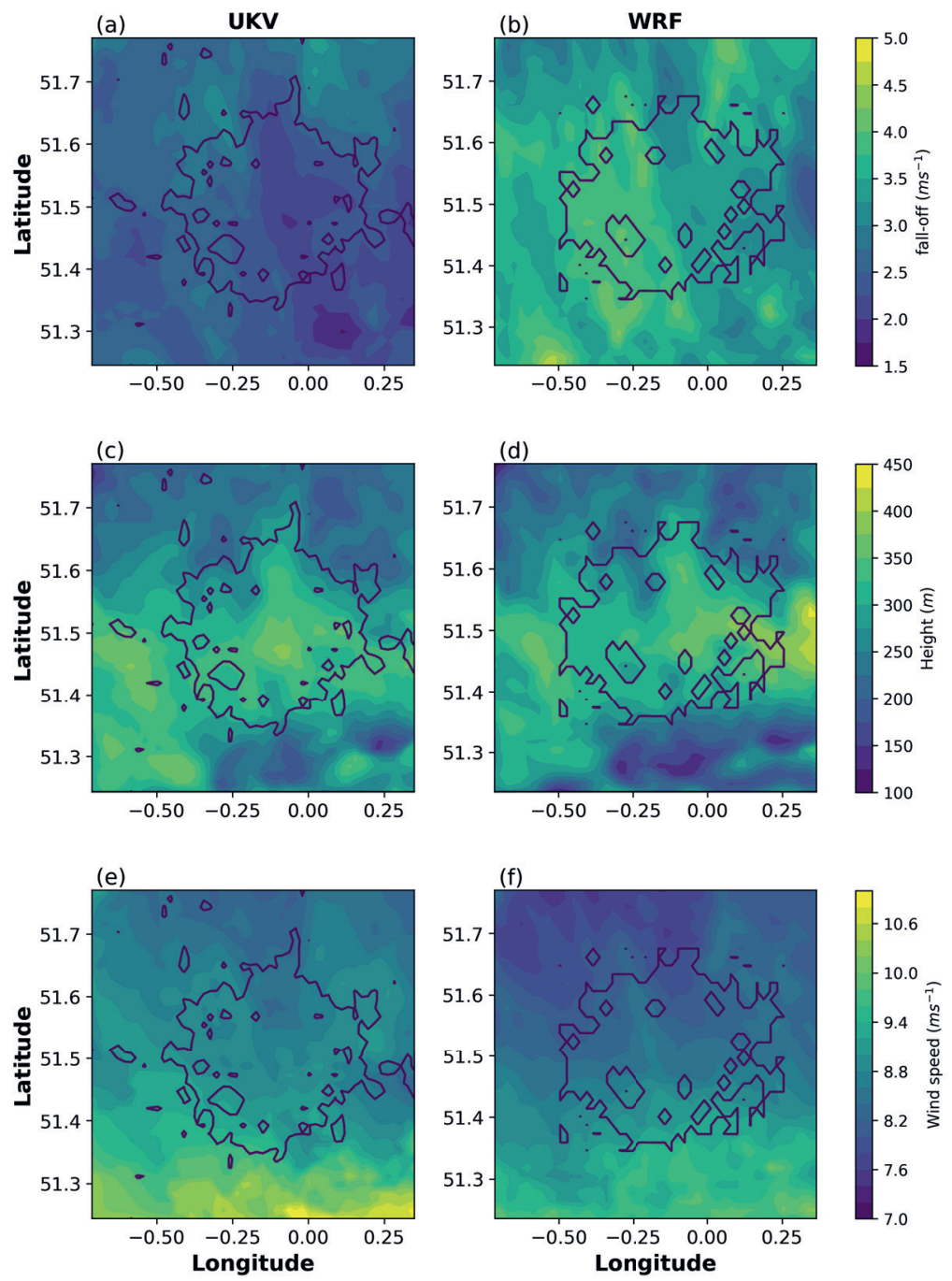

Figure 4.5.1: Spatial distribution of the time-averaged LLJ fall-off (a,b), height $(\mathrm{c}, \mathrm{d})$ and peak speed $(\mathrm{e}, \mathrm{f})$ within and around London in the UKV (a,c,e) and the WRF (b,d,f) models, during the first night (2100 UTC $14 / 05 / 2019$ to 0600 UTC 15/05/2019). The boundary of London's urban area are define (black line) for both WRF (land use grid-cell index is urban) and UKV (urban fraction $>0.4$ ).

of momentum due to stronger baroclinicity south of London (Figs. 4.5.1,e,f and A.4.1, A.4.3). No direct correlation exist with either topography or boundary-layer height and the LLJ speed. 

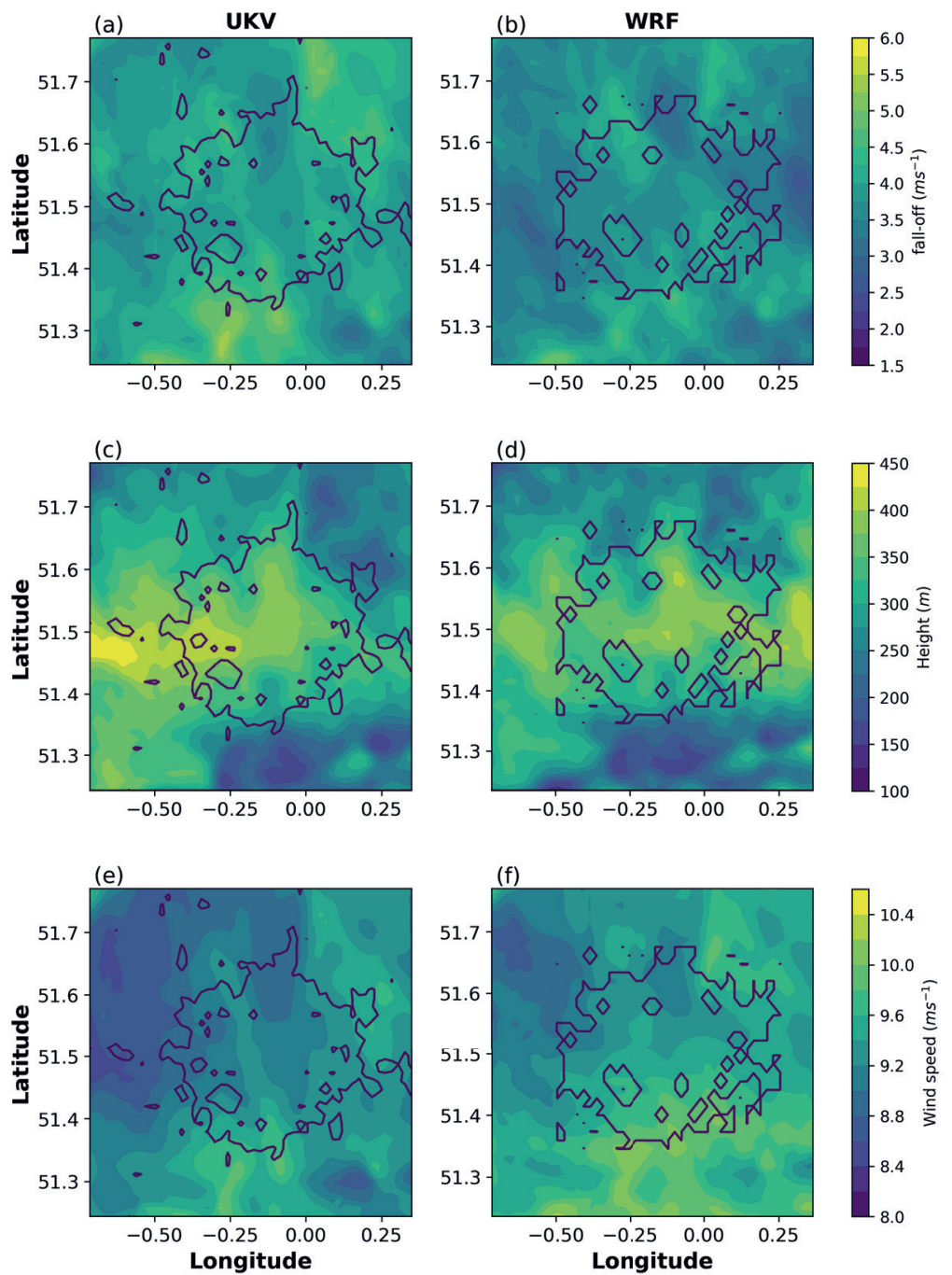

Figure 4.5.2: As Figure 4, but for the second night (2100 UTC 15/05/2019 to 0600 UTC 16/05/2019)

Considering the spatial variability in LLJ characteristics, we interestingly find an anticorrelation between the LLJ height and speed (Figs. 4.5.1c,d,e,f). A Spearman correlation-coefficient test between LLJ height and LLJ speed showed no $(\mathrm{r}=0.05$, $\mathrm{p}=0.042)$ to little $(\mathrm{r}=-0.38, \mathrm{p}<0.001)$ anti-correlation during the first and the second night. However, the jet speed does decrease during the transition from model gridcells with lower LLJ height to grid-cells with higher LLJ heights and vice versa (Figs. 
4.5.1c,d,e,f). To test this hypothesis Spearman correlation coefficient tests between the derivatives of the WRF derived LLJ speed $\left(d U_{L L J} / d x\right)$ and height $\left(d z_{L L J} / d x\right)$ across the $\mathrm{x}$ (west-east) direction for the first $(\mathrm{r}=-0.66, \mathrm{p}<0.001)$ and second night $(\mathrm{r}=-0.78$, $\mathrm{p}<0.001)$ were conducted. A similar, yet weaker, anti-correlation is reported along the $\mathrm{y}$ (south-north) direction for the first $(\mathrm{r}=-0.48, \mathrm{p}<0.001)$ and second night $(\mathrm{r}=-0.50$, $\mathrm{p}<0.001$ ). However, this is to be expected due to the increase in the geostrophic towards the south (especially during the first day), which might be more relevant for the LLJ speed than the change in the LLJ height the y direction. This anti-correlation across the west-east direction is also seen in the modelled LLJ height and speed in the UKV model ( $\mathrm{r}=-0.38$ for the first night and $\mathrm{r}=-0.79$ during the second night) and also exist in a similar spatial analysis for Chilbolton $(r=-0.39$ for the first night and $r=-0.49$ the second night with $\mathrm{p}<0.001$ for both nights).

The spatial variability of LLJ fall-off (Figs. 4.5.1a,b) can be partially explained by the changes in the spatial distribution of the LLJ speed over the domain, assuming that wind in the upper part of the residual layer does not change drastically over London during the night (i.e. closely follows the geostrophic wind). However, since we already showed that the geostrophic wind increases towards the south, the spatial variability in LLJ falloff cannot be solely based on the fluctuations of the LLJ speed over the domain. The relation between the spatial variability in LLJ height, speed and fall-off is further analysed in section 4.6 and a discussion of possible mechanisms that could explain it is provided in section 4.8 .

\subsection{Isolating urban and non-urban effects on LLJ characteristics}

In this section, we conduct a series of idealized sensitivity experiments using the WRF "reference" setup (section 4.2.2) to isolate the influence of non-urban (i.e. topographic) effects on the difference in the LLJ occurrence frequency and characteristics between the two sites. The experiments include : a) replacement of the urban LU classes of London with the dominant surrounding vegetation (cropland) and b) removal of topographic features (terrain height set to $0 \mathrm{~m}$ ) in the inner WRF domain. As in the previous LondonChilbolton comparison (Sect. 4.4) the differences are calculate for all grid-cells designated as urban in the reference experiment over the designated $75 \times 60 \mathrm{~km}$ area around London. This is done to allow a direct comparison between the two sections. Differences in LLJ characteristics that occur in non-urban grid-cells are still discussed but their magnitudes is not included in the mentioned LLJ height, speed and fall-off differences. 


\subsubsection{Urban effects on the LLJ characteristics over London}

In the first idealized experiment the urban area of London is replaced by cropland (the dominant surrounding vegetation). It is worth mentioning that the LLJ characteristics over the Chilbolton site are virtually the same in the cropland and reference experiments ( $<3 \mathrm{~m}$ difference in LLJ height, $<0.1 \mathrm{~m}$ difference in LLJ speed).

The frequency distribution (Figs. 4.6.1c,d) indicates that the LLJ heights decrease (150 - $450 \mathrm{~m}$ ), with a mean LLJ height of $275 \mathrm{~m}$ during the first night and $282 \mathrm{~m}$ for the second night. Compared to the reference run, the cropland run shows a decrease in LLJ heights during the first $(34 \mathrm{~m})$ and the second $(43 \mathrm{~m})$ night. These differences are statistically significant $(\mathrm{p}<0.001)$. The differences in LLJ height are also evident in the spatially distribution of LLJ height within and around London (Figs. 4.6.2d,e). A possible mechanism that explains the higher LLJ height in the reference run is that the deeper nocturnal UBL, due to more vertical mixing, increase the height at which the turbulent drag impacts the wind flow and thus inhibits the formation strong IO closer to the surface. This dependency of LLJ height to vertical mixing intensity is also reported by Klein et al. (2016), has been observed during the Joint Urban 2003 campaign (Wang et al., 2007) and would be consistent with the finding that the LLJ nose usually occurs above the top of the nocturnal boundary layer Van de Wiel et al. (2010); Pichugina \& Banta (2010).

The LLJ speed is slightly higher in the cropland experiment, with a distribution shifted towards higher wind speeds (Figs. 4.6.1,4.6.2e,f). During both nights the mean LLJ speed is $0.25 \mathrm{~m} \mathrm{~s}^{-1}$ higher when the urban area is removed. However, the results are significant during both nights $(\mathrm{p}<0.05)$, but with much larger $\mathrm{p}$ value $(\mathrm{p}<0.001)$ during the second night. During the first night the large spread of the LLJ speeds increases the standard deviation making the difference less significant. Consequently, the LLJ fall-off increases slightly $\left(0.11 \mathrm{~ms}^{-1}\right)$ in the cropland run, which is likely to be caused by the increase in LLJ speed (as discussed in section 4.5).

It is also worth noticing that the increase in LLJ height and decrease in LLJ speed occurs mainly downwind (west-north-west) of London (Figs. 4.6.2c,e,), which matches the finding of Wang et al. (2007) and Lemonsu et al. (2009) on the impact of Oklahoma City on the LLJ downwind of the urban area. The differences in LLJ height and speed, between the reference and the cropland runs, show a strong anti-correlation $(\mathrm{r}=-0.73, \mathrm{p}<0.001)$ during both nights. This supports our previous analysis that showed a decrease in LLJ speed when LLJ height increases, and highlights their dependency on the boundary-layer depth and the vertical mixing within the nocturnal UBL.

In the cropland experiment, the LLJ is initialized an hour earlier over London during both nights compared to the reference experiment. Moreover the LLJ is detected approximately $15 \mathrm{~km}$ further downwind by $23 \mathrm{UTC}$ compared to the reference experiment during both 

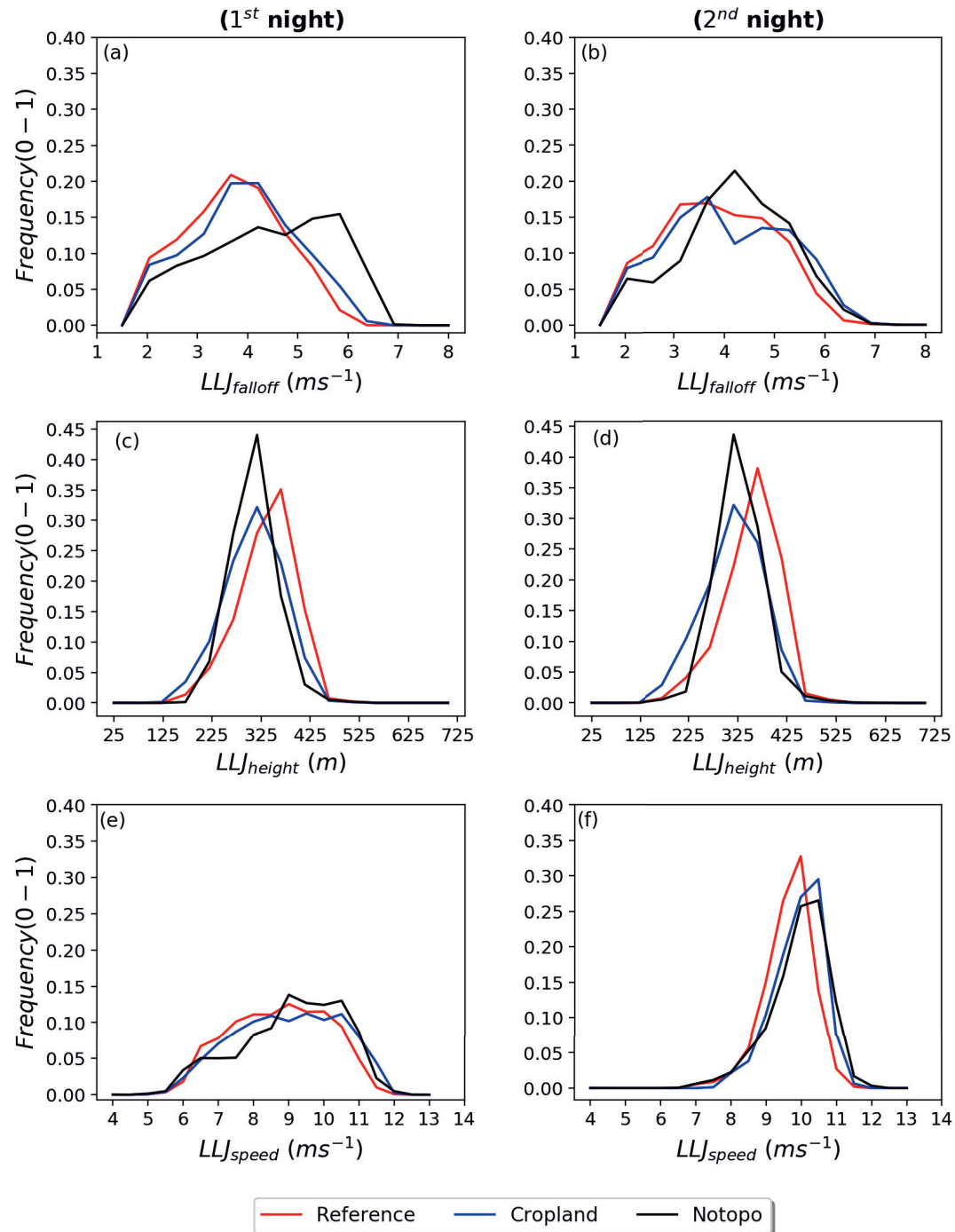

Figure 4.6.1: Frequency distribution of LLJ characteristics (fall-off, height and peak speed of LLJ) over London for the reference (a,d,g), cropland (b,e,h) and notopo (c,f,i) experiments during the first (1800 UTC 14/05/2019 to 1100 UTC 15/05/2019) and second nights (1800 UTC $15 / 05 / 2019$ to 1100 UTC $16 / 05 / 2019)$ of the case study

nights. This indicates that the instability and the vertical turbulent mixing still present in the nocturnal UBL during the evening (1800-2200 UTC) delays the de-coupling of the flow and could potentially decrease the initial acceleration phase due to the IO, thus also delaying the inland propagation of the LLJ (during the first night). The faster onset of 
LLJ over the now rural London highlights the potential of LLJ being advected over the urban area from the surrounding rural areas as proposed by Barlow (2014).

It is evident that the different LLJ heights over London between the cropland and the reference experiments ( $33 \mathrm{~m}$ for the $1^{\text {st }}$ night, $43 \mathrm{~m}$ for the $2^{\text {nd }}$ night respectively), do not match the differences in LLJ height between London and Chilbolton (69 $\mathrm{m}$ and 90 $\mathrm{m}$ respectively) (Figs. 4.4.1,4.6.1). This indicates that the differences between London and Chilbolton are not cause solely by the urban area of London. Based on this analysis the impact of the urban area could be contributing approximately $50 \%$ of the reported LLJ height difference between the two sites (Sect. 4.4). Moreover, impact of the urban area on the LLJ speed over London is small $\left(0.25 \mathrm{~m} \mathrm{~s}^{-1}\right.$ lower LLJ speed in the reference experiment) compared to the difference reported between the two sites for the first night $\left(2.1 \mathrm{~ms}^{-1}\right)$.

\subsubsection{Topographic effects on the LLJ characteristics over London}

Here the reference experiment is compared to an idealized experiment where terrain height in the inner WRF domain is set to zero (notopo experiment).

Although the spread of the LLJ heights distribution remains the same (125-450m) the mean LLJ height decreases over the urban area by approximately $35 \mathrm{~m}$ compared to the reference experiment (Figs. 4.6.1c,d). To explain these difference we investigate the spatial distribution of the LLJ height (Fig. 4.6.2d). In the notopo experiment the LLJ height over and upwind of London is decreased (Fig. 4.6.2d), but this cannot be explained by the changes in boundary-layer depth between the two experiments. Instead it is explained by the absence of the higher LLJ heights over the Thames estuary, which is present in the reference run, but does not exist in the notopo experiment. Therefore, there is no channelling of elevated LLJs over London in the notopo experiment, which results in generally lower LLJ height over London. The elevated LLJ over the Thames estuary is probably caused by orographic drag of the nearby topography on the air flow from the North Sea is the reference run, as it is not present when the topography is removed. The lack of flow channelling and the deeper boundary layers north and south of London result in an increase of the LLJ height over these areas in the notopo experiment Fig. 4.6.2d).

The removal of topographic features also increases the modelled LLJ speed over London (Fig. 4.5.1f). In the notopo run the spread of the LLJ speed frequency distribution increase by $1 \mathrm{~m} \mathrm{~s}^{-1}$ (Figs. 4.6.1e,f), but the mean LLJ speed increases only by 0.3 $\mathrm{m} \mathrm{s}^{-1}$ (both nights). A larger increase in LLJ speed occurs south of London, when the topography is removed. This an effect of the increased wind speed due to the lack orographic drag in the notopo experiment. The increase in LLJ speed also causes an increase of the LLJ fall-off when the topography is removed (Figs. 4.6.1a,b). However, the fact that the LLJ fall-off increases more than the LLJ speed in the notopo experiment 

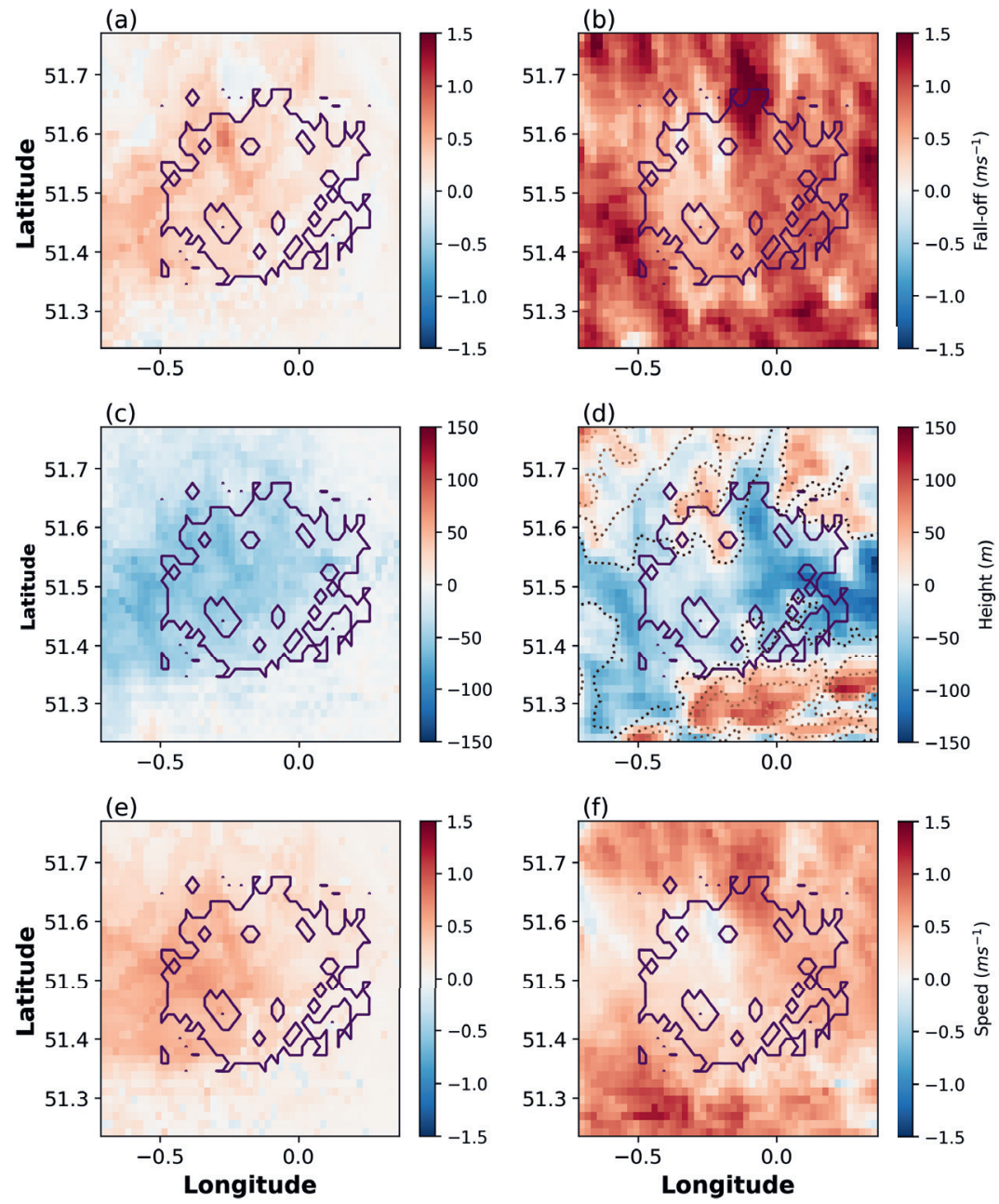

Figure 4.6.2: Spatial distribution of the difference in modelled WRF time-averaged LLJ characteristics (fall-off, height and peak speed) within and around London between the reference and cropland (cropland - reference) (a,c,e) and the reference and notopo (notopo reference) (b,d,f) WRF experiments during the first night (2100 UTC 14/05/2019 to 0600 UTC 15/05/2019). The boundaries of London's urban area are defined (black line) using the land-use index from WRF.

(Figs. 4.6.2b,f and A.4.4b,f) indicates that the wind speed in the upper part of the residual layer also decreases. This is an effect of the lower geostrophic wind (by $\approx 0.5 m s^{-1}$ ) in the notopo experiment.

From our previous analysis it is clear that topography has a substantial effect on the LLJ 
characteristics and their spatial distribution. The topographic effect on LLJ characteristics is dominant upwind (east), south and north of London, while over the city (Fig. 4.6.1) and downwind (west) the effects of urban land use and topography are comparable in magnitude.

\subsection{LLJ effects on the TKE in the nocturnal UBL}

Understanding the TKE evolution during LLJ events can provide evidence whether the LLJ is actually increasing the nocturnal UBL height and whether there is evidence of an "upside-down" boundary-layer structure. Thus, we investigate the changes in the nocturnal production of TKE between 50-300 m over London and the surrounding rural areas utilizing the reference and two idealized (cropland, notopo) WRF experiments. We avoid extending the averaging to include the lowest two model levels (25 and 50m) as the urban roughness effect will be dominant there. Note that the shear and buoyancy production of TKE in the MYNN scheme are proportional to the mixing length, which also depends on the total amount of TKE at each model level. We also investigate the changes in the total TKE and mixing length over London during both nights.

Shear is the dominant source of TKE production during both nights and it outweighs the destruction of TKE through buoyancy maintaining the turbulent mixing within the nocturnal UBL (Figs. 4.7.1a,b,c,d,g,h). The TKE produced by the shear under the LLJ nose helps to maintain a mixing length of more than $10 \mathrm{~m}$ within the nocturnal UBL and reduces the decay rate of TKE after the collapse of the daytime UBL (around 2000 UTC) during both nights. This finding support the hypothesis that the enhanced mixing over London, and consequently the increased UBL depth, increases the height of the LLJ and slightly decrease its speed, which is consistent with the finding of Klein et al. (2016) that showed an increase in LLJ height and decrease in LLJ speed for higher eddy diffusivity in their study over Oklahoma city.

The shear produced TKE in the 50-300m layer maintains a constant value between 1900 and 2300 UTC 14 May 2019 (Figs. 4.7.1a,b). This indicates that the shear production of TKE counteracts the decrease of the mixing length after the collapse of the daytime UBL (Figs. 4.7.1a,b,g,h). An increase in shear-produced TKE during the second night (1900 UTC 15 May 2019 to 0100 UTC 16 May 2019) is reported, leading to an increase in the mixing length between 2200 UTC 15 May 2019 and 0000 UTC 16 May 2019. The vertical mixing induced by the LLJs decreases after midnight and it is not sufficient to maintain the UBL depth leading to a lower mixing length.

The dependency between mixing length and destruction/production of TKE is also evident from the response of the buoyancy production/destruction term. In the beginning of the night (at 2100 UTC) the kinematic heat flux becomes negative at $175 \mathrm{~m}$ a.g.l. (Fig. A.4.3) leading to buoyant suppression and destruction of TKE in the 50-300m layer. Af- 

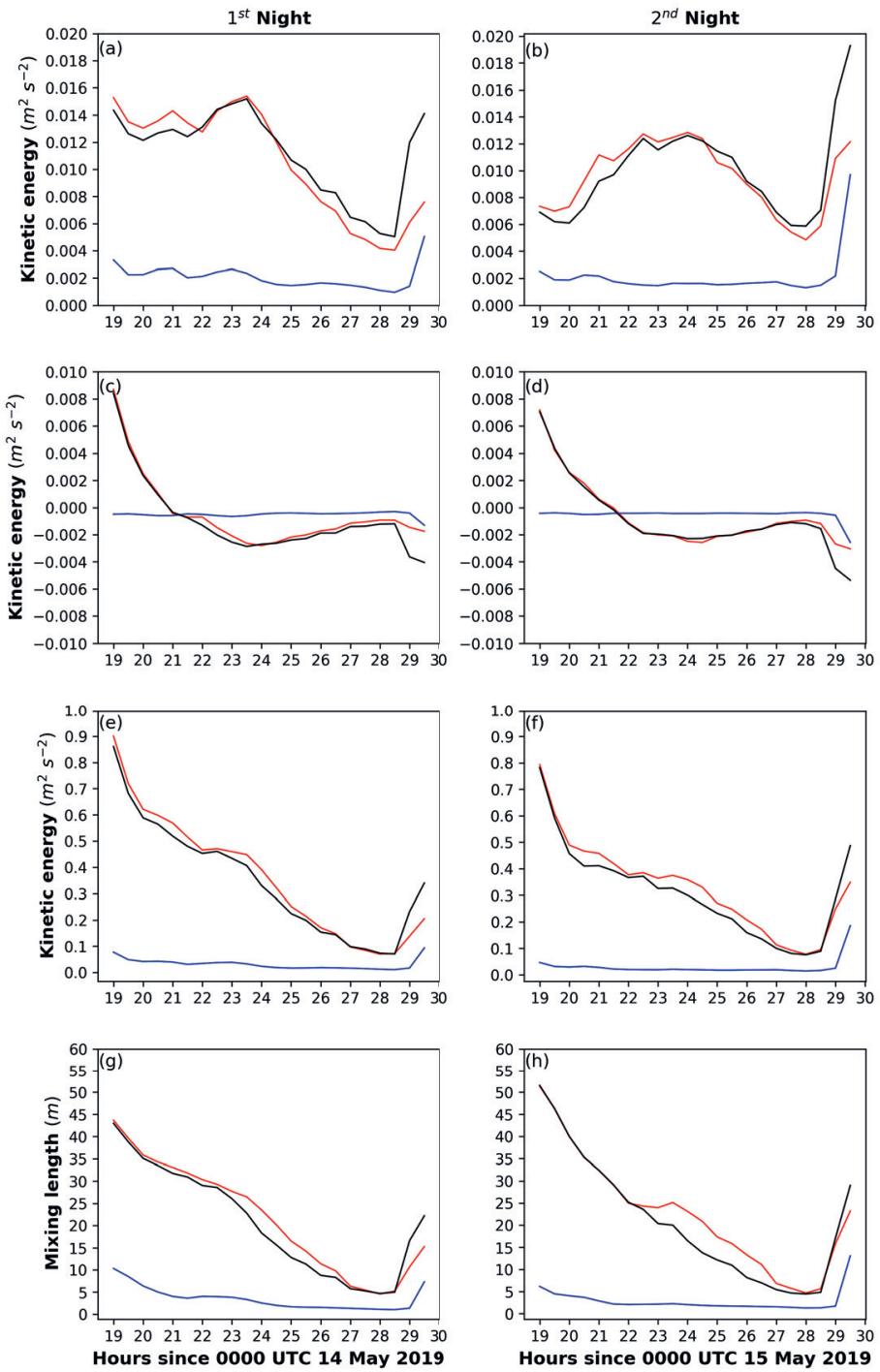

Hours since 0000 UTC 15 May 2019

- Reference - Cropland - Notopo

Figure 4.7.1: Average shear-produced (a,b) and buoyancy-produced (c,d) TKE, average TKE $(\mathrm{e}, \mathrm{f})$ and mixing length $(\mathrm{g}, \mathrm{h})$ from WRF between $50-300 \mathrm{~m}$ a.g.l over the centre of London (LCZs 2,5) during the first (a,c) (1900 UTC 14/05/2019 to 0500 UTC 15/05/2019) and second night (b,d) (1900 UTC 15/05/2019 to 0500 UTC 16/05/2019) of the case study period for the reference (red), cropland (blue) and notopo (black) experiments. 

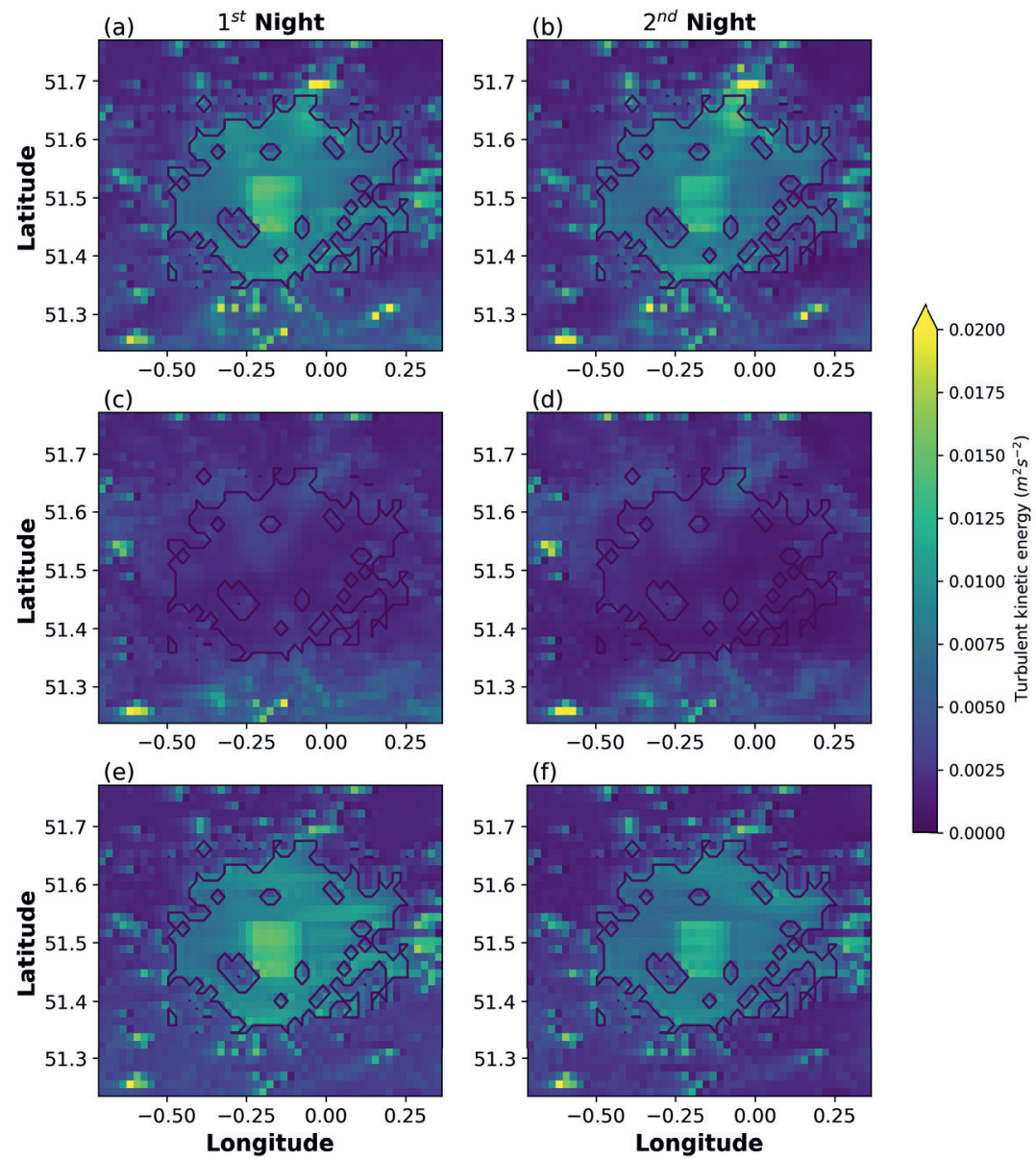

Figure 4.7.2: Average shear-produced TKE in WRF between 50-300m a.g.l. over London for the reference $(\mathrm{a}, \mathrm{b})$, cropland $(\mathrm{c}, \mathrm{d})$ and notopo $(\mathrm{e}, \mathrm{f})$ experiments. The results are an average of the half-hourly values during the first (a,c,e) (1900 UTC 14/05/2019 to 0500 UTC 15/05/2019) and second (b,d,f) (1900 UTC 15/05/2019 to 0500 UTC 16/05/2019) nights of the case study period. The boundaries of London's urban area are defined (black line) using the land-use index from WRF.

ter 0000 UTC both the kinematic heat flux increases and the mixing length decreases, leading to lower buoyant suppression (Figs 4.7.1c,d,g,h, A.4.3). The effects of the mixing length on the production/destruction of TKE are also evident after sunrise (0500 UTC), when the solar radiation heats the surface and increases the UBL mixing length.

The effects of the LLJ-induced shear are more prevalent over the urban area of London, due to the increased TKE generated by the enhanced roughness of the urban surface 
(Figs. 4.7.2a,b) and the higher surface sensible heat flux, which leads to increased mixing lengths during the night. This is also evident in the cropland experiment, where the shearproduced TKE is much lower. We find some evidence that the shear-produced TKE also increases in the cropland experiment during the night (Fig. 4.7.1a,b). However, the lower mixing length in the cropland experiment seems to be the main driver of the differences in TKE production/destruction. The topography is the primary source of shear-produced TKE outside London(Figs. 4.7.2e,f), but has only a small effect on the shear-produced TKE production over central London (Figs.4.7.1c,d,e,f) because terrain elevation is very low.

\subsection{Discussion}

Here we discuss the differences in the initialization time (0000 UTC 14 May 2019 for WRF vs 0900 UTC 14 May 2019 for UKV) and model setup between the WRF and the UKV, that could affect the model performance. An initialization time of 0900 UTC is not possible for WRF as the ECMWF operational analysis data are available every 6 hours (0000 UTC, 0600 UTC etc.). A comparison between the reference WRF run (initialized at 0000 UTC) and a run initialized at 0600 UTC showed a slightly worse performance at capturing the LLJ characteristics over London. Thus we decided to use 0000 UTC run as the reference. The UKV also utilizes a different model suite and data assimilation to produced optimal initial conditions at the start of the model run. Although the additional data assimilation in the UKV has the potential to improve the performance, we do not consider it a substantial difference between the two models as WRF also uses the operational ECWMF analysis fields for initialization, which are adjusted based on observations.

Differences between rural (Chilbolton) and urban (London) LLJ characteristics are investigated for two nights with easterly wind over south-east England. Each night exhibits somewhat different synoptic condition leading to a more baroclinically driven LLJ during the first night, while during the second night an inertial oscillation seems to drive the LLJ formation. For the London we find a strong dependence of the LLJ characteristics on the surrounding topography during both nights. This is in contrast to the findings of Banta et al. (2003) for CASES99, but elevation differences over the CASES99 site were typically smaller than 50m, while terrain heights around London can exceeds 300m (Fig 4.2.1c). We also found indications of potential flow channelling east of London (upwind) that could be responsible for an elevated LLJ height at that area. The topographic orientation with respect to the background wind flow might be important for the spatial distribution of LLJ characteristics. Thus, more case studies need to be conducted, preferably with different background wind directions to test whether the urban and topographic effects on the LLJs are direction dependent. Moreover, the coastal effects on the LLJ over London should also be further investigated 
In section 4.5 we reported an anti-correlation between spatial changes in LLJ height and LLJ speed within and around London. One possible explanation is that the LLJ height increases as the jet flows over the urban surface where the UBL is deeper and there is more vertical mixing. When this transition from lower to higher UBL areas occurs, the turbulent drag experienced by the LLJ increases and thus the LLJ speed decreases. During the transition from higher UBL areas to lower UBL areas the turbulent drag decreases and the jet accelerates back to its nominal "background" wind speed. This LLJ response has been observed over Oklahoma city Wang et al. (2007), would be consistent with the relation between jet height, jet speed and boundary-layer eddy diffusivity (i.e. vertical mixing) reported in Klein et al. (2016) and the relation between atmospheric stability and speed, described in Banta et al. (2006). This hypothesis is also supported by the decrease in LLJ height and increase in LLJ speed over London when the urban areas is replaced by cropland (section 4.6). However, it is likely that in the complex topography around London also contributes to the reported anti-correlation. One consequence of the change in LLJ height and speed during the transition from lower to deeper nocturnal UBLs is that under favourable stability conditions, this "wave-like" motion of the LLJ nose may allow for generation of gravity waves over urban areas.

The complex topography around London and its proximity to the coast makes it very difficult to apply LLJ scaling approaches derived from LLJ studies over homogeneous and flat terrain (Banta et al., 2006; Klein et al., 2016), or to compare the results directly with more conceptual Van de Wiel et al. (2010) and analytical LLJ models Shapiro et al. (2016). This inhibits the comparison of the current results with those of the Joint Urban 2003 campaign over Oklahoma city (Wang et al., 2007; Lundquist \& Mirocha, 2008). However, presence of the complex topography offers the possibility to study potential flow channelling effects on the LLJ and also the effects that interactions between the topography and the urban area might have on the LLJ. Hence we recommend more studies of LLJs over complex terrain to be conducted to test these interaction.

The changes in the shear-produced TKE over the urban area is derived solely through the WRF model simulations. Since there are no available observations, it is not possible to confirm the effects of shear on the vertical mixing in the nocturnal UBL. As the shear-produced TKE is dependent on the mixing length calculated by the boundary-layer scheme, it is also likely that another process (i.e. advection) also contributes to the slower decrease in the UBL's mixing length after sunset. Thus the effect of LLJ advection of the shear-produced TKE in the UBL needs to be tested with an eddy resolving model (i.e. LES model) as well.

During the Joint Urban 2003 campaign over Oklahoma Lundquist \& Mirocha (2008) reported LLJ cases that a exhibit and "upside-down" turbulent structure in the nocturnal UBL with turbulent motions driven by the shear generated under the LLJ nose. Similar evidence has been presented by Banta et al. (2006) for the CASES99 campaign. However, 
in our study we did not find any evidence of similar turbulent structure in the nocturnal UBL over London using the TKE derived from the WRF model. The generally lower LLJ speed $\left(10 \mathrm{~m} \mathrm{~s}^{-1}\right)$ during our case study period could be the reason for the lack of "upside-down" UBL structure, as Banta et al. (2006) reported that this effect usually occurred when the wind speed was larger than $15 \mathrm{~m} \mathrm{~s}^{-1}$. However, it is also likely that it arises from an inability of the model to accurately capture the vertical distribution of TKE in the UBL, which might improve with a finer vertical resolution.

\subsection{Conclusions}

In this study we investigate the interactions between low-level jets and the nocturnal urban boundary layer during a 60-hour case study period over London. Two Doppler Lidars, in combination with two different NWPs (WRF and UKV) are used to identify differences in the LLJ occurrence frequency and characteristics (fall-off, height, speed) between an urban (London) and an rural (Chilbolton) site. Two idealized WRF experiments are used to isolate non-urban (i.e. topographic) effects on the LLJs. Moreover, the impact of LLJ on shear-produced TKE in the nocturnal UBL is analyzed using the WRF reference setup and two sensitivity experiments.

Strong LLJs were detected over both sites during both nights of the case study period. Both models were able to capture the timing and characteristics of the LLJs, but had the tendency to dissipate the LLJ earlier than observed, causing a negative bias in the LLJ speed. Moreover, both models had difficulties at capturing the wind speed in the upper part of the residual layer, causing a bias in the modelled LLJ fall-off.

LLJs over London were located 60-90 m higher than over Chilbolton. This difference in the LLJ height was observed using the Doppler Lidar and was also simulated by both models. The difference in LLJ speed between the two sites is significant during the first night $\left(2.0 \mathrm{~m} \mathrm{~s}^{-1}\right)$, but not during the second night $\left(<0.3 \mathrm{~m} \mathrm{~s}^{-1}\right)$. This indicates that the reported differences in the LLJ characteristics between the two sites are not only affect by the increased turbulent mixing over London, but also by the differences in topography and baroclinicity between the two sites. Through a series of sensitivity tests we identified that : a) the topography around London produces larger magnitude effects on the LLJ than the urban area of London, b) but the urban area does cause significant and spatially relevant effects (i.e. downwind plume effects).

An anti-correlation between the spatial distribution of the LLJ height and speed is found over London, associated with spatial differences in the UBL depth. We find that an increase in UBL depth increases the LLJ height and decreases the LLJ speed. This effects is probably caused by enhanced turbulent drag in the sub-jet layer. The decrease in LLJ speed also decreases the LLJ fall-off.

Finally, we find that shear-produced TKE, between the LLJ and the urban surface, either 
persists ( $1^{\text {st }}$ night) or increases over time ( $2^{\text {nd }}$ night) between 1900 UTC and 0000 UTC. Although no direct increase in the total TKE or mixing length is reported, we do find a slower decrease rate for these quantities after sunset. This indicates that the LLJ together with nocturnal heat release from the urban surface can delay the collapse of the UBL and maintain part of the turbulent mixing.

\section{Acknowledgements}

This research has been funded by the NWO grant no. 864.14.007. The authors would like to thank the ECMWF for providing operational analysis data used for model initialization and forcing. We would also like to thank the UK Met Office and Humphrey Lean for providing operational data from the UKV model used in the research. 



\section{Chapter 5}

On the role of turbulence and momentum advection in the formation of nocturnal low-level jets over urban areas 


\section{Abstract}

Low-level jets (LLJs) are wind maxima that usually occur over the nocturnal boundary layer. The interactions between urban areas and LLJs are important, as the change in wind speed caused by the LLJ can affect street-level heat ventilation and the transport of pollutants away from urban areas. The increased turbulent mixing in the nocturnal urban boundary layer (UBL) can affect the LLJ formation. In this study, we investigate the effect of turbulent mixing in the nocturnal UBL and the role of momentum advection on the LLJ formation over cities. We use two models, the single-column version of the Weather Research and Forecasting model (1D-WRF) and the state-of-the-art PALM Large Eddy Simulation model with explicit representation of the urban areas, in an idealized 4-hour case study. Using the 1D-WRF model we find that increasing urban fraction leads to a delay in the onset of the LLJ (up to 1.5 hours), while the LLJ is elevated (up to $100 \mathrm{~m}$ ) and its speed is decreased (up to $2 \mathrm{~ms}^{-1}$ ). These results are supported by those of the grassland and "infinite" city PALM LES experiments. Moreover, we conducted a PALM experiment with a concentric city surrounded by grassland. We find that the LLJ is first formed over the rural surroundings, due to an earlier decoupling compared to the urban area. The LLJ is then advected over the urban area. The contribution of momentum advection to the LLJ formation depends on the city diameter, with a smaller city $(4 \mathrm{~km}$ diameter) having little-to-no impact. The LLJ formation in rural areas downwind of the urban area is affected by the advection of urban-generated TKE that delays the onset of the LLJ. 


\subsection{Introduction}

Low-level jets (LLJs) are maxima in the wind profile that usually occur over the nocturnal boundary layer. Over the years, many studies have investigated the mechanisms behind the formation of low-level jets. The two dominant LLJ generation mechanisms are inertial oscillations (IO) due to collapse of turbulent mixing (Blackadar, 1957; Shapiro \& Fedorovich, 2008; Van de Wiel et al., 2010; Shapiro et al., 2016; Parish, 2017) and baroclinicity either due to sloping terrain or synoptic forcing (Holton, 1967; Kotroni \& Lagouvardos, 1993; Hu et al., 2013b; Shapiro et al., 2016). These two mechanisms are not isolated and can jointly contribute to the formation of the nocturnal LLJs as seen in Shapiro et al. (2016).

LLJs are interesting phenomena not only due to the their complex formation mechanisms, but also due to their influence on the nocturnal boundary-layer (NBL) dynamics. The wind shear generated by the rapid increase of wind speed with height, can increase the vertical mixing and turbulent kinetic energy (TKE) within the NBL (Banta et al., 2003, 2006; Lundquist \& Mirocha, 2008; Pichugina \& Banta, 2010; Banakh \& Smalikho, 2018), thus also increasing the NBL depth. The enhanced vertical mixing induced by the LLJ has implications for air quality (Banta et al., 2004; Bao et al., 2008; Hu et al., 2013b; Klein et al., 2014; Miao et al., 2019) and urban heat islands (Hu et al., 2013b), while the general increase in wind speed and wind shear is also important for wind energy (Emeis, 2014; Kalverla et al., 2017).

The interactions, between LLJs and urban areas is particularly interesting, since the NBL exhibits distinct differences from its rural counterpart. Over rural areas the TKE collapses fast during the evening transition leading to an onset of stable conditions near the surface, which are favourable for the formation of LLJs (Baas et al., 2009). On the other hand, the urban boundary layer maintains part of vertical mixing after sunset, which leads to a delay in the collapse of the TKE (Pal et al., 2012; Barlow et al., 2015). Consequently, several studies have found differences in the occurrence frequency, height and speed of the LLJs between urban and rural areas (Wang et al., 2007; Lundquist \& Mirocha, 2008; Lemonsu et al., 2009; Kallistratova \& Kouznetsov, 2012; Barlow et al., 2015; Klein et al., 2016).

The results collected during the Joint Urban 2003 (JU2003) over Oklahoma have been particularly interesting in elucidating the interaction between LLJ and urban areas. Wang et al. (2007) found that LLJ can be elevated over the central business district of Oklahoma, due to enhanced vertical mixing in the nocturnal UBL, while downwind of urban area the LLJ speed was decreased (Lemonsu et al., 2009). Lundquist \& Mirocha (2008) and Barlow et al. (2015) found that the presence of LLJs can increase the TKE within the boundary layer, leading to a formation of an "upside-down" boundary layer, where turbulent mixing is driven by wind shear generated under the LLJ nose, rather than the wind shear near 
the surface (i.e. due to surface roughness).

The aforementioned studies highlight the influence of turbulent mixing on the height and speed of the LLJ. Considering that LLJs are generated as a result of the collapse of turbulent mixing during the evening transition, it is expected that enhanced vertical mixing over urban areas might affect the LLJ onset. Indeed, ground-based remote sensing observations of LLJs over London confirmed the delayed collapse of the daytime UBL, due to the presence of an "urban convective island". This may delay the onset of the inertial oscillation and consequently the LLJ formation over London (Barlow et al., 2015). Therefore, Barlow et al. (2015) hypothesised that due to the time difference between the collapse of the rural and urban daytime boundary layers, LLJs are formed first over surrounding rural areas and are then advected over London. If true, this finding has implications for generation of LLJs in rural areas downwind of the city, as urban plumes can transport air with a relatively high TKE over the rural areas. Understanding how interactions between urban and rural areas affect the LLJ formation is an essential step to better understand LLJ dynamics and to be able to accurately represent LLJs in numerical weather prediction models (NWPs).

More specifically, the role of momentum advection on the LLJ formation over cities and its relation with the turbulent mixing in the UBL is still uncertain. Relatively high turbulent mixing over urban areas can reduce wind speeds causing horizontal wind speed gradient between the city and the countryside, which can initiate momentum advection. Yet, a series of questions still remain unanswered. Does the city size affect the formation and characteristics of the LLJ? What is the relation between urban fraction and turbulent mixing? Does the turbulent mixing in the UBL affect the LLJ formation downwind of the urban area? These questions are difficult to answer using observations as they are sparse and thus insufficient to obtain a full spatial insight in the phenomenon. However, these questions can be unravelled by a modelling approach, where turbulent transport below the LLJ is resolved and the spatial heterogeneity of the urban area is captured.

In this chapter the evolution of a low-level jet over an idealised city is studied, to unravel how the LLJ formation is affected by the turbulent mixing intensity during the evening transition. Moreover, we quantify the contribution of advection and geostrophic forcing on the evolution of a nocturnal LLJ both over the city and the countryside. For this purpose an idealised case study based on a real situation during the 15 May 2019 over London is used. This chapter is organised as follows. Section 5.2 describes the case study, the modelling approach and other methodological details. The effects on vertical mixing on the LLJ formation are investigated in section 5.3, while in section 5.4 we describe the role of momentum advection on the LLJ formation over the city. Finally, discussion (section 5.5) and conclusion (section 5.6) are presented. 


\subsection{Methodology}

\subsubsection{Modelling setup}

Two different modelling setups are used, to investigate the effect of vertical mixing and the role of momentum advection on the LLJ formation over urban areas: (a) the singlecolumn (1D) version of the Weather Research and Forecasting (WRF) model (Skamarock et al., 2008) and (b) the state-of-the-art Large Eddy Simulation (LES) model PALM (Maronga et al., 2020).

\section{The single-column Weather Research and Forecasting model}

The 1D-WRF model is based on version 3.8.1 (Skamarock et al., 2008). For the parameterization of the physical processes in urban area we use the single-layer urban canopy model (SLUCM) (Kusaka et al., 2001; Chen et al., 2011a), while the vegetated surface is parameterized with the Noah-LSM scheme (Chen \& Dudhia, 2001). The energy balance components are combined using a tiling approach based on their plan area fraction. Urban surface parameters followed the default settings for high-density residential areas in SLUCM (Chen et al., 2011a; Loridan et al., 2010), but with a mean building height of $20 \mathrm{~m}$.

The surface and boundary layer are parameterized using the revised MM5 (Jimenez et al., 2012) and Mellor-Yamada Nakanishi Niino (MYNN) 2.5 order closure scheme (Mikio \& Hiroshi, 2009). Radiative processes for long-wave and short-wave radiation are obtained using the RRTMG radiation schemes (Iacono et al., 2008), while for microphysics the WSM 3-class scheme (Hong et al., 2004) is used. The model uses 70 vertical levels that extent to $12 \mathrm{~km}$, with 30 levels within the lowest $1.5 \mathrm{~km}$.

\section{PALM model version 6}

The PALM model version 6 (Maronga et al., 2020) revision cycle 4323 is used. PALM uses the Boussinesq approximation for the governing equations (Maronga et al., 2015, 2020). An additional multi-grid pressure solver is used (Maronga et al., 2015). PALM utilises a 1.5-order sub-grid closure scheme by Moeng \& Wyngaard (1988) in the LES mode. The advection is handled by the second-order central difference scheme of Piacsek \& Williams (1970). Cyclic boundary conditions are applied at the edges of the simulated domains. A third-order Runge-Kutta scheme is applied for the time discretization. For more information on the PALM core we refer to Maronga et al. (2015).

The land-surface processes in PALM are parameterized with a scheme (Maronga et al., 2020) similar to that employed by the ECMWF (Balsamo et al., 2009) and DALES (Heus et al., 2010). To estimate fluxes and energy balance at the different facets of building an urban building model is used (Resler et al., 2017). The fluxes are estimated in a similar 
manner as in the LSM model (Maronga et al., 2020). Additionally, a radiative transfer model (RTM), coupled with the radiation code of RRTMG (Clough et al., 2005; Maronga \& Reuder, 2017), is applied to calculated radiative fluxes at the different surface elements (walls, roof, trees etc.) (Resler et al., 2017; Maronga \& Reuder, 2017; Maronga et al., 2020). The RTM model is able to account for shading effect and reflections on vertical and horizontal surfaces (Resler et al., 2017).

\subsubsection{Experimental design}

The 1D-WRF model is used for an initial quantification of the urban fraction effects on the formation and characteristics of the low-level jet, because the PALM model is computationally expensive to perform multiple simulations needed to quantify this effect. Moreover, as PALM has not been used before to study the LLJ formation over urban areas, the 1D-WRF also works as measure of comparison. Note that in this idealised case study, no advection of temperature, moisture or momentum is applied in the 1D-WRF model. Therefore, the evolution of the LLJ only depends on the geostrophic wind forcing and the effect of the urban surface on the turbulent drag within the UBL. The 1D-WRF results are given as 10-minute instantaneous values.
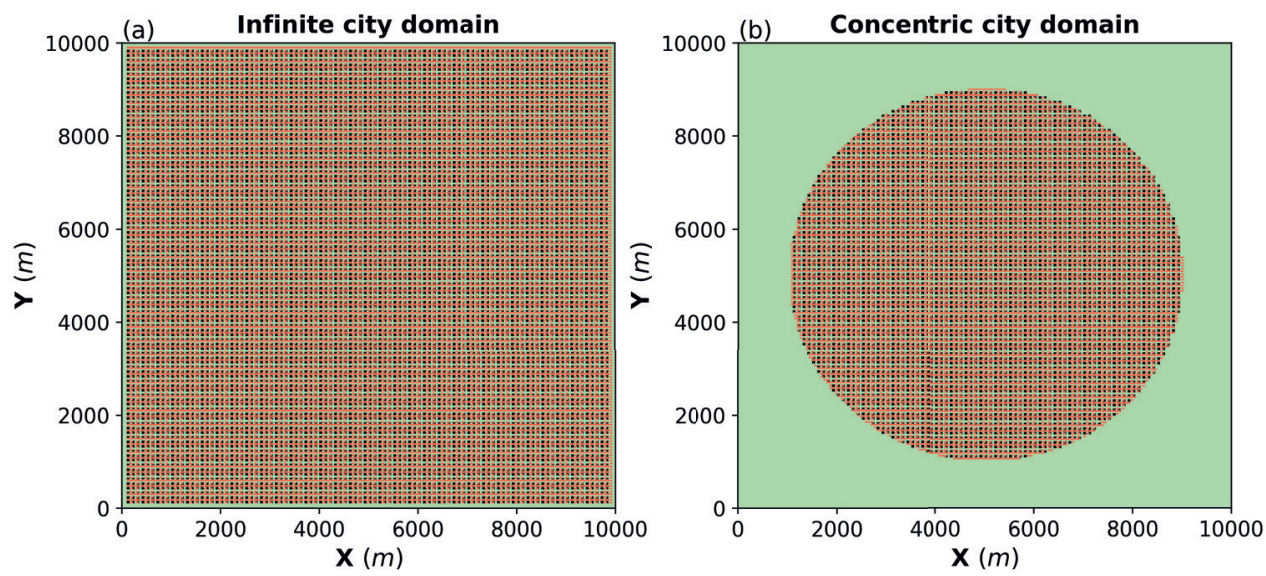

Figure 5.2.1: Illustrations of (a) the infinite city and (b) the concentric city domain for the PALM model. Building gridcells are coloured black, while pavement is red and grassland is green. Note that the concentric city domain extends another $10 \mathrm{~km}$ in each direction for a total of $30 \times 30 \mathrm{~km}$ domain, here we only show the layout of the urban area in the center of the domain

The PALM model is used in a number of different configurations. A $10 \times 10 \mathrm{~km}$ domain is used to simulate a fully rural (grassland) and an urban domain (Figure 5.2.1a). The urban domain consists individual building elements (60m width, height and length) separated by patches $(40 \mathrm{~m})$ of pavement and grassland to create an urban area of approximately 
$70 \%$ urban fraction. These two experiments are compared to the $1 \mathrm{D}-\mathrm{WRF}$ simulation to test the performance of the PALM model and ensure that it captures the LLJ formation over both vegetated and urban areas. Note that the urban and rural domains use cyclic boundary conditions and thus are effectively an "infinite" grassland and an "infinite" city. Although there is advection present within the domains, there is no external largescale advection or subsidence that originates from differences between the city and the countryside (same as in the 1D-WRF model).

To test the role of the momentum advection a mixed (city-rural) PALM run is conducted. A 30x30 km domain is used with a concentric city of $8 \mathrm{~km}$ diameter located in the centre of the domain, while the remain area is covered by grassland (Figure 5.2.1b). Note that within the city the building, pavement and grassland distribution is similar to that in the "infinite" city experiment. This experiment, labelled "concentric" city, is repeated for cities of different diameter $(4,8$ and $12 \mathrm{~km})$ to test the role of city size on the momentum advection. Note that all PALM runs use $20 \mathrm{~m}$ horizontal and vertical resolutions, with a model top at $1620 \mathrm{~m}$. This resolution is not able to fully resolved the eddies in the lowest 2-3 models levels, where most of the turbulence is parameterized. However, above $80 \mathrm{~m}$ and throughout the remaining vertical levels the majority of the turbulence $(>90 \%)$ is resolved. Higher vertical and horizontal resolution would have been computationally too expensive for these model experiments. In addition, Rayleigh dampening is applied above $1250 \mathrm{~m}$ to ensure that the flow at the domain top follows the initially prescribed wind, temperature and moisture. Output data are $10 \mathrm{~m}$ averaged $3 \mathrm{D}$ fields of $\mathrm{u}, \mathrm{v}$ and $\mathrm{w}$ wind components and turbulent intensity. Fluxes, are provided as 2D (height-time) horizontally averaged values every 10 minutes.

\subsubsection{Case study description}

The idealised case study is based on a real meteorological situation between 1700 UTC 15 May 2019 and 0500 UTC 16 May 2019, over London, UK. This is a modified version of second night of the 14-16 May 2019 case study used in Chapter 4. During this period a LLJ forms over London, starting at 2200 UTC 15 May 2019 and lasting until 0500 UTC 16 May 2016. The meteorological forcing for the 1D-WRF and PALM models is derived by a 3D-WRF simulation spanning the entire length of the 14-16 May 2019 case study period, with similar parametrization settings.

For the 1D-WRF, initial profiles of potential temperature, specific humidity and $\mathrm{u}, \mathrm{v}$ wind components are applied up to a height of $15 \mathrm{~km}$ (Figure A.5.1). Similar profiles, including an initial profile for vertical wind, are used up to a height of $1620 \mathrm{~m}$ to initialise the PALM model (Figure A.5.2). These initial profiles have been derived from a WRF-3D simulation at 1700UTC 15 May 2019 and are an spatial average of the relavant quantities in an area of $30 \times 30 \mathrm{~km}$ around the centre of London. Initial profiles of soil temperature and moisture are obtained from the 3D-WRF run and applied to the 1D-WRF and the 
PALM models (Figure A.5.4). Hourly $\mathrm{u}$ and $\mathrm{v}$ geostrophic wind components are applied at each timestep in both models (Figure A.5.3). These values are varying with height as this is essential to form the LLJ during that night, but are constant in time (values at 1700 UTC 15 May 2019 from the 3D-WRF model) to simplify the geostrophic forcing. Note that, PALM model does not allow for simultaneous initialisation of $\mathrm{u}$ and $\mathrm{v}$ wind profile, when the geostrophic forcing is active. Thus, we set the initial $\mathrm{v}$ wind profile to 0 $m s^{-1}$, as the actual observed $\mathrm{v}$ wind component in the boundary layer is close to $0 \mathrm{~ms}^{-1}$ at 1700UTC.

The period of the idealised case study covers only the initial phase of the LLJ formation (1700-2100 UTC) until the LLJ speed reaches a maximum. Since the case study is heavily idealised (no topography, no large-scale advection and modified v wind profile) the LLJ appears earlier than observed and consequently it reached a maximum intensity by 2100 UTC.

Table 5.2.1: Experiments conducted with the 1D-WRF and PALM models. Naming for the 1D-WRF runs use the urban fraction $\left(f_{\text {urb }}\right)$ and its tested values. The focus of each a experiment is either on the effects of turbulent mixing, or momentum advection or both.

\begin{tabular}{ccc} 
Experiment name & Model & Focus \\
\hline$f_{\text {urb }}$ value $(0-1.0$, interval 0.1$)$ & 1D-WRF & Turbulent mixing \\
grassland & PALM $(10 \times 10 \mathrm{~km})$ & Turbulent mixing \\
infinite city & PALM $(10 \times 10 \mathrm{~km})$ & Turbulent mixing \\
concentric city $(4 \mathrm{~km})$ & PALM $(30 \times 30 \mathrm{~km})$ & Turbulent mixing + momentum advection \\
concentric city $(8 \mathrm{~km})$ & PALM $(30 \times 30 \mathrm{~km})$ & Turbulent mixing + momentum advection \\
concentric city $(12 \mathrm{~km})$ & PALM $(30 \times 30 \mathrm{~km})$ & Turbulent mixing + momentum advection
\end{tabular}

\subsubsection{LLJ detection algorithm}

To detect the LLJs and it characteristics (height, wind speed) the Baas et al. (2009) algorithm is applied with a few modifications. The algorithm defines an LLJ when a low-level wind maximum $\left(>4 \mathrm{~m} \mathrm{~s}^{-1}\right)$ that occurs in the lowest $650 \mathrm{~m}$ above ground level. The low-level wind maximum needs to be least $1.5 \mathrm{~m} \mathrm{~s}^{-1}$ (or 15\%) higher than the wind speed in all overlying levels up to $900 \mathrm{~m}$ above ground level. Details of the algorithm are provided in Chapter 4. Note that before the algorithm is applied the model output from the $1 \mathrm{D}-\mathrm{WRF}$ model is linearly interpolated to match the vertical resolution of the PALM LES model (20m). 


\subsection{Impact of turbulent mixing on the LLJ charac- teristics}

As discussed before, the intensity of turbulent mixing impacts the LLJ height and speed (Wang, 2009; Lemonsu et al., 2006; Klein et al., 2014). Using the 1D-WRF model we investigate the effects of an increasing fraction on the turbulent mixing, the LLJ onset time, height and speed. The results of the $1 \mathrm{D}-\mathrm{WRF}$ are compared to three PALM experiments, the grassland, the infinite city and the concentric city experiments. Note that in the grassland and infinite city experiments we use domain averaged profiles, while in the mixed urban-rural run we average $\mathrm{u}, \mathrm{v}$ wind components and turbulent intensity in a $4 \mathrm{x} 4 \mathrm{~km}$ area over the centre of the concentric city. The LLJ speed, height and initial formation time are identified by applying the aforementioned LLJ detection algorithm.

The LLJ onset time is delayed (up to 1.3 hours) when the urban fraction increases in the in 1D-WRF experiments (Figure 5.3.1a,b). This delay is caused by slower collapse of the daytime UBL when urban fractions increases. A similar difference of 1.5 hours is found between the grassland and infinite city PALM experiments (Figure 5.3.1b), which suggests that enhanced turbulent mixing, due to increasing $f_{\text {urb }}$ delays the formation of the LLJ. The difference in the LLJ formations times over grassland and city support the hypothesis that LLJ are created earlier over rural areas and thus can then be advected over urban areas as proposed by Barlow et al. (2015).

The increase in turbulent mixing due to increasing urban fraction does not only affect the onset time of the LLJ, but also the LLJ height and speed (Figures 5.3.2a,b,c,e). The difference in LLJ height at certain times can vary up to $140 \mathrm{~m}$ depending on the urban fraction (Figure 5.3.1a). The increase in LLJ height varies between 5-14 m per 0.1 increase in $f_{\text {urb }}$, but the increase is non-linear. Changes between $f_{\text {urb }} 0.0$ and $f_{\text {urb }} 0.5$ show a stronger effect on the LLJ height than the changes between $f_{\text {urb }}-0.5$ and $f_{\text {urb }}-1.0$. Similar differences in LLJ height occur between the grassland and infinite city runs with the PALM LES model, with the latter showing an elevated LLJ by $20-40 \mathrm{~m}$ depending on time of day (Figure 5.3.1a). The increase in LLJ height coincides with higher TKE within the urban boundary layer (Figure 5.3.2a,b,c,e). This highlights the dependency of the LLJ height to the turbulent mixing intensity as seen before in Klein et al. (2016).

The increase in turbulent mixing, in experiments with higher $f_{\text {urb }}$, also decreases the LLJ speed and the vertical wind profile between 100-400 m (Figures 5.3.2a,c). Runs with higher $f_{\text {urb }}$ show a smooth LLJ at $\mathrm{t}=8400 \mathrm{~s}$, while runs with lower urban fraction show a more sharp LLJ. This is also the case in the wind speed profile from $t=14400 \mathrm{~s}$. Overall, the LLJ speed decreases (up to $1.9 \mathrm{~ms}^{-1}$ ) with increasing urban fraction (Figure 5.3.1b). The change of speed with urban fraction shows a non-linear behaviour, with changes in mid and high urban fractions $(0.5-1.0)$ decreasing the LLJ speed by -0.12 to $-0.14 \mathrm{~ms}^{-1}$ per urban fraction increment, while in the lower urban fractions the change in LLJ speed 

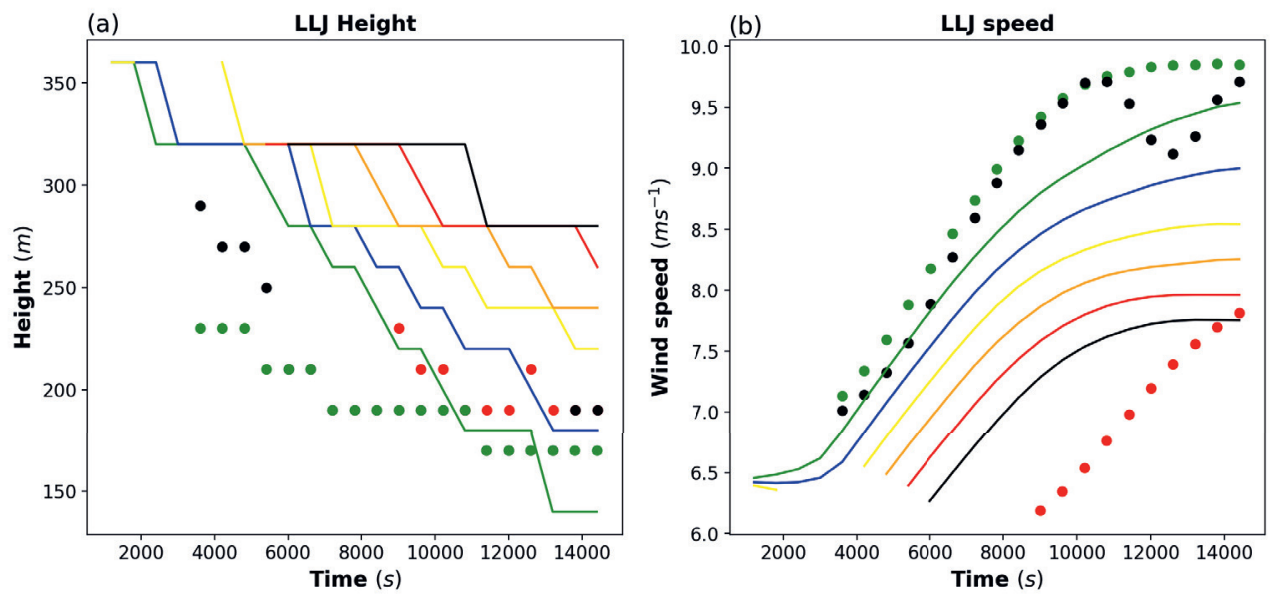

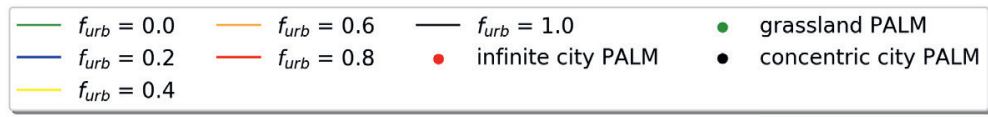

Figure 5.3.1: Temporal evolution (in seconds) of the modelled LLJ height (a) and speed (b) simulated in the 1D-WRF (lines) and PALM (circles) models between 1700 UTC and 2100 UTC 15 May 2019. Experiments with different urban fractions $\left(f_{\text {urb }}\right)$ are depicted with different colours (see legend) as are the PALM grassland (green), infinite city (red) and concentric city (black) runs.

is -0.18 to $-0.20 \mathrm{~ms}^{-1}$.

The 1D-WRF $f_{\text {urb }} 0.0$ and PALM grassland experiments simulate a very similar evolution of the LLJ speed with approximately $0.5 \mathrm{~ms}^{-1}$ difference. The infinite city PALM experiment shows a substantially smaller LLJ speed $\left(-1.0 \mathrm{~ms}^{-1}\right)$ until $\left.\mathrm{t}=14000 \mathrm{~s}\right)$ compared to the $f_{\text {urb }} 0.7$ experiment. The lower LLJ speed, in the infinite city experiment is due it's larger TKE compared to the higher $f_{\text {urb }}$ experiments in 1D-WRF during the initial stages of the LLJ formation (Figure 5.3.2a,c).

The difference in LLJ height between the 1D-WRF and the PALM experiments is substantial. The 1D-WRF experiments shows a sharp decline in LLJ height over time (Figure 5.3.1a) due to a large decrease in boundary-layer height as seen also by the change in the height $(\approx 70 \mathrm{~m})$ of the minimum in the TKE profile (Figures 5.3.2b,d). On the contrary, in the PALM LES experiments the change of LLJ height over time is more modest (less than $100 \mathrm{~m}$ in grassland and infinite city experiments). The concentric city experiment shows the largest decrease in LLJ height during the night. The reason for this deviation is explained in section 5.4.

Differences between the 1D-WRF and PALM TKE profiles are also evident especially 

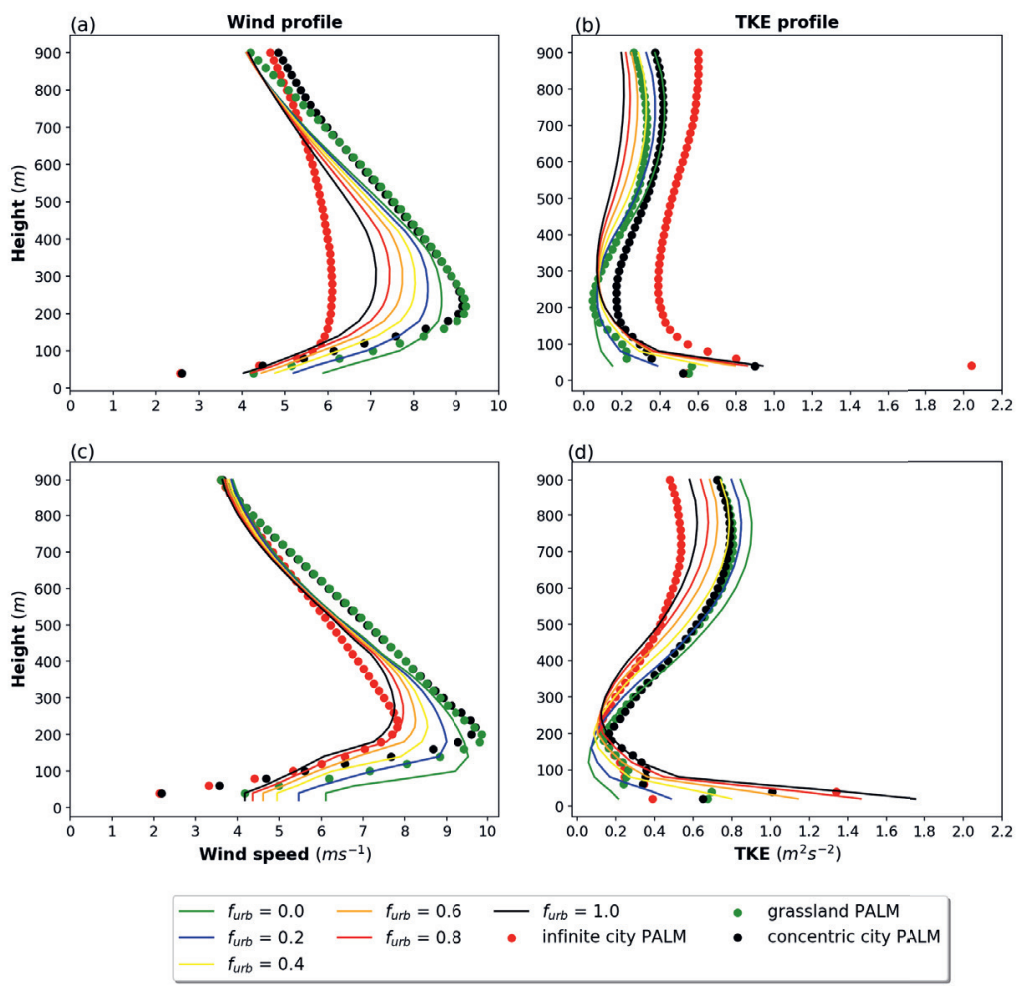

Figure 5.3.2: Vertical profiles over $(\mathrm{a}, \mathrm{c})$ the modelled wind speed $\left(\mathrm{ms}^{-1}\right)$ and $(\mathrm{b}, \mathrm{d})$ total TKE (parameterized + resolved) $\left(\mathrm{m}^{2} \mathrm{~s}^{-2}\right)$ from the 1D-WRF (lines) and PALM (circles) models. The profiles are taken at $\mathrm{t}=8400 \mathrm{~s}(\mathrm{a}, \mathrm{b})$ and $\mathrm{t}=14400 \mathrm{~s}(\mathrm{c}, \mathrm{d})$ after the initialisation of the model runs at 1700 UTC 15 May 2019. Experiments with different urban fractions $\left(f_{\text {urb }}\right)$ are depicted with different colours (see legend) as are the PALM grassland (green), infinite city (red) and concentric city (black) runs.

closer to the surface (bellow 100m) (Figure 5.3.2b,d). The PALM model runs show a much stronger TKE near the surface, especially in the "infinite" city run at $t=8400$ s, due to larger vertical kinematic heat and momentum fluxes near the surface, which is an effect of the urban surface (Figure A.5.5). The impact of the kinematic heat flux on the near surface TKE is more prominent in the early stages of the LLJ formation, while at a later phase both PALM and the 1D-WRF show a strong contribution of the momentum flux on the total TKE budget. In the residual layer, TKE is primarily generated by the vertical momentum fluxes due to the strong wind shear.

Overall, we find that increasing urban fraction results in more TKE and thus a deeper boundary layer, while the LLJ speeds decreases and and the LLJ height increases. These conclusions are consistent with the results of both the 1D-WRF experiments and the 
PALM experiments when rural-to-urban advection is excluded (grassland and infinite city experiments). However, the LLJ evolution and characteristics in the concentric city PALM experiment resemble much more those in the grassland experiment than those of the infinite city experiment. Consequently, it appears that the LLJ over the concentric city is dominated by the influence of the rural surroundings.

\subsection{Turbulent intensity and momentum advection ef- fect in the concentric city experiment}

in this section, the effect of turbulent intensity and the role of momentum advection on LLJ formation over the concentric city is investigated. Note that here turbulent intensity is defined as the absolute value of the rotation vector (units of $1 s^{-1}$ ) and should not be confused with the TKE, although both are a measure of the turbulent mixing. We use the turbulent intensity instead of TKE, because the PALM model does not output 3D fields of the resolved TKE (only the parameterized TKE). In this section we also investigate the effects of the city size on the turbulent intensity and momentum advection at the height of the LLJ (150-230m). This is done by comparing 3 different experiment with concentric cities of different diameters $(4,8$ and $12 \mathrm{~km})$.

(a)

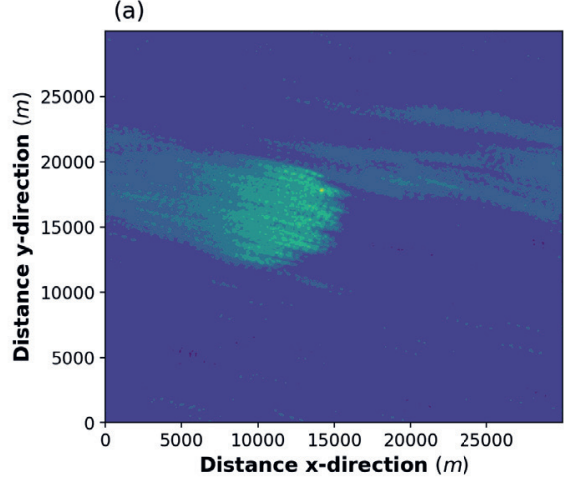

(b)

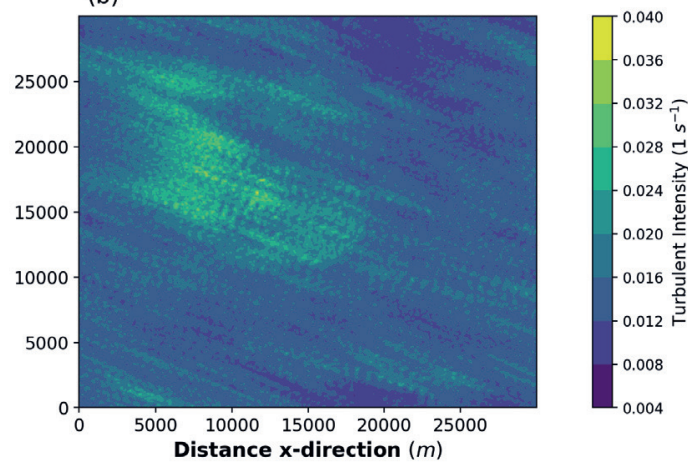

Figure 5.4.1: Spatial distribution of the $160 \mathrm{~m}$ turbulent intensity $\left(1 \mathrm{~s}^{-1}\right)$ at $\mathrm{t}=6000 \mathrm{~s}$ (a) and $\mathrm{t}=9600 \mathrm{~s}(\mathrm{~b})$. Note that the concentric city is located between $11000 \mathrm{~m}$ and $19000 \mathrm{~m}$ in each direction in the PALM domain.

Figure 5.4.2a shows that the turbulent intensity reaches a maximum over the concentric urban area in the domain. This is caused by the generally higher kinematic heat and momentum fluxes over the concentric city compared to the rural surroundings. Yet the enhanced turbulent intensity is not static over the city (Figure 5.4.1a,b and 5.4.2a,b). A pronounced urban plume of high kinetic energy is visible at $\mathrm{t}=6000 \mathrm{~s}$ advecting air with high turbulent intensity downwind of the urban area (Figure 5.4.1a,b). The downwind 


\subsection{Turbulent intensity and momentum advection effect in the concentric}

city experiment

plume extends up to $15 \mathrm{~km}$ downwind of the urban area but it mainly affect the LLJ between the centre of the city $(\mathrm{x}=15000 \mathrm{~m})$ and up to $8 \mathrm{~km}$ downwind $(\mathrm{x}=7000 \mathrm{~m})$. The turbulent plume breaks the inversion downwind of the urban area as seen in Figure $5.4 .2 \mathrm{a}$ at $\mathrm{t}=6000 \mathrm{~s}$ as it increases turbulent intensity up to a height of $250 \mathrm{~m}$. At $\mathrm{t}=$ $9600 \mathrm{~s}$, the plume is still visible but is restricted to lower heights (up to $160 \mathrm{~m}$ ). The difference in turbulent intensity between the plume and the air is lower as the strong LLJ increases the the turbulent intensity also over the rural areas during the night (Figure $5.4 .1 \mathrm{~b})$.
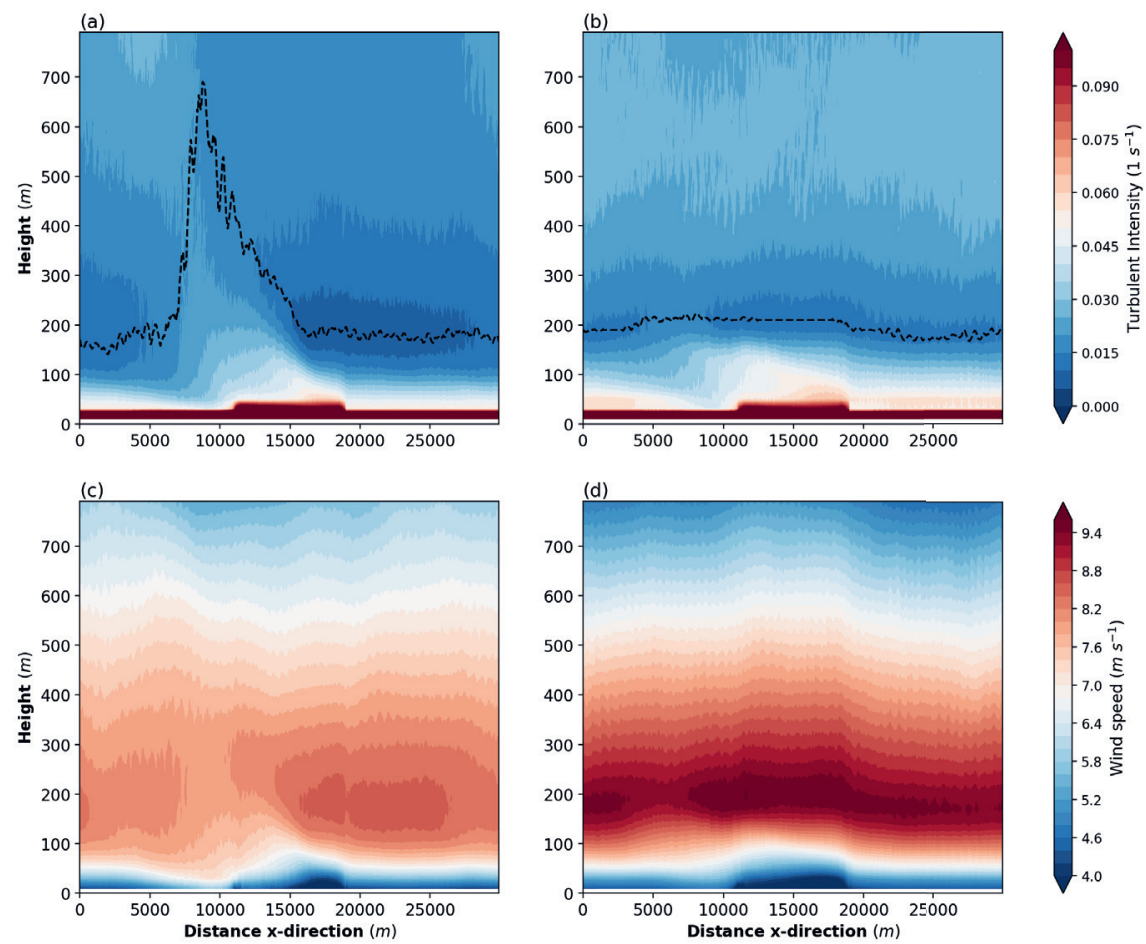

Figure 5.4.2: Vertical height-distance cross-section across the $x$ dimension for the modelled: $(\mathrm{a}, \mathrm{b})$ turbulent intensity $\left(1 \mathrm{~s}^{-1}\right)$ and $(\mathrm{b}, \mathrm{d})$ wind speed $\left(\mathrm{ms}^{-1}\right)$ at $\mathrm{t}=6000 \mathrm{~s}(\mathrm{a}, \mathrm{c})$ and $\mathrm{t}=$ $9600 \mathrm{~s}(\mathrm{~b}, \mathrm{~d})$. Black dotted lines indicate the height of the minimum turbulent intensity. The cross-sections are an average of all cross-sections spanning $4 \mathrm{~km}$ (in the y direction) over the central part of the concentric city. Note that in these cross-sections the city is located between $11000 \mathrm{~m}$ and $19000 \mathrm{~m}$ in the PALM domain. Background wind is from the east.

Note that turbulent intensity is weak above $100 \mathrm{~m}$ in the upwind part of the urban area, which suggests that advection of air with the low turbulent intensity takes places from the rural above $100 \mathrm{~m}$ (Figure 5.4.2a). This is also confirmed by the spatial distribution of the turbulent intensity over the concentric city in the PALM domain (Figure 5.4.1a,b). These 
results indicate that advection processes from rural to urban areas play a crucial role in the vertical profiles of TKE and the vertical structure of the nocturnal urban boundary layer.

The effect of the high turbulent intensity plume is also visible in the vertical profiles of wind speed (Figure 5.4.2c,b). In locations with high turbulent intensity the additional turbulent drag reduced the wind speed and results in a weaker LLJ. This effect is visible between the 100-300 $\mathrm{m}$ above ground level and it extends from the central part of the city up to more than $15000 \mathrm{~m}$ downwind. Moreover, in the upwind part of the city the strong LLJ speed indicates that the LLJ is able to penetrate up to $4 \mathrm{~km}$ within the city before the turbulence starts to erode the LLJ (Figures 5.4.2c). Yet this effect is more pronounced at $\mathrm{t}=6000 \mathrm{~s}$ than an hour later $(\mathrm{t}=9600 \mathrm{~s})$, due to the collapse of the turbulence over the city. At this time, there is no visible break in the inversion and turbulence from the city is confined in the lower $150 \mathrm{~m}$ of the atmosphere. Therefore, a strong LLJ forms across the whole domain. Still, the LLJ is slightly elevated over the urban as seen in Figure 5.4.2d), which agrees with our previous findings in section 5.3 and in the literature (Wang et al., 2007; Klein et al., 2016).

The horizontal wind speed gradient (Figure 5.4.2c) supports the hypothesis of momentum advection from the rural areas contributing to the LLJ formation over the concentric city as proposed by Barlow et al. (2015). Yet the exact contribution needs to be quantified by investigating the contribution of advection and geostrophic forcing on the tendencies of the $\mathrm{u}$ and $\mathrm{v}$ wind components. Here these variables are quantified for three different $4 \mathrm{x} 4$ $\mathrm{km}$ areas in the concentric city PALM domain: a) the centre of the concentric city, b) a downwind rural area above which the plume is located and c) an area in the south that experience minimal impacts of the urban plume.

The $\mathrm{u}$ wind tendency due to ageostrophic wind forcing increases during the night, indicating that until $\mathrm{t}=14400$ s it effectively increases (tendency $<0$ ) the absolute value of the $\mathrm{u}$ wind component (note that $\mathrm{u}<0$ in this case study)(Figure 5.4.3a). The vtendency is positive during the whole night indicating that $\mathrm{v}$ wind component increase due to geostrophic forcing over the whole period (Figure 5.4.3b). This forcing is rather similar for the tendency of the $\mathrm{u}$ wind components in all three areas, but it does fluctuate for the $\mathrm{v}$ wind components due to differences in the $\mathrm{u}$ component of the wind speed over the three different areas (Figure 5.4.3b). Note that geostrophic wind forcing is rather constant with height in the 100-280 m layer as seen if Figures 5.4.4c,d.

The tendency due to $\mathrm{u}, \mathrm{v}$ momentum advection shows a different behaviour over the city, compared to the rural areas south and downwind (Figure 5.4.3a,b). The tendency of $\mathrm{u}$ wind component due to momentum advection peaks at around $t=6000 \mathrm{~s}$ over the urban area, where its value is nearly double that of the tendency due to geostrophic forcing (Figure 5.4.3a). This momentum advection is caused by the horizontal wind gradient over and downwind of the city (Figure 5.4.2c), mainly due to different in the $\mathrm{u}$ wind 

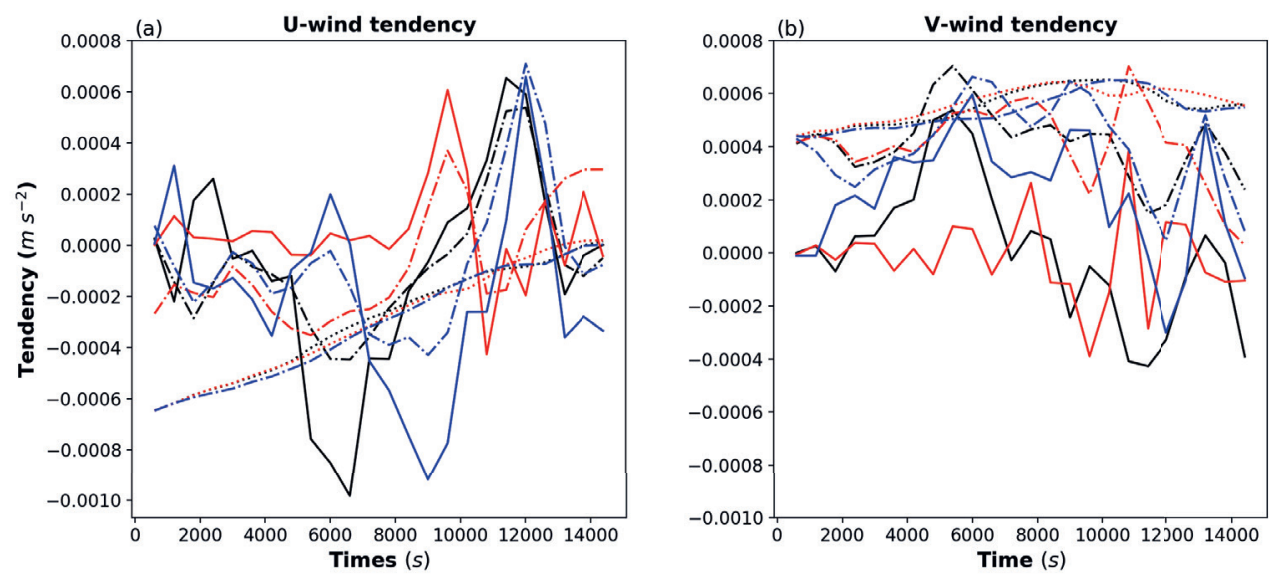

\begin{tabular}{|c|c|c|}
\hline $\begin{array}{ll}\cdots & \text { geo_urban } \\
& \text { advection_urban } \\
\ldots & \text { utend_urban }\end{array}$ & $\begin{array}{ll}\cdots \cdots \cdot & \text { geo_south } \\
- & \text { advection_south } \\
-\cdots & \text { utend_south }\end{array}$ & $\begin{array}{ll}-\cdot- & \text { geo_downwind } \\
- & \text { advection_downwind } \\
-.- & \text { utend_downwind }\end{array}$ \\
\hline
\end{tabular}

Figure 5.4.3: Temporal evolution of the total (a) $\mathrm{u}$ and (v) $\mathrm{v}$ wind tendencies $\left(m s^{-2}\right)$ (dashed lines) and the tendencies due to geostrophic forcing (dotted line) and advection (full line), for the center of the urban area (black) and the rural areas south (red) and downwind (blue) of the city. The tendencies are an average between 150-230 m above ground level.

component. Note that the momentum advection is stronger closer to the surface, but still retains similar strength to the tendency due to geostrophic forcing up to $200 \mathrm{~m}$. Moreover, there is little advection before $t=4000 \mathrm{~s}$. This indicates that the advection occurs after onset of the LLJ at the surrounding grassland (Figure 5.3.1b).

There is very little momentum advection south of the concentric city at $t=6000 \mathrm{~s}$, while downwind of the city there is slightly positive advection indicating the the absolute value of the $\mathrm{u}$ wind component decreases (Figure 5.4.3a). In the area downwind there is evidence of strong momentum advection around $t=9000 \mathrm{~s}$, indicating that the LLJ is able to penetrate the turbulent urban plume downwind of the city.

The $\mathrm{v}$ wind component tendency due to advection shows a similar temporal variability as the $\mathrm{u}$ wind component tendency. It is important to note that for the $\mathrm{v}$-component positive tendency indicates an increase in the $\mathrm{v}$ wind component speed (Figure 5.4.3a). Yet, the contribution of momentum advection on the $\mathrm{v}$ wind component remains smaller than that due geostrophic forcing, even though at certain times they can have similar magnitude.

The total $\mathrm{u}$ and $\mathrm{v}$ wind tendencies over the urban area tend to reach maximum values when there is substantial momentum advection (Figures 5.4.3a,b). Note that the total tendencies, are lower than the sum of tendencies due to geostrophic forcing and advection 

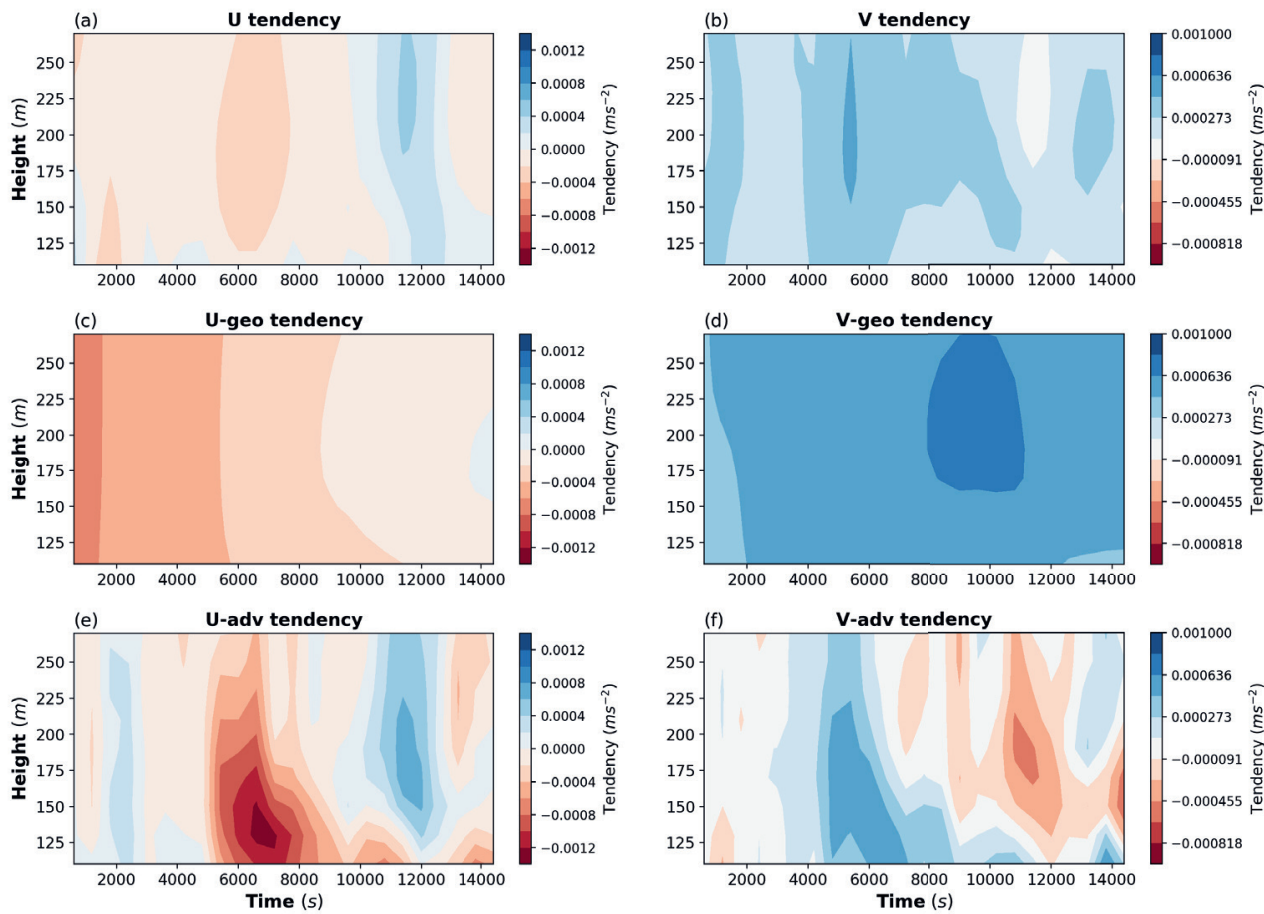

Figure 5.4.4: Time-height cross-section of the total $(\mathrm{a}, \mathrm{c}, \mathrm{d}) \mathrm{u}$ and $(\mathrm{b}, \mathrm{d}, \mathrm{f}) \mathrm{v}$ wind tendencies $\left(m^{-2}\right)(\mathrm{a}, \mathrm{b})$ and the tendencies due to geostrophic forcing $(\mathrm{c}, \mathrm{d})$ and advection of momentum $(\mathrm{e}, \mathrm{f})$, for the centre of the urban area.

as turbulent drag decreases to total acceleration in the $\mathrm{u}$ and $\mathrm{v}$ wind components. The total tendency for both $\mathrm{u}$ and $\mathrm{v}$ wind components peaks between 150-200 $\mathrm{m}$ (Figures $5.4 .4 \mathrm{a}, \mathrm{b})$. Closer to the surface the strong momentum advection is counteracted by the turbulent drag, stopping the formation of a LLJ closer to the ground.

The timing and intensity of the momentum advection strongly depends on the size of the city. In the experiment with the the smaller $4 \mathrm{~km}$ concentric city, there is little turbulence generated by the city and no substantial horizontal gradient in either the $\mathrm{u}$ or $\mathrm{v}$ wind components is visible. Therefore, momentum advection peaks earlier $(\mathrm{t}=$ $4000 \mathrm{~s}$ ) and contributes only slightly towards the total $\mathrm{u}$ and $\mathrm{v}$ wind tendencies (Figure $5.4 .5 \mathrm{a}, \mathrm{b})$. Whereas, in the $12 \mathrm{~km}$ city the turbulence generated is more intense and covers a larger area of the domain. Consequently, the horizontal wind gradient increases leading to stronger and prolonged advection of momentum. Moreover, because of the increased turbulent intensity over the city, the advection of momentum peaks approximately half an hour later compared to the $8 \mathrm{~km}$ run.

Based on these results it is reasonable to assume that the LLJ over the concentric city 


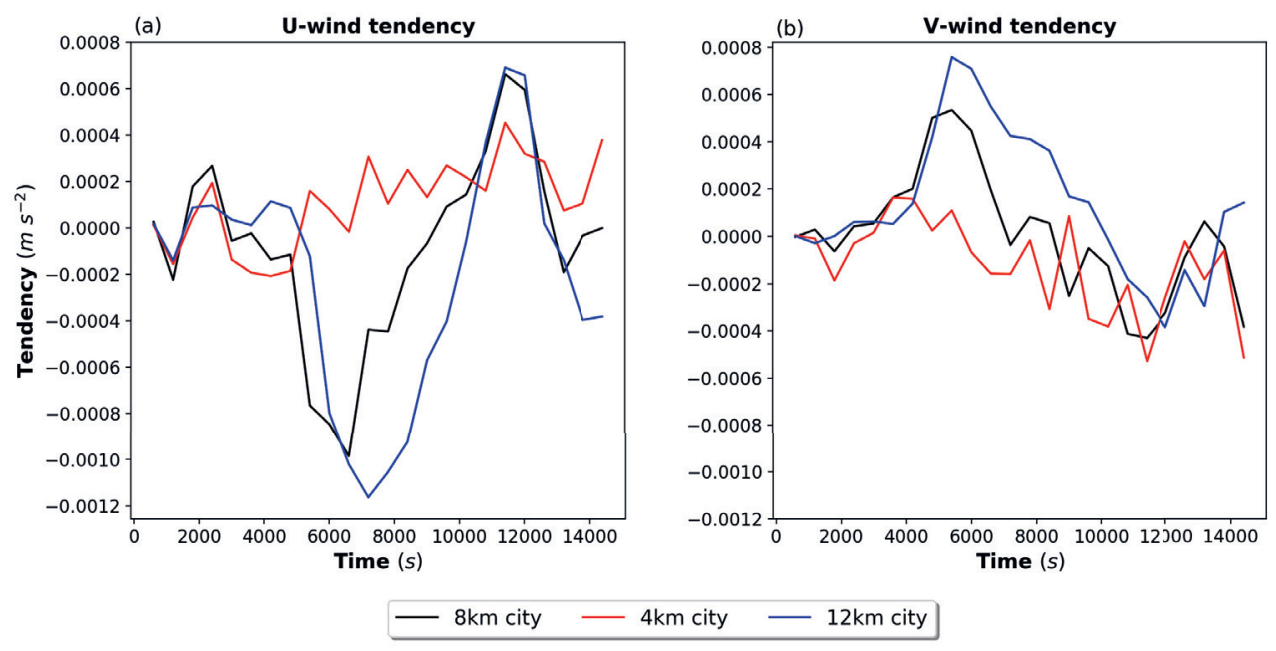

Figure 5.4.5: Temporal evolution of the total (a) $u$ and (b) v wind tendencies $\left(m s^{-2}\right)$ due to advection (full line), for the $4 \mathrm{~km}$ city (red), the $8 \mathrm{~km}$ city (black) and the $12 \mathrm{~km}$ city (blue). The tendencies are an average between 150-230m above ground level.

is formed via a combination of geostrophic forcing and strong horizontal momentum advection. The momentum advection is caused by a horizontal gradient in wind speed, an effect of the stronger turbulent intensity over and downwind of the concentric city. The contribution of momentum advection on the LLJ speed can at times be twice the value of the geostophic forcing, indicating the inertial oscillation is not the only mechanism of LLJ formation over the urban area (at least in this case study). The size of the city has an important role in the advection of LLJ, since smaller cities create less vertical mixing in the UBL resulting in a faster onset of the LLJ over the city.

\subsection{Discussion}

In this study we use a short 5-hour case study period to investigate the impact of turbulent mixing and momentum advection on the formation of LLJ over an idealized city. Our results are based on a specific case study and might differ under different geostrophic wind speeds or due to topographic features surrounding the city and large-scale momentum advection. Thus, it is important to study the relative contribution of momentum advection on the LLJ formation over a wider range of meteorological conditions and for different cities with different topographic characteristics.

The urban areas used in the 1D-WRF and PALM models are only idealised versions of cities, with uniform building height and surface properties, and come in idealised concentric shape. In reality urban areas exhibit a wide range of spatial extents, building 
densities and heights. Moreover, surface properties (i.e. albedo) can vary between cities in different climates and even between different city neighbourhoods due to variations in construction materials. These differences can affect the turbulent fluxes as seen in Grimmond et al. (2010) and therefore the turbulent intensity over and downwind of the urban area. Consequently, the impact of the urban area on the formation of the LLJ might vary depending on the surface properties of the urban area. However, an extensive analysis of the effect of urban surface properties on the LLJ is probably not yet feasible in PALM due to the expensive computation capacity needed for these simulations.

The PALM model uses cyclic-boundary conditions in our current experimental set-up. This is essential to allow the LLJ to develop within the model domain, where turbulence is largely resolved. However, this can result in a re-circulation of turbulence generated by the city at the inflow boundary. This effect is minimal for the $4 \mathrm{~km}$ concentric city experiment as turbulence dissipates before re-entering from the inflow boundary. However, for the $8 \mathrm{~km}$ and $12 \mathrm{~km}$ concentric cities, the advected turbulence re-enters from the inflow boundary, but is mainly directed towards the north part of the domain due to the change in wind direction. This can have implications for the LLJ formation over the domain as the recirculated turbulence can delay the LLJ formation and decrease its speed. Yet, given the change in wind direction, the short simulation time and the spatial extent of the domain $(30 \times 30 \mathrm{~km})$ we do not expect this to substantially affect the results of the simulations. A simulation with a $40 \times 30 \mathrm{~km}$ domain (additional $10 \mathrm{~km}$ in the x-direction) showed only slight difference compared to the concentric city $(30 \times 30 \mathrm{~km})$ experiment, which supports the previous assumption. An increase in the domain could resolved part of the re-circulation problem, but it would be computationally very expensive. Alternatively, the wind conditions could be provided at the boundaries through off-line nesting to COSMO model or to a coarser PALM domain, but this will lead to a LLJ generated in a coarser domain where turbulence is mainly parameterized.

In the PALM experiment spatially uniform initial profiles are used. These profiles are similar over both the urban and the rural area. Geostrophic wind speed is constant in time and there is no large-scale advection or subsidence for simplicity. In a realistic situation there would be differences in the initial forcing profiles between the urban and rural areas. Moreover, geostrophic wind speed might vary with time and there could be large-scale subsidence. These difference between the simplified and realistic atmospheric forcing can influence the LLJ formation. We tested the impact of time dependent geostrophic wind using the 1D-WRF model and found differences in the onset, duration and characteristics of the LLJ. Despite these differences the effect of increasing urban fraction on the LLJ remains similar as in the run with a time independent geostrophic wind.

Van de Wiel et al. (2010) showed that it is possible to have a backward inertial oscillation near the surface (20-40m a.g.l.), which can lead to a decrease in the near surface wind speed over the course of the night. In the PALM experiments we found a similar back- 
ward inertial oscillation occurring close to the surface, which reduced the near surface wind speed. This reduction in near-surface wind speed could have implications for heat stress and transport of pollutants away from urban areas. We recommended to further investigate the effects of this backwards inertial oscillation.

Banta et al. (2003) and Lundquist \& Mirocha (2008) showed that during nights with strong LLJ the strong downward turbulent mixing from the wind shear increases the TKE over time and can lead to the formation an "upside-down" boundary layer. In this study no evidence of a fully developed "upside-down" boundary layer is found. However, an increase of TKE with time is reported, which leads to a secondary peak in the TKE profile around $100 \mathrm{~m}$ above ground (Figures $5.3 .2 \mathrm{~b}, \mathrm{~d}$ ). This effect was more profound in the grassland and concentric city experiments, probably due to the higher LLJ speed and stronger wind shear in the nocturnal boundary-layer compared to the infinite city experiment.

\subsection{Conclusions}

Low-level jets are important phenomena that affect the ventilation of heat and pollutants in urban areas, and the turbulent exchange in the nocturnal UBL. The interactions between LLJs and urban areas, although previously reported, still requires further investigation. In this study we investigated the role of turbulent mixing, induced by the urban area, and momentum advection, from the surrounding countryside, during the initial stages of the LLJ formation. An idealised case study spanning a total of 4 hours (1700-2100 UTC 15 May 2019) is used, based on a real meteorological situation over London. Two different models, the 1D-WRF and the state-of-the-art PALM LES model, are used to investigate the role of urban fraction and city size on the LLJ formation.

Higher urban fraction leads to an increase in TKE during the evening transition, which delays the collapse of the daytime UBL and increases the turbulent mixing in the nocturnal UBL. Due to the increased turbulent mixing the onset of the LLJ is delayed up to 1.5 hours over the urban area, while the LLJ height increases (up to 100m) and the LLJ speed decreases (up to $2 \mathrm{~ms}^{-1}$ ). These results are in agreement with the findings from a rural (grassland) and an urban (infinite city) PALM experiments, despite some difference. However, when the city is surrounded by grassland (concentric city experiment), these effects on the LLJ formation are not located only over urban area but are present in the rural area downwind of the city.

In the concentric city PALM experiment the turbulence generated by the city delays the formation of a strong inversion over and downwind of the urban area. The increase in vertical mixing acts to delay the onset of the LLJ over and downwind of the city. Consequently, a strong momentum advection is initiated, due to the horizontal wind speed gradient between the city and the upwind rural area. The advection of momentum occurs 
shortly after the LLJ is formed over the upwind rural area and can contribute up to double the geostrophically-induced wind speed tendency at the initial stages of the LLJ formation. The importance of momentum advection on the LLJ formation over the city strongly depends on city size. The smaller city (4 km diameter) had minimal effects on the horizontal wind speed gradient and thus the contribution of momentum advection on the LLJ formation was minimal. The larger city (12 km diameter) caused substantial turbulent mixing, resulting in a LLJ formation dominated mainly by momentum advection.

These findings indicate that the turbulent mixing induced by the urban area delays the formation of the LLJ and causes a horizontal wind speed gradient, which results in the advection of the upwind rural LLJ over the urban area. These results are in agreement with previous findings of Barlow et al. (2015) for London. The LLJ formation over the rural areas downwind of the city is also affected by the advection of TKE originating from the urban area.

\section{Acknowledgements}

This research has been funded by the NWO grant no. 864.14.007. The authors would like to thank the ECMWF for providing operational analysis data used for 3D-WRF model initialisation and forcing, which is used to derive the forcing for the 1D-WRF and PALM LES models. Experiments and data analysis was performed on the high performance computing facility offered by the Dutch Science Organization NWO (grant number SH312-15). 
Chapter 6

Synthesis 
In this chapter, the results from the previous research chapters are synthesised and brought in context with current research efforts in the field of urban climate research. An in-depth discussion on the representation of important urban surface-UBL exchange processes is provided. Limitations of the current research approaches are discussed along, with potential solutions and future improvements. Finally, the key research findings from this thesis are utilised to propose future research recommendations.

\subsection{Internal and external uncertainty sources in the optimisation of urban surface parameters}

In chapter 2, we find that excluding the atmospheric feedback mechanisms between the surface and the overlying UBL can lead to a skewed surface parameter optimisation. Consequently, it appears necessary to test model sensitivity to changes in surface parameters using a coupled UCM-atmospheric model to circumvent this shortcoming. Unfortunately, that might not be an optimal solution. The results from chapter 3 indicate that model response to changes in external atmospheric forcing can be of a similar magnitude as the model response due to changes in surface parameters. Each approach has its own shortcomings, which might lead to erroneous optimisation of the prescribed surface parameters.

Note that chapter 2 and 3 should be viewed as an attempt to understand how the interactions between the urban surface and the overlying atmosphere are affect by changes in surface parameters or external atmospheric forcing. Therefore, the approaches followed in this thesis is not an attempt to optimize surface parameters. However, it might still be possible to combine both off-line and coupled UCMs to derive a more accurate optimisation for the surface parameters. This would nevertheless require a careful analysis of model sensitivity to changes in the external atmospheric forcing and surface parameters to identify potential compensation errors between surface-driven and atmospheric-driven model bias. Ideally, it would be preferred to avoid such a optimisation approach altogether and derive the surface parameters directly from observations of the urban surface properties when possible.

An interesting discussion point arising from the results of chapter 3 is the uncertainty in the observational forcing for the off-line UCM models. Although the atmospheric forcing is directly derived from observations, the measured radiation fluxes and turbulent exchange fluxes are not always representative for the same source area. The same might be true for the wind speed, temperature and humidity observations. Consequently, an attempt to optimise model performance already includes an existing uncertainty in atmospheric forcing, which needs to be investigated. Therefore, it might be of interest to utilise an ensemble approach for the atmospheric forcing in both off-line and on-line UCMs studies to be able to capture this uncertainty in atmospheric forcing and its effect on the surface 
energy balance.

\subsection{The role of advection in the turbulent exchange between the surface and the UBL}

As discussed in Chapter 1, advection of temperature and moisture often occurs over urban areas, either as an result of external synoptic and meso-scale flows (i.e. sea-breeze) (Lemonsu et al., 2006; Pigeon et al., 2007) or due to thermally induced circulations between urban and rural areas (Wang, 2009). The advection of air with different temperature and moisture has a profound impact on the surface energy balance and turbulent fluxes (Chapter 3). These results are in agreement with the findings of Pigeon et al. (2007) and Lemonsu et al. (2006). However, the effects of momentum advection are often overlook in the presence of heat and moisture advection. In Chapters 4 and 5 we find that horizontal momentum advection from rural to urban areas can be crucial for the formation of low-level jets (LLJs) over urban areas, with consequences for the turbulent exchange in the nocturnal UBL. Therefore, it is essential to study closely momentum advection processes over urban areas and their effect not only on temperature and UHI, but also on the turbulence in the UBL.

In Chapter 1 we introduced the terms of the urban surface energy balance and assumed that the effect of the horizontal advective flux is minimal. That might be true in homogeneous environments, where there are no sharp contrasts in temperature, moisture or wind speed. However, in a dense urban environment small-scale advective fluxes can affect the surface energy balance. This has previously been reported for London, where IBLs and advective fluxes from the Thames river can effect the energy balance in the King College measurement site (Kotthaus \& Grimmond, 2014a,b). These small-scale advective fluxes cannot be captured using coarse model resolutions as the heterogeneity is not resolved. This can lead to potential biases in the modelled surface energy balance. Moreover, small-scale advection can also occur within canyons. This is not captured when slab or single-layer UCMs are used. Multi-layer UCMs can compute advection within the urban canyon and therefore might be better at capturing small-scale advective fluxes, but they still cannot fully resolve the urban heterogeneity. To investigate the impact of advective fluxes on the surface energy balance in urban areas, we recommend to use very high resolution $(<10 \mathrm{~m})$ LES models for cities (i.e. Resler et al., 2017; Maronga et al., 2020) as these models can explicitly resolve the turbulence and surface heterogeneity.

\subsection{The benefits and pitfalls of case studies}

All research chapters of this thesis make use of short case-studies periods and thus it would be appropriate to discuss their utility for understanding interaction between the urban 
surface and the overlying atmosphere. Case studies are commonly used to study landatmosphere interactions and boundary-layer dynamics over both rural (Sterk et al., 2013; Bosveld et al., 2014b; Kleczek et al., 2014) and urban (Pigeon et al., 2007; Wang et al., 2011; Song \& Wang, 2016). They are usually short-term (1-2 day) period, during which the synoptic conditions are well known and the external atmospheric forcing on the surface can be accurately obtained via a use of observations and model simulations (Bosveld et al., 2014a; Steeneveld et al., 2017). Note that more control over the external forcing (i.e. exclusion of rainy days) allows for an easier identification of feedback mechanisms between the surface and the boundary-layer, and more precise quantification of their effect on the surface energy balance and the turbulent exchange.

The use a short case study period usually lacks the quantitative robustness of long-term model evaluations. This is the reason why off-line UCM studies often utilise relatively longer time-spans (Grimmond et al., 2010; Loridan et al., 2010). Yet, in coupled surfaceatmosphere simulations the use of long-term evaluation periods is very difficult as it requires accurate knowledge of external forcing during the case study period. For synoptic forcing and radiation that is somewhat trivial, but advection needs to be calculated from numerical model simulations, which are not particularly accurate over longer time periods ( $>7$ days). Another drawback of case studies, is the use of "golden days" (i.e. periods with clear skies). There is a point to be made that in urban environments the more extreme heat stress conditions occur during these clear-sky hot summer days. Nevertheless, they exclusions of days with clouds or rains limits the understanding of land-atmosphere interaction in the absence or strong temporal variability in the shortwave radiative forcing. Therefore, future research studies should also look to address the impact of surface parameters on the surface energy balance under less favourable weather situations. This will greatly help to develop a more robust understanding of the coupling between the urban surface and UBL.

\subsection{Atmospheric stability and radiative cooling over urban areas}

During the night the land-surface cools via the outgoing longwave radiation, in what is known as "radiative cooling". This results in the onset of stable atmospheric conditions, where potential temperature increases with height, and the formation of a stable nocturnal boundary layer (SBL) occurs. In the SBL there is little vertical mixing and high atmospheric stability, which can occasionally result in the de-coupling of the SBL flow from the surface (Mahrt, 1998; de Wiel et al., 2012; Steeneveld, 2014). This de-coupling can accelerate the "radiative-cooling" at the surface, which in turn can increase atmospheric stability (Mahrt, 1998; Walsh et al., 2008; Gentine et al., 2018). This stability-radiation feedback strongly affect the near surface temperature over rural areas. 
Over urban areas the nocturnal UBL is usually not as stable as its rural counterpart, due to larger surface roughness and heat released from the urban fabric during the night. Therefore, the interactions between atmospheric stability and radiative cooling over urban areas remains largely obscure. The results from Chapter 3 indicate that a feedback mechanism between atmospheric stability and radiation can result in faster surface cooling over cities. When the UBL warms up a lot during the day, it can lead to a larger surface-atmosphere temperature gradient at night, resulting in more stable atmospheric conditions and stronger radiative cooling. This feedback effect can be substantial over urban areas, but may not be as important as over rural areas, where it can lead to dramatic de-coupling and runway radiative cooling. Yet, it is nonetheless an interesting feedback to investigate further as it can have implications for the UHI and heat stress especially during heat waves.

Wind and meso-scale flows can affect the vertical mixing in the UBL and thus both affect the nocturnal atmospheric stability and surface cooling. In Chapters 4 and 5, we find that a LLJ can affect the turbulent mixing in the nocturnal UBL and consequently atmospheric stability. Similar finding have been reported by several other studies (Banta et al., 2003, 2006; Lundquist \& Mirocha, 2008; Barlow et al., 2015; Klein et al., 2016). The interactions between a LLJ and atmospheric stability in the nocturnal UBL has direct implications for the surface radiative cooling in urban areas. Namely, under strong LLJs radiative cooling from the urban surface can decrease due to weaker atmospheric stability in the UBL.

\subsection{Low-level jets: implications for heat stress and air quality over cities}

The investigation on the LLJ interactions with the UBL is mainly concerned with the effect of LLJs on the turbulent kinetic energy and vertical mixing within the nocturnal UBL. This scope, although interesting for boundary-layer meteorologists, does not address the effects that LLJs can have on the UHI, heat stress and air quality in cities.

$\mathrm{Hu}$ et al. (2013b) showed that UHI intensities over Oklahoma were lower under strong LLJs due to increased vertical mixing in the nocturnal boundary layer. The increased vertical mixing weakens the atmospheric stability and radiative cooling over rural areas, which leads to lower UHIs. Advection of cooler rural air can also be enhanced if the near-surface wind increases during nights with a LLJ. However, this might not always be the case as Van de Wiel et al. (2010) showed that during the LLJ formation a backward oscillation occurs near the surface, which reduces the near surface wind speed. This can result in a reduction of cold air advection. The interaction between LLJ, UHIs and heat advection can be very relevant for heat stress in urban areas. Therefore, it is likely that under strong LLJ conditions, the weaker stability and low near-surface wind decrease the 
surface radiative cooling and cold air advection resulting in higher temperatures and more heat stress.

LLJs have strong implications for air quality over urban areas. The increase in wind speed over the nocturnal UBL can increase the horizontal transport of pollutants away from urban areas. However, this is depends on the type of pollutant, its chemistry and depositions processes during the night. As seen in Hu et al. (2013b); Klein et al. (2014), the nocturnal near-surface ozone concentrations are elevated under strong LLJs, due to increased vertical mixing and entrainment of air with higher ozone concentrations from the residual layer. However, for PM2.5 Miao et al. (2019) found that under strong LLJ conditions, the deeper boundary layer depth results in lower concentrations of PM2.5. Therefore, the impact of LLJs on air quality in urban areas needs to be investigated for atmospheric pollutants that exhibit differences in their surface concentrations and vertical profiles between daytime and nighttime.

\subsection{The implementation of vegetation in UCMs: im- plications for the urban surface - UBL interac- tions}

Most UCMs, whether less or more complex, treat urban vegetation as a separate land-use type, with its own independent surface energy balance and surface fluxes (Chen et al., 2011a; Ward et al., 2016). Although these fluxes are averaged with the urban fluxes to compute the average surface fluxes from each model grid-cell, the vegetated surface processes are essential "semi-independent" from the processes in the urban land-use tile. This approach has implications for the representation of energy balance and turbulent exchange processes for both the urban canyon and the vegetated surface. Namely, the canyon temperature, moisture and wind is not taken into account during the computations of fluxes from the vegetation. Instead the vegetation fraction use these quantities from the 1st atmospheric model level. Moreover, there are no radiation interactions between trees and the wall/road facets (i.e. tree shading, multiplied reflections, long-wave radiation trapping).

Consequently, any analysis of model response to changes in urban surface parameters is only minimally affecting the vegetation via changes in entrainment and meteorological conditions at the first model level (Tsiringakis et al., 2019). Therefore, in most UCMs the coupling between urban surface, trees and the UBL is incomplete. Yet, recent progress in the UCM community resulted in the addition of urban canyon vegetation in both single-layer (Redon et al., 2020) and multi-layer (Krayenhoff et al., 2020) UCMs. The integrated canyon vegetation can improve the representation of radiation interaction within the canyon (i.e. shading effects), increase evaporative cooling, which can reduce heat stress, and decrease wind speeds due to increased canopy drag on the wind flow 
(Krayenhoff et al., 2020; Redon et al., 2020). This is a great step towards better weather forecasts at the street scale (Ronda et al., 2017).

\subsection{Advection of TKE and its role in accurate representation of boundary-layer downwind of cities}

In chapter 1 we introduced the differences in the vertical structure of the urban and rural boundary layers. These differences are not static in space. Under, moderate and strong winds the properties of the UBL can be advected downwind over rural areas (Barlow, 2014). This advection process can increase temperature in downwind rural areas (Heaviside et al., 2015; Bassett et al., 2017, 2019). These advection process are captured by all coupled UCM-3D atmospheric models that use an urban tile and can have substantial implications for the boundary-layer structure downwind of urban areas.

One advection effect that is not well represented in current 3D-atmospheric models is the horizontal advection of turbulent kinetic energy (TKE), because TKE is usually parameterized depending on the existing conditions in the column/grid-cell in which it is quantified. This is not the case when one uses LES or DNS models as these explicitly resolve turbulence. Yet, some atmospheric models can parametrize the horizontal advection of TKE (Olson et al., 2019). As seen in Kalverla et al. (2019a) and in Chapters 4 and 5, the advection of TKE downwind of the urban area is essential for the correct representation of boundary-layer turbulence and depth. This has substantial implications also for the formation of LLJs over the rural areas downwind of cities. In chapter 5, we present evidence that in the downwind advected UBL plume, the formation of LLJ is substantially delayed due to increased vertical mixing. This highlights the need to include horizontal advection TKE processes in our current atmospheric models to be able to accurately capture the boundary layer and its interactions with meso-scale flow downwind of cities.

\subsection{A future for urban climate research}

In a world of ever increasing computational power it is only logical to strive for a much finer horizontal resolution in our weather forecasts models. This will eventually allow us to resolve explicitly the two "Holy Grails" of urban meteorology, the urban heterogeneity and turbulent transport in urban areas. Consequently, a much better understanding of the interaction between the urban surface and the urban boundary-layer and their effects on the various meso-scale flows can be achieved. Explicit quantification of heat stress at the street level with also be possible, as we no longer have to rely on inadequate parameterization of urban canyon process and their inherit biases. Human exposure to 
atmospheric pollutants at the street level will also be simulated and fully coupled with the atmospheric flow at the canyon as seen in Kurppa et al. (2019).

However, this future does not come without challenges. To utilise these type of models to their full potential, an accurate description of all urban surface properties and morphological characteristics needs to be provided, ideally at horizontal and vertical resolution of a few meters. And even when these criteria have been met, the simulation will be exceptionally time-consuming and thus prohibiting for an effective weather forecast. For the moment, these new modelling tools will be primarily directed towards research and not operational meteorology.

Therefore, it is not the time yet to abandon our previous UCMs that so admirably help us to parametrize processes in the urban environment for a very long time. Actually, the current developments in many of these UCMs (Ward et al., 2016; Redon et al., 2020; Krayenhoff et al., 2020) make them ideal tools both for research and weather forecast in urban areas. Moreover, via the use of CFD modelling of urban environments, new and updated UCMs can be developed (Buccolieri et al.). Hopefully, these new UCMs can overcome existing limitations of older UCMs, like flux calculations from vertical surfaces, where Monin-Obukhov theory is still applied in many UCMs.

The representation of urban heterogeneity in UCMs can be further improved by an ever increasing supply of local climate zone maps (i.e. WUDAPT datasets) that provide information for the urban surface morphology and parameters in many cities across the world (Ching et al., 2018; Demuzere et al., 2019). This will allow UCMs to provide improved weather and air quality forecasts in urban areas, even for developing countries, where details urban surface morphological parameters are not widely available (Demuzere et al., 2017).

To conclude, there is more than enough room for all type of UCMs in the foreseeable future, be it simple or more complex. More complex multi-layer UCMs can slowly take the place of single-layer UCMs in weather prediction and research, especially for street-level weather forecasts. Single-layer and slab UCMs are eventually being implemented in climate models, as their parameterization of urban surface process can be important for the accurate prediction of future climate change (Katzfey et al., 2020). In the context of these developments, relevant urban climatic information can be provided to city services/urban planner, which can improve city-wide climate change adaptations efforts Masson et al. (2020). The numerous developments in the field of urban climate research indicate that a bright future is ahead for both modelling and observing weather in urban areas. 
Appendix 


\section{A.1 Chapter 2: Supplementary material}

Figures A.1.1, A.1.2, A.1.3 and A.1.4 with their corresponding captions. The figures show the variation of short-wave and long-wave radiations components as a function of changes in the surface parameters.
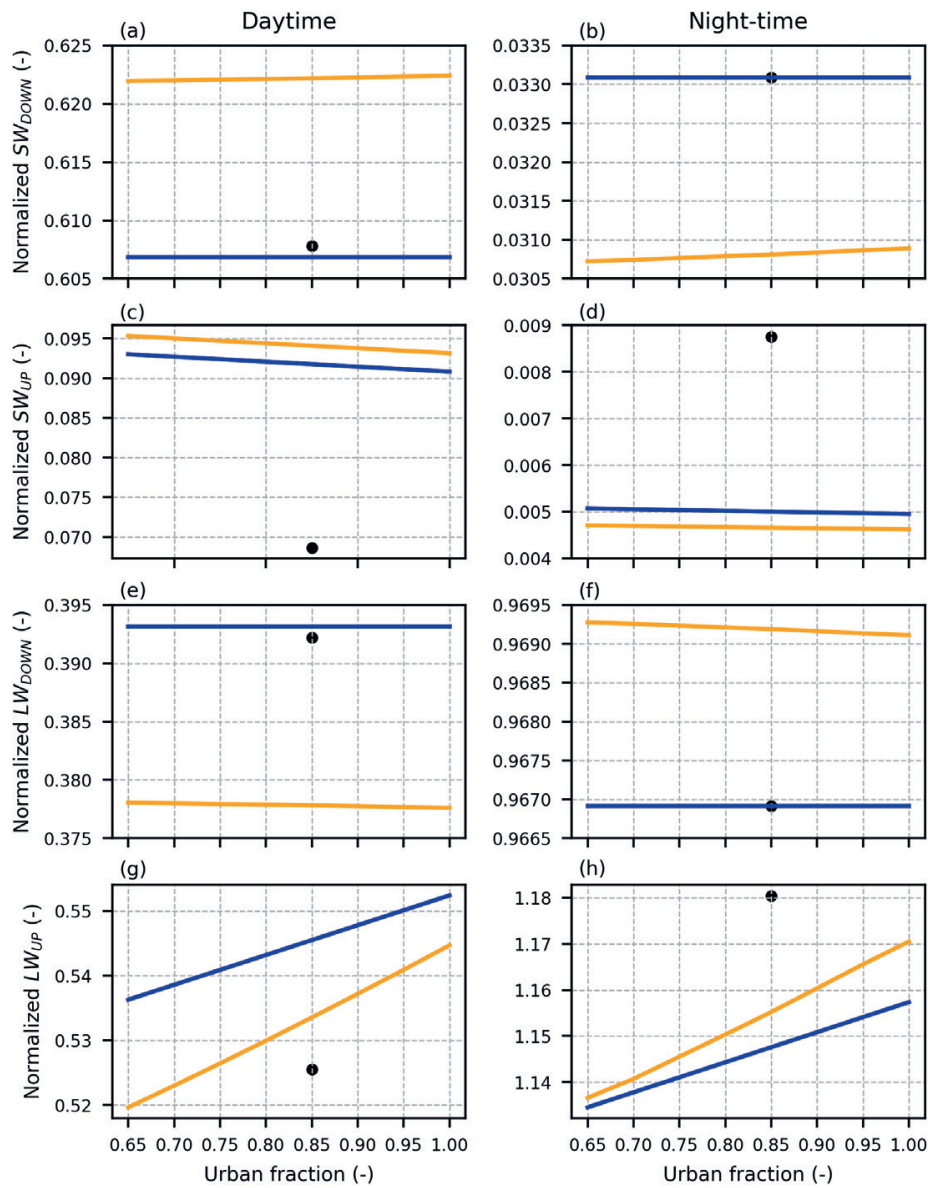

on-line off-line

- observations

Figure A.1.1: Normalized $S W_{D O W N}(\mathrm{a}, \mathrm{b}), S W_{U P}(\mathrm{c}, \mathrm{d}), L W_{D O W N}(\mathrm{e}, \mathrm{f})$ and $L W_{U P}(\mathrm{~g}, \mathrm{~h})$ for daytime (left) and night-time (right) as a function of the urban fraction for on-line (orange) and off-line (blue) cases. Modelled and observed fluxes are study period means (0600 UTC 23 July 2012 to 0600 UTC 25 July 2012). 

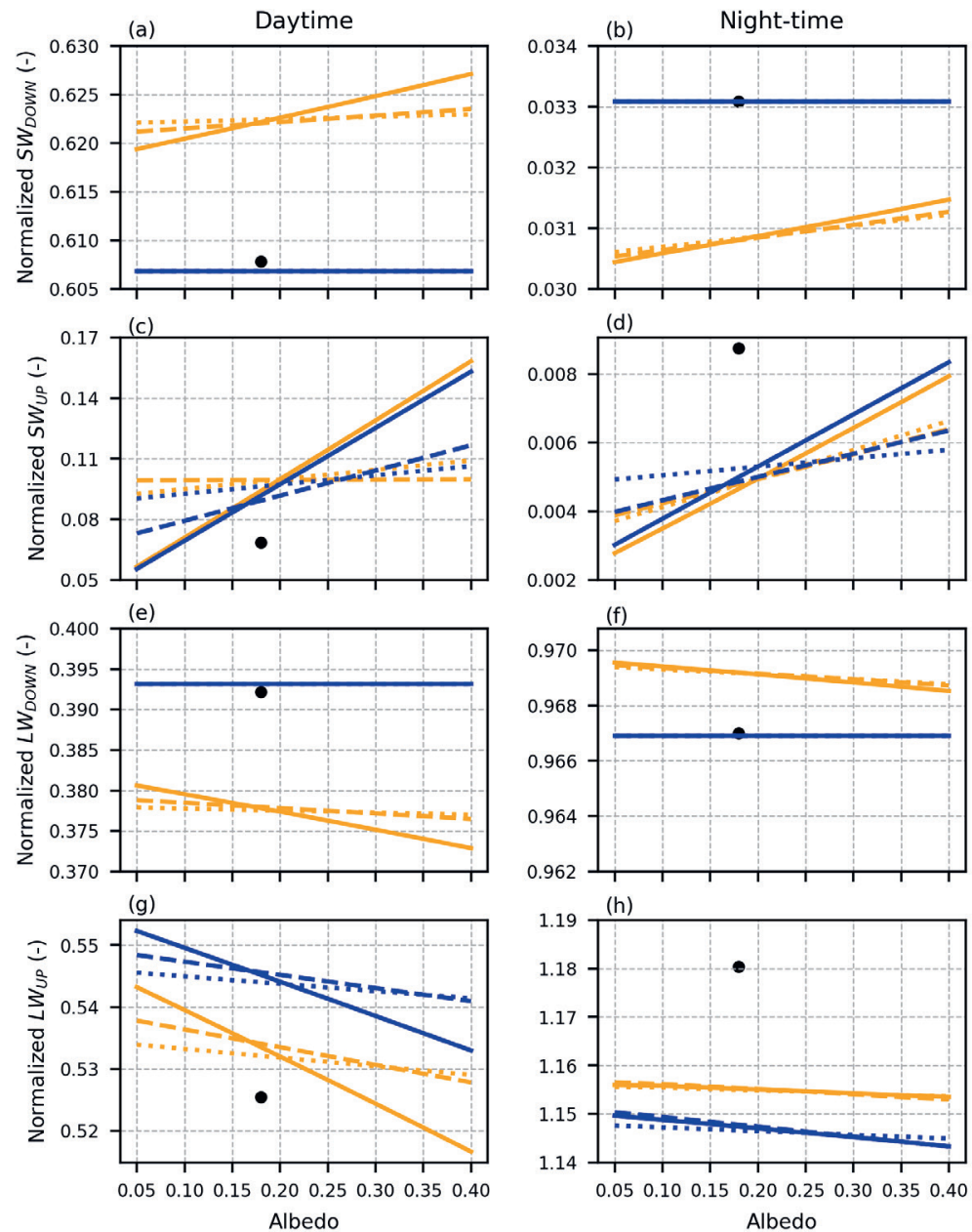

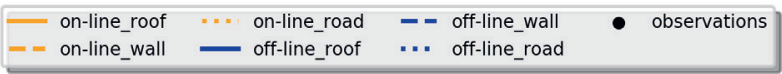

Figure A.1.2: As Figure A.1, but for facets' albedo. 

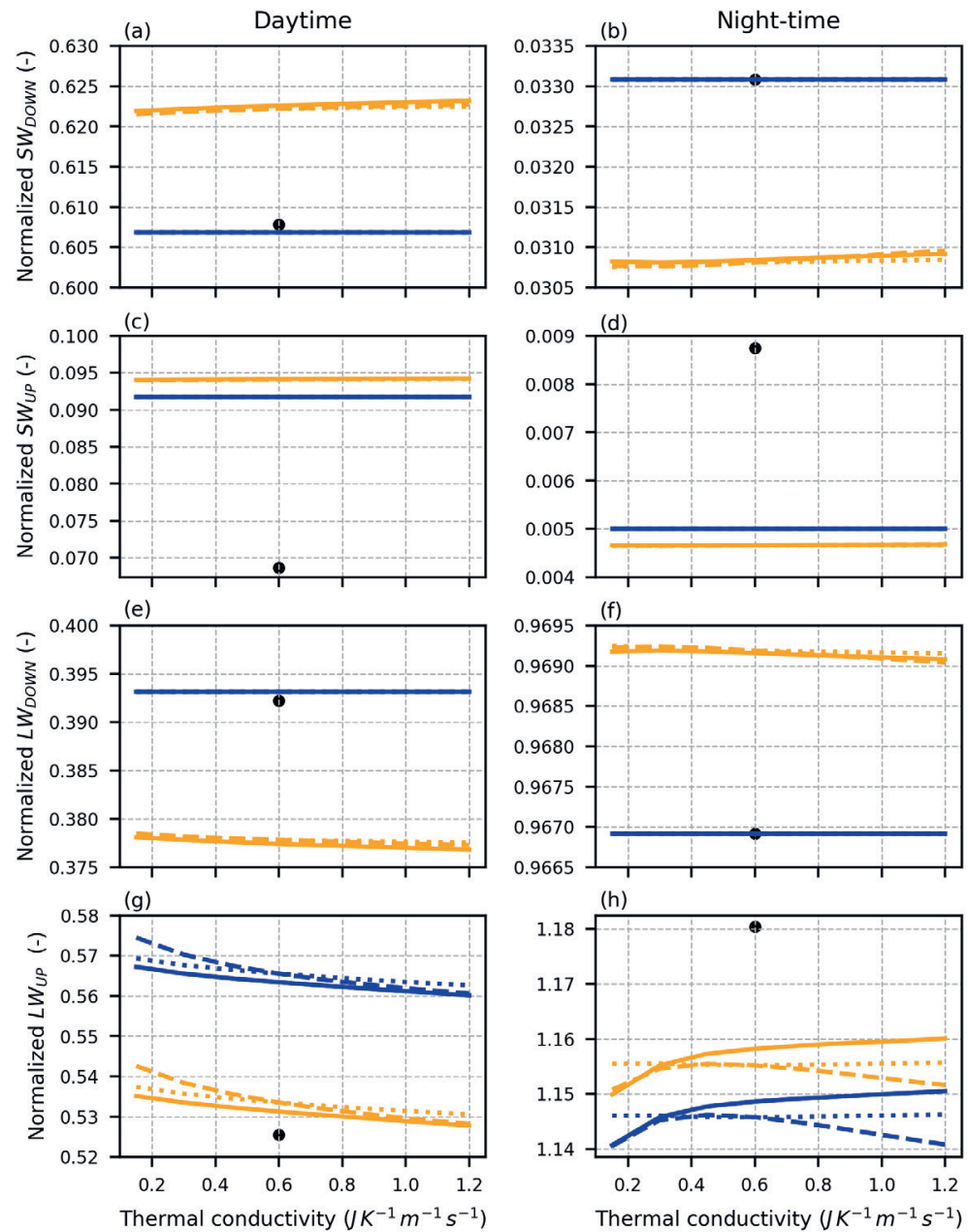

\begin{tabular}{|c|c|c|c|}
\hline $\begin{array}{l}\text { _- on-line_roof } \\
\text { - on-line_wall }\end{array}$ & $\begin{array}{l}\text { on-line_road } \\
\text { off-line_roof }\end{array}$ & $\begin{array}{l}-- \text { off-line_wall } \\
\ldots \text { off-line_road }\end{array}$ & - observations \\
\hline
\end{tabular}

Figure A.1.3: As Figure A.1, but for facets' thermal conductivity. 

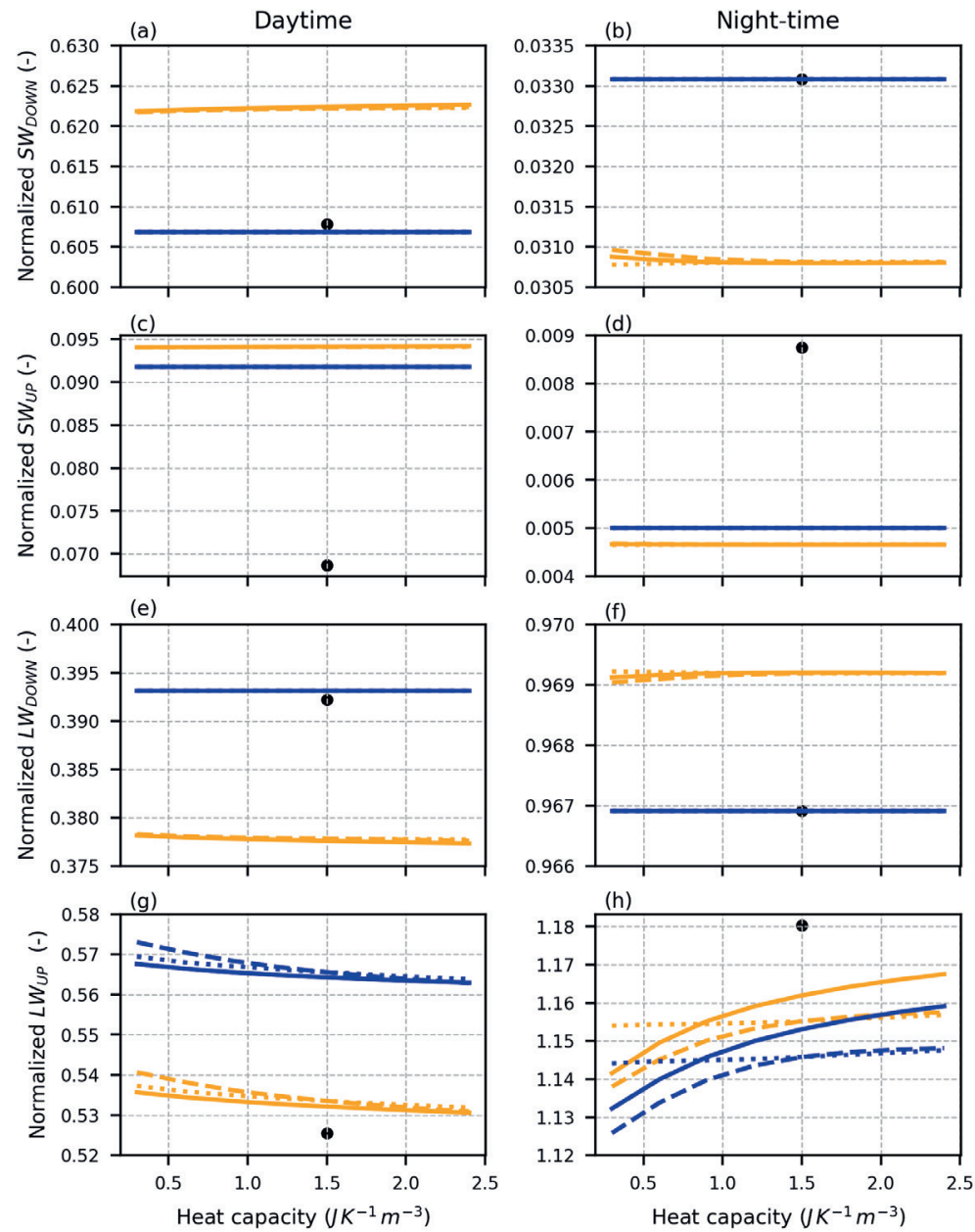

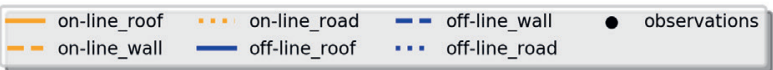

Figure A.1.4: As Figure A.1, but for facets' heat capacity. 


\section{A.2 Chapter 3: Definition for symbols}

Table A.2.1: List of definitions for the symbols/abbreviations used to define atmospheric forcing components, surface parameters, tested parameterisation schemes and other relevant variables.

\begin{tabular}{|c|c|}
\hline Symbol & Definition \\
\hline$a_{\text {kanda }}$ & Kanda parameter \\
\hline$a_{\text {roof }}$ & Roof albedo \\
\hline$A D V_{\theta}$ & Advection of potential temperature \\
\hline$A D V_{q}$ & Advection of moisture \\
\hline$A O D$ & Aerosol optical depth \\
\hline$B E P$ & Building environment parameterisation (Martilli et al., 2002) \\
\hline$B E P+B E M$ & BEP + Building Energy Model (Salamanca \& Martilli, 2009) \\
\hline$C A M_{s w}$ & CAM shortwave radiation scheme (Collins et al., 2004) \\
\hline$C A M_{l w}$ & CAM longwave radiation scheme (Collins et al., 2004) \\
\hline $\mathrm{CO}_{2}$ & Concentrations of $\mathrm{CO}_{2}$ in ppm \\
\hline $\mathrm{CH}_{\text {heat }}$ & Exchange coefficient of heat \\
\hline $\mathrm{CH}_{\text {mom }}$ & Exchange coefficient of momentum \\
\hline$C_{\text {wall }}$ & Heat capacity of walls \\
\hline$\Delta Q_{s}$ & Storage heat flux \\
\hline$\Delta T_{50 m}$ & Difference between $T_{50 m, \max }$ and $T_{50 m, \min }$ \\
\hline$\Delta T_{\text {skin }}$ & Difference between $T_{\text {skin,max }}$ and $T_{\text {skin,min }}$ \\
\hline$f_{\text {urban }}$ & Urban fraction \\
\hline$\lambda_{\text {wall }}$ & Thermal conductivity of walls \\
\hline$L W_{\text {down }}$ & Long-wave downward radiation \\
\hline$L W_{u p}$ & Long-wave upward radiation \\
\hline$M Y J$ & Mellor-Yamada-Janijc boundary layer scheme (Janjic, 1994) \\
\hline$Q_{f}$ & Anthropogenic heat flux \\
\hline$Q^{*}$ & Net all-wave radiation \\
\hline$Q_{H}$ & Sensible heat flux \\
\hline$Q_{E}$ & Latent heat flux \\
\hline QNSE & Quasi-normal Scale Elimination boundary layer scheme (Sukoriansky et al., 2005) \\
\hline$S W_{\text {down }}$ & Short-wave downward radiation \\
\hline$S W_{u p}$ & Short-wave upward radiation \\
\hline$S L U C M$ & Single-layer urban canopy model (Kusaka et al., 2001) \\
\hline$Y S U$ & Yonsei university boundary layer scheme (Hong et al., 2006) \\
\hline
\end{tabular}




\section{A.3 Chapter 3: Supplementary material}

This supporting information provides supplementary figures and further analysis to support/enhance the conclusions of the chapter. Data and figures presented here are following the same methodology as indicated in the chapter.

\section{A.3.1 Evaluation of surface shortwave and longwave radiation}

The modelled vs observed short-wave down $\left(S W_{\text {down }}\right)$, short-wave up $\left(S W_{u p}\right)$, long-wave down $\left(L W_{\text {down }}\right)$ and long-wave up $\left(L W_{u p}\right)$ radiation are compared in Figure A.3.1.
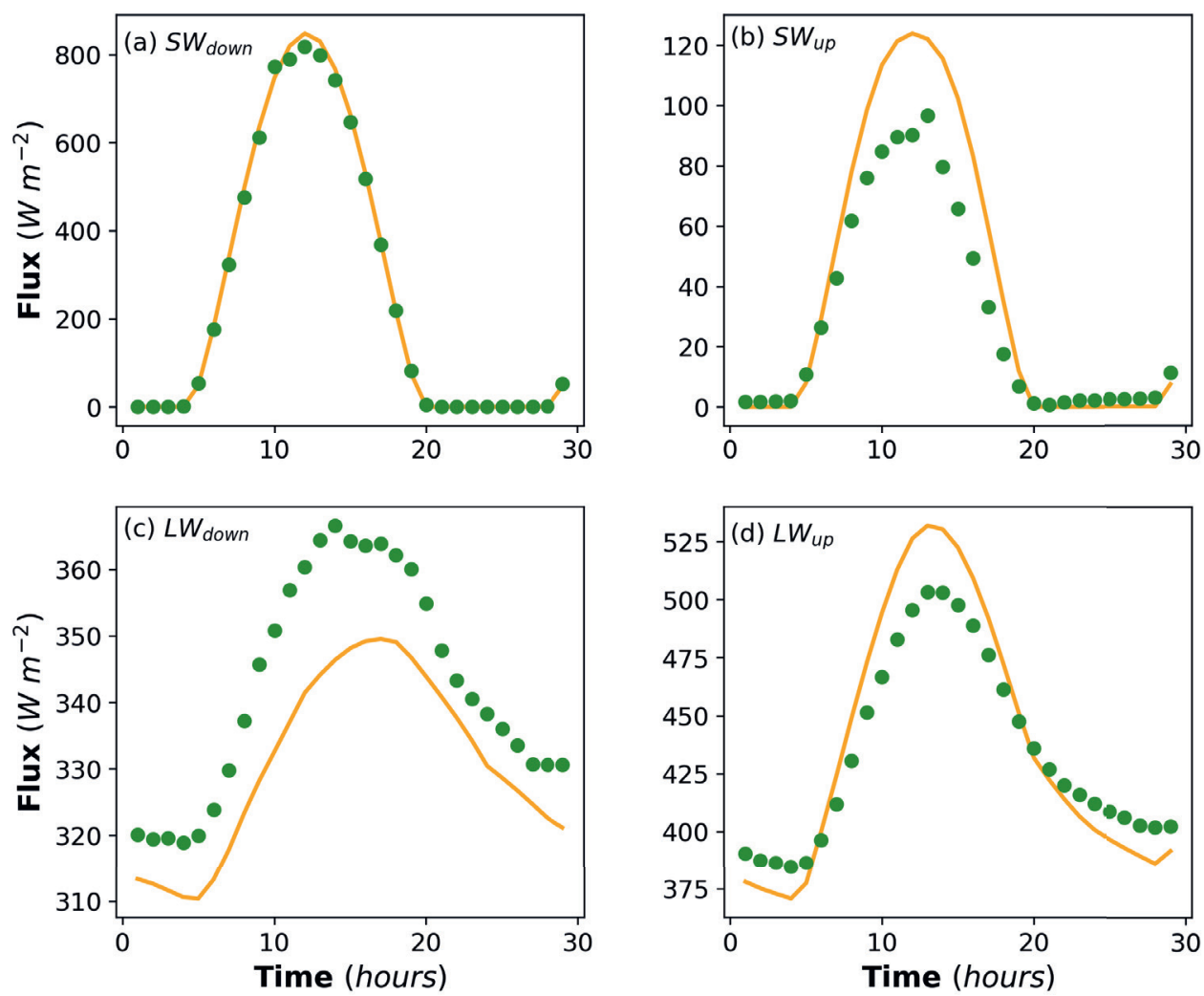

WRF-SLUCM-Noah

- observations

Figure A.3.1: As Figure 3.3.1, but for (a) short-wave down $\left(S W_{\text {down }}\right)$, (b) short-wave up $\left(S W_{u p}\right)$, (c) long-wave down $\left(L W_{\text {down }}\right)$ and (d) long-wave up $\left(L W_{u p}\right)$ radiation components $\left(W m^{-2}\right)$. 


\section{A.3.2 Day and Night model sensitivity for the second case study day}

The following results are for the second day (0600 UTC 24 July 2012 to 0500 UTC 25 July 2012) of simulations using the methodology outlined in chapter 3. Although, the two days are treated separately in these analyses they obviously come from a common model run.

\section{A.3.3 Surface Radiation Balance}

The response of the daytime modelled shortwave downward $\left(S W_{\text {down }}\right)$ and net all-wave radiation $\left(Q^{*}\right)$ remains the same between two days. Changes in atmospheric forcing (Figure 3.3.2a, A.3.2a) have the same orientation in the variable space, but the bias between the modelled $Q^{*}$ and observed $Q^{*}$ is $10 \mathrm{Wm}^{-2}$ smaller in the second day. This decreases the $Q^{*}$ bias (from -43.6 to $-30 W^{-2}$ ). However, the $\left(S W_{\text {down }}\right)$ radiation bias increases (from 13.6 to $22.5 \mathrm{Wm}^{-2}$ ), potentially linked to an increase in AOD and a small bias in simulated boundary-layer moisture. Surface parameter changes affect the modelled $\left(S W_{\text {down }}\right)$ and $\left(Q^{*}\right)$ similarly on both days (Figure 3.3.2b, A.3.2b) and explanations of the bias remain the same (i.e. AOD and $a_{\text {roof }}$ expected to reduce model bias).
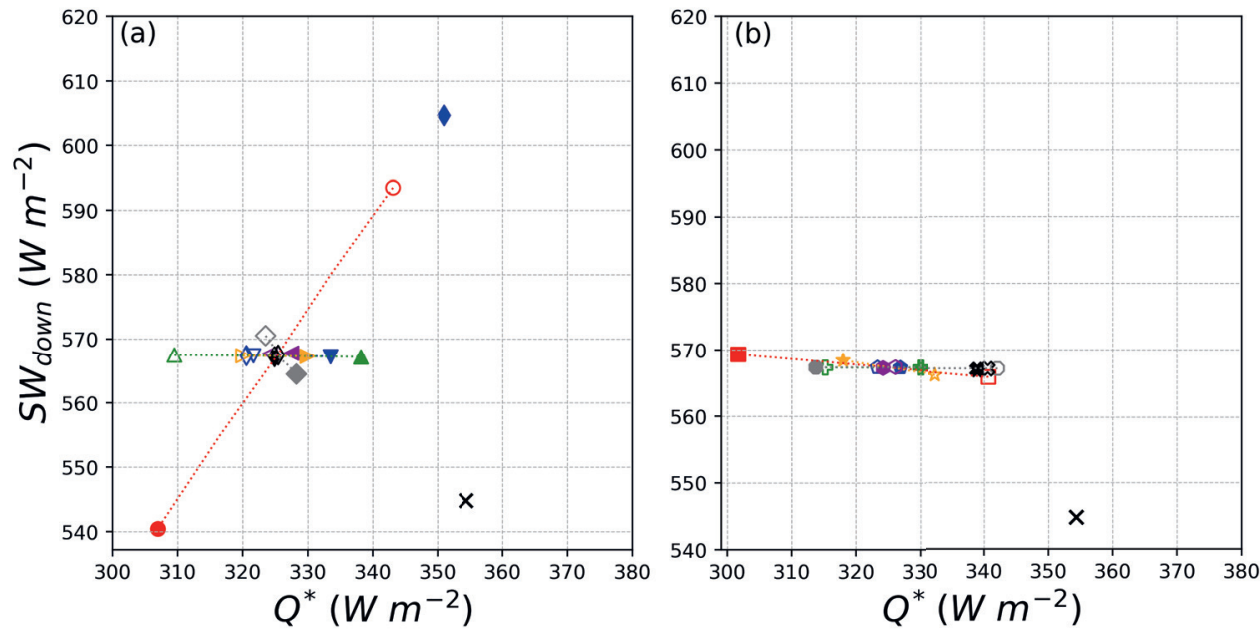

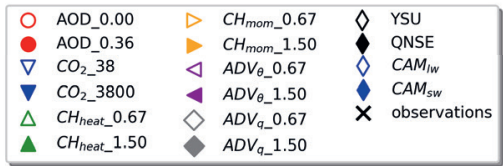

\begin{tabular}{|c|c|c|c|c|c|}
\hline$\square$ & $a_{\text {roor_}} 0.10$ & $\$$ & $\lambda_{\text {wall_}} 1.05$ & 0 & $a_{\text {kanda__}} 0.50$ \\
\hline$\square$ & $a_{\text {roor_}} 0.30$ & $\Delta$ & $f_{\text {urb_} \_} 0.75$ & - & $a_{\text {kanda_1.40 }}$ \\
\hline 0 & $C_{\text {wall_}} 0.6$ & $\star$ & $f_{\text {urb_o }} 0.95$ & $\mathfrak{\Sigma}$ & BEP \\
\hline 슴 & $C_{\text {wall_}} 2.4$ & 0 & $Q_{f_{-}} 10$ & * & $\mathrm{BEP}+\mathrm{BEM}$ \\
\hline \& & $\lambda_{\text {wall_}} 0.15$ & 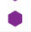 & $Q_{f_{-}} 70$ & $x$ & observations \\
\hline
\end{tabular}

Figure A.3.2: As Figure 3.3.2, but for the period 0600 UTC 24 July 2012 to 1800 UTC 24 July 2012.

Daytime sensitivity of $\Delta T_{50 \mathrm{~m}}$ is similar for both days (Figure 3.3.3a,b, A.3.3a,b), with 
few exceptions. Decreasing AOD leads to a smaller $\Delta T_{50 m}$ (by $0.5 \mathrm{~K}$ compared to $0.1 \mathrm{~K}$ the first day), but the sensitivity range of $Q^{*}$ remains the same. Changes in $Q_{f}$ have a non-linear effect on $\Delta T_{50 m}$ during the second day (Figure A3.4b), but the total variation of $\Delta T_{50 m}$ remains small $(0.4 \mathrm{~K})$.
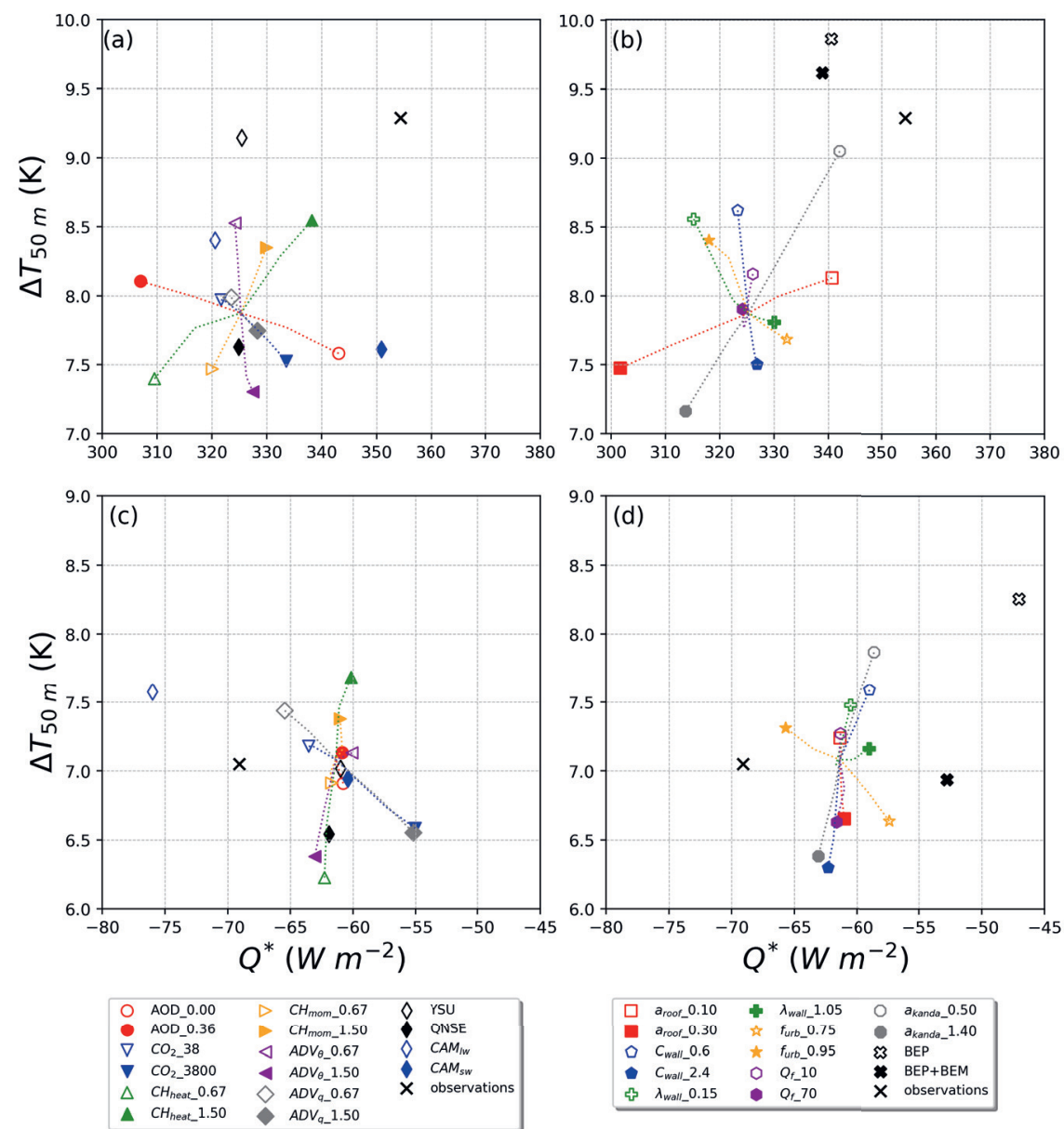

Figure A.3.3: As Figure 3.3.3, but for the period 0600 UTC 24 July 2012 to 0500 UTC 25 July 2012 .

A non-linear response to changes in $Q_{f}$ on the second day. On the first day an increase in $Q_{f}$ slightly increases daytime temperature range $(0.1 \mathrm{~K})$, whereas the next has nearly no impact on $\Delta T_{50 m}$. However, the decrease in $Q_{f}$ increases daytime $\Delta T_{50 m}$ (by $0.2 \mathrm{~K}$ ). This is partially explained by the prior night's smaller temperature decrease (Figure S3d) when high $Q_{f}$ values are prescribed. Additionally, a lack of subsidence on day 2 causes a larger proportion of the $Q_{f}$ increase to contribute to increasing the boundary-layer 
height (rather than increasing the $\Delta T_{50 m}$ ) and the much lower wind speed also impacts the boundary-layer dynamics (discussed section A.3).

The modelled sensitivity for both nights is rather similar, with the exceptions of the model response on advection of potential temperature $\left(A D V_{\theta}\right)$ and changes to $f_{\text {urb }}$ (Figures A.3.4c,d and A.3.5c,d). Increase in $A D V_{\theta}$ now leads to a smaller night-time $\Delta T_{50 m}$ $(6.4 \mathrm{~K})$, while decreasing $A D V_{\theta}$ results in the same night-time $\Delta T_{50 \mathrm{~m}}(7.1 \mathrm{~K})$ as the default model run. The difference in the modelled $\Delta T_{50 m}$ response to increasing $A D V_{\theta}$ shows a non-linear response due to a non-linear atmospheric feedback. When strong $A D V_{\theta}$ is prescribed, daytime $T_{50 m}$ decreases leading to a smaller nocturnal surface-to-air temperature gradient and weaker atmospheric stability compared to runs with weaker advection. Weaker stability leads to a deeper boundary-layer (not shown), where de-coupling between the surface and the overlying atmosphere occurs less often due to more vertical mixing (discussed in section A.3). Thus, radiative cooling is spread over a deeper nocturnal boundary-layer leading to smaller changes in $\Delta T_{50 m}$. This non-linear feedback affects nocturnal $\Delta T_{50 m}$ during the first night, but due to high wind speed and more shear it is not as strong. The same non-linear feedback explains the increase in night-time $\Delta T_{50 m}$, when $f_{u r b}$ increases. The increases in the sensitivity ranges of the nocturnal $\Delta T_{50 m}$ and $Q^{*}$ from changes in $A D V_{q}$, is caused by the larger amount of positive $A D V_{q}$ prescribed during the second day.

\section{A.3.4 Energy partitioning}

The modelled surface energy flux ratio $\left(Q_{H} / \Delta Q_{s}\right)$ difference in sensitivity between the day 1 and day 2 of the case study is similar (Figures 3.3.4 and A.3.4). During the day the sensitivity lines show identical variable space orientations, but magnitude changes occur for $A D V_{\theta}, Q_{f}$ and $c_{\text {wall }}$. The reference run $Q_{H} / \Delta Q_{s}$ decrease on day 2 (from 1.55 to 1.40 ) associated with the decrease in $Q^{*}$.

Although the nocturnal response to surface parameter and atmospheric forcing is nonlinear it does not lead to a substantial difference in model response between the two days. The modelled nocturnal $\Delta Q_{s}$ sensitivity remains similar for both nights (Figure 3.3.5 and A.3.5) with noticeable differences only in the response of nocturnal $\Delta T_{50 m}$. 

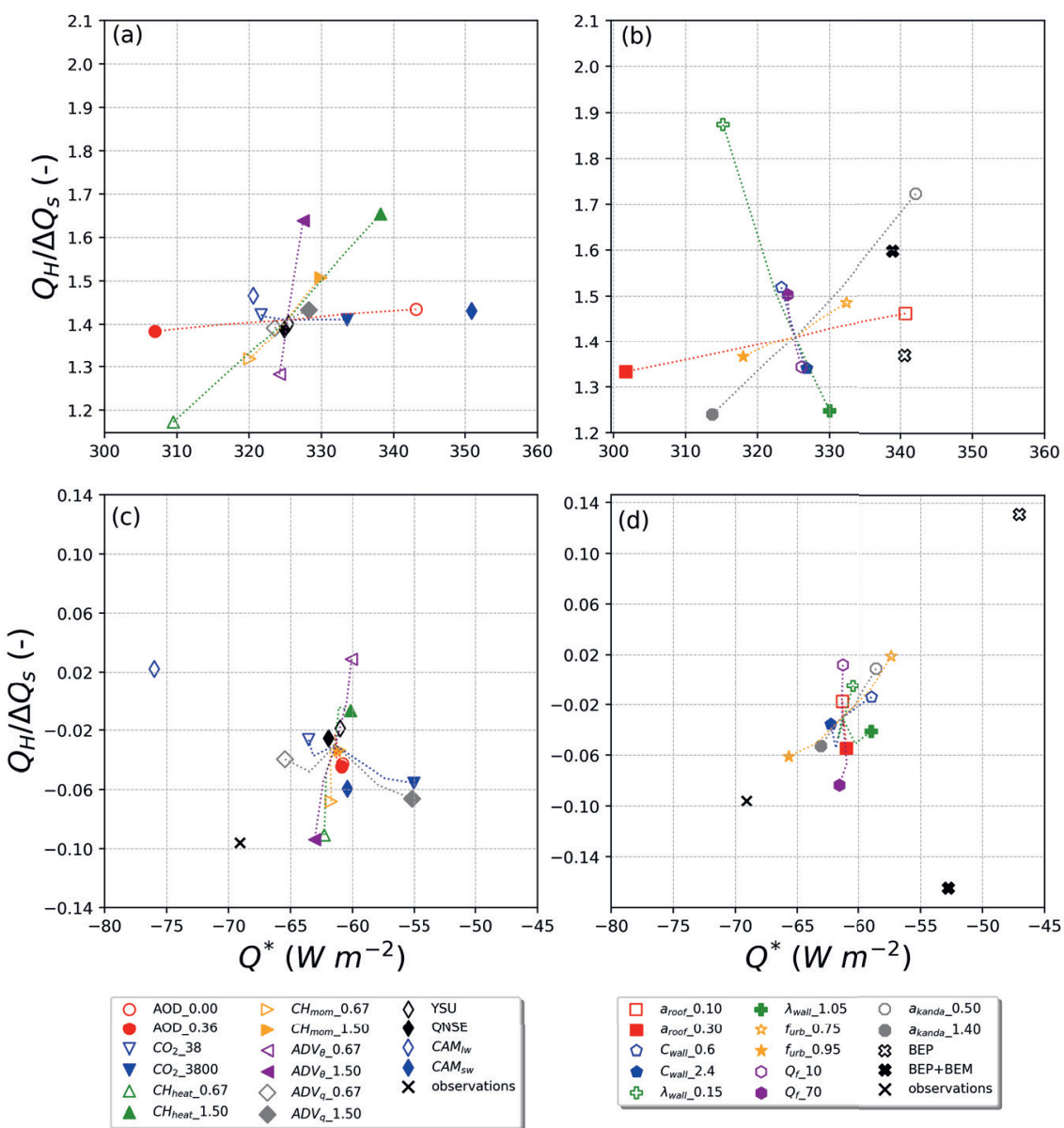

Figure A.3.4: As Figure 3.3.4 but for the period 0600 UTC 24 July 2012 to 0500 UTC 25 July 2012.

\section{A.3.5 Intensity of turbulent mixing}

Most of the differences in the model responses to changing surface parameters and atmospheric forcing originate from the effects they have on the near surface atmospheric stability (Figures 3.3.6 and A.3.6). The lower wind speed during the second day leads to more daytime instability $\left(R i_{B}\right.$ of -2.64 , cf. - 0.64$)$. Atmospheric stability increases at night $\left(R i_{B} 1.42\right.$ cf 0.19$)$ due to lower wind shear larger radiative cooling and increased $\Delta T_{50 m}$ (Figure A.3.6c,d). This difference also explains the increase in model variability during the second day, as the lower wind shear near the surface will increase the impact of near surface temperature gradient in atmospheric stability. Despite these difference in 

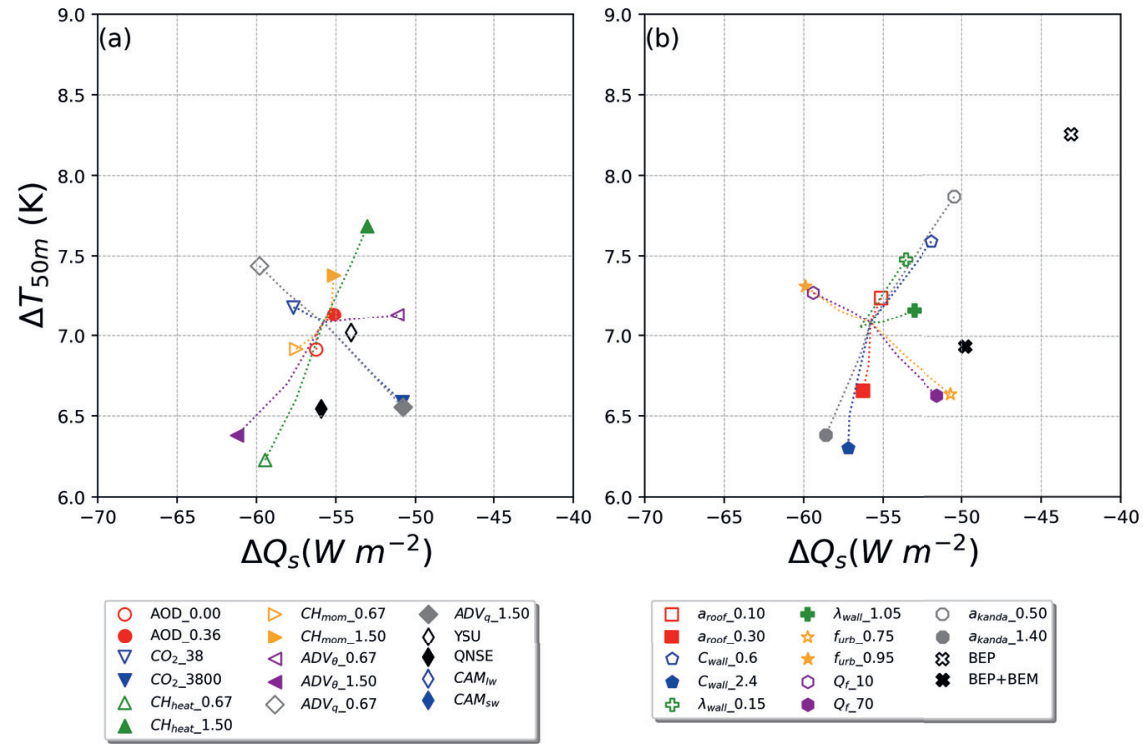

Figure A.3.5: As Figure 3.3.5 but for the period 1800 UTC 24 July 2012 to 0500 UTC 25 July 2012 .

magnitude, there is no difference orientation of sensitivity lines (Figures A.3.6a,b).

The nocturnal modelled $R i_{B}$ changes with atmospheric forcing and surface parameters become non-linear, increasing model sensitivity for some components with very small sensitivity during the first night. This enhanced $R i_{B}$ response is linked directly to the radiative cooling.

\section{A.3.6 Hourly model sensitivity}

The temporal evolution of the modeled sensitivity range of $Q^{*}$ and $T_{50 m}$ are similar on both days (Figure A.3.8), with non-linear atmospheric feedback is evident at night.

The change in $A D V_{\theta}$ sensitivity range of modeled $T_{50 m}$ decreases after 23 UTC for both days (Figures A.3.7, A.3.8, A.3.9). The decrease is larger on day 2, associated with the non-linear feedback to the near surface atmospheric stability (Figure A.3.10). Similar response is found for changes in $Q_{f}, f_{u} r b, C H_{\text {heat }}, a_{\text {kanda }}$ and $a_{\text {roof }}$. The low wind conditions (day 2) effects on near surface stability are amplified (Figure A.3.10), increasing the nocturnal $T_{50 m}$. 

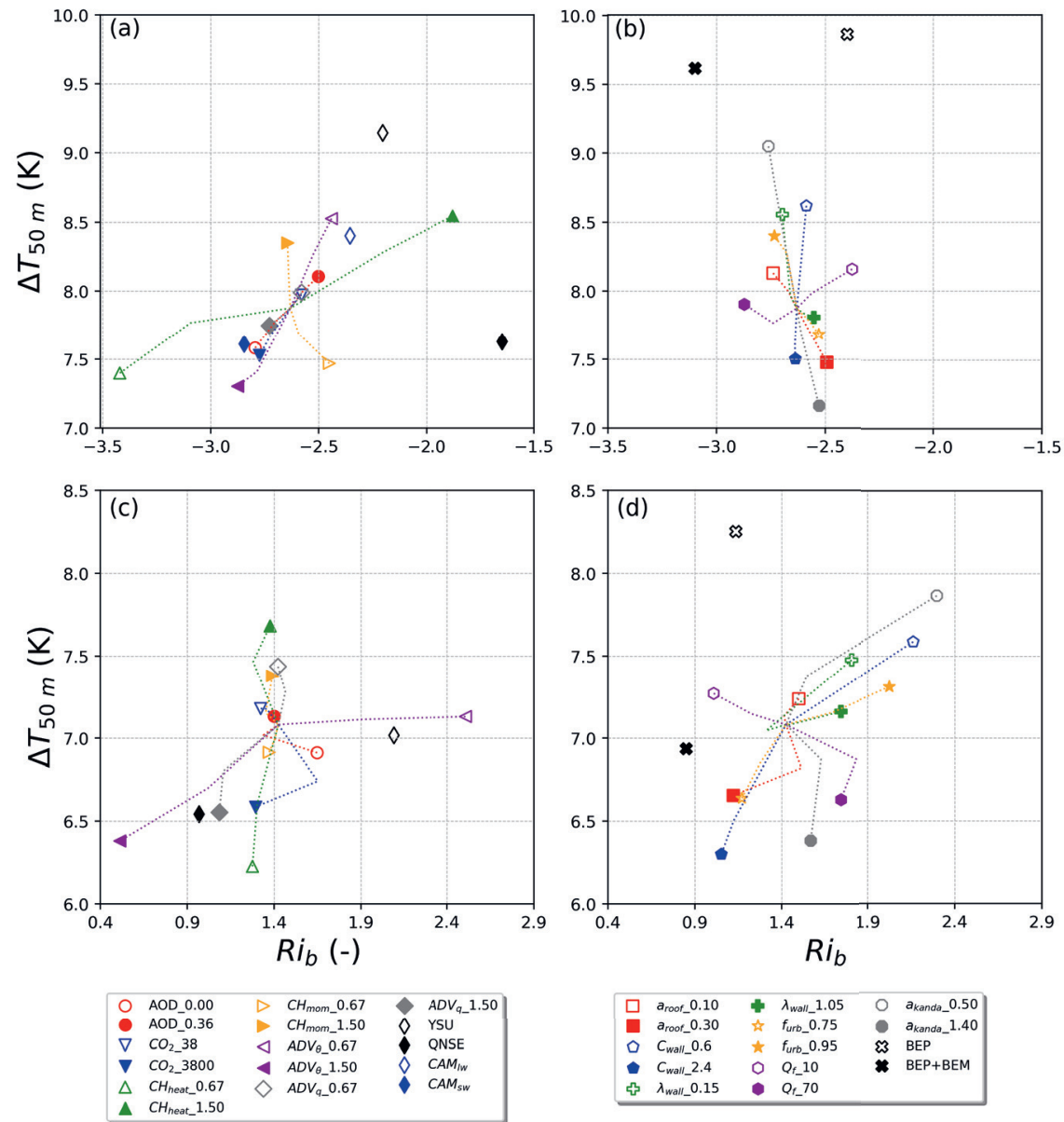

Figure A.3.6: As Figure 3.3.6 but for the period 0600 UTC 24 July 2012 to 0500 UTC 25 July 2012. 
(a)

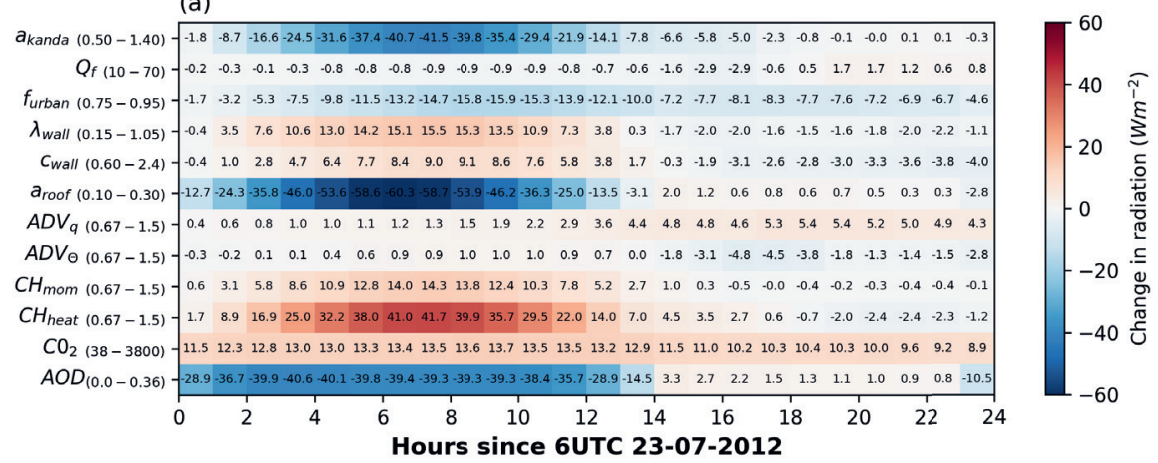

(b)

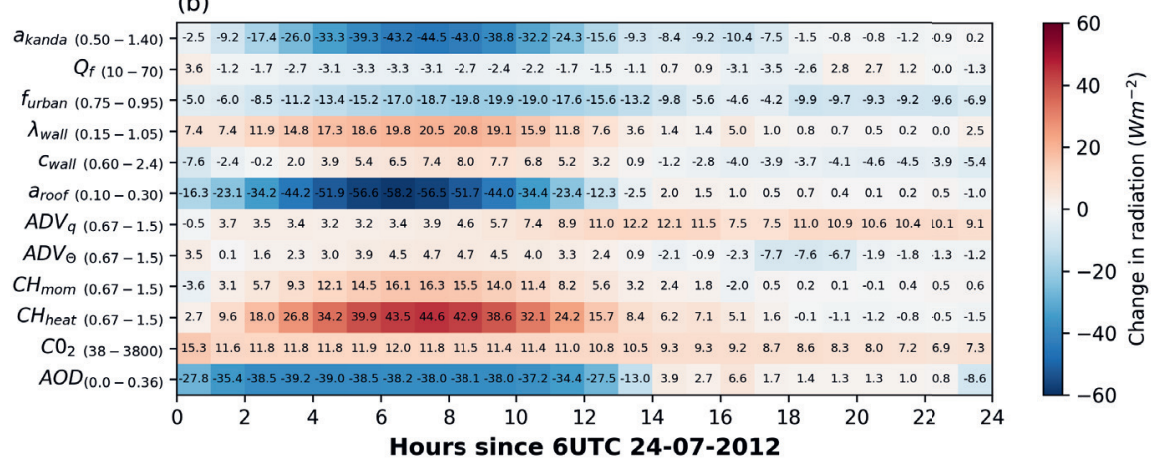

Figure A.3.7: Hourly evolution of the difference in net all-wave radiation $\left(Q^{*}\right)$ between runs using the maximum and minimum values for surface parameters and atmospheric forcing (Table 3.3.1, 3.3.2) for the first (a) and second day (b) of the case study period 
(a)

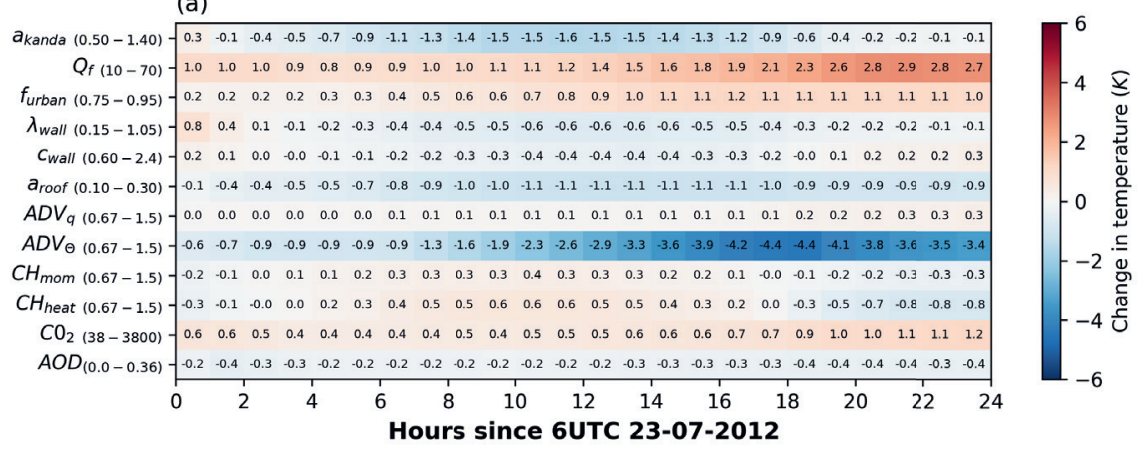

(b)

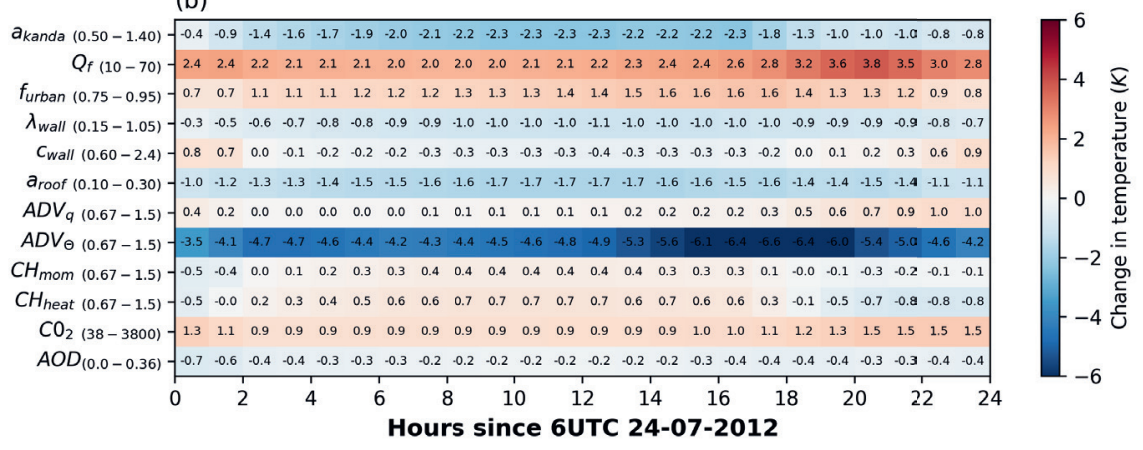

Figure A.3.8: As Figure A.3.7, but for the $50 \mathrm{~m}$ temperature $\left(T_{50 \mathrm{~m}}\right)$ 
(a)

\begin{tabular}{|c|c|c|c|c|c|c|c|c|c|c|c|c|c|c|c|c|c|c|c|c|c|c|c|c|}
\hline \multirow[b]{2}{*}{$a_{\text {kanda }}(0.50-1.40)$} & \multicolumn{23}{|l|}{ (u) } & \multirow{2}{*}{1.00} \\
\hline & -0.5 & -0.2 & -0.1 & -0.2 & -0.2 & -0.2 & $-0.2-c$ & -0.1 & -0.1 & -0.0 & -0.0 & 0.0 & 0.1 & 0.1 & 0.1 & 0.1 & 0.3 & 0.3 & 0.2 & 0.10 & 0.10 & $\begin{array}{ll}0.1 & 0.0\end{array}$ & -0.3 & \\
\hline$Q_{f(10-70)}$ & 0.1 & -0.1 & -0.1 & -0.0 & 0.0 & 0.0 & 0.0 & 0.0 & 0.1 & 0.1 & 0.1 & 0.1 & 0.1 & 0.2 & 0.1 & 0.1 & 0.2 & 0.2 & 0.3 & 0.20 & $0.1-0$ & $\begin{array}{cc}-0.0 & -0.1\end{array}$ & -0.4 & -0.75 \\
\hline$f_{\text {urban }}(0.75-0.95)$ & 0.0 & 0.0 & 0.0 & 0.0 & 0.0 & 0.1 & 0.10 & 0.1 & 0.1 & 0.1 & 0.1 & 0.1 & 0.1 & 0.1 & 0.1 & 0.0 & -0.0 & -0.1 & 0.0 & $-0.0 \quad-c$ & $-0.0 \quad-0$ & $-0.0-0.0$ & -0.4 & \multirow{2}{*}{-0.50} \\
\hline$\lambda_{\text {wall }}(0.15-1.05)$ & -0.4 & -0.3 & -0.1 & -0.1 & -0.1 & -0.1 & $-0.1-c$ & $-0.1-$ & -0.0 & -0.1 & -0.0 & 0.0 & 0.0 & 0.0 & 0.0 & 0.0 & 0.1 & 0.1 & 0.1 & 0.10 & 0.00 & $\begin{array}{ll}0.0 & 0.0\end{array}$ & -0.2 & \\
\hline$C_{\text {wall }}(0.60-2.4)$ & -0.1 & -0.1 & -0.1 & -0.0 & -0.0 & -0.1 & $-0.1-0$ & -0.0 & -0.0 & -0.1 & -0.0 & 0.0 & -0.0 & 0.0 & 0.0 & 0.0 & 0.1 & 0.2 & 0.1 & 0.10 & 0.00 & $0.0 \quad 0.1$ & 0.5 & -0.25 \\
\hline$a_{\text {roof }}(0.10-0.30)$ & -0.2 & -0.1 & -0.0 & -0.1 & -0.1 & -0.1 & $-0.1-c$ & $-0.1-$ & -0.1 & -0.1 & -0.0 & -0.0 & -0.0 & 0.0 & 0.0 & 0.0 & 0.0 & 0.1 & 0.0 & 0.00 & 0.00 & $0.0 \quad 0.0$ & -0.1 & \multirow{2}{*}{-0.00} \\
\hline$A D V_{q}(0.67-1.5)$ & 0.0 & -0.0 & 0.0 & 0.0 & 0.0 & 0.0 & $0.0-c$ & -0.0 & -0.0 & 0.0 & -0.0 & -0.0 & 0.0 & 0.0 & 0.0 & 0.0 & 0.0 & 0.1 & 0.0 & 0.00 & 0.00 & $0.0 \quad 0.0$ & 0.1 & \\
\hline$A D V_{\Theta}(0.67-1.5)$ & -0.1 & -0.2 & -0.0 & 0.0 & -0.0 & $-0.0-c$ & $-0.3-6$ & -0.3 & -0.3 & -0.3 & -0.3 & -0.3 & -0.3 & -0.3 & -0.3 & -0.3 & -0.2 & -0.0 & 0.4 & 0.30 & 0.20 & $0.1 \quad 0.1$ & -0.1 & \multirow{4}{*}{$\begin{array}{l}-0.25 \\
-0.50 \\
-0.75\end{array}$} \\
\hline $\mathrm{CH}_{\text {mom }}(0.67-1.5)$ & 0.1 & 0.1 & 0.1 & 0.1 & 0.0 & 0.10 & 0.0 & 0.0 & 0.0 & 0.0 & -0.0 & -0.0 & -0.0 & -0.1 & -0.1 & -0.1 & -0.1 & -0.1 & -0.1 & -0.1 & $-0.1-0$ & $-0.0 \quad 0.0$ & -0.2 & \\
\hline $\mathrm{CH}_{\text {heat }(0.67-1.5)}$ & 0.2 & 0.1 & 0.1 & 0.1 & 0.1 & 0.10 & 0.10 & 0.00 & 0.0 & 0.0 & -0.0 & -0.0 & -0.1 & -0.1 & -0.1 & -0.1 & -0.2 & -0.3 & -0.2 & $-0.2-c$ & $-0.1-0$ & $-0.0 \quad 0.0$ & 0.3 & \\
\hline $\mathrm{CO}_{2}(38-3800)$ & -0.0 & -0.1 & -0.1 & -0.0 & -0.0 & 0.00 & 0.0 & $0.0-0$ & -0.0 & 0.1 & 0.0 & 0.0 & 0.0 & 0.0 & 0.0 & 0.0 & 0.1 & 0.1 & 0.1 & 0.10 & 0.10 & $0.0 \quad 0.1$ & 0.2 & \\
\hline \multirow[t]{2}{*}{$A O D_{(0.0-0.36)}$} & -0.2 & 0.1 & 0.1 & 0.0 & 0.0 & -0.0 & 0.0 & 0.00 & 0.0 & -0.0 & -0.0 & -0.0 & -0.0 & -0.0 & -0.0 & -0.0 & -0.0 & -0.0 & -0.0 & 0.0 & 0.00 & $\begin{array}{ll}0.0 & -0.0\end{array}$ & -0.3 & \multirow{2}{*}{-1.00} \\
\hline & 0 & & 2 & 4 & 4 & 0 & & 8 & 3 & & & 12 & 12 & 14 & & 16 & 0 & 18 & & 20 & & 22 & 24 & \\
\hline
\end{tabular}

(b)

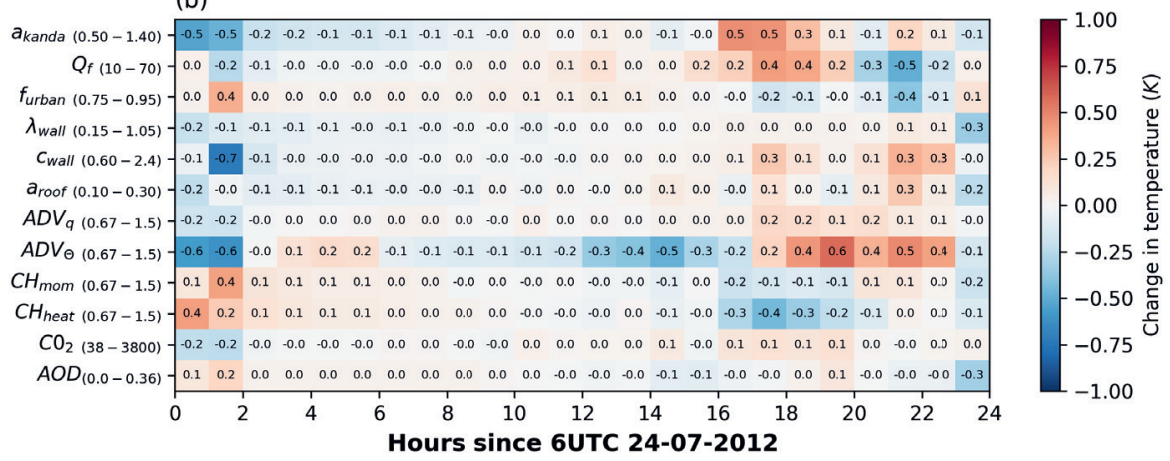

Figure A.3.9: As Figure A.3.7, but for the hourly change in $50 \mathrm{~m}$ temperature $\left(T_{50 \mathrm{~m}}\right)$ 
(a)

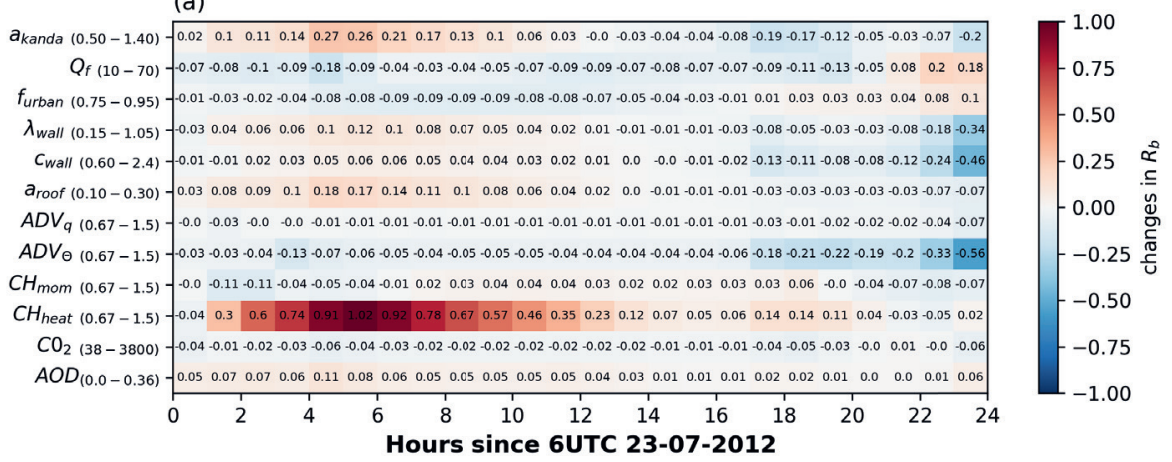

(b)

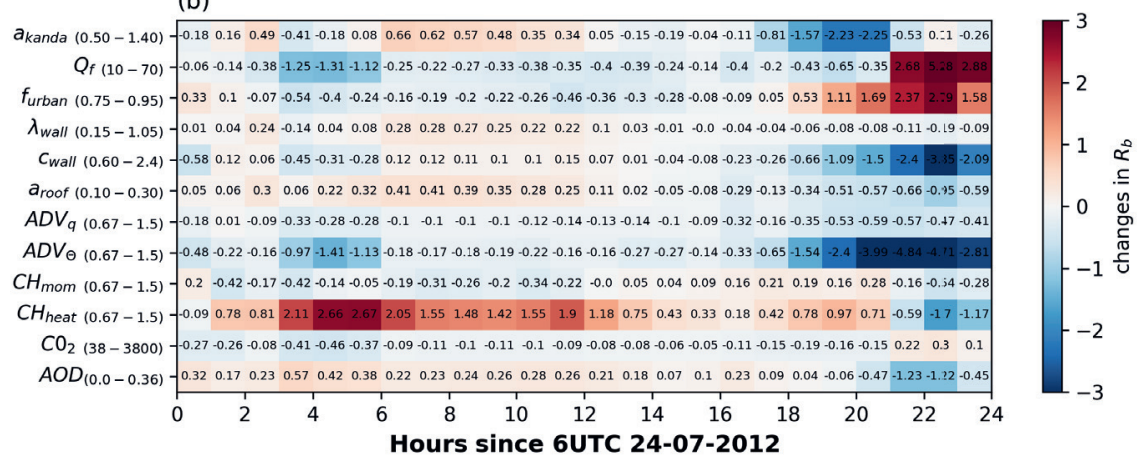

Figure A.3.10: As Figure A.3.7, but for the bulk Richardson number $\left(R i_{b}\right)$ 


\section{A.4 Chapter 4: Supplementary material}

This section contains the supplementary figures A.4.1-4.4

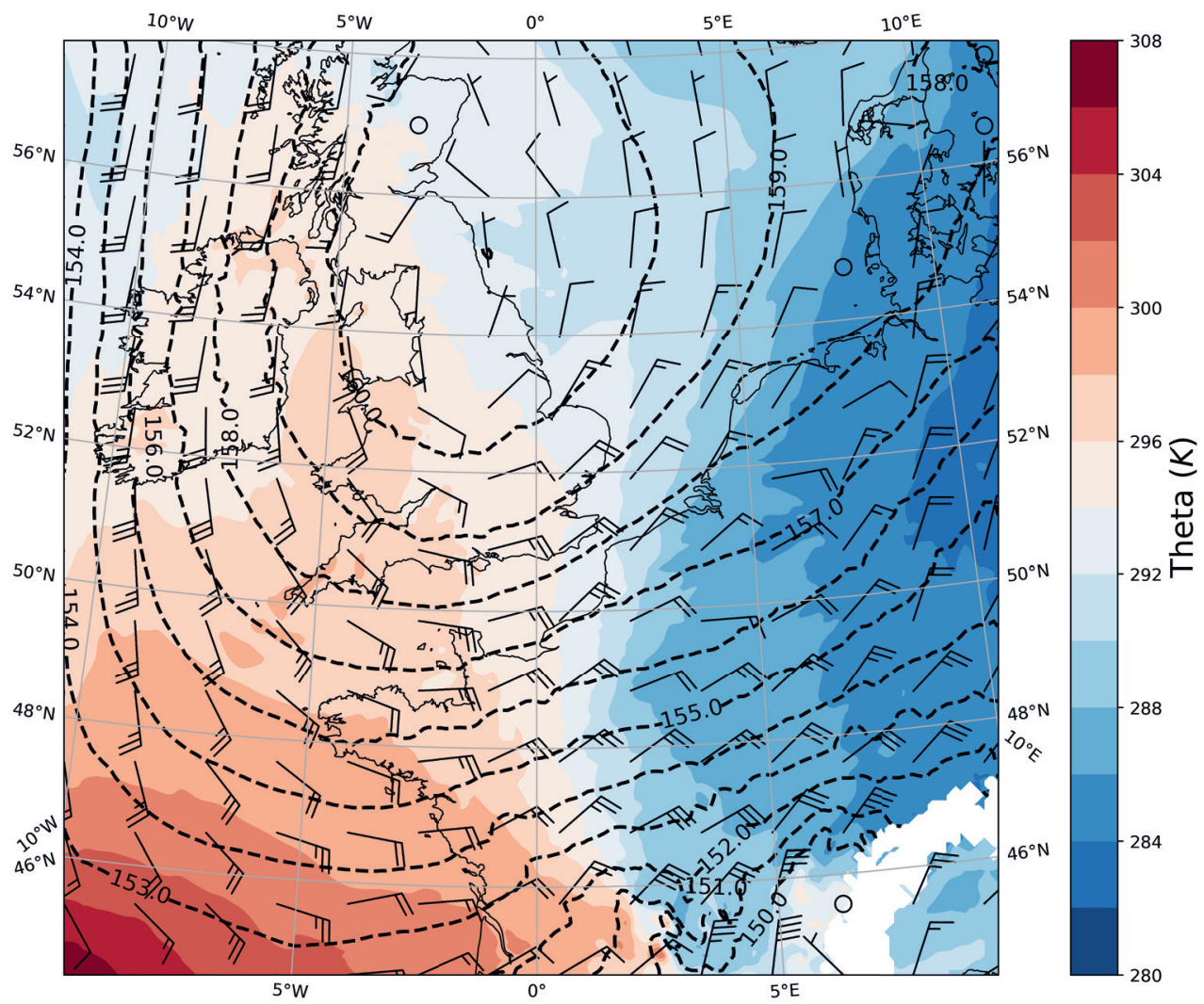

Figure A.4.1: Modelled geopotential height (dm, dotted contours), potential temperature (K, filled contours) and wind barbs (in kts) with the WRF model (outer domain) at $850 \mathrm{hPa}$ during the first night (at 2100 UTC 14/05/2019). 


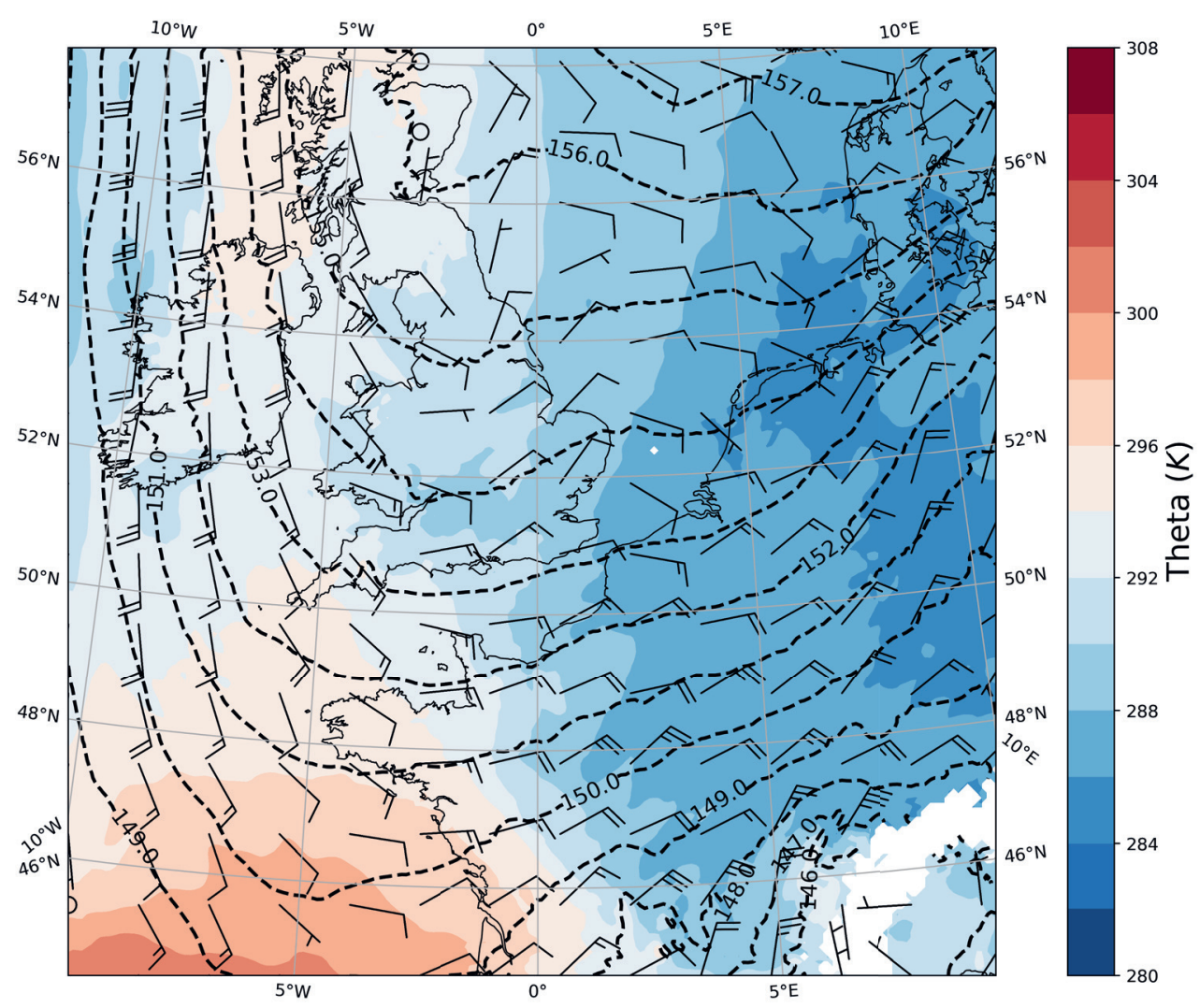

Figure A.4.2: Modelled geopotential height (dm, dotted contours), potential temperature (K, filled contours) and wind barbs (in kts) with the WRF model (outer domain) at 850hPa during the second night (at 2100 UTC 15/05/2019). 


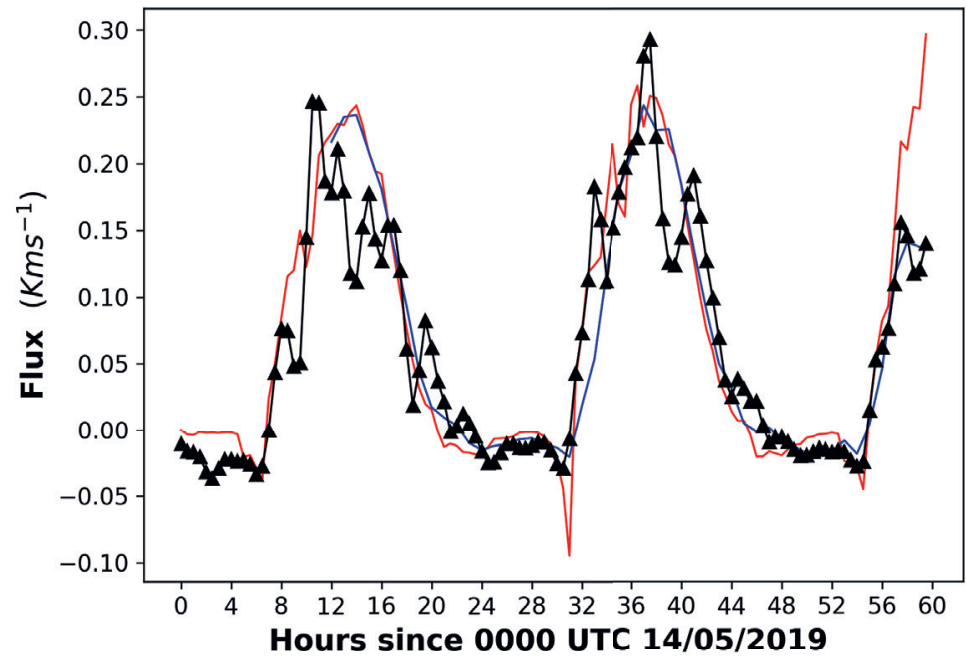

- WRF UKV $\simeq$ Observations

Figure A.4.3: Kinematic heat flux (K m ) at $175 \mathrm{~m}$ a.g.l over the BT tower, London from the WRF (blue line) and UKV (red) models and observations (black triangles) during the case study period (0000 UTC 14/05/2019 to 1200UTC 16/05/2019). 

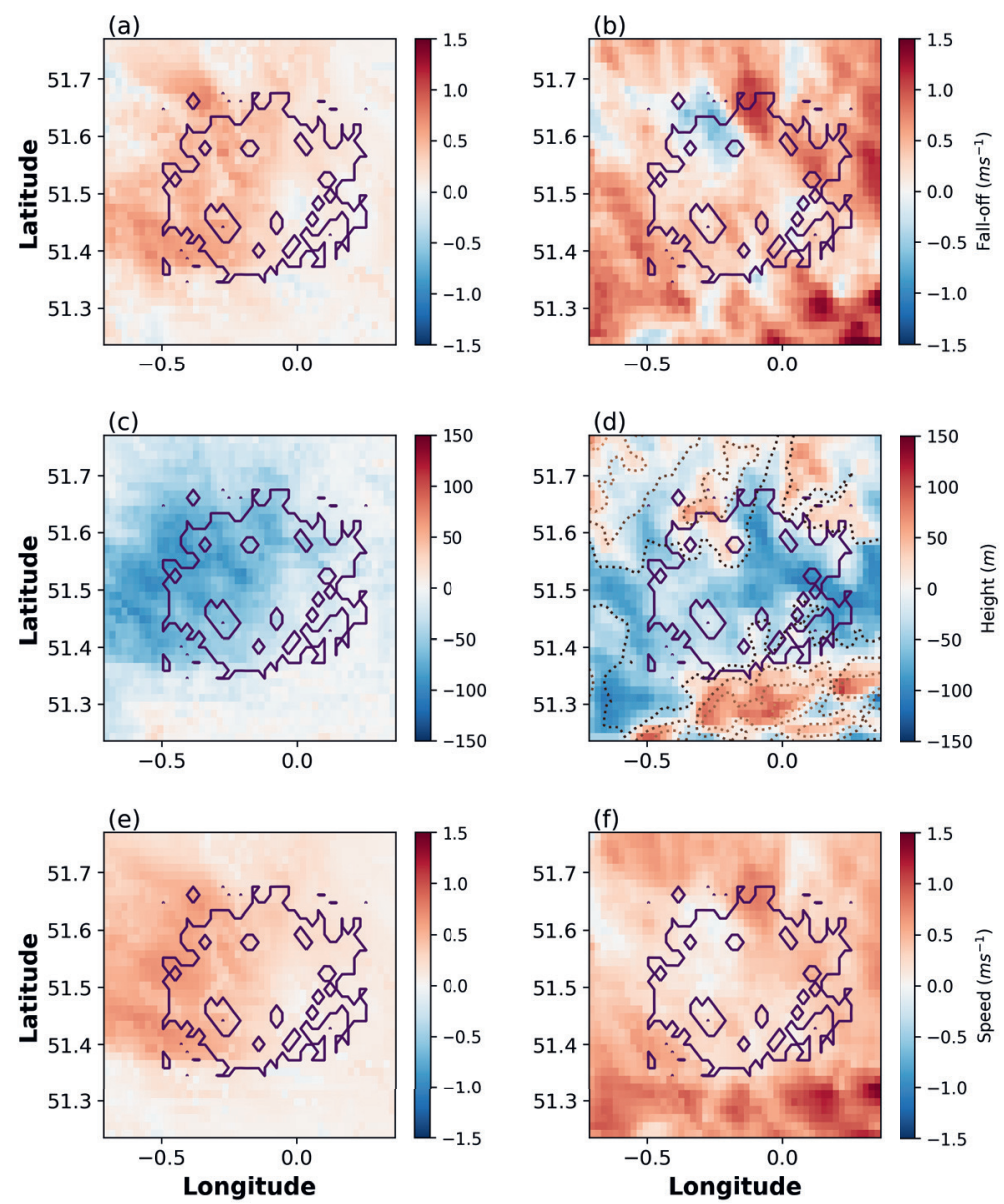

Figure A.4.4: As Figure 4.6.2, but for the second night (2100 UTC 15/05/2019 to 0600 UTC 16/05/2019) 


\section{A.5 Chapter 5: Supplementary material}

This section contains supplementary figures with the initial forcing conditions for the 1D-WRF and PALM models.
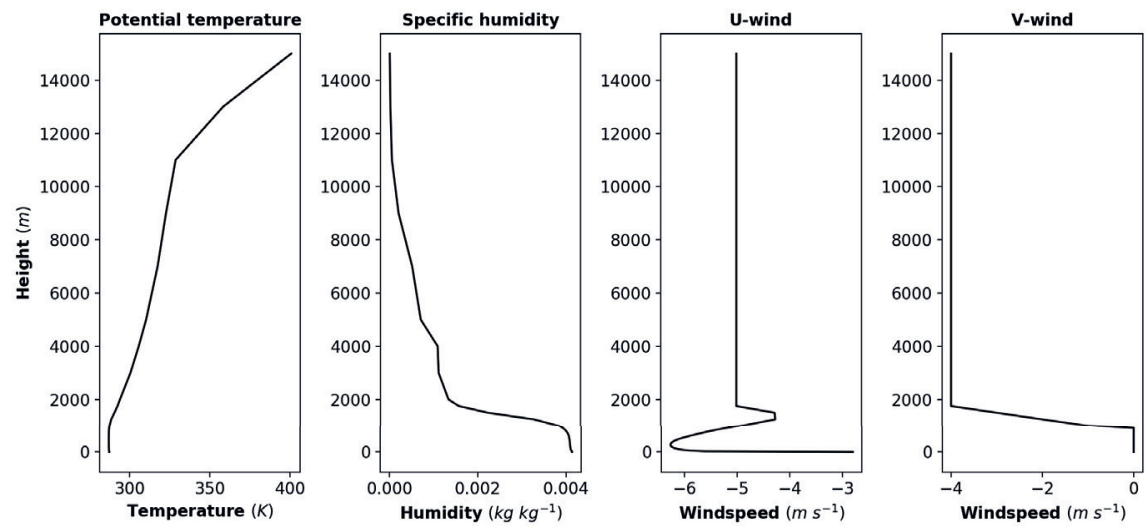

Figure A.5.1: Initial forcing profiles of potential temperature $(K)$, specific humidity $\left(k g \mathrm{~kg}^{-1}\right), \mathrm{u}$ and $\mathrm{v}$ wind components $\left(\mathrm{m} \mathrm{s}^{-1}\right)$ for the 1D-WRF model. These profiles are taken from a 3D-WRF simulation at 1700 UTC May 2019 and are an average of profiles in an $30 \mathrm{x} 30 \mathrm{~km}$ area over London. Initial v wind component is set to $0 \mathrm{~m} \mathrm{~s}^{-1}$ in the lowest $1250 \mathrm{~m}$ to match the forcing profiles in the PALM model.
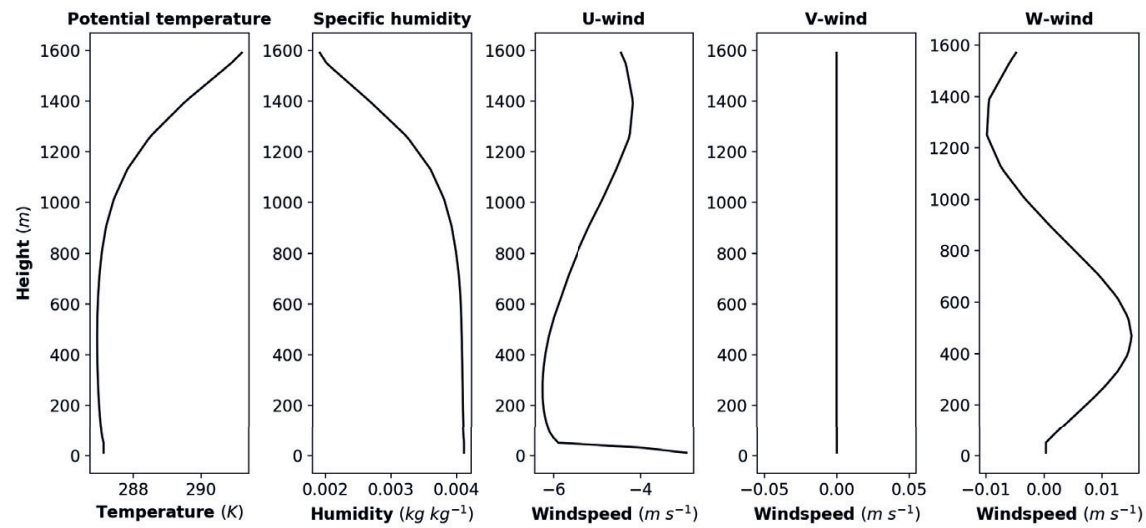

Figure A.5.2: Initial forcing profiles of potential temperature $(K)$, specific humidity $\left(k g \mathrm{~kg}^{-1}\right), \mathrm{u}, \mathrm{v}$ and $\mathrm{w}$ wind components $\left(m s^{-1}\right)$ for the PALM model. These profiles are taken from a 3D-WRF simulation at 1700 UTC May 2019 and are an average of profiles in an $30 \mathrm{x} 30 \mathrm{~km}$ area over London. Initial $\mathrm{v}$ wind component is set to $0 \mathrm{~m} \mathrm{~s}^{-1}$ in the forcing profile of the PALM model. 

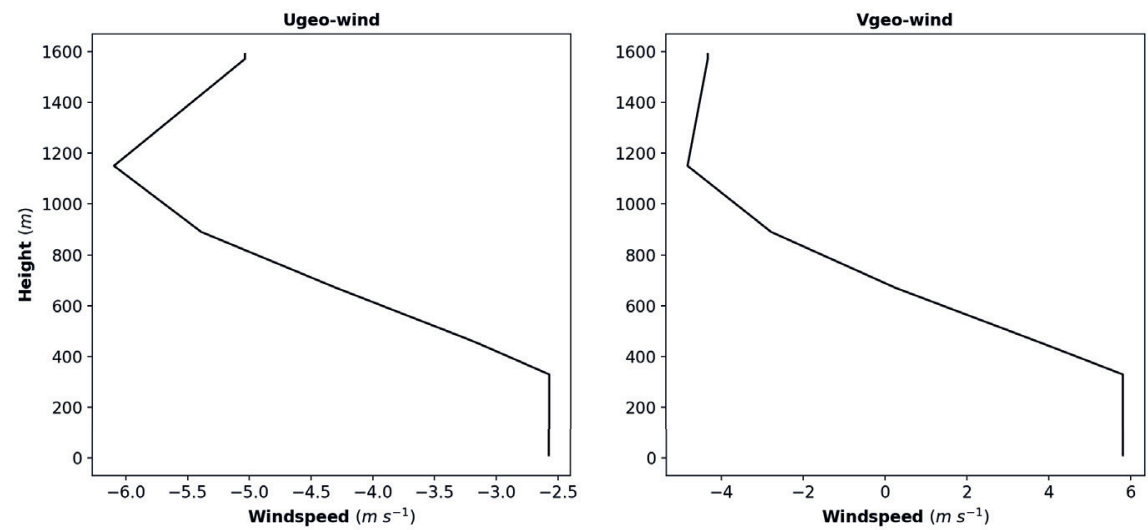

Figure A.5.3: Forcing profiles of $u$ and $v$ geostrophic wind speed $\left(\mathrm{m} \mathrm{s}^{-1}\right)$ for the 1D-WRF and PALM model. These profiles are calculated from the geo-potential at isobaric levels in a 3D-WRF simulation at 1700 UTC May 2019 and are an average over an area of $45 \times 45 \mathrm{~km}$ over London to ensure consistency.
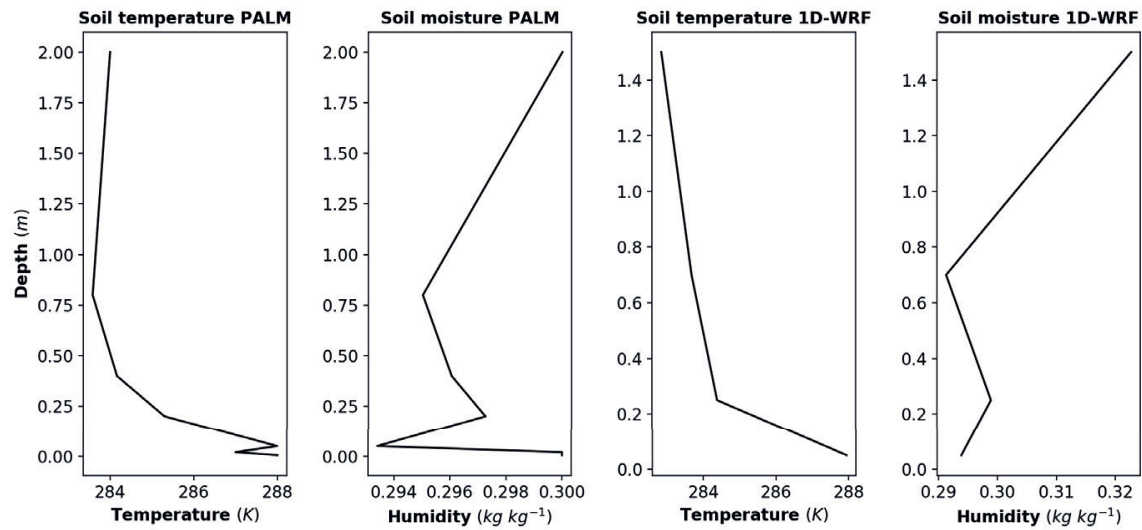

Figure A.5.4: Initial forcing profiles for soil temperature $(K)$ and moisture $\left(\mathrm{kg} \mathrm{kg}^{-1}\right)$ for the 1D-WRF and PALM models. These soil profiles are taken from a 3D-WRF simulation at 1700 UTC May 2019 over London. 

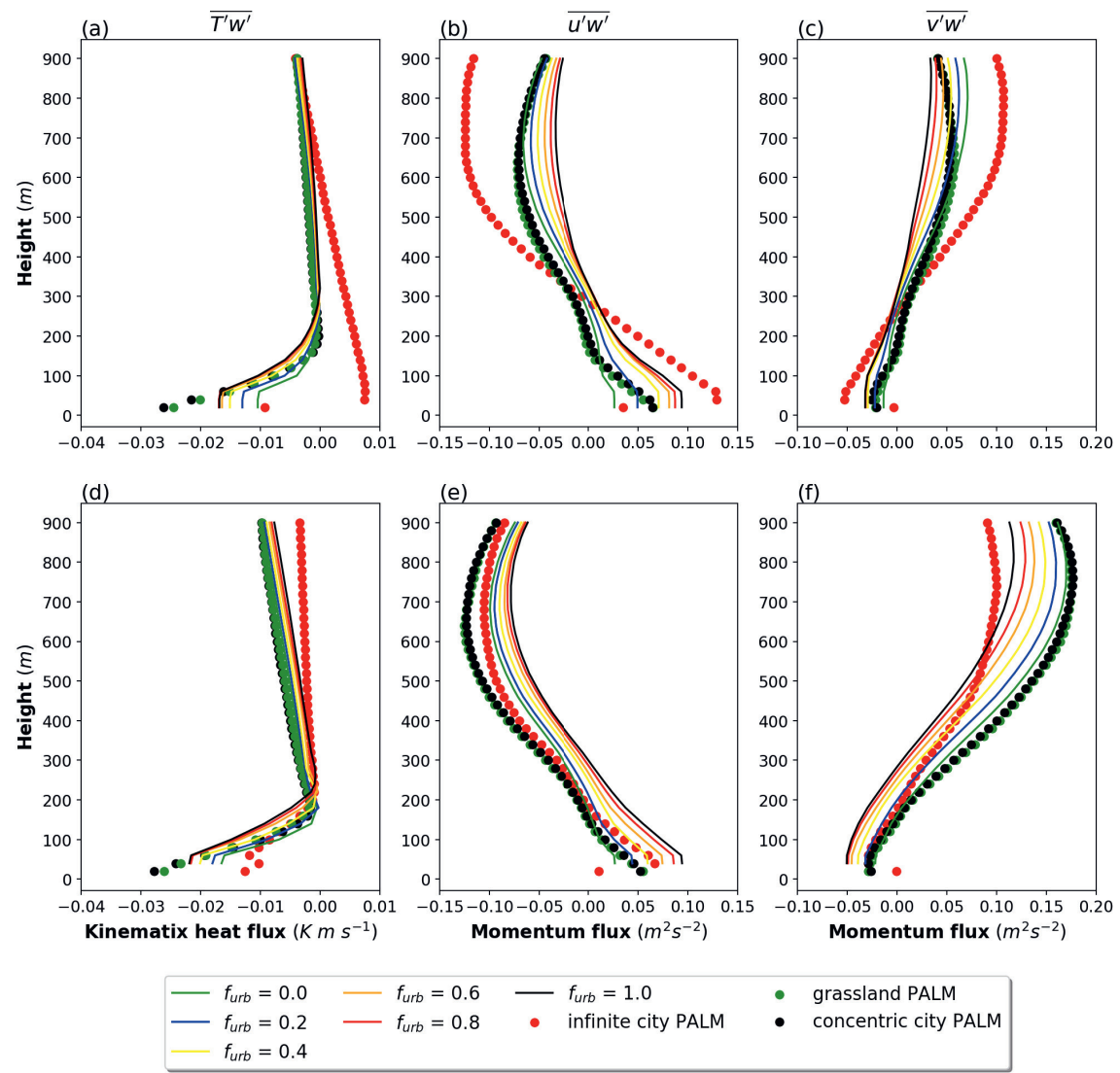

- grassland PALM

- concentric city PALM

Figure A.5.5: Vertical profiles over (a,d) the modelled vertical kinematic heat $\left(K \mathrm{~m} \mathrm{~s}^{-1}\right)$, $(\mathrm{b}, \mathrm{e}) \mathrm{u}$ and $(\mathrm{c}, \mathrm{f}) \mathrm{v}$ momentum $\left(m^{2} s^{-2}\right)$ fluxes (parameterized + resolved) from the 1D-WRF (lines) and PALM (circles) models. The profiles are taken at $\mathrm{t}=8400 \mathrm{~s}(\mathrm{a}, \mathrm{b}, \mathrm{c})$ and $\mathrm{t}=$ $14400 \mathrm{~s}(\mathrm{c}, \mathrm{d}, \mathrm{e})$ after the initialisation of the model at 1700 UTC 15 May 2019. Experiments with different urban fractions $\left(f_{u r b}\right)$ are depicted with different colours (see legend) as are the PALM grassland (green), infinite city (red) and concentric city (black) runs. 


\section{References}

Arakawa, H. (1937). Increasing air temperatures in large developing cities. Beitr. Geophys., 50, 3-6.

Arnfield, A. J. (2003). Two decades of urban climate research: a review of turbulence, exchanges of energy and water, and the urban heat island. International Journal of Climatology, 23, 1-26. doi:doi: 10.1002/joc.859.

Baas, P., Bosveld, F. C., Klein Baltink, H., \& Holtslag, A. A. M. (2009). A climatology of nocturnal low-level jets at cabauw. Journal of Applied Meteorology and Climatology, 48, 1627-1642. doi:doi: 10.1175/2009JAMC1965.1.

Baklanov, A., Molina, L. T., \& Gauss, M. (2016). Megacities, air quality and climate. Atmospheric Environment, 126, 235 - 249. doi:doi: https://doi.org/10.1016/j.atmosenv.2015.11.059.

Balsamo, G., Beljaars, A., Scipal, K., Viterbo, P., van den Hurk, B., Hirschi, M., \& Betts, A. K. (2009). A Revised Hydrology for the ECMWF Model: Verification from Field Site to Terrestrial Water Storage and Impact in the Integrated Forecast System. Journal of Hydrometeorology, 10, 623-643. doi:doi: 10.1175/2008JHM1068.1.

Banakh, V., \& Smalikho, I. (2018). Lidar studies of wind turbulence in the stable atmospheric boundary layer. Remote Sensing, 10. doi:doi: 10.3390/rs10081219.

Banta, R. M., Darby, L. S., Fast, J. D., Pinto, J. O., Whiteman, C. D., Shaw, W. J., \& Orr, B. W. (2004). Nocturnal Low-Level Jet in a Mountain Basin Complex. Part I: Evolution and Effects on Local Flows. Journal of Applied Meteorology, 43, 1348-1365. doi:doi: 10.1175/JAM2142.1.

Banta, R. M., Pichugina, Y. L., \& Brewer, W. A. (2006). Turbulent velocity-variance profiles in the stable boundary layer generated by a nocturnal low-level jet. Journal of the Atmospheric Sciences, 63, 2700-2719. doi:doi: 10.1175/JAS3776.1.

Banta, R. M., Pichugina, Y. L., \& Newsom, R. K. (2003). Relationship between lowlevel jet properties and turbulence kinetic energy in the nocturnal stable boundary layer. Journal of the Atmospheric Sciences, 60, 2549-2555. doi:doi: 10.1175/15200469(2003)060¡2549:RBLJPA¿2.0.CO;2.

Bao, J.-W., Michelson, S. A., Persson, P. O. G., Djalalova, I. V., \& Wilczak, J. M. (2008). 
Observed and WRF-Simulated Low-Level Winds in a High-Ozone Episode during the Central California Ozone Study. Journal of Applied Meteorology and Climatology, 47, 2372-2394. doi:doi: 10.1175/2008JAMC1822.1.

Barlow, J. F. (2014). Progress in observing and modelling the urban boundary layer. Urban Climate, 10, 216 - 240. doi:doi: https://doi.org/10.1016/j.uclim.2014.03.011.

Barlow, J. F., Halios, C. H., Lane, S. E., \& Wood, C. R. (2015). Observations of urban boundary layer structure during a strong urban heat island event. Environmental Fluid Mechanics, 15, 373 - 398. doi:doi: 10.1007/s10652-014-9335-6.

Bassett, R., Cai, X., Chapman, L., Heaviside, C., \& Thorne, J. (2017). The effects of heat advection on uk weather and climate observations in the vicinity of small urbanized areas. Boundary-Layer Meteorology, 165, 181-196. doi:doi: 10.1007/s10546-017-0263-0.

Bassett, R., Cai, X., Chapman, L., Heaviside, C., \& Thornes, J. E. (2019). Semiidealized urban heat advection simulations using the weather research and forecasting mesoscale model. International Journal of Climatology, 39, 1345-1358. doi:doi: https://doi.org/10.1002/joc.5885.

Best, M., Pryor, M., Clark, D., Rooney, G., Essery, R., Ménard, C., Edwards, J., Hendry, M., Porson, A., Gedney, N. et al. (2011). The joint uk land environment simulator (jules), model description-part 1: energy and water fluxes. Geoscientific Model Development, 4, 677-699.

Best, M. J. (2005). Representing urban areas within operational numerical weather prediction models. Boundary-Layer Meteorology, 114, 91-109. doi:doi: 10.1007/s10546004-4834-5.

Best, M. J., \& Grimmond, C. S. B. (2015). Key Conclusions of the First International Urban Land Surface Model Comparison Project. Bulletin of the American Meteorological Society, 96, 805-819. doi:doi: 10.1175/BAMS-D-14-00122.1.

Best, M. J., \& Grimmond, C. S. B. (2016). Investigation of the impact of anthropogenic heat flux within an urban land surface model and pilps-urban. Theoretical and Applied Climatology, 126, 51-60. doi:doi: 10.1007/s00704-015-1554-3.

Blackadar, A. K. (1957). Boundary layer wind maxima and their significance for the growth of nocturnal inversions. Bulletin of the American Meteorological Society, 38, 283-290. doi:doi: 10.1175/1520-0477-38.5.283.

Bohnenstengel, S., Evans, S., Clark, P., \& Belcher, S. (2011). Simulations of the London urban heat island. Quarterly Journal of the Royal Meteorological Society, 137, 16251640. doi:doi: 10.1002/qj.855.

Bohnenstengel, S., Hamilton, I., Davies, M., \& Belcher, S. (2014). Impact of anthropogenic heat emissions on london's temperatures. Quarterly Journal of the Royal Meteorological Society, 140, 687-698. doi:doi: 10.1002/qj.2144. 
Bornstein, R. (1975). The two-dimensional urbmet urban boundary layer model. Journal of Applied Meteorology, 14, 1459-1477. doi:doi: 10.1175/15200450(1975)014¡1459:ttduub¿2.0.co;2.

Bornstein, R., \& Johnson, D. (1977). Urban-rural wind velocity differences. Atmospheric Environment (1967), 11, 597-604. doi:doi: 10.1016/0004-6981(77)90112-3.

Bornstein, R., \& Lin, Q. (2000). Urban heat islands and summertime convective thunderstorms in atlanta: three case studies. Atmospheric Environment, 34, 507 - 516. doi:doi: https://doi.org/10.1016/S1352-2310(99)00374-X.

Bosveld, F. C., Baas, P., van Meijgaard, E., de Bruijn, E. I. F., Steeneveld, G.-J., \& Holtslag, A. A. M. (2014a). The third gabls intercomparison case for evaluation studies of boundary-layer models. part a: Case selection and set-up. Boundary-Layer Meteorology, 152, 133-56. doi:doi: 10.1371/journal.pone.0214474.

Bosveld, F. C. et al. (2014b). The third gabls intercomparison case for evaluation studies of boundary-layer models. part b: Results and process understanding. Boundary-Layer Meteorology, 152, 157-187. doi:doi: 10.1007/s10546-014-9919-1.

Boutle, I., Eyre, J., \& Lock, A. (2014). Seamless stratocumulus simulation across the turbulent gray zone. Monthly Weather Review, 142, 1655-1668.

Buccolieri, R., Santiago, J., \& Martilli, A. (). Cfd modelling: The most useful tool for developing mesoscale urban canopy parameterizations. Building Simulation, . doi:doi: 10.1007/s12273-020-0689-Z.

Büdel, H., \& Wolf, J. (1933). Münchener stadtklimatische studien. Wetter, 49, 4-10.

Cadenasso, M. L., Pickett, S. T. A., \& Schwarz, K. (2007). Spatial heterogeneity in urban ecosystems: reconceptualizing land cover and a framework for classification. Frontiers in Ecology and the Environment, 5, 80-88. doi:doi: 10.1890/15409295(2007)5[80:SHIUER]2.0.CO;2.

Chandler, T. (1964). City growth and urban climates. Weather, 19, 170-171. doi:doi: 10.1002/j.1477-8696.1964.tb02116.x.

Chandler, T. (1967). Night-time temperatures in relation to leicester's urban form. Meteorol. Mag., 96, 244-250.

Changnon, S. A., Huff, F. A., \& Semonin, R. G. (1971). Metromex: an investigation of inadvertent weather modification. Bulletin of the American Meteorological Society, 52, 958-967.

Chen, F., Bornstein, R., Grimmond, S., Li, J., Liang, X., Martilli, A., Miao, S., Voogt, J., \& Wang, Y. (2012). Research Priorities in Observing and Modeling Urban Weather and Climate. Bulletin of the American Meteorological Society, 93, 1725-1728. doi:doi: 10.1175/BAMS-D-11-00217.1.

Chen, F., \& Dudhia, J. (2001). Coupling an Advanced Land Surface-Hydrology Model 
with the Penn State-NCAR MM5 Modeling System. Part I: Model Implementation and Sensitivity. Monthly Weather Review, 129, 569-585. doi:doi: 10.1175/15200493(2001)129;0569:CAALSH ¿2.0.CO;2.

Chen, F. et al. (2011a). The integrated WRF/urban modelling system: Development, evaluation, and applications to urban environmental problems. International Journal of Climatology, 31, 273-288. doi:doi: 10.1002/joc.2158.

Chen, F., Miao, S., Tewari, M., Bao, J.-W., \& Kusaka, H. (2011b). A numerical study of interactions between surface forcing and sea breeze circulations and their effects on stagnation in the greater houston area. Journal of Geophysical Research: Atmospheres, 116. doi:doi: 10.1029/2010JD015533.

Ching, J. et al. (2018). World urban database and access portal tools (wudapt), an urban weather, climate and environmental modeling infrastructure for the anthropocene. Bulletin of the American Meteorological Society, 0, null. doi:doi: 10.1175/BAMS-D-160236.1.

Clough, S., Shephard, M., Mlawer, E., Delamere, J., Iacono, M., Cady-Pereira, K., Boukabara, S., \& Brown, P. (2005). Atmospheric radiative transfer modeling: a summary of the aer codes. Journal of Quantitative Spectroscopy and Radiative Transfer, 91, 233 - 244. doi:doi: https://doi.org/10.1016/j.jqsrt.2004.05.058.

Coceal, O., Bohnenstengel, S. I., \& Kotthaus, S. (2018). Detection of sea-breeze events around london using a fuzzy-logic algorithm. Atmospheric Science Letters, 19, e846. doi:doi: 10.1002/asl.846.

Collins, W., Rasch, P., \& Coauthors (2004). Description of the ncar community atmosphere model (cam 3.0). ncar tech. note ncar/tn-464+str. 214 pp.

Demuzere, M., Bechtel, B., Middel, A., \& Mills, G. (2019). Mapping europe into local climate zones. PLOS ONE, 14, 1-27. doi:doi: 10.1371/journal.pone.0214474.

Demuzere, M., Harshan, S., Järvi, L., Roth, M., Grimmond, C. S. B., Masson, V., Oleson, K. W., Velasco, E., \& Wouters, H. (2017). Impact of urban canopy models and external parameters on the modelled urban energy balance in a tropical city. Quarterly Journal of the Royal Meteorological Society, 143, 1581-1596. doi:doi: 10.1002/qj.3028.

Dong, Y., Varquez, A., \& Kanda, M. (2017). Global anthropogenic heat flux database with high spatial resolution. Atmospheric Environment, 150, 276 - 294. doi:doi: https://doi.org/10.1016/j.atmosenv.2016.11.040.

Droste, A. M., Steeneveld, G. J., \& Holtslag, A. A. M. (2018). Introducing the urban wind island effect. Environmental Research Letters, 13, 094007. doi:doi: 10.1088/17489326/aad8ef.

Dudhia, J. (1989). Numerical Study of Convection Observed during the Winter Monsoon Experiment Using a Mesoscale Two-Dimensional Model. Jour- 
nal of the Atmospheric Sciences, 46, 3077-3107. doi:doi: 10.1175/15200469(1989)046¡3077:NSOCOD ¿2.0.CO;2.

ECMWF (2012). Atmospheric Model high resolution 10-day forecast (HRES).

Emeis, S. (2014). Current issues in wind energy meteorology. Meteorological Applications, 21, 803-819. doi:doi: 10.1002/met.1472.

Engineering Toolbox (2010). Thermal conductivity of some common materials [online] Available at: https://www.engineeringtoolbox.com [Accessed 13-05-2017], .

Ferrero, E., Alessandrini, S., \& Vandenberghe, F. (2018). Assessment of PlanetaryBoundary-Layer Schemes in the Weather Research and Forecasting Model Within and Above an Urban Canopy Layer. Boundary-Layer Meteorology, 168, 289-319. doi:doi: 10.1007/s10546-018-0349-3.

Flagg, D. D., \& Taylor, P. A. (2011). Sensitivity of mesoscale model urban boundary layer meteorology to the scale of urban representation. Atmospheric Chemistry and Physics, 11, 2951-2972. doi:doi: 10.5194/acp-11-2951-2011.

Gentine, P., Steeneveld, G.-J., Heusinkveld, B. G., \& Holtslag, A. A. (2018). Coupling between radiative flux divergence and turbulence near the surface. Quarterly Journal of the Royal Meteorological Society, 144, 2491-2507. doi:doi: https://doi.org/10.1002/qj.3333.

Gomes, L., Mallet, M., Roger, J. C., \& Dubuisson, P. (2008). Effects of the physical and optical properties of urban aerosols measured during the capitoul summer campaign on the local direct radiative forcing. Meteorology and Atmospheric Physics, 102, 289. doi:doi: 10.1007/s00703-008-0321-8.

Grimmond, C. S. B. et al. (2011). Initial results from Phase 2 of the international urban energy balance model comparison. International Journal of Climatology, 31, 244-272. doi:doi: $10.1002 /$ joc. 2227 .

Grimmond, C. S. B. et al. (2010). The international urban energy balance models comparison project: First results from phase 1. Journal of Applied Meteorology and Climatology, 49, 1268-1292. doi:doi: 10.1175/2010JAMC2354.1.

Grimmond, C. S. B., \& Oke, T. R. (1991). An evapotranspiration-interception model for urban areas. Water Resources Research, 27, 1739-1755. doi:doi: 10.1029/91WR00557.

Grimmond, C. S. B., \& Oke, T. R. (1999). Heat Storage in Urban Areas: Local-Scale Observations and Evaluation of a Simple Model. Journal of Applied Meteorology, 38, 922-940. doi:doi: 10.1175/1520-0450(1999)038¡0922:HSIUAL¿2.0.CO;2.

Grimmond, C. S. B., Salmond, J. A., Oke, T. R., Offerle, B., \& Lemonsu, A. (2004). Flux and turbulence measurements at a densely built-up site in marseille: Heat, mass (water and carbon dioxide), and momentum. Journal of Geophysical Research: Atmospheres, 109. doi:doi: 10.1029/2004JD004936. 
Halios, C. H., \& Barlow, J. F. (2018). Observations of the morning development of the urban boundary layer over london, uk, taken during the actual project. Boundary-Layer Meteorology, 166, 395-422. doi:doi: 10.1007/s10546-017-0300-z.

Harman, I. N., Barlow, J. F., \& Belcher, S. E. (2004). Scalar fluxes from urban street canyons part ii: model. Boundary-Layer Meteorology, 113, 387-410.

Heaviside, C., Cai, X.-M., \& Vardoulakis, S. (2015). The effects of horizontal advection on the urban heat island in birmingham and the west midlands, united kingdom during a heatwave. Quarterly Journal of the Royal Meteorological Society, 141, 1429-1441. doi:doi: 10.1002/qj.2452.

van Heerwaarden, C. C., Vilà-Guerau de Arellano, J., Moene, A. F., \& Holtslag, A. A. M. (2009). Interactions between dry-air entrainment, surface evaporation and convective boundary-layer development. Quarterly Journal of the Royal Meteorological Society, 135, 1277-1291. doi:doi: 10.1002/qj.431.

Heus, T., van Heerwaarden, C. C., Jonker, H. J. J., Pier Siebesma, A., Axelsen, S., van den Dries, K., Geoffroy, O., Moene, A. F., Pino, D., de Roode, S. R., \& Vilà-Guerau de Arellano, J. (2010). Formulation of the dutch atmospheric large-eddy simulation (dales) and overview of its applications. Geoscientific Model Development, 3, 415-444. doi:doi: 10.5194/gmd-3-415-2010.

Holton, J. R. (1967). The diurnal boundary layer wind oscillation above sloping terrain. Tellus, 19, 200-205. doi:doi: 10.3402/tellusa.v19i2.9766.

Hong, S.-Y., Dudhia, J., \& Chen, S.-H. (2004). A Revised Approach to Ice Microphysical Processes for the Bulk Parameterization of Clouds and Precipitation. Monthly Weather Review, 132, 103-120. doi:doi: 10.1175/1520-0493(2004)132¡0103:ARATIM ¿2.0.CO;2.

Hong, S.-Y., Noh, Y., \& Dudhia, J. (2006). A New Vertical Diffusion Package with an Explicit Treatment of Entrainment Processes. Monthly Weather Review, 134, 23182341. doi:doi: 10.1175/MWR3199.1.

Howard, L. (2012). The Climate of London: Deduced from Meteorological Observations (1833) volume 1 of Cambridge Library Collection - Earth Science. Cambridge University Press. doi:doi: 10.1017/CBO9781139226899.

Hu, X.-M., Klein, P., Xue, M., Lundquist, J., Zhang, F., \& Qi, Y. (2013a). Impact of low-level jets on the nocturnal urban heat island intensity in oklahoma city. Journal of Applied Meteorology and Climatology, 52, 1779-1802. doi:doi: 10.1175/JAMC-D-120256.1.

Hu, X.-M., Klein, P. M., Xue, M., Lundquist, J. K., Zhang, F., \& Qi, Y. (2013b). Impact of Low-Level Jets on the Nocturnal Urban Heat Island Intensity in Oklahoma City. Journal of Applied Meteorology and Climatology, 52, 1779-1802. doi:doi: 10.1175/JAMCD-12-0256.1. 
Hutcheon, R., Johnson, R., Lowry, W., Black, C., \& Hadley, D. (1967). Observations of the heat island in a small city. Bulletin of the American Meteorological Society, 48 , $7-9$.

Iacono, M. J., Delamere, J. S., Mlawer, E. J., Shephard, M. W., Clough, S. A., \& Collins, W. D. (2008). Radiative forcing by long-lived greenhouse gases: Calculations with the AER radiative transfer models. Journal of Geophysical Research: Atmospheres, 113. doi:doi: 10.1029/2008JD009944.

Iamarino, M., Beevers, S., \& Grimmond, C. S. B. (2012). High-resolution (space, time) anthropogenic heat emissions: London 1970-2025. International Journal of Climatology, 32, 1754-1767. doi:doi: 10.1002/joc.2390.

Janjic, Z. I. (1994). The Step-Mountain Eta Coordinate Model: Further Developments of the Convection, Viscous Sublayer, and Turbulence Closure Schemes. Monthly Weather Review, 122, 927-945. doi:doi: 10.1175/1520-0493(1994)122¡0927:TSMECM ¿2.0.CO;2.

Jarvi, L., Grimmond, C., \& Christen, A. (2011). The Surface Urban Energy and Water Balance Scheme (SUEWS): Evaluation in Los Angeles and Vancouver. Journal of Hydrology, 411, 219-237. doi:doi: https://doi.org/10.1016/j.jhydrol.2011.10.001.

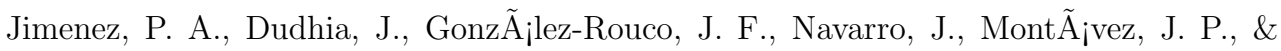
GarcÃa-Bustamante, E. (2012). A revised scheme for the wrf surface layer formulation. Monthly Weather Review, 140, 898-918. doi:doi: 10.1175/MWR-D-11-00056.1.

Kallistratova, M. A., \& Kouznetsov, R. D. (2012). Low-level jets in the moscow region in summer and winter observed with a sodar network. Boundary-Layer Meteorology, 143, 159 - 175. doi:doi: 10.1007/s10546-011-9639-8.

Kalverla, P., Steeneveld, G.-J., Ronda, R., \& Holtslag, A. A. (2019a). Evaluation of three mainstream numerical weather prediction models with observations from meteorological mast ijmuiden at the north sea. Wind Energy, 22, 34-48. doi:doi: 10.1002/we.2267.

Kalverla, P. C., Duncan Jr., J. B., Steeneveld, G.-J., \& Holtslag, A. A. M. (2019b). Lowlevel jets over the north sea based on era5 and observations: together they do better. Wind Energy Science, 4, 193-209. doi:doi: 10.5194/wes-4-193-2019.

Kalverla, P. C., Steeneveld, G.-J., Ronda, R. J., \& Holtslag, A. A. (2017). An observational climatology of anomalous wind events at offshore meteomast ijmuiden (north sea). Journal of Wind Engineering and Industrial Aerodynamics, 165, 86 - 99. doi:doi: https://doi.org/10.1016/j.jweia.2017.03.008.

Kanda, M., Kanega, M., Kawai, T., Moriwaki, R., \& Sugawara, H. (2007). Roughness lengths for momentum and heat derived from outdoor urban scale models. Journal of Applied Meteorology and Climatology, 46, 1067-1079. doi:doi: 10.1175/JAM2500.1.

Katzfey, J., Schlünzen, H., Hoffmann, P., \& Thatcher, M. (2020). How an urban parameterization affects a high-resolution global climate simulation. Quar- 
terly Journal of the Royal Meteorological Society, 146, 3808-3829. doi:doi: https://doi.org/10.1002/qj.3874.

Kent, C. W., Lee, K., Ward, H. C., Hong, J.-W., Hong, J., Gatey, D., \& Grimmond, S. (2018). Aerodynamic roughness variation with vegetation: analysis in a suburban neighbourhood and a city park. Urban Ecosystems, 1, 227-243. doi:doi: 10.1007/s11252-0170710-1.

Kleczek, M. A., Steeneveld, G.-J., \& Holtslag, A. A. M. (2014). Evaluation of the Weather Research and Forecasting Mesoscale Model for GABLS3: Impact of Boundary-Layer Schemes, Boundary Conditions and Spin-Up. Boundary-Layer Meteorology, 152, 213243. doi:doi: 10.1007/s10546-014-9925-3.

Klein, P., Hu, X., Shapiro, A., \& Xue, M. (2016). Linkages Between Boundary-Layer Structure and the Development of Nocturnal Low-Level Jets in Central Oklahoma. Boundary-Layer Meteorology, 158, 383-408. doi:doi: 10.1007/s10546-015-0097-6.

Klein, P., Hu, X., \& Xue, M. (2014). Impacts of mixing processes in nocturnal atmospheric boundary layer on urban ozone concentrations. Boundary-Layer Meteorology, 150, 107130. doi:doi: 10.1007/s10546-013-9864-4.

Kokkonen, T. V., Grimmond, S., Murto, S., Liu, H., Sundström, A.-M., \& Järvi, L. (2019). Simulation of the radiative effect of haze on the urban hydrological cycle using reanalysis data in beijing. Atmospheric Chemistry and Physics, 19, 7001-7017. doi:doi: 10.5194/acp-19-7001-2019.

Kotroni, V., \& Lagouvardos, K. (1993). Low-level jet streams associated with atmospheric cold fronts: Seven case studies from the fronts 87 experiment. Geophysical Research Letters, 20, 1371-1374. doi:doi: 10.1029/93GL01701.

Kotthaus, S., \& Grimmond, C. (2014a). Energy exchange in a dense urban environment - Part I: Temporal variability of long-term observations in central London. Urban Climate, 10, 261-280. doi:doi: 10.1016/j.uclim.2013.10.002.

Kotthaus, S., \& Grimmond, C. (2014b). Energy exchange in a dense urban environment - Part II: Impact of spatial heterogeneity of the surface. Urban Climate, 10, 281-307. doi:doi: 10.1016/j.uclim.2013.10.001.

Kotthaus, S., \& Grimmond, C. S. B. (2018). Atmospheric Boundary Layer Characteristics from Ceilometer Measurements Part 1: A new method to track mixed layer height and classify clouds. Quarterly Journal of the Royal Meteorological Society, 144, 1525-1538. doi:doi: 10.1002/qj.3299.

Kotthaus, S., Halios, C., Barlow, F. J., \& Grimmond, C. (2018). Volume for pollution dispersion: London's atmospheric boundary layer during clearflo observed with two ground-based lidar types. Atmospheric Environment, 190, 401 - 414. doi:doi: https://doi.org/10.1016/j.atmosenv.2018.06.042. 
Krayenhoff, E. S., Jiang, T., Christen, A., Martilli, A., Oke, T. R., Bailey, B. N., Nazarian, N., Voogt, J. A., Giometto, M. G., Stastny, A., \& Crawford, B. R. (2020). A multi-layer urban canopy meteorological model with trees (bep-tree): Street tree impacts on pedestrian-level climate. Urban Climate, 32, 100590. doi:doi: https://doi.org/10.1016/j.uclim.2020.100590.

Kurppa, M., Hellsten, A., Roldin, P., Kokkola, H., Tonttila, J., Auvinen, M., Kent, C., Kumar, P., Maronga, B., \& Järvi, L. (2019). Implementation of the sectional aerosol module salsa2.0 into the palm model system 6.0: model development and first evaluation. Geoscientific Model Development, 12, 1403-1422. doi:doi: 10.5194/gmd12-1403-2019.

Kusaka, H., Kondo, H., Kikegawa, Y., \& Kimura, F. (2001). A simple single-layer urban canopy model for atmospheric models: Comparison with multi-layer and slab models. Boundary-Layer Meteorology, 101, 329-358. doi:doi: 10.1023/A:1019207923078.

Laaidi, K., Zeghnoun, A., Dousset, B., Vandentorren, S., Giraudet, E., \& Beaudeau, P. (2012). The impact of heat islands on mortality in paris during the august 2003 heat wave. Environmental Health Perspectives, 120, 254-259. doi:doi: 10.1289/ehp.1103532.

Lean, H. W., Barlow, J. F., \& Halios, C. H. (2019). The impact of spin-up and resolution on the representation of a clear convective boundary layer over london in order $100 \mathrm{~m}$ grid-length versions of the met office unified model. Quarterly Journal of the Royal Meteorological Society, 145, 1674-1689. doi:doi: 10.1002/qj.3519.

Lemonsu, A., Bastin, S., Masson, V., \& Drobinski, P. (2006). Vertical structure of the urban boundary layer over marseille under sea-breeze conditions. Boundary-Layer Meteorology, 118, 477-501. doi:doi: 10.1007/s10546-005-7772-y.

Lemonsu, A., Belair, S., \& Mailhot, J. (2009). The New Canadian Urban Modelling System: Evaluation for Two Cases from the Joint Urban 2003 Oklahoma City Experiment. Boundary-Layer Meteorology, 133, 47-79. doi:doi: 10.1007/s10546-009-9414-2.

Levy, R. C., Mattoo, S., Munchak, L. A., Remer, L. A., Sayer, A. M., Patadia, F., \& Hsu, N. C. (2013). The collection 6 modis aerosol products over land and ocean. Atmospheric Measurement Techniques, 6, 2989-3034. doi:doi: 10.5194/amt-6-2989-2013.

Lindberg, F., Grimmond, C., Yogeswaran, N., Kotthaus, S., \& Allen, L. (2013). Impact of city changes and weather on anthropogenic heat flux in europe 1995-2015. Urban Climate, 4, 1 - 15. doi:doi: https://doi.org/10.1016/j.uclim.2013.03.002.

Lock, A., Brown, A., Bush, M., Martin, G., \& Smith, R. (2000). A new boundary layer mixing scheme. part i: Scheme description and single-column model tests. Monthly weather review, 128, 3187-3199.

Loridan, T., \& Grimmond, C. (2012b). Multi-site evaluation of an urban land-surface model: intra-urban heterogeneity, seasonality and parameter complexity requirements. Quarterly Journal of the Royal Meteorological Society, 138, 1094-1113. doi:doi: 
10.1002/qj.963.

Loridan, T., \& Grimmond, C. S. B. (2012a). Characterization of Energy Flux Partitioning in Urban Environments: Links with Surface Seasonal Properties. Journal of Applied Meteorology and Climatology, 51, 219-241. doi:doi: 10.1175/JAMC-D-11-038.1.

Loridan, T., Grimmond, C. S. B., Grossman-Clarke, S., Chen, F., Tewari, M., Manning, K., Martilli, A., Kusaka, H., \& Best, M. (2010). Trade-offs and responsiveness of the single-layer urban canopy parametrization in WRF: An offline evaluation using the MOSCEM optimization algorithm and field observations. Quarterly Journal of the Royal Meteorological Society, 136, 997-1019. doi:doi: 10.1002/qj.614.

Loridan, T., Lindberg, F., Jorba, O., Kotthaus, S., Grossman-Clarke, S., \& Grimmond, C. (2013). High Resolution Simulation of the Variability of Surface Energy Balance Fluxes Across Central London with Urban Zones for Energy Partitioning. BoundaryLayer Meteorology, 147, 493-523. doi:doi: 10.1007/s10546-013-9797-y.

Lundquist, J., \& Mirocha, J. (2008). Interaction of nocturnal low-level jets with urban geometries as seen in joint urban 2003 data. Journal of Applied Meteorology and Climatology, 47, 44-58. doi:doi: 10.1175/2007JAMC1581.1.

Mahrt, L. (1998). Stratified atmospheric boundary layers and breakdown of models. Theoretical and Computational Fluid Dynamics, 11, 263-279. doi:doi: $10.1007 / \mathrm{s} 001620050093$.

Manninen, A. J., O'Connor, E. J., Vakkari, V., \& Petäjä, T. (2016). A generalised background correction algorithm for a halo doppler lidar and its application to data from finland. Atmospheric Measurement Techniques, 9, 817-827.

Maronga, B. et al. (2020). Overview of the palm model system 6.0. Geoscientific Model Development, 13, 1335-1372. doi:doi: 10.5194/gmd-13-1335-2020.

Maronga, B., Gryschka, M., Heinze, R., Hoffmann, F., Kanani-Sühring, F., Keck, M., Ketelsen, K., Letzel, M. O., Sühring, M., \& Raasch, S. (2015). The parallelized large-eddy simulation model (palm) version 4.0 for atmospheric and oceanic flows: model formulation, recent developments, and future perspectives. Geoscientific Model Development, 8, 2515-2551. doi:doi: 10.5194/gmd-8-2515-2015.

Maronga, B., \& Reuder, J. (2017). On the Formulation and Universality of Monin-Obukhov Similarity Functions for Mean Gradients and Standard Deviations in the Unstable Surface Layer: Results from Surface-Layer-Resolving Large-Eddy Simulations. Journal of the Atmospheric Sciences, 74, 989-1010. doi:doi: 10.1175/JAS-D16-0186.1.

Martilli, A., Clappier, A., \& Rotach, M. W. (2002). An Urban Surface Exchange Parameterisation for Mesoscale Models. Boundary-Layer Meteorology, 104, 261-304. doi:doi: 10.1023/A:1016099921195. 
Masson, V. (2000). A Physically-Based Scheme For The Urban Energy Budget In Atmospheric Models. Boundary-Layer Meteorology, 94, 357-397. doi:doi: 10.1023/A:1002463829265.

Masson, V., Lemonsu, A., Hidalgo, J., \& Voogt, J. (2020). Urban climates and climate change. Annual Review of Environment and Resources, 45, 411-444. doi:doi: 10.1146/annurev-environ-012320-083623.

Miao, S., Chen, F., LeMone, M. A., Tewari, M., Li, Q., \& Wang, Y. (2009). An Observational and Modeling Study of Characteristics of Urban Heat Island and Boundary Layer Structures in Beijing. Journal of Applied Meteorology and Climatology, 48, 484-501. doi:doi: 10.1175/2008JAMC1909.1.

Miao, Y., Liu, S., Sheng, L., Huang, S., \& Li, J. (2019). Influence of boundary layer structure and low-level jet on $\mathrm{pm}(2.5)$ pollution in beijing: A case study. International journal of environmental research and public health, 16, 616. doi:doi: 10.3390/ijerph16040616.

Mikio, N., \& Hiroshi, N. (2009). Development of an improved turbulence closure model for the atmospheric boundary layer. Journal of the Meteorological Society of Japan. Ser. II , 87, 895-912. doi:doi: 10.2151/jmsj.87.895.

Mitchell, J. (1961). The temperature of cities. Weatherwise, 14, 224-229.

Moeng, C.-H., \& Wyngaard, J. C. (1988). Spectral Analysis of Large-Eddy Simulations of the Convective Boundary Layer. Journal of the Atmospheric Sciences, 45, 3573-3587. doi:doi: 10.1175/1520-0469(1988)045;3573:SAOLES ¿2.0.CO;2.

Morris, C., \& Simmonds, I. (2001). Quantification of the influence of wind and cloud on the nocturnal urban heat island of a large city. Journal of Applied Meteorology, 40, 169-182. doi:doi: 10.1175/1520-0450(2001)040;0169:QOTIOW ¿2.0.CO;2.

NASA/EOSDIS (2019). NASA Worldview.

Nemunaitis-Berry, K. L., Klein, P. M., Basara, J. B., \& Fedorovich, E. (2017). Sensitivity of Predictions of the Urban Surface Energy Balance and Heat Island to Variations of Urban Canopy Parameters in Simulations with the WRF Model. Journal of Applied Meteorology and Climatology, 56, 573-595. doi:doi: 10.1175/JAMC-D-16-0157.1.

NOAA/NCDC (2012). Climate Data Online.

Oikonomou, E., Davies, M., Mavrogianni, A., Biddulph, P., Wilkinson, P., \& Kolokotroni, M. (2012). Modelling the relative importance of the urban heat island and the thermal quality of dwellings for overheating in London. Building and Environment, 57, 223 238. doi:doi: https://doi.org/10.1016/j.buildenv.2012.04.002.

Oke, T. (1973). City size and the urban heat island. Atmospheric Environment (1967), 7, 769 - 779. doi:doi: https://doi.org/10.1016/0004-6981(73)90140-6.

Oke, T. (1987). Boundary Layer Climates (2nd ed.). London and John Wiley \& Sons, 
New York (1987) Co-published by Routledge.

Oke, T., \& East, C. (1971). The urban boundary layer in montreal. Boundary-Layer Meteorology, 1, 411-437. doi:doi: 10.1007/BF00184781.

Olson, J. B., Kenyon, J. S., Angevine, W. M., Brown, J. M., Pagowski, M., \& Sušelj, K. (2019). A description of the mynn-edmf scheme and the coupling to other components in wrf-arw. noaa technical memorandum oar gsd, 61, pp. 37. doi:doi: 10.25923/n9wmbe49.

Oreopoulos, L., \& Barker, H. W. (1999). Accounting for subgrid-scale cloud variability in a multi-layer 1d solar radiative transfer algorithm. Quarterly Journal of the Royal Meteorological Society, 125, 301-330. doi:doi: 10.1002/qj.49712555316.

Pal, S. et al. (2012). Spatio-temporal variability of the atmospheric boundary layer depth over the paris agglomeration: An assessment of the impact of the urban heat island intensity. Atmospheric Environment, 63, 261 - 275. doi:doi: https://doi.org/10.1016/j.atmosenv.2012.09.046.

Parish, T. R. (2017). On the Forcing of the Summertime Great Plains Low-Level Jet. Journal of the Atmospheric Sciences, 74, 3937-3953. doi:doi: 10.1175/JAS-D-17-0059.1.

Park, J., Basu, S., \& Manuel, L. (2014). Large-eddy simulation of stable boundary layer turbulence and estimation of associated wind turbineâ $€ \%$ oloads. Wind Energy, 17, 359-384. doi:doi: 10.1002/we.1580.

Pesaresi, M., Melchiorri, M., Siragusa, A., \& Kemper, T. (2016). Atlas of the human planet-mapping human presence on earth with the global human settlement layer. jrc103150. Publications Office of the European Union. Luxembourg (Luxembourg): European Commission, doi:doi: JRC103150.

Piacsek, S. A., \& Williams, G. P. (1970). Conservation properties of convection difference schemes. Journal of Computational Physics, 6, 392 - 405. doi:doi: https://doi.org/10.1016/0021-9991(70)90038-0.

Pichugina, Y. L., \& Banta, R. M. (2010). Stable Boundary Layer Depth from HighResolution Measurements of the Mean Wind Profile. Journal of Applied Meteorology and Climatology, 49, 20-35. doi:doi: 10.1175/2009JAMC2168.1.

Pigeon, G., Lemonsu, A., Grimmond, C., Durand, P., Thouron, O., \& Masson, V. (2007). Divergence of turbulent fluxes in the surface layer: Case of a coastal city. BoundaryLayer Meteorology, 124, 269-290.

Porson, A., Clark, P. A., Harman, I., Best, M., \& Belcher, S. (2010). Implementation of a new urban energy budget scheme in the metum. part i: Description and idealized simulations. Quarterly Journal of the Royal Meteorological Society, 136, 1514-1529.

Powers, J. G. et al. (2017). The weather research and forecasting model: Overview, system efforts, and future directions. Bulletin of the American Meteorological Society, 
98, 1717-1737. doi:doi: 10.1175/BAMS-D-15-00308.1.

Redon, E., Lemonsu, A., \& Masson, V. (2020). An urban trees parameterization for modeling microclimatic variables and thermal comfort conditions at street level with the town energy balance model (teb-surfex v8.0). Geoscientific Model Development, 13, 385-399. doi:doi: 10.5194/gmd-13-385-2020.

Resler, J., Krč, P., Belda, M., Juruš, P., Benešová, N., Lopata, J., Vlček, O., Damašková, D., Eben, K., Derbek, P., Maronga, B., \& Kanani-Sühring, F. (2017). Palm-usm v1.0: A new urban surface model integrated into the palm large-eddy simulation model. Geoscientific Model Development, 10, 3635-3659. doi:doi: 10.5194/gmd-10-3635-2017.

Ronda, R. J., Steeneveld, G. J., Heusinkveld, B. G., Attema, J. J., \& Holtslag, A. A. M. (2017). Urban finescale forecasting reveals weather conditions with unprecedented detail. Bulletin of the American Meteorological Society, 98, 2675-2688. doi:doi: 10.1175/BAMS-D-16-0297.1.

Roth, M. (2000). Review of atmospheric turbulence over cities. Quarterly Journal of the Royal Meteorological Society, 126, 941-990. doi:doi: 10.1002/qj.49712656409.

Salamanca, F., \& Martilli, A. (2009). A new Building Energy Model coupled with an Urban Canopy Parameterization for urban climate simulations-part II. Validation with one dimension off-line simulations. Theoretical and Applied Climatology, 99, 345. doi:doi: 10.1007/s00704-009-0143-8.

Schmid, H. P., Cleugh, H. A., Grimmond, C. S. B., \& Oke, T. R. (1991). Spatial variability of energy fluxes in suburban terrain. Boundary-Layer Meteorology, 54, 249-276. doi:doi: 10.1007/BF00183956.

Shapiro, A., \& Fedorovich, E. (2008). Coriolis effects in homogeneous and inhomogeneous katabatic flows. Quarterly Journal of the Royal Meteorological Society, 134, 353-370. doi:doi: 10.1002/qj.217.

Shapiro, A., Fedorovich, E., \& Rahimi, S. (2016). A unified theory for the great plains nocturnal low-level jet. Journal of the Atmospheric Sciences, 73, 3037-3057. doi:doi: 10.1175/JAS-D-15-0307.1.

Shepherd, J. M. (2009). A Review of Current Investigations of Urban-Induced Rainfall and Recommendations for the Future. Earth Interactions, 9, 1-27. doi:doi: 10.1175/EI156.1.

Skamarock, W. C., Klemp, J. B., Dudhia, J., Gill, D. O., Barker, D. M., Wang, W., \& Powers, J. G. (2008). A description of the Advanced Research WRF version 3. NCAR Technical note $-475+$ STR.

Skamarock, W. C., Klemp, J. B., Dudhia, J., Gill, D. O., Liu, Z., Berner, J., Wang, W., Powers, J. G., Duda, M. G., Barker, D. M., \& Huang, X.-Y. (2019). A: A Description of the Advanced Research WRF Version 4. NCAR Tech. Note NCAR/TN-556+STR, 
145 pp. doi:doi: 10.5065/1dfh-6p97.

Smith, R. (1990). A scheme for predicting layer clouds and their water content in a general circulation model. Quarterly Journal of the Royal Meteorological Society, 116, $435-460$.

Song, J., \& Wang, Z.-H. (2015). Interfacing the urban land-atmosphere system through coupled urban canopy and atmospheric models. Boundary-Layer Meteorology, 154, 427-448. doi:doi: 10.1007/s10546-014-9980-9.

Song, J., \& Wang, Z.-H. (2016). Evaluating the impact of built environment characteristics on urban boundary layer dynamics using an advanced stochastic approach. Atmospheric Chemistry and Physics, 16, 6285-6301. doi:doi: 10.5194/acp-16-6285-2016.

Steeneveld, G.-J. (2014). Current challenges in understanding and forecasting stable boundary layers over land and ice. Frontiers in Environmental Science, 2, 41. doi:doi: 10.3389/fenvs.2014.00041.

Steeneveld, G.-J. et al. (2017). Single-column urban boundary layer inter-comparison modelling experiment (SUBLIME): call for participation. Urban Climate News, 66, $21-26$.

Sterk, H. A. M., Steeneveld, G. J., \& Holtslag, A. A. M. (2013). The role of snow-surface coupling, radiation, and turbulent mixing in modeling a stable boundary layer over arctic sea ice. Journal of Geophysical Research: Atmospheres, 118, 1199-1217. doi:doi: $10.1002 /$ jgrd.50158.

Stewart, I. D., \& Oke, T. R. (2012). Local Climate Zones for Urban Temperature Studies. Bulletin of the American Meteorological Society, 93, 1879-1900. doi:doi: 10.1175/BAMS-D-11-00019.1.

Storm, B., Dudhia, J., Basu, S., Swift, A., \& Giammanco, I. (2009). Evaluation of the weather research and forecasting model on forecasting low-level jets: implications for wind energy. Wind Energy, 12, 81-90. doi:doi: 10.1002/we.288.

Sukoriansky, S., Galperin, B., \& Perov, V. (2005). Application of a new spectral theory of stably stratified turbulence to the atmospheric boundary layer over sea ice. BoundaryLayer Meteorology, 117, 231-257. doi:doi: 10.1007/s10546-004-6848-4.

Theeuwes, N. E., Barlow, J. F., Teuling, A. J., Grimmond, C. S. B., \& Kotthaus, S. (2019). Persistent cloud cover over mega-cities linked to surface heat release. npj Climate and Atmospheric Science, 2, 1-6.

Theeuwes, N. E., Steeneveld, G.-J., Ronda, R. J., Rotach, M. W., \& Holtslag, A. A. M. (2015). Cool city mornings by urban heat. Environmental Research Letters, 10, 114022. doi:doi: 10.1088/1748-9326/10/11/114022.

Thompson, G., Field, P. R., Rasmussen, R. M., \& Hall, W. D. (2008). Explicit forecasts of winter precipitation using an improved bulk microphysics scheme. part ii: Imple- 
mentation of a new snow parameterization. Monthly Weather Review, 136, 5095-5115. doi:doi: 10.1175/2008MWR2387.1.

Tsiringakis, A., Holtslag, A. A. M., Grimmond, S., \& Steeneveld, G. J. (2020). Surface and atmospheric driven variability of the single-layer urban canopy model under clear-sky conditions over london. Journal of Geophysical Research: Atmospheres, 125, e2019JD032167. doi:doi: https://doi.org/10.1029/2019JD032167.

Tsiringakis, A., Steeneveld, G., Holtslag, A. M., Albert, Kotthaus, S., \& Grimmond, C. S. B. (2019). On- and off-line evaluation of the single-layer urban canopy model in london summertime conditions. Quarterly Journal of the Royal Meteorological Society, 145, 1474-1489. doi:doi: 10.1002/qj.3505.

UN (2014). World urbanization prospects: The 2014 revision. Renewable EnergyUnited Nations Department of Economic and Social Affairs Population Division, .

Uno, I., Wakamatsu, S., H., U., \& Nakamura, A. (1992). Observed structure of the nocturnal urban boundary layer and its evolution into a convective mixed layer. Atmospheric Environment. Part B. Urban Atmosphere, 26, 45 - 57. doi:doi: https://doi.org/10.1016/0957-1272(92)90036-R.

Uno, I., Wakamatsu, S., Ueda, H., \& Nakamura, A. (1988). An observational study of the structure of the nocturnal urban boundary layer. Boundary-Layer Meteorology, 45, 59-82. doi:doi: 10.1007/BF00120815.

UWYO (2012). Atmospheric soundings.

Vakkari, V., Manninen, A. J., O'Connor, E. J., Schween, J. H., van Zyl, P. G., \& Marinou, E. (2019). A novel post-processing algorithm for halo doppler lidars. Atmospheric Measurement Techniques (AMT), 12, 839-852.

Vanderwende, B., Lundquist, J., Rhodes, M., Takle, E., \& Irvin, S. (2015). Observing and simulating the summertime low-level jet in central iowa. Monthly Weather Review, 143, 2319-2336. doi:doi: 10.1175/MWR-D-14-00325.1.

Varentsov, M., Wouters, H., Platonov, V., \& Konstantinov, P. (2018). Megacity-induced mesoclimatic effects in the lower atmosphere: A modeling study for multiple summers over moscow, russia. Atmosphere, 9. doi:doi: 10.3390/atmos9020050.

Walsh, J. E., Chapman, W. L., Romanovsky, V., Christensen, J. H., \& Stendel, M. (2008). Global Climate Model Performance over Alaska and Greenland. Journal of Climate, 21, 6156-6174. doi:doi: 10.1175/2008JCLI2163.1.

Wang, W. (2009). The influence of thermally-induced mesoscale circulations on turbulence statistics over an idealized urban area under a zero background wind. Boundary-Layer Meteorology, 131, 403-423. doi:doi: 10.1007/s10546-009-9378-2.

Wang, Y., Klipp, C., Garvey, D., Ligon, D., Williamson, C., Chang, S., Newsom, R., \& Calhoun, R. (2007). Nocturnal low-level-jet-dominated atmospheric boundary layer 
observed by a doppler lidar over oklahoma city during ju2003. Journal of Applied Meteorology and Climatology, 46, 2098-2109. doi:doi: 10.1175/2006JAMC1283.1.

Wang, Z.-H., Bou-Zeid, E., Au, S., \& Smith, J. (2011). Analyzing the sensitivity of WRF's single-layer urban canopy model to parameter uncertainty using advanced Monte Carlo simulation. Journal of Applied Meteorology and Climatology, 50, 1795-1814. doi:doi: 10.1175/2011JAMC2685.1.

Ward, H., Kotthaus, S., Järvi, L., \& Grimmond, C. (2016). Surface urban energy and water balance scheme (suews): Development and evaluation at two uk sites. Urban Climate, 18, 1 - 32. doi:doi: https://doi.org/10.1016/j.uclim.2016.05.001.

Van de Wiel, B. J. H., Moene, A. F., Steeneveld, G. J., Baas, P., Bosveld, F. C., \& Holtslag, A. A. M. (2010). A Conceptual View on Inertial Oscillations and Nocturnal Low-Level Jets. Journal of the Atmospheric Sciences, 67, 2679-2689. doi:doi: 10.1175/2010JAS3289.1.

de Wiel, B. J. H. V., Moene, A. F., \& Jonker, H. J. J. (2012). The cessation of continuous turbulence as precursor of the very stable nocturnal boundary layer. Journal of the Atmospheric Sciences, 69, 3097 - 3115. doi:doi: 10.1175/JAS-D-12-064.1.

Wieringa, J. (1993). Representative roughness parameters for homogeneous terrain. Boundary-Layer Meteorology, 63, 323-363. doi:doi: 10.1007/BF00705357.

Wood, C. R., Lacser, A., Barlow, J. F., Padhra, A., Belcher, S. E., Nemitz, E., Helfter, C., Famulari, D., \& Grimmond, C. S. B. (2010). Turbulent flow at $190 \mathrm{~m}$ height above london during 2006-2008: A climatology and the applicability of similarity theory. Boundary-Layer Meteorology, 137, 77 - 96. doi:doi: 10.1007/s10546-010-9516-x.

Yang, J., Wang, Z.-H., Chen, F., Miao, S., Tewari, M., Voogt, J. A., \& Myint, S. (2015). Enhancing Hydrologic Modelling in the Coupled Weather Research and Forecasting-Urban Modelling System. Boundary-Layer Meteorology, 155, 87-109. doi:doi: 10.1007/s10546-014-9991-6.

Yang, J., Wang, Z.-H., Georgescu, M., Chen, F., \& Tewari, M. (2016). Assessing the impact of enhanced hydrological processes on urban hydrometeorology with application to two cities in contrasting climates. Journal of Hydrometeorology, 17, 1031-1047. doi:doi: 10.1175/JHM-D-15-0112.1.

Yoshikado, H. (1992). Numerical study of the daytime urban effect and its interaction with the sea breeze. Journal of Applied Meteorology, 31, 1146-1164. doi:doi: 10.1175/15200450(1992)031¡1146:nsotdui2.0.co;2.

Zhang, C., Wang, Y., \& Hamilton, K. (2011). Improved representation of boundary layer clouds over the southeast pacific in arw-wrf using a modified tiedtke cumulus parameterization scheme. Monthly Weather Review, 139, 3489-3513. doi:doi: 10.1175/MWRD-10-05091.1. 
Zhao, W., Zhang, N., Sun, J., \& Zou, J. (2014). Evaluation and Parameter-Sensitivity Study of a Single-Layer Urban Canopy Model (SLUCM) with Measurements in Nanjing China. Journal of Hydrometeorology, 15, 1078-1090. doi:doi: 10.1175/JHM-D-130129.1. 



\section{Acknowledgements}

I spent quite some time thinking at what languages should I write this section of the thesis. You would expect that a person living in the Netherlands for six and a half years now should write at least one part of the acknowledgements in Dutch, or should I say in Nederlands to be precise. But what if that person is Greek? Then at least one section

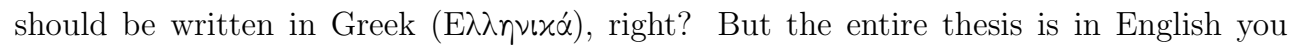
would say! Then I will write it using all three of them, to a certain degree of course.

The last 4 and half years have been a long journey into science, research and discovery. This journey I did not undertake alone as rarely any person ever does. Friends, colleagues, mentors, family all contributed to the collection of amazing and memorable experiences that constitute my life during the $\mathrm{PhD}$. As is the custom, this section is dedicated to all of you! I tried to remember everyone who has been with me or helped me in this journey, but alas some I will forget at the moment of writing. For that I am really sorry! Yet, if you do indeed find yourselves a bit left out for not being mentioned here, please remember that the memories you have with me are real and worth remembering.

Gert-Jan, you have been a constant source of guidance and motivation during this journey! I still vividly remember the first time we met to discuss about me doing my master thesis on gravity-wave drag. That's where I think this story truly began. I can not thank you enough for all your help and support during the years that we worked together. You were always there to listen to my questions, all the new things I discovered and wanted to discuss, even if I was knocking at your office door at 18:30 (while you were getting ready to go back home). If you hadn't motivated me to convert my research findings into something meaningful and easy for others to understand, this thesis would have been even more gibberish than it already is. Je bent niet alleen een geweldige begeleider geweest, maar ook een echte mentor voor mij! Heel erg bedankt daarvoor!

Bert I want to thank you very much for being an awesome promotor during my PhD. Your positive point of view always helped me to see the good things in my research! I will not forget our discussion on boundary-layer theory. Thank you for literally being a promotor of my research during your trips to the BLM conferences in the USA. It has been an honour to work with you. Jordi thank you for all the amazing discussions and hard inquisitive questions that always motivated me to look on my research/results from 
a different point of view, the helicopter view!

Natalie, it has been a pleasure collaborating with you during my $\mathrm{PhD}$ and I am glad we are again colleagues in KNMI! Thank you for all your help with our work on low-level jets and for inviting me to visit the department of Meteorology in Reading University. Sue, Janet and Simone, it has been an honour to collaborate with you during my PhD! I really thank you for all the time you devoted into reading my work. Your sharp comments and insights on my research have made me a better researcher.

Bert and Oscar, it has been a pleasure and privilege to work in the field with you. You have taught me a lot about the observational techniques and helped me to understand how challenging is to plan, prepare and conduct observations in the urban environment. Of course I cannot forget Kees and Reinder. Without your help, I would have still been trying to set-up WRF and PALM on the Wageningen HPC and Cartesius systems. Chiel and Maarten thank you for your always sharp questions and discussions during and after T-talks! Laurens, Wouter, Folkert, Arnold and Ingrid thank you for all the wonderful discussion during coffee and lunch breaks.

To my PhD office mates and friends a big thank you for the wonderful moments during these 4 years! Auke, you have been a great friend and scientific debate partner all these years, with a keen eye on always improving my dreadfully looking figures. Arjan for all our amazing discussions during coffee breaks, accompanied by your superb desserts. Sjoerd for the active participation (and organisation) in the cocktail afternoons (and the delicious gnocchi your brought at the PhD dinners) and Ruben for the scientific discussions without end. We surely prepared together the greatest pub quiz BBOS has even seen!

To my PhD mates across the corridor, my time in the department would not have been the same without you! Thank you Xavi for Biertjeklaas and your comments of campus food quality, Imme for ruining every spontaneous joke we tried to make, Alba for the amazing packages of food from Spain, Stijn for your incredible party mood, Anja for our nice discussion on gaming and Martin for you always positive attitude! Also many thanks to Naomi for the amazing vegan cakes and game nights and Liesbeth for carrying the MAQ football team to the finals!

As you all know, life requires balance. For everyone good moment at work an equally good moment in personal life must exist to make a person happy. For all these personal moments I want to deeply my family and friends! Both my most recent (Menia, Kassiani and Marion) and older (Pablo, Raquel, Fotini) friends and housemates. Without you and all the amazing dinners, parties, movies, gatherings and chill moment we spend together during these 4 years life would not have been the same. To my best friends from Greece: Thanasis, Spiros, Christos, Sofia, Giannis, Nikos, John and from abroad: Andreas and Duick! You are amazing friends, some of you I know for more than a decade. I cherish the wonderful moments we spent together (online and in person) during my time in the Netherlands! I certainly look forward to even more memorable dinners, trips, parties and 
conversations with all of you, when we finally escape corona's grip!

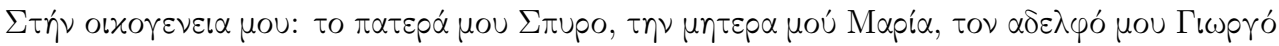

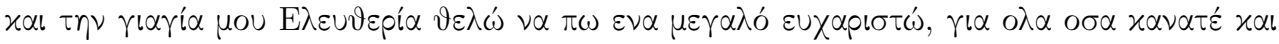

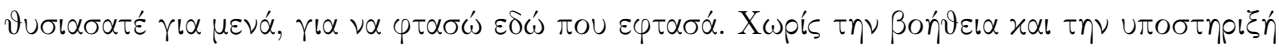

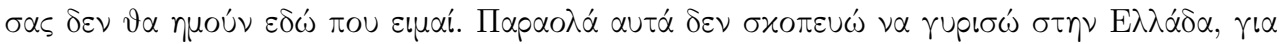

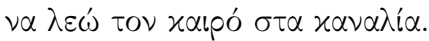

Last but not least, I want to thank the person who has been with me during this journey for the last 4 years. You have seen grumpy, happy, sad, stressed and yet you always supported me during my most difficult and happy moments of this journey. Thank you Sara!

Finally, I want to express my gratitude to Wageningen University, the graduate schools and organisations that make this campus a place where science, knowledge and communication can flourish. To some of you this opinion might come as a surprise, considering I have been vocal about the dreadful food on campus and criticised university decisions more than once. Yet, every now and then it is crucial to acknowledge and cherish the things we take for granted, when living in such a privileged environment. In the life of a scientist acknowledgement and criticism often go hand in hand and since my criticism is often known I thought it was appropriate to close this thesis with an acknowledgement! 



\section{List of Publications}

1. Tsiringakis, A., Steeneveld, G.J. and Holtslag, A.A.M. (2017), Small-scale orographic gravity wave drag in stable boundary layers and its impact on synoptic systems and near-surface meteorology. Q.J.R. Meteorol. Soc., 143: 1504-1516. https://doi.org/10.1002/qj.3021

2. Tsiringakis, A, Steeneveld, G-J, Holtslag, AAM, Kotthaus, S, Grimmond, S. (2019). On- and off-line evaluation of the single-layer urban canopy model in London summertime conditions. Q J R Meteorol Soc. 2019; 145: 1474- 1489. https://doi.org/10.1002/qj.3505

3. Tsiringakis, A., Holtslag, A. A. M., Grimmond, S. and Steeneveld, G. J. (2020). Surface and atmospheric driven variability of the single-layer urban canopy model under clear-sky conditions over London. Journal of Geophysical Research: Atmospheres, 125, e2019JD032167. https://doi.org/10.1029/2019JD032167

4. Tsiringakis, A., Theeuwes, N., Barlow, J. and Steeneveld, G. J. (2021). Interactions Between the Nocturnal Low-Level Jets and the Urban Boundary Layer: a Case Study over London. under review.

5. Steeneveld, G.J., Tsiringakis, A., Masson, V., Kotthaus, S., Grimmond, S., Bohnenstengel, S., Halios, C., van den Oord, G., Schubert, S., Stöckl, S. and Young. D. (2021): Single-column Urban Boundary Layer Inter-comparison Modelling Experiment (SUBLIME) part A: model comparison set up and case study, in preparation

6. Steeneveld, G.J., Tsiringakis, A., Masson, V., Barlow, J., Bohnenstengel, S., Halios, C., Grimmond, S., van Haren, R., Kotthaus, S., Lee, S.H., Lipson, M., Lobocki, L., McNorton, J., van den Oord, G., Schubert, S., Song, J., Stockl, S., Wouters, H., Demuzere, M. and Young, D. (2021): Single-column Urban Boundary Layer Inter-comparison Modelling Experiment (SUBLIME) part B: results. In preparation. 


\section{SENSE}

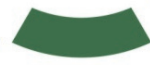

Netherlands Research School for the

Socio-Economic and Natural Sciences of the Environment

\section{I P L O M A}

for specialised PhD training

The Netherlands research school for the Socio-Economic and Natural Sciences of the Environment (SENSE) declares that

\section{Aristofanis Tsiringakis}

born on 17 May 1991 in Kerkyra, Greece

has successfully fulfilled all requirements of the educational PhD programme of SENSE.

Wageningen, 21 April 2021
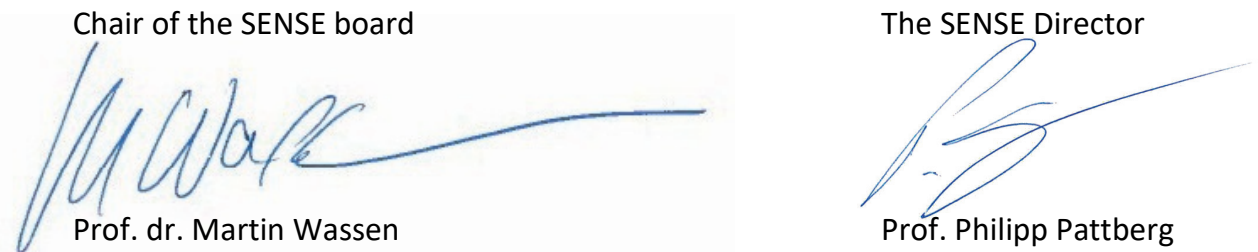

The SENSE Research School has been accredited by the Royal Netherlands Academy of Arts and Sciences (KNAW)

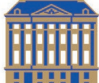

$\begin{array}{lllllllllllllllllllllll}K & O & N & I & N & K & L & I & J & K & E & N & E & D & E & R & L & A & N & D & S & E\end{array}$ 


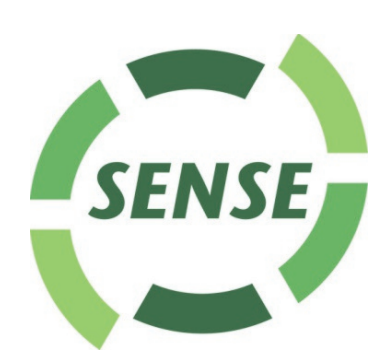

The SENSE Research School declares that Aristofanis Tsiringakis has successfully fulfilled all requirements of the educational $\mathrm{PhD}$ programme of SENSE with a work load of 37.7 EC, including the following activities:

\section{$\underline{\text { SENSE PhD Courses }}$}

- Environmental research in context (2017)

- Research in context activity: 'Developing and featuring video log (vlog) on participation in the International Conference on Urban Climate (New York, August 2018)'

\section{Other PhD and Advanced MSc Courses}

- Energy-efficient scalable algorithms for weather prediction at exascale, Niels Bohr Institute, University of Copenhagen and Danish Meteorological Institute (2017)

- Physical parameterization of sub-grid scale processes, European Centre for MediumRange Weather Forecasts (2018)

- Career Orientation, Wageningen Graduate Schools (2019)

\section{Management and Didactic Skills Training}

- Co-organization of ILeaps workshop: Modelling and Observing urban fluxes, Wageningen Universisty and Amsterdam Institute for Advanced Metropolitan Solutions (2019)

o Teaching assistant in the BSc course 'Meteorology and Climate' (2017-2019)

\section{Oral Presentations}

- The impact of urbanization on an extreme precipitation event over Jakarta. EMS Annual Meeting: European Conference for Applied Meteorology and Climatology, 4-8 September 2017, Dublin, Ireland

- Evaluation of the Single-Layer Urban Canopy Model: An analysis of model sensitivity to uncertain urban parameters. 10th International Conference on Urban Climate, 6-10 August 2018, New York, United States of America

- Surface and atmospheric driven sensitivity of the single-layer urban canopy model under clear sky conditions in London. EMS Annual Meeting: European Conference for Applied Meteorology and Climatology, 9-13 September 2019, Lyngby, Denmark

- Meteorology and the City. CITIES4PEOPLE: intelligent data analytics for next-gen cities, 15-16 November 2018, 's-Hertogenbosch, The Netherlands

SENSE coordinator PhD education

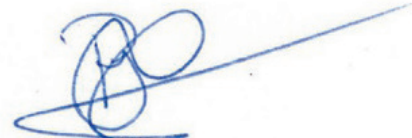

Dr. ir. Peter Vermeulen 
This research received funding from The Netherlands Organisation for Scientific Research (NWO), grant number 864.14.007.

Part of the data analysis and simulations were performed on the supercomputing facilities offered by The Netherlands Organization for Scientific Research (NWO), grant number SH-312-15.

Financial support from Wageningen University for printing this thesis is gratefully acknowledged.

Cover design by Aristofanis Tsiringakis and Sara Issova.

Thesis printed by ProefschriftMaken. 
(1)

(1)

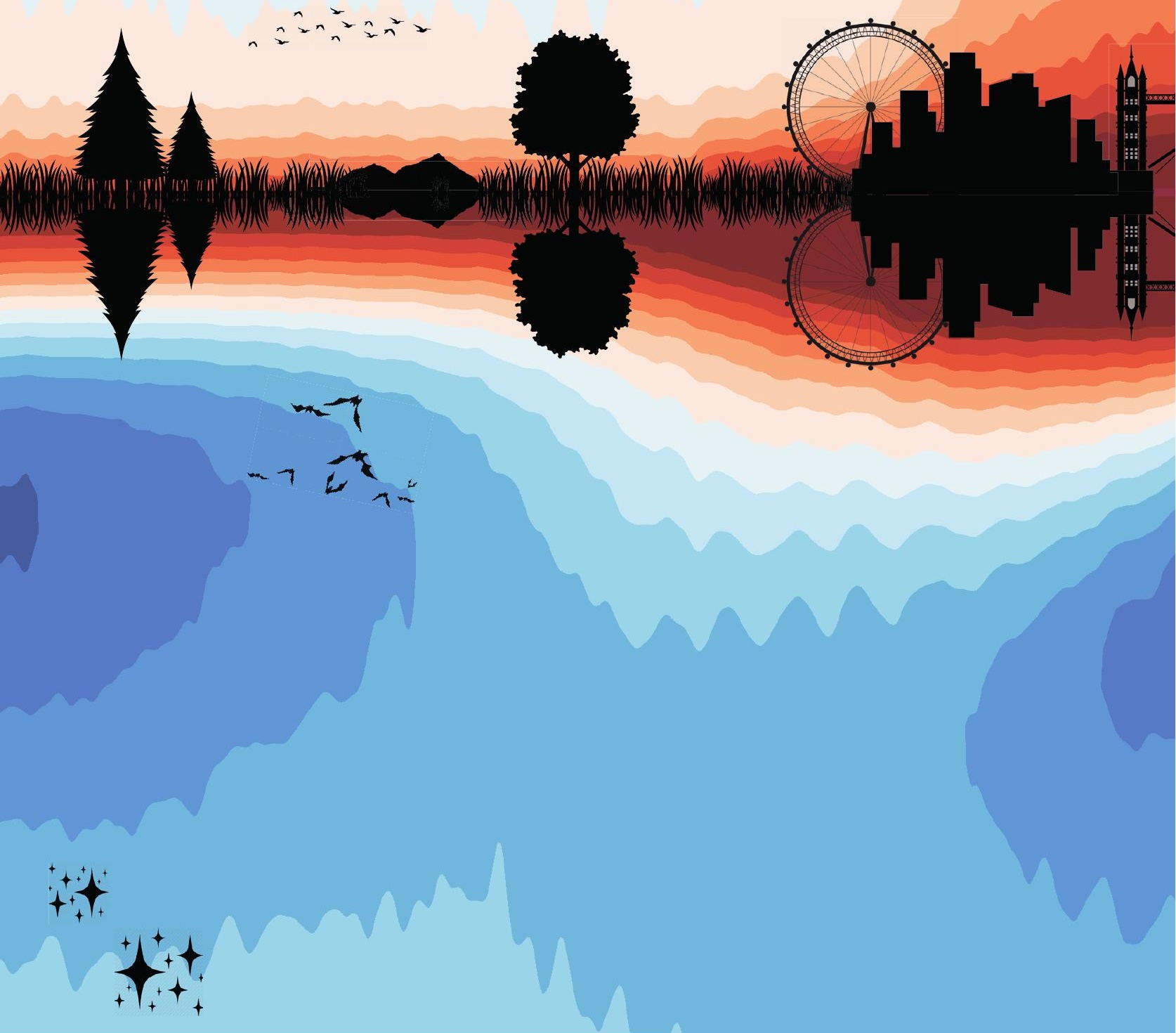

